Florida International University FIU Digital Commons

6-29-2016

\title{
Gold Nanoparticle-Based Colorimetric Sensors for Detection of DNA and Small Molecules
}

Pingping Liang

Florida International University, plian002@fiu.edu

DOI: $10.25148 /$ etd.FIDC000729

Follow this and additional works at: https://digitalcommons.fiu.edu/etd

Part of the Analytical Chemistry Commons

\section{Recommended Citation}

Liang, Pingping, "Gold Nanoparticle-Based Colorimetric Sensors for Detection of DNA and Small Molecules" (2016). FIU Electronic Theses and Dissertations. 2595.

https://digitalcommons.fiu.edu/etd/2595 


\section{FLORIDA INTERNATIONAL UNIVERSITY}

Miami, Florida

GOLD NANOPARTICLE-BASED COLORIMETRIC SENSORS FOR DETECTION OF DNA AND SMALL MOLECULES

A dissertation submitted in partial fulfillment of the requirements for the degree of

DOCTOR OF PHILOSOPHY

in

CHEMISTRY

by

Pingping Liang 
To: Dean Michael R. Heithaus

College of Arts, Sciences and Education

This dissertation, written by Pingping Liang, and entitled Gold Nanoparticle-Based Colorimetric Sensors for Detection of DNA and Small Molecules, having been approved in respect to style and intellectual content, is referred to you for judgment.

We have read this dissertation and recommend that it be approved.

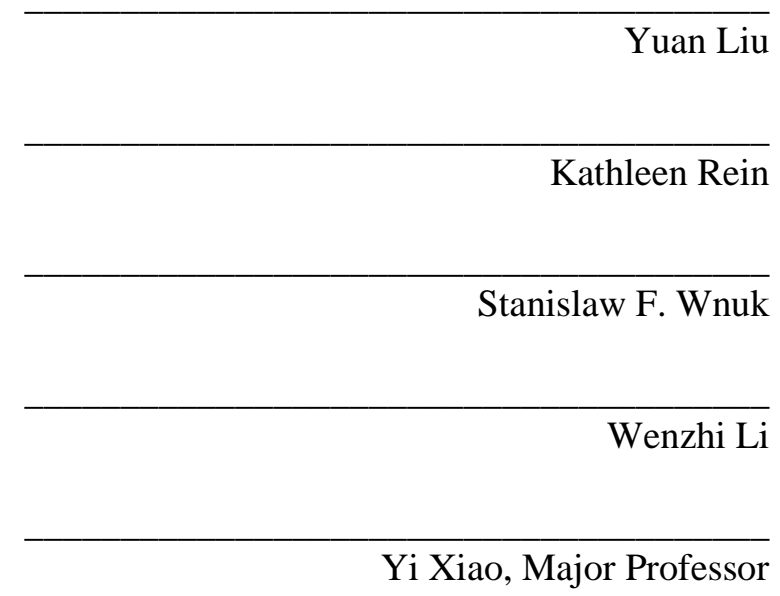

Date of Defense: June 29, 2016

The dissertation of Pingping Liang is approved.

Dean Michael R. Heithaus College of Arts, Sciences and Education

Andrés G. Gil

Vice President for Research and Economic Development and Dean of the University Graduate School

Florida International University, 2016 
C Copyright 2016 by Pingping Liang All rights reserved. 


\section{DEDICATION}

I dedicate this work to my husband Dr. Yong Liang, daughter Grace Liang, parents, sister and brother for their love and support. Without their understanding and encouragement, the completion of this work would not have been possible. 


\section{ACKNOWLEDGMENTS}

The accomplishment of this dissertation would not have been possible without the guidance and the help of many individuals who contributed the countless time and valuable assistance. It is my great pleasure to express my gratitude to all of them in my humble acknowledgment.

First and foremost, I would like to thank my major professor Dr. Yi Xiao for the opportunity to study and work in her group as a Ph.D. graduate student. I would like to express my utmost gratitude for her endless support, supervision, guidance, inspiration and invaluable encouragement which help me to overcome lots of obstructions during the past five years.

I am grateful to all the members of my dissertation committee, Dr. Yuan Liu, Dr. Wenzhi Li, Dr. Kathleen Rein, and Dr. Stanislaw Wnuk for their willingness to serve on my dissertation committee and their valuable time, advice and constant support during the past five years.

I would like to acknowledge Department of Chemistry and Biochemistry and Dissertation Year Fellowship from University Graduate School at Florida International University for the financial support. Big thanks to GPSC, the dean's office of the College of Arts, Sciences and Education for travel funding, without which this Ph.D. would not have been possible. Many thanks go to all the faculty and staff members of the chemistry department for the support, help and coordination. I also would like to thank Dr. Zhihong Nie and his student Zhiqi Huang from University of Maryland for obtaining high-resolution TEM image of gold nanoparticles.

I would like to thank my lab mates for their support and patience. You guys are always there for me. Because of you, my five years are full of great memories. I also would like to thank my husband Yong Liang, my parents, and in-laws for their endless support and love. I am grateful to have my daughter Grace Liang brought into my life.

Finally, I wish to express my appreciation to those who have helped me in any respect during my five years study at FIU, as well as expressing my apology that I could not mention personally one by one. 
ABSTRACT OF THE DISSERTATION

GOLD NANOPARTICLE-BASED COLORIMETRIC SENSORS FOR DETECTION OF DNA

AND SMALL MOLECULES

by

\author{
Pingping Liang \\ Florida International University, 2016 \\ Miami, Florida \\ Professor Yi Xiao, Major Professor
}

Biosensors have proven to be a powerful tool for detecting diverse targets, such as proteins, DNA, and small molecules representing disease biomarkers, toxins, drugs and their metabolites, environmental pollutants, agrichemicals, and antibiotics with high sensitivity and specificity.

The major objective of the research described in this dissertation was to develop low cost, low sample volume, highly sensitive and specific AuNP-based colorimetric sensor platforms for the detection of DNA and small molecules. With this in mind, we propose an instrument-free approach in chapter three for the detection of NADH with a sensor constructed on a paper substrate, based on the target-induced inhibition of AuNP dissolution. The successful detection of this important molecule opens the door to numerous possibilities for dehydrogenase characterization, because $\mathrm{NAD}^{+} / \mathrm{NADH}$ are essential cofactors for more than 300 dehydrogenase enzymes. To further increase the sensitivity of our hybridization-based assay for DNA detection, we developed an enzyme-assisted target recycling (EATR) strategy in chapter four and have applied such an EATR-based colorimetric assay to detect single-nucleotide mismatches in a target DNA with DNA-functionalized AuNPs. This assay is based on the principle that nuclease enzymes recognize probe-target complexes, cleaving only the probe strand. This results in target release, enabling subsequent binding to and cleavage of another probe molecule. When the probe is conjugated onto AuNPs, complete cleavage from the AuNP surface produces a detectable 
signal in high ionic strength environments as the nanoparticles undergo aggregation. With such enzyme-assisted amplification, target detection can occur with a very low $\mathrm{nM}$ detection limit within 15 minutes. The extent of DNA loading on the AuNP surface plays an important role in the efficiency of DNA hybridization and aptamer-target assembly. Many studies have shown that high surface-coverage is associated with steric hindrance, electrostatic repulsive interactions and elevated surface salt concentration, whereas low surface-coverage can result in nonspecific binding of oligonucleotides to the particle surface. In chapter five, we investigated DNA surface coverage effects, and apply this optimization in conjunction with a highly-specific aptamer to develop a sensitive colorimetric sensor for rapid cocaine detection based on the inhibition of nuclease enzyme activity. 


\section{TABLE OF CONTENT}

\section{CHAPTER}

PAGE

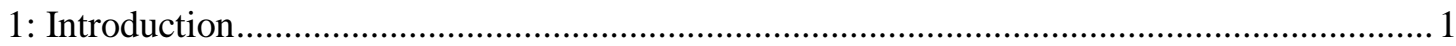

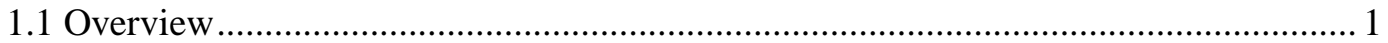

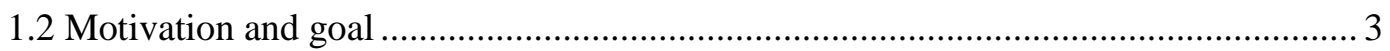

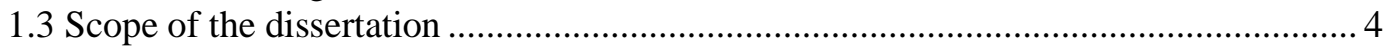

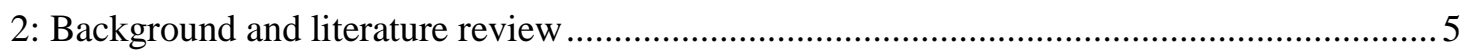

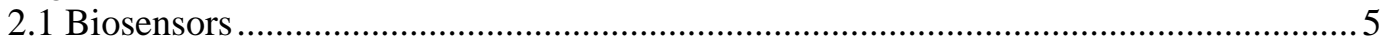

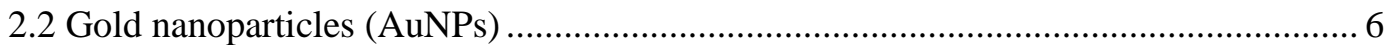

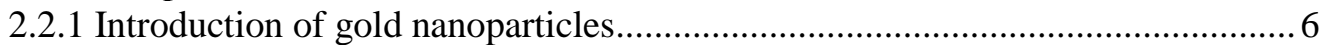

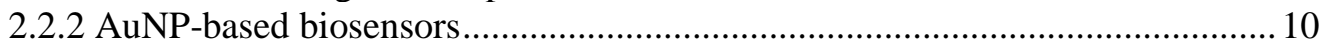

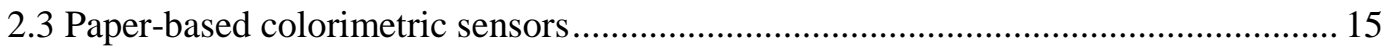

2.4 Dihydronicotinamide adenine dinucleotide, dehydrogenases, and inhibitors ............ 17

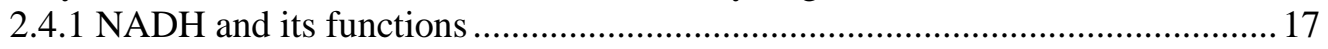

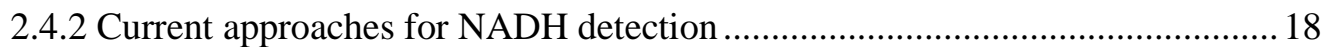

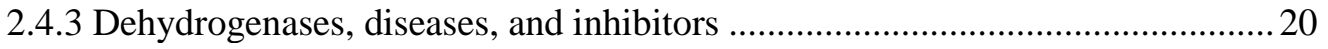

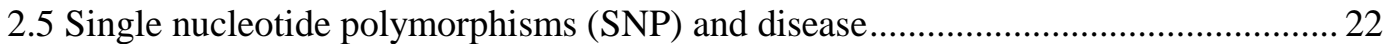

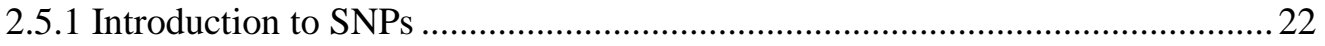

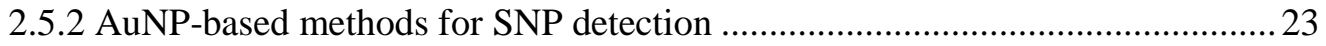

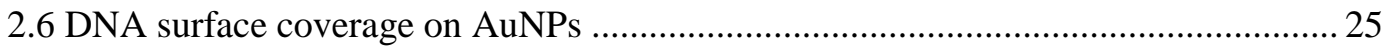

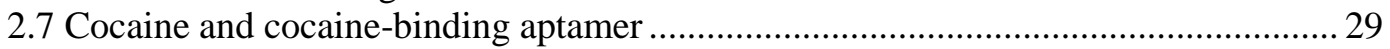

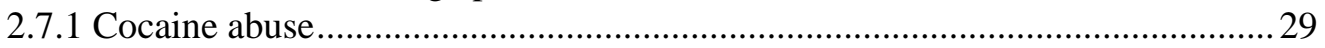

2.7.2 Current methods for cocaine detection.........................................................29

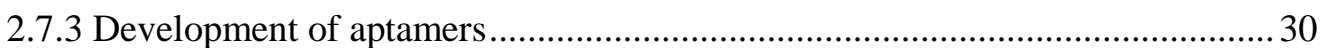

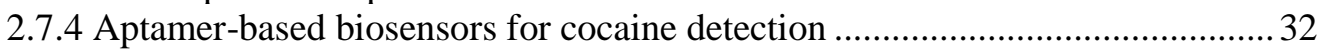

3: AuNP-based chemical sensor: A paper-based device for rapid visualization of NADH based on dissolution of gold nanoparticles ........................................................ 36

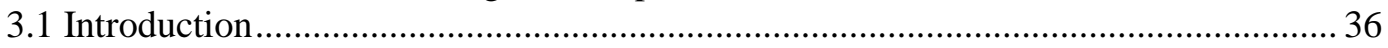

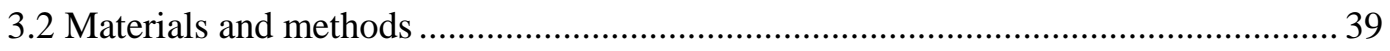

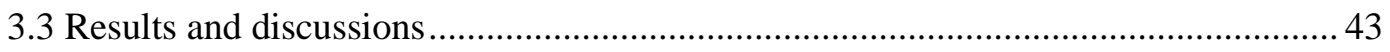

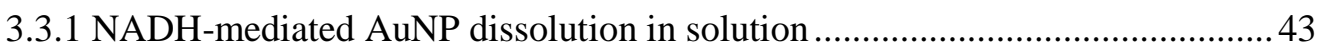

3.3.2 NADH-mediated AuNP dissolution on paper .............................................. 48

3.3.3 Using paper-based device to monitor NADH-related enzymatic reaction .........55

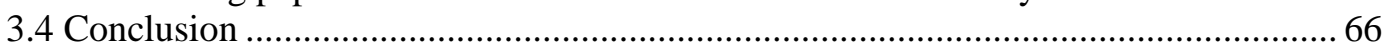

4: AuNP-based DNA biosensor: A colorimetric sensor based on Exo III-assisted target recycling for single nucleotide polymorphisms (SNP) detection..........................6 68

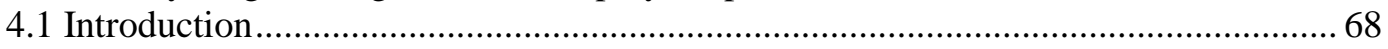

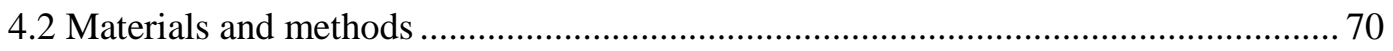

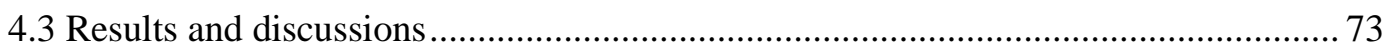

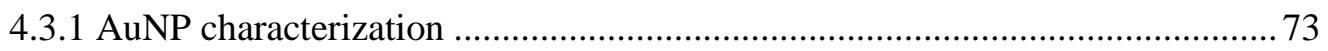

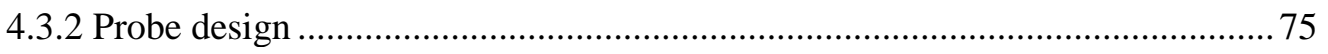

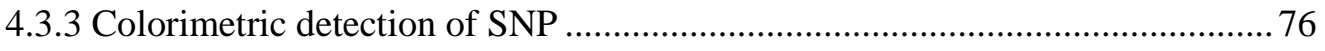

4.3.4 Human apurinic/apyrimidinic endonuclease 1 (APE 1) ................................... 79 


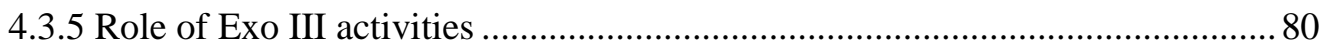

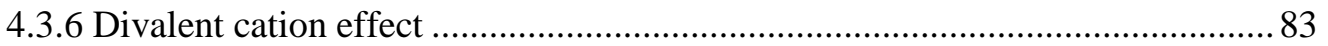

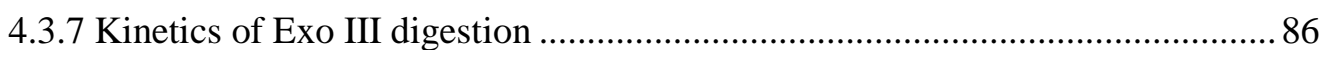

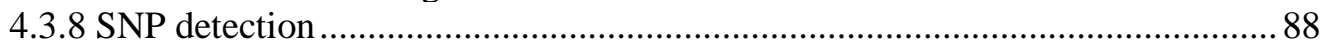

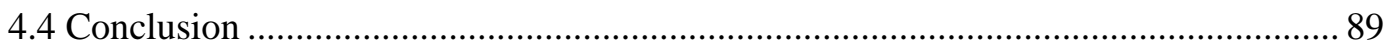

5: Effect of DNA Surface coverage on the performance of aptamer-based sensor platform

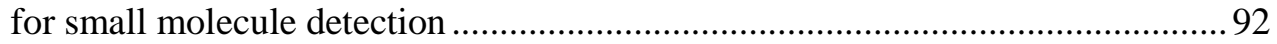

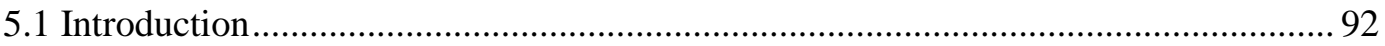

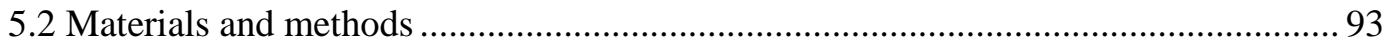

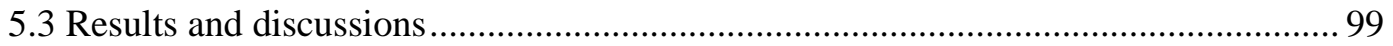

5.3.1 Evaluation of OliGreen fluorescence assay for the characterization of DNA

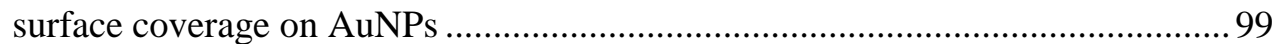

5.3.2 Development of a simple assay based on ligand-exchange strategy to rapidly alter DNA surface coverage on AuNPs ................................................ 106

5.3.3 Development of a colorimetric detection of cocaine based on Exo IIIinhibited digestion on target-aptamer complex ................................................. 114

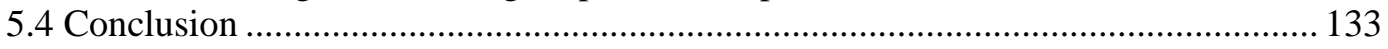

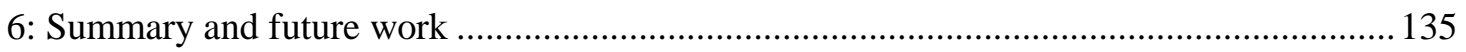

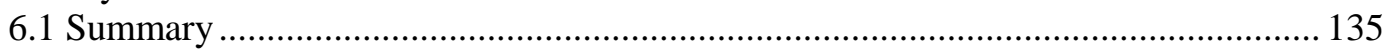

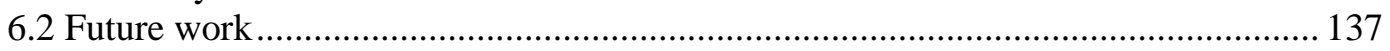

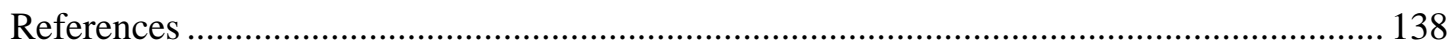

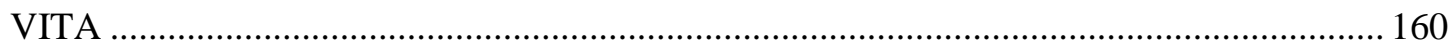




\section{LIST OF FIGURES}

FIGURE

PAGE

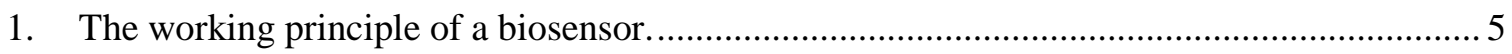

2. The Lycurgus Cup shows a different color depending on whether it is illuminated externally (left) or internally (right) (The British Museum) .............................................. 6

3. Schematic of AuNP formation via citrate reduction .............................................................. 7

4. Preparation of TOAB-stabilized AuNPs using the Brust-Schiffrin method............................ 7

5. SPR in AuNPs (left) results in absorbance of blue-green wavelengths and reflection of red wavelengths, as seen in a photo of a citrate-capped solution of 13-nm-diameter AuNPs (right)

6. The color of solutions of well-dispersed AuNPs shifts from red to blue as the diameter of the nanoparticles increases.

7. TEM images of (a) gold nanospheres, (b) nanorods, and (c) nanostars. (d) UV-Vis absorption spectra of all three gold nanoparticles ............................................................... 10

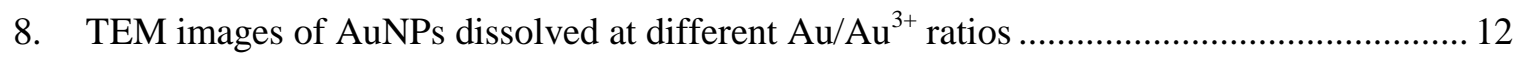

9. AuNP-based colorimetric method for differentiating between single- and doublestranded oligonucleotides....

10. Schematic representation of colorimetric detection of DNA using ssDNA-modified AuNPs

11. Interconversion between $\mathrm{NADH}$ and its oxidized form $\left(\mathrm{NAD}^{+}\right)$

12. Synthesis of thiolate DNA-AuNP conjugates

13. Chemical structure of cocaine

14. Three-way junction structure of the cocaine-bound MNS-4.1 aptamer

15. Colorimetric visualization of NADH based on AuNP dissolution on a paper-based device

16. Characterization of the concentration and size of these synthesized AuNPs. .44

17. Size characterization of the freshly prepared AuNPs. .45

18. Time course of AuNP dissolution in $\mathrm{Au}^{3+}{ }_{-} \mathrm{CTAB}$ solution. 46

19. The effect of $\mathrm{Au}^{3+}$ concentration on AuNP dissolution in CTAB 47 
20. The effect of NADH concentration on AuNP dissolution

21. UV-Vis spectrum of the AuNP solution before and after vacuum filtration

22. Dissolution of AuNP-coated MCE films at different $\mathrm{Au}^{3+}$ concentrations

23. Time course of the reaction on AuNP-coated MCE filter paper in the absence and presence of $200 \mu \mathrm{M}$ NADH in $200 \mu \mathrm{M} \mathrm{Au}{ }^{3+}$-CTAB solution .

24. Colorimetric detection of NADH on AuNP-coated MCE filter paper

25. Optimization of GDH catalyzed reactions by monitoring the absorbance of NADH at $340 \mathrm{~nm}$

26. Glucose detection in $400 \mu \mathrm{M} \mathrm{Au}{ }^{3+}-\mathrm{CTAB}$ after a 20 -minute reaction.

27. AuNP dissolution limited by lateral diffusion due to reactant escape either with or without a wax-circled test zone

28. A more even distribution of $\mathrm{Au}^{3+}-\mathrm{CTAB}$ resulted by using an added layer of absorbent pad material.

29. The performance of dry versus wet wax-circled films in conjunction with a dry or wet cotton absorbent layer

30. A simple paper-based device for colorimetric detection of NADH in a microliter-scale sample in less than 4 minutes.

31. Time course of the reaction in $25 \mu \mathrm{L}$ samples at room temperature on a paper-based device in the absence and presence of $200 \mu \mathrm{M}$ NADH in a $200 \mu \mathrm{M} \mathrm{Au}^{3+}$-CTAB solution... 59

32. Successful detection of NADH in $25 \mu \mathrm{L}$ samples in under 4 minutes at room temperature on a paper-based device

33. Successful detection of GDH produced NADH in $25 \mu \mathrm{L}$ samples in under 4 minutes at room temperature on a paper-based device

34. Detecting NADH with and without cell lysate using our paper-based device at room temperature

35. Detecting NADH production by GDH with our paper-based device at room temperature in the absence and presence of $20 \%$ E. coli cell lysate

36. $\mathrm{Hg}(\mathrm{II})$ inhibition effect on GDH activity in homogenous solution

37. Screening of $\mathrm{Hg}(\mathrm{II})$-mediated inhibition of GDH-driven NADH production at different concentrations.

38. Different ions inhibitory effect on GDH activity in homogenous solution 
39. Screening the inhibitory effects of various heavy metal ions on GDH-driven NADH production

40. Characterization of the concentration and size of these synthesized AuNPs ........................ 73

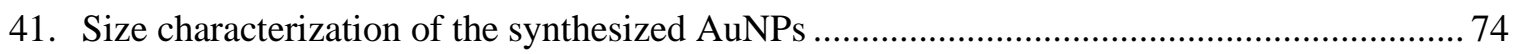

42. Scheme for CP-modified AuNPs, CP/PM and CP/SM duplexes ........................................... 75

43. Scheme for AuNP-based, Exo III-amplified colorimetric SNP detection.............................. 77

44. AuNP-based, Exo III-amplified colorimetric SNP detection within 15 minutes at room

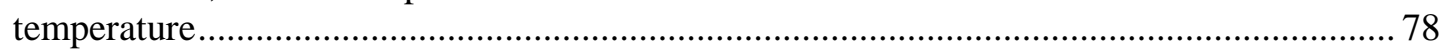

45. CP-modified AuNPs remained the protection against salt-induced aggregation even after 4-hour treatment with APE 1, which lacks exonuclease activity ........................................... 80

46. SNP discrimination achieved through the endonucleolytic incision of either Exo III or APE 1

47. The influence of $\mathrm{Ca}^{2+}$ concentration on target discrimination (A) and detection time (B).... 84

48. The influence of $\mathrm{Mg}^{2+}$ concentration on target discrimination (A) and detection time (B)... 85

49. Determination of Exo III kinetics in the AuNP-attached CP system 86

50. The dynamic range of the AuNP-based, Exo III-amplified sensor (10 nM - $100 \mathrm{nM})$ after a 10 -minute reaction.

51. A detection limit of $2 \mathrm{nM}(\mathrm{S} / \mathrm{N}>3)$ achieved by performing the reaction at $4{ }^{\circ} \mathrm{C}$ for 4 hours

52. The generality of mismatch detection of the AuNP-based, Exo III-amplified sensor

53. Scheme of DTT displacement of AuNP-conjugated fluorescein-labeled DNA strands.

54. Characterization of the surface coverage of NoX-FAM DNA on AuNPs .... 101

55. Characterization of the surface coverage of 1X-FAM DNA on AuNPs 102

56. Characterization of the surface coverage of 2X-FAM DNA probe. 103

57. Characterization of the DNA surface coverage of NoX No-T8 DNA and 1-X No-T8 DNA

58. Scheme of DTT displacement of AuNP-unlabeled DNA strands and the characterization of surface coverage of released DNA using OliGreen dye 106

59. The AuNP-based, Exo III-assisted target recycling (EATR)-based colorimetric detection of DNA. 108 
60. Scheme of regulating DNA surface coverage on AuNP by using mercaptohexanol (MCH)

61. Effect of MCH concentration on the adjusted DNA surface coverage .............................. 110

62. Effect of Exo III activity on the MCH-regulated DNA surface coverage ............................ 110

63. Scheme of adjusting DNA surface coverage on AuNP by using dithiothreitol (DTT) ........ 112

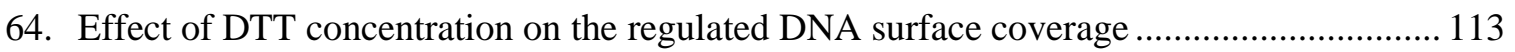

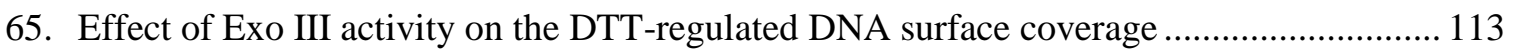

66. Time-course of 38-GC digestion with Exo III in the absence or presence of cocaine ......... 115

67. Engineering split aptamer 38-GC from its single cocaine aptamer.................................. 116

68. Exo III digestion on split 38-GC with and without $250 \mu \mathrm{M}$ cocaine in Tris buffer ( $\mathrm{pH} 7.4$ )

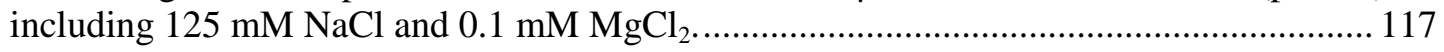

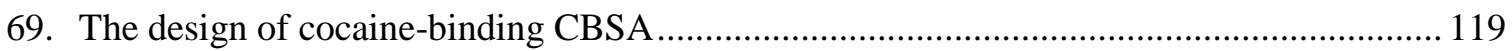

70. Structure of the cocaine unbound and bound CBSA-5325-AT aptamer .............................. 119

71. Exo III digestion on CBSA 5325-AT with and without $250 \mu \mathrm{M}$ cocaine in Tris buffer

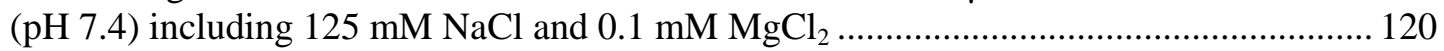

72. Engineering CBSA 5325-XT aptamer ("X” stands for abasic site) from CBSA 5325 ....... 121

73. Exo III digestion on CBSA 5325-XT with and without $250 \mu \mathrm{M}$ cocaine in Tris buffer

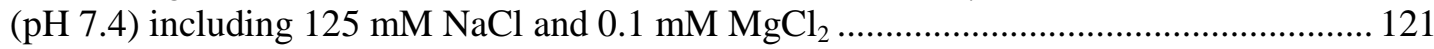

74. Working principle of AuNP-reported, Exo III-inhibited colorimetric detection of cocaine 122

75. Proof of concept of AuNP-reported, Exo III-inhibited colorimetric detection of cocaine... 123

76. Effect of the DTT-regulated DNA surface coverage on Exo III activity for cocaine

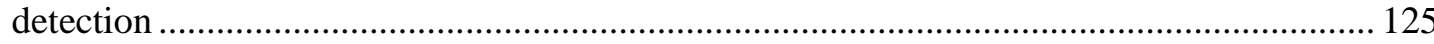

77. Effect of the MCH-regulated DNA surface coverage on Exo III activity for cocaine

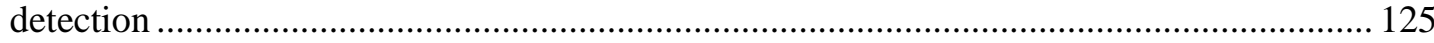

78. Effect of $\mathrm{MgCl}_{2}$ concentration and Exo III unit on the MCH-regulated DNA for cocaine

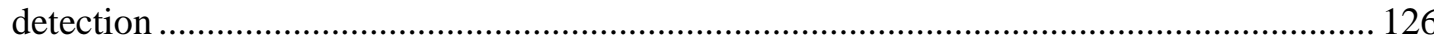

79. Effect of long CBSA aptamer fragment DNA on cocaine detection................................. 128

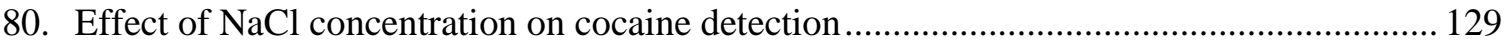

81. Effect of $\mathrm{MgCl}_{2}$ concentration on the cocaine detection............................................... 130 
82. Effect of long CBSA fragment concentration on cocaine detection

83. Effect of Exo III concentration effect on the cocaine detection .......................................... 131

84. Calibration curve of colorimetric detection of cocaine under optimized experimental

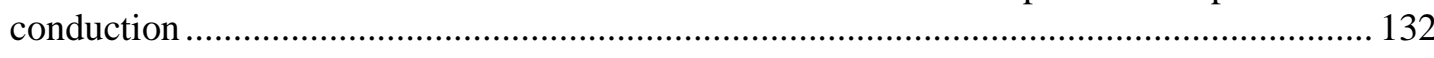

85. Engineering new CBSA aptamers to achieve a fast reaction with a better selectivity ......... 133 


\section{LIST OF ABBREVIATIONS}

Abs

ALDH

AMD

AP

AuNP

AuNR

AuNS

$\beta$

BP

CE

CL

CNS

Coc

CP

$\delta$

${ }^{\circ} \mathrm{C}$

DI

DNA

DNA-AuNPs

DNase I

dNMP

dNTP

ddNTP

dsDNA absorbance

aldehyde dehydrogenases

Age-related Macular Degeneration

apurinic endonucleolytic

gold nanoparticle

gold nanorod

gold nanostar

beta

binding pocket

capillary electrophoresis

chemiluminescence

central nervous system

cocaine

capture probe

delta

degrees Celsius

deionized water

deoxyribonucleic acid

DNA modified gold nanoparticles

Deoxyribonuclease I

deoxynucleotides monophosphate

deoxynucleotide triphosphate

dideoxynucleotide triphosphate

double-stranded DNA 


\begin{tabular}{|c|c|}
\hline DTT & Dithiothreitol \\
\hline ELISA & enzyme-linked immunosorbent assay \\
\hline EMIT & enzyme multiplied immunoassay technique \\
\hline Exo III & Exonuclease III \\
\hline FAM & Fluorescein \\
\hline FDA & Food and Drug Administration \\
\hline FPIA & fluorescence polarization immunoassay \\
\hline FRET & fluorescence resonance energy transfer \\
\hline $\mathrm{g}$ & gram \\
\hline GC/MS & gas chromatography/mass spectrometry \\
\hline GDH & glucose dehydrogenase \\
\hline h & hour \\
\hline HIV & human immunodeficiency virus \\
\hline HPLC & high-performance liquid chromatography \\
\hline IMP & inosine monophosphate \\
\hline IMPDH & inosine 5'-monophosphate dehydrogenase \\
\hline $\mathrm{L}$ & liter(s) \\
\hline LDH & lactate dehydrogenase \\
\hline$\lambda$ & lambda \\
\hline $\mathrm{m}$ & milli \\
\hline M & moles per liter \\
\hline MB & methylene blue \\
\hline MCE & mixed cellulose ester \\
\hline $\mathrm{MCH}$ & 6-Mercapto-1-hexanol \\
\hline MCU & mercaptoundecanol \\
\hline
\end{tabular}




\begin{tabular}{|c|c|}
\hline MCP & mercaptopropanol \\
\hline ME & mercaptoethanol \\
\hline$\mu$ & micro \\
\hline $\min$ & minute(s) \\
\hline mol & mole(s) \\
\hline MNPs & magnetic nanoparticles \\
\hline MRI & magnetic resonance imaging \\
\hline MS & mass spectrometry \\
\hline MUD & mercaptoundecanol \\
\hline NADH & dihydronicotinamide adenine dinucleotide \\
\hline $\mathrm{NAD}^{+}$ & nicotinamide adenine dinucleotide \\
\hline NSDUH & National Survey on Drug Use and Health \\
\hline OEG & oligo(ethylene glycol) \\
\hline OG & OliGreen \\
\hline PKU & phenylketonuria \\
\hline PM & perfectly matched \\
\hline$\%$ & percentage \\
\hline RIA & radioimmunoassay \\
\hline RT & room temperature \\
\hline s & second(s) \\
\hline SAM & Self Assembled Monolayers \\
\hline SERS & Surface-Enhanced Raman Scattering \\
\hline SGD & SYBR Gold \\
\hline SM & single-base mismatched \\
\hline SNPs & single nucleotide polymorphisms \\
\hline
\end{tabular}




$\begin{array}{ll}\text { SPR } & \text { Surface Plasmon Resonance } \\ \text { ssDNA } & \text { single-stranded DNA } \\ \text { TEM } & \text { transmission electron microscopy } \\ \text { Tris } & \text { Tris(hydroxymethyl)aminomethane } \\ \text { UV-Vis } & \text { ultraviolet-visible } \\ \text { w/ } & \text { with } \\ \text { w/o } & \text { without } \\ \text { XMP } & \text { xanthosine 5'-monophosphate }\end{array}$




\section{CHAPTER 1: Introduction}

\subsection{Overview}

Biosensors are useful tools for monitoring patient health because they can detect and analyze diverse targets, such as proteins, DNA, and small molecules representing disease biomarkers, toxins, drugs and their metabolites, environmental pollutants, agrichemicals, and antibiotics with high sensitivity and specificity.

Target detection is typically performed with either quick, qualitative methods or accurate, quantitative methods. Qualitative readouts are usually desirable in many applications, particularly in the context of on-site diagnostics where treatment could be dictated by a simple "yes/no" or "normal/abnormal" response. Due to increasing health care costs, there is a growing demand to be able to monitor patient health through point-of-care-testing, necessitating the development of simple, rapid, portable, and inexpensive detection assays.

In addition to being highly sensitive and selective, an ideal biosensor for point-of-care applications should be label-free, cost-effective, involve minimum sample preparation, and have the potential for miniaturization. Colorimetric assays offer one useful option, based on a visible color change induced by target binding or complex formation. These assays deliver results that can be directly observed by naked eye and do not require sophisticated instruments, making them a potentially simple, low-cost approach to field analysis and point-of-care diagnosis. Paper-based colorimetric detection has drawn particular attention in the realm of biosensor development for analytical, clinical and environmental analysis, with several advantages including versatility, disposability, low cost, and ease of fabrication. Pregnancy tests are one well-known example, providing a swift and simple qualitative "yes/no" answer in the form of a color change on the paper test strip. Urine test strips are another example, providing qualitative or semi-quantitative detection of multiple analytes at the same time, including ketones, glucose, protein and $\mathrm{pH}$ value in a few minutes. 
Gold nanoparticles (AuNPs) have been intensively studied due to a distinctive optical property known as surface plasmon resonance (SPR) absorption. Depending on their size, shape, composition, and degree of aggregation, AuNPs can absorb resonance light at various wavelengths and vary in color. On the basis of these properties, AuNPs have been successfully applied as colorimetric reporters for the detection of various substances, including DNA, metal ions, or proteins.

Unmodified AuNPs have been used for DNA detection based on the differences in electrostatic properties between single-stranded (ss) DNA, double-stranded (ds) DNA, and AuNPs. ssDNA adsorbs onto AuNPs nonspecifically, which protects them from aggregating in high ionic strength environments, while dsDNA or folded ssDNA does not prevent such aggregation, causing a red-to-blue color change. Unmodified AuNPs have also been widely used in small-molecule detection assays based on the biocatalytic growth process, which results in a larger size with red-shifted wavelengths and higher SPR absorption. In contrast, when AuNPs decrease in size during the dissolution process, this produces blue-shifted or shorter wavelengths, and ultimately the completely disappearance of the nanoparticles. However, only inorganic targets have been detected based on the dissolution of AuNPs.

Thiolated DNA can be assembled onto the surface of AuNPs via well-established Au-S chemistry, and these DNA strands can undergo hybridization and dissociation from complementary strands while remaining attached to the gold surface. Thiolated DNA-modified AuNPs can remain well dispersed in an aqueous solution and remain red in color. When a DNA or small molecule linker is subsequently added into the solution, the DNA-AuNPs form a network and undergo aggregation, producing a color change to purple or blue due to the resulting distance change. DNA-functionalized AuNPs have thus been widely used for DNA and smallmolecule detection. 


\subsection{Motivation and goal}

The major objective of the research described in this dissertation was to develop low cost, low sample volume, highly sensitive and specific AuNP-based colorimetric sensor platforms for the detection of DNA and small molecules. Critically, the color changes obtained with AuNPs can be maintained on paper-based substrates. With this in mind, we propose an instrument-free approach in Chapter three for the detection of NADH with a sensor constructed on a paper substrate, based on the target-induced inhibition of AuNP dissolution. The successful detection of this one molecule opens the door to numerous possibilities for dehydrogenase characterization, because $\mathrm{NAD}^{+} / \mathrm{NADH}$ are essential cofactors for more than 300 dehydrogenase enzymes.

In sensor development, sensitivity is an important parameter for evaluating performance. To improve the sensitivity of detection, various signal amplification strategies can be employed, including those based on the use of nanomaterials. Colorimetric detection based on AuNPs is innately highly sensitive due to the high extinction coefficients of their surface plasmon absorption bands, which are more than 1,000 times higher than those of organic dyes. To further increase the sensitivity of our hybridization-based assay for DNA detection, we developed an enzyme-assisted target recycling (EATR) strategy. This assay is based on the principle that nuclease enzymes recognize probe-target complexes, cleaving only the probe strand. This results in target release, enabling subsequent binding to and cleavage of another probe molecule. When the probe is conjugated onto AuNPs, complete cleavage from the AuNP surface produces a detectable signal in high ionic strength environments as the nanoparticles undergo aggregation. With such enzyme-assisted amplification, target detection can occur with a very low detection limit. As described in Chapter Four, we have applied such an EATR-based colorimetric assay for detecting single-nucleotide mismatches in a target DNA with DNA-functionalized AuNPs.

The extent of DNA loading on the AuNP surface plays an important role in the efficiency of DNA hybridization and aptamer-target assembly. High surface density leads to increasing steric 
and electrostatic repulsion between the DNA probe and target, causing low hybridization efficiency and subsequently a longer reaction time. At low surface density, steric hindrance can be greatly reduced, thus enhancing hybridization efficiency for target binding, but the probes are also more likely to exist as flattened structures as a result of strong interactions with the AuNP surface. Therefore, optimization of probe density is necessary to ensure sufficient hybridization efficiency with a rapid reaction time, and also to improve assay sensitivity. In Chapter Five, we investigated DNA surface coverage effects, and apply this optimization in conjunction with a highly-specific aptamer to develop a colorimetric sensor for cocaine detection based on the inhibition of nuclease enzyme activity.

\subsection{Scope of the dissertation}

Chapter two provides a general literature review of the historical background of AuNP synthesis; size-, shape- and distance-dependent SPR absorption; and the current application of AuNPs in colorimetric detection. This chapter also reviews existing detection methods for the three model targets - NADH, DNA single-nucleotide polymorphisms (SNP), and cocainestudied in this dissertation. Paper-based colorimetric sensor development and analysis of DNA surface coverage on the gold surface are also reviewed. Chapter three details the fabrication of a paper-based device for rapid visual detection of NADH based on dissolution of unmodified AuNPs, and demonstrates the application of this paper-based device for monitoring glucose dehydrogenase $(\mathrm{GDH})$ production of NADH and screening of GDH inhibitors. Chapter four describes the EATR assay for nuclease-assisted SNP detection with thiolated DNA-modified AuNPs. Chapter five details a simple method to control DNA surface coverage on AuNPs and tests the effects of surface coverage on DNA and small molecule detection. Chapter 6 gives a summary of the dissertation and proposes a future plan. 


\section{CHAPTER 2: Background and literature review}

\subsection{Biosensors}

According to the International Union of Pure and Applied Chemistry's (IUPAC) definition, ${ }^{1}$ a biosensor may be defined as a device incorporating a bioreceptor (e.g., enzyme, DNA, microorganism, tissue or whole cells) to detect an analyte with a suitable physicochemical transducer (Figure 1). Typically, the analyte specifically binds to the bioreceptor, and this binding event is then converted into a quantifiable readout, such as an electrical, thermal or optical signal that is proportional to the concentration of the analyte. A biosensor with a sufficiently sensitive transducer can directly detect analytes even in the milieu of complex matrices.

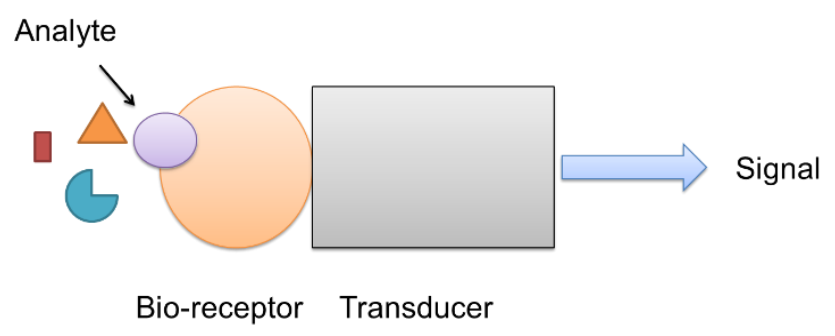

Figure 1. The working principle of a biosensor.

Biosensors can be classified within two broad categories based on the nature of their bioreceptor and transducer components. Bio-recognition elements can be categorized as enzyme sensors, immunosensors, DNA sensors, or cell/tissue-based sensors, whereas transducers can be classified as optical, piezoelectric, or electrochemical biosensors. ${ }^{2}$ Optical sensors can function based on diverse principles, including absorption at ultraviolet, visible, or infrared wavelengths; fluorescence; gel electrophoretic mobility; and surface plasmon resonance (SPR). Colorimetric sensing in particular has important advantages over other detection methods. For example, readouts based on visible color readouts or color changes can be directly observed by the naked eye for field analysis and point-of-care diagnosis without requiring sophisticated instrumentation. 


\subsection{Gold nanoparticles (AuNPs)}

\subsubsection{Introduction of gold nanoparticles}

Nanoparticles are widely used in biosensor development. Gold nanoparticles (AuNPs), known as colloidal gold, are fluid suspensions of gold particles with diameters ranging from 1100-nm that have been protected with a capping reagent. AuNPs are one of the most studied nanomaterials due to their distinctive optical and electronic properties and good biocompatibility. ${ }^{3-6}$ The optical properties of AuNPs have been known since early in human history; perhaps the most well-known example is the Lycurgus Cup from the $4^{\text {th }}$ century AD at the British Museum in London, whose glass appears red in transmitted light and green in reflected light. Detailed analysis revealed that the presence of nanometer-size metal crystals of $\mathrm{Ag}$ and $\mathrm{Au}$ gave the cup its unusual optical properties (Figure 2).

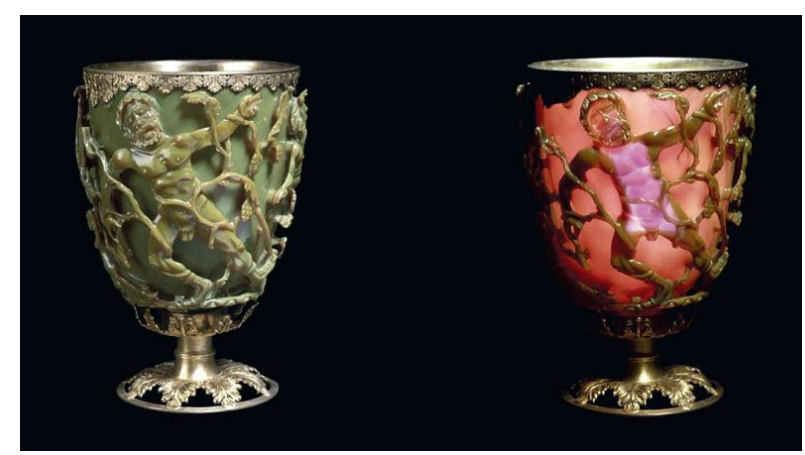

Figure 2. The Lycurgus Cup shows a different color depending on whether it is illuminated externally (left) or internally (right) (The British Museum).

The first chemical synthesis of gold colloid was reported by Michael Faraday in $1857 .^{7}$ The deep red solution of colloidal gold was obtained by the reduction of an aqueous chloroauric $\left(\mathrm{AuCl}_{4}{ }^{-}\right)$solution by phosphorus in $\mathrm{CS}_{2}$, a two-phase system. ${ }^{7}$ In the modern era, AuNPs have become a hot topic, and more controllable synthesis methods and applications in diverse nanosystems had been developed. A quick search of Web of Science with the keywords "gold nanoparticles", and "detection"' reveals more than 7,000 journal articles and reviews from between 2005 and 2015 related to nanoparticle synthesis, modification, and properties. 
Many different techniques have been developed for the synthesis of AuNPs through the chemical reduction of $\mathrm{HAuCl}_{4}$ in the presence of capping reagents. The most frequently utilized technique is the citrate method reported by Turkevitch et al. in $1951^{8}$ for synthesizing AuNPs of up to $150-\mathrm{nm}$ in diameter. In this approach, $\mathrm{Au}$ (III) salt is first reduced to $\mathrm{Au}(\mathrm{I})$ ions, after which $\mathrm{Au}(0)$ species are formed through a disproportionation reaction. These activated $\mathrm{Au}(0)$ species are thermodynamically unstable and act as the center of nucleation for the further reduction of $\mathrm{Au}^{+}$ions. This growth process gives rise to well-dispersed AuNPs (Figure 3). In this method, citrate molecules act as both a reducing and capping reagent. The size of the AuNPs can be finely and easily tuned by adjusting the $\mathrm{HAuCl}_{4}$ : citrate ratio, temperature, or solution $\mathrm{pH}$.

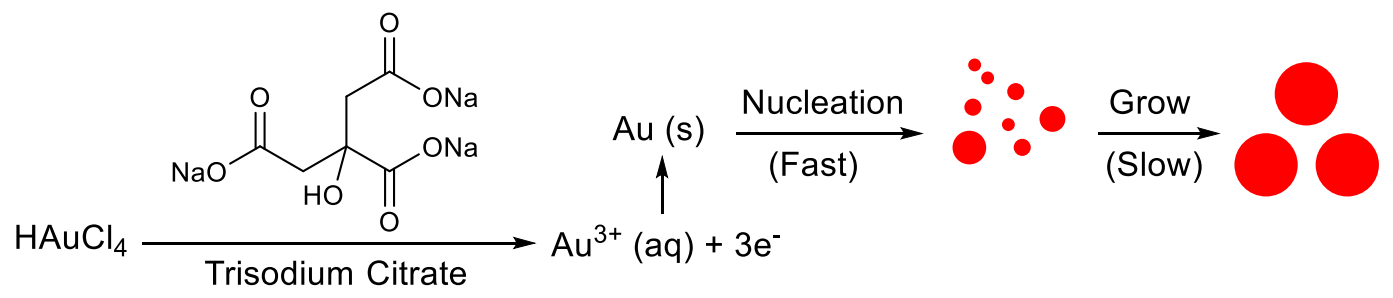

Figure 3. Schematic of AuNP formation via citrate reduction.

A second commonly-used procedure called the Brust-Schiffrin method, first reported in $1994,{ }^{9}$ uses a biphasic toluene-water system under ambient conditions to achieve synthesis of small AuNPs (1-5-nm diameter). Metal nucleation centers are first formed inside micelles of tetrabutylammonium bromide (TOAB) within the organic solvent, where the metal ions are reduced by sodium borohydride $\left(\mathrm{NaBH}_{4}\right)$ in the presence of a thiol capping ligand (Figure 4). The size of the AuNPs can be controlled by adjusting the thiol molecule: gold salt ratio, where higher ratios produce smaller particles. The resulting AuNPs can easily be re-dispersed in many organic solvents, and are stable for a long time.

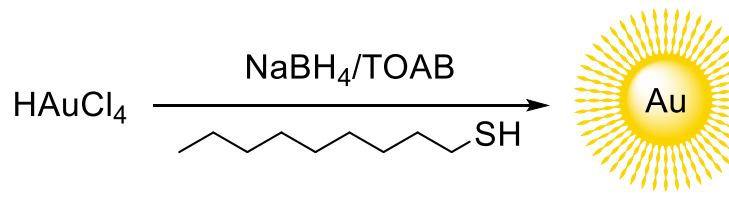

Figure 4. Preparation of TOAB-stabilized AuNPs using the Brust-Schiffrin method. 


\section{Non-spherical AuNPs}

Non-spherical gold nanoparticles shaped like rods, ${ }^{10}$ stars, ${ }^{11}$ prisms, ${ }^{12}$ cubes, ${ }^{13}$ and shells ${ }^{14}$ each exhibit intriguing shape-dependent plasmonic properties. Significant progress has been made in the synthesis of non-spherical gold nanoparticles via seed-mediated growth methods. ${ }^{10,12}$ Gold nanorods (AuNRs) can be produced by treating AuNP seeds (diameter $\sim 3 \mathrm{~nm}$ ) with a solution containing gold, cetyltrimethylammonium bromide (CTAB) and ascorbic acid (a mild reducing agent), which causes the seed to grow via a process of nucleation into an elongated nanorod. ${ }^{10}$ Surfactants in the growth solution form a bilayer that helps to direct growth of the nanoparticles. The aspect ratio (length divided by width) of the AuNRs can be controlled by altering the concentration of silver nitrate in the growth solution. ${ }^{15} \mathrm{Au}$ nanostars (AuNS) are branched nanostructures with a central core and several protruding arms with sharp tips, which can also be synthesized by the seed growth method in a concentrated solution of poly(vinylpyrrolidone) (PVP) and dimethylformamide (DMF). ${ }^{11}$

\section{Surface plasmon resonance and coloring}

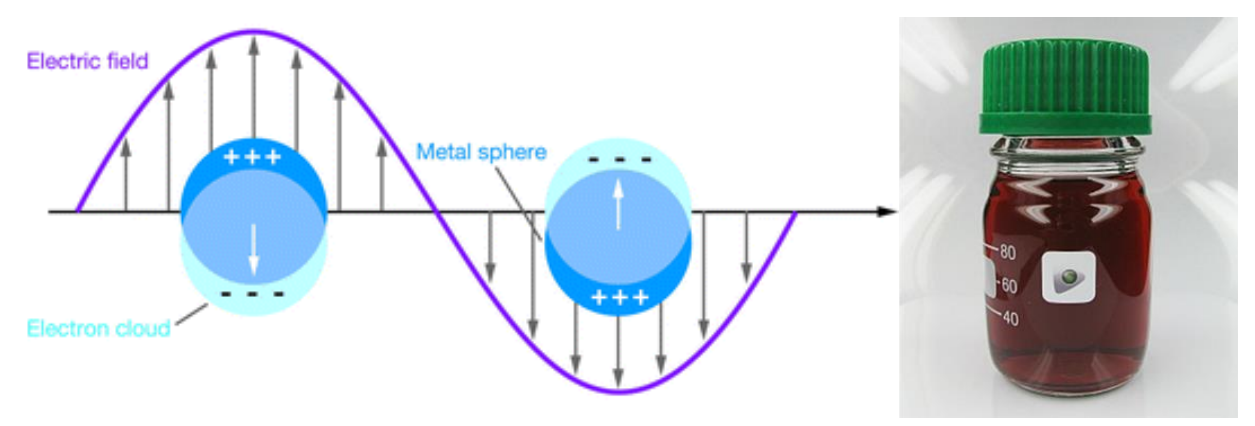

Figure 5. SPR in AuNPs (left) results in absorbance of blue-green wavelengths and reflection of red wavelengths, as seen in a photo of a citrate-capped solution of 13-nm-diameter AuNPs (right).

The exclusive optical properties of AuNPs arise from localized surface plasmon resonance (SPR). ${ }^{16}$ When light irradiates a solution of dispersed metal nanoparticles, the electromagnetic frequency induces resonant coherent oscillation of the free electrons. When the frequency of the 
incident light matches the frequency of electron oscillation, specific wavelengths of light are absorbed (Figure 5). ${ }^{17}$ The oscillation frequency of AuNPs is usually in the visible region, giving rise to a strong color associated specifically with SPR-related absorption.

The diameter of AuNPs affects the surface plasmon absorption. 13-nm-diameter AuNPs usually produce an absorbance peak at green-blue wavelengths $(\sim 520 \mathrm{~nm})$ and reflect red wavelengths $(\sim 700 \mathrm{~nm})$, and such solutions, therefore show a rich red color. Increased particle size produces a wider peak due to loss of coherent electron motion, resulting in a spectral redshift towards longer wavelengths and broadening of the surface plasmon band. ${ }^{18}$ Accordingly, small AuNPs ( 4-nm diameter) show orange-red color with an absorbance peak at $504 \mathrm{~nm}$, whereas large AuNPs ( 100-nm diameter) exhibit purple color with an absorbance peak at $560 \mathrm{~nm}$ (Figure $6)$.

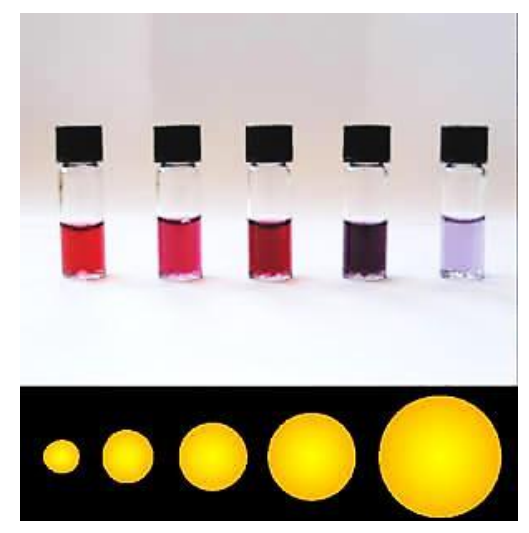

Figure 6. The color of solutions of well-dispersed AuNPs shifts from red to blue as the diameter of the nanoparticles increases.

Different shapes of gold nanoparticles exhibit different SPR absorption behavior (Figure 7). For example, AuNRs are brownish in color due to the existence of two plasmon bands: a transverse plasmon resonance in the visible range $(\sim 520 \mathrm{~nm})$ and a prominent longitudinal plasmon resonance in the near-infrared range $(700-800 \mathrm{~nm})$. The longitudinal SPR is typically red-shifted as the aspect ratio of the nanorod increases. ${ }^{18}$ AuNSs also typically display two local SPR bands, at 532 and $675 \mathrm{~nm}^{19}$ 

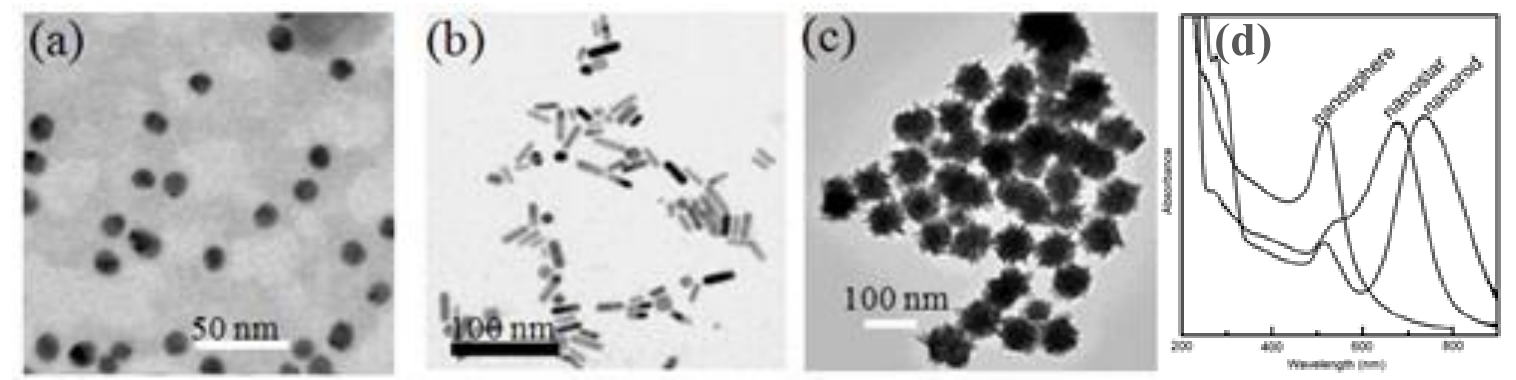

Figure 7. TEM images of (a) gold nanospheres, (b) nanorods, and (c) nanostars. (d) UV-Vis absorption spectra of all three gold nanoparticles.

\subsubsection{AuNP-based biosensors}

AuNPs can produce various colors and emit bright resonance light scattering of various wavelengths depending on their size, shape, composition and degree of aggregation, as well as effects from the surrounding environment. ${ }^{20-32}$ This means that highly sensitive colorimetric sensors can be designed by employing different mechanisms to control the size or separation of AuNPs. AuNPs also exhibit a high extinction coefficient (1000 times greater than that of organic dyes), large surface area, and are capable of easy surface modification. For these various reasons, AuNPs have been widely used as sensing element for colorimetric detection of targets including small molecules, ${ }^{20-24,26}$ DNA, ${ }^{28,29}$ inorganic ions, ${ }^{30}$ and metal ions. ${ }^{27,31,32}$

\subsubsection{Assays based on unmodified AuNPs}

\section{Enlargement of AuNPs}

AuNPs also exhibit intriguing active chemical properties. As described above, their size and shape can be chemically enlarged by seed-mediated growth under mildly reducing conditions, which makes it possible to tailor their optical, electronic and catalytic properties. ${ }^{24,25,33-36}$ To begin, small AuNPs are prepared as seeds. ${ }^{10}$ Reducing agents such as hydrogen peroxide, ${ }^{34}$ hydroxylamine, ${ }^{23,33} \mathrm{NADH},{ }^{22}$ flavonoids,${ }^{35}$ ascorbic acid, ${ }^{25}$ hydroquinone ${ }^{24}$ or neurotransmitters (e.g., dopamine $)^{37}$ are then added along with $\mathrm{AuCl}_{4}{ }^{-}$salt in the presence of a surfactant - typically $\mathrm{CTAB}^{22}$ or cetyltrimethylammonium chloride (CTAC) $)^{24,34}$ to promote growth of the AuNP seeds. A red-shifted visible wavelength and increased absorbance indicate successful AuNP growth. The 
extent of the enlargement of the gold seeds and the accompanying shift in optical absorbance is typically proportional to the amount of reducing reagent used. Catalytic growth of AuNP seeds has been coupled with several analytical methods such as absorption spectroscopy, 22, 34, 37-39 resonance light scattering, ${ }^{40}$ fluorescence spectroscopy, ${ }^{41}$ and electrochemistry ${ }^{24,42}$ in order to improve assay sensitivity. For example, electrocatalytic growth of AuNPs on an electrode surface greatly facilitated electron transfer and improved the sensitivity of the NADH detection. ${ }^{23,24}$

The AuNPs growth can also be combined with enzymatic reactions, a relatively new field that offers excellent prospects for the design of sensitive enzymatic assays for quantitative substrate detection. Enzyme including oxidases, hydroxylases, and $\mathrm{NAD}(\mathrm{P})^{+}$dependent dehydrogenase have been employed as effective biocatalysts for metal nanoparticle growth. For example, glucose oxidase has been used as a biocatalyst for in situ generation of $\mathrm{H}_{2} \mathrm{O}_{2}$, which in turn promotes growth of AuNP seeds. ${ }^{34}$ This in turn led to the development of an optical detection path for the sensing of glucose. Lactate/ethanol concentration can also be detected by NADH produced during the enlargement of AuNPs. ${ }^{22}$ These biosensing protocols mainly rely on the reduction of $\mathrm{AuCl}_{4}{ }^{-}$ by enzymatically-produced hydrogen peroxide or NADH as reducing agents to achieve AuNP enlargement and thereby generate a visible color readout.

\section{Dissolution of AuNPs}

AuNPs can be dissolved via chemical reactions in the presence of $\mathrm{CN}^{-} / \mathrm{O}_{2},{ }^{43-45}$ resulting in a uniform decrease in diameter. $\mathrm{CN}^{-}$detection was been reported based on the dissolution of fluorescein isothiocyanate (FITC)-capped AuNPs in the presence of cyanide ions, wherein AuNP dissolution led to the release of FITC molecules and increased fluorescence. ${ }^{46}$ However, $\mathrm{CN}^{-}$is highly toxic, and it is hard to ensuring adequate oxygenation. AuNP dissolution has also been reported using other ligand/oxidant pairs, such as $\mathrm{I}_{2} / \mathrm{I}^{-}$or $\mathrm{I}_{2} / \mathrm{SCN}^{-},{ }^{47}$ or $\mathrm{Cl}^{-} / \mathrm{H}_{2} \mathrm{O}_{2}{ }^{48}$ in an aqueous or non-aqueous medium ${ }^{49}$ to resize or reshape these particles. ${ }^{50}$ This completely avoids use of hazardous substances. For example, $\mathrm{Cl}^{-}$ions were converted to the corresponding chlorine 
radicals in the presence of UV light and thermal activation to oxidize gold clusters for the colorimetric detection of $\mathrm{HCl}^{48}$ It was found that $\mathrm{Cl}^{-}$could dissolve the AuNPs in an aqueous dispersion containing a strong oxidizing agent, such as $\mathrm{HNO}_{3}$ or $\mathrm{H}_{2} \mathrm{O}_{2}$ and thereby cause a color change. Huang et al. found that the addition of 2-mercaptoethanol (2-ME) could form $\mathrm{Au}(2-\mathrm{ME})_{2}$ complexes and accelerate the leaching of AuNPs in an ammonia-thiosulfate system. ${ }^{51}$ On the basis of this finding, a colorimetric, label-free, non-aggregation-based method was developed for the detection of $\mathrm{Pb}^{2+}$ ions in aqueous solution via the formation of $\mathrm{Pb}-\mathrm{Au}$ alloys on $\mathrm{AuNP}$ surfaces. Although $\mathrm{Pb}^{2+}$ ions were able to accelerate the reaction rate, the leaching process was still time-consuming ( 2 hours). In addition, 2-ME is toxic and easily oxidized in air.

Recently, mild oxidation of AuNPs has been achieved with surfactant solutions such as CTAB ${ }^{52-54}$ Aguirre et al. first observed that exposure to CTAB in aqueous solutions enabled reshaping of gold shells to form highly asymmetric gold rod- or bean-like structures at room temperature. ${ }^{14}$ Rodriguez-Fernandez et al. subsequently reported the sculpting of Au nanospheres via the oxidation of $\mathrm{Au}^{3+}$ in $\mathrm{CTAB}$ solution, and proposed that the charged $\mathrm{CTAB}$ micelles spatially directed the oxidation of nanoparticles. ${ }^{53}$ This eventually led to formation of smaller nanoparticles; the degree of oxidation is dependent on the ratio of $\mathrm{HAuCl}_{4}$ to $\mathrm{Au}$ seeds (Figure 8).

$$
\mathrm{AuCl}_{4}^{-}+2 \mathrm{Au}^{0}+2 \mathrm{Cl}^{-} \rightleftharpoons 3 \mathrm{AuCl}_{2}^{-}
$$

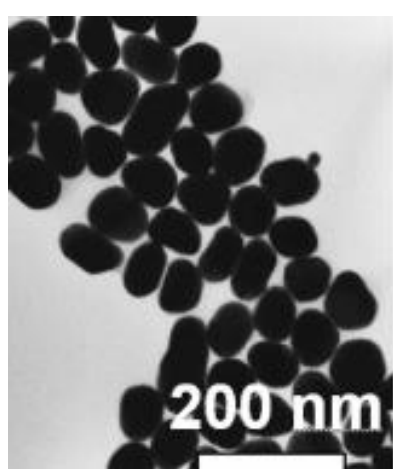

$3+$

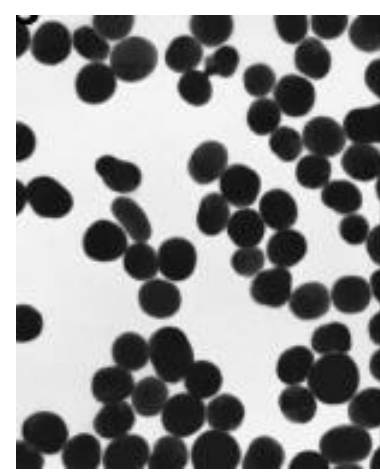

$1: 0.25$

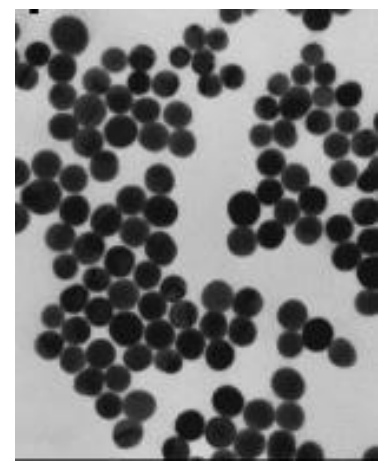

$1: 0.4$

Figure 8. TEM images of AuNPs dissolved at different $\mathrm{Au} / \mathrm{Au}^{3+}$ ratios. 
Other groups have also applied dissolution for the resizing or reshaping of AuNRs. ${ }^{50,55}$ Given that the optical properties of AuNRs are greatly affected by a small change in their shape and size, a variety of colorimetric methods have been developed based on wavelength changes in the absorption spectra of AuNRs. Colorimetric sensors for the detection of $\mathrm{Fe}^{3+}, \mathrm{Cr}^{6+}, \mathrm{Cu}^{2+}, \mathrm{H}_{2} \mathrm{O}_{2}$, $\mathrm{NO}_{2}{ }^{-}$and $\mathrm{I}^{-}$have been reported based on the oxidative etching of either AuNPs or AuNRs. ${ }^{48,51,56-}$

${ }^{62}$ For example, a colorimetric sensor was established for the detection of $\mathrm{Cr}^{6+}$ based on selective redox etching of AuNRs. The $\mathrm{Cr}^{6+}$ ions strongly oxidized the AuNRs and caused their aspect ratio to decrease, generating a blue shift in SPR absorption and a visible color change from bluishgreen or red to light red and then to colorless as the target's concentration increased. ${ }^{59} \mathrm{~A}$ similar method was reported involving the oxidative etching of AuNRs by ferric chloride at room temperature. The oxidative species $\left(\mathrm{Fe}^{3+}\right)$ and complexing agents $\left(\mathrm{Br}^{-}, \mathrm{Cl}^{-}\right)$cooperatively led to the chemical shortening of the AuNRs under mild conditions. ${ }^{56}$ However, this process required up to 5 hours. The addition of $I^{-}$greatly accelerated the etching rate, leading to a sharp decrease in the length of the AuNRs. The local SPR absorption peaks of the AuNRs shifted to shorter wavelengths with the decreased absorbance, and a change in solution color was observed as well, indicating that $\mathrm{I}^{-}$contributed to signal amplification. ${ }^{63}$ The color changes of unmodified AuNPs based dissolution can be visible to the naked eye. However, so far, only inorganic targets have been detected using these platforms.

\section{AuNP aggregation-based color change}

Free ssDNA can adsorb onto unmodified AuNPs due to the electrostatic effects of nitrogen atoms in the bases, protecting AuNPs from aggregating in a high ionic strength environment. In contrast, dsDNA cannot achieve such protection (Figure 9) ${ }^{64}$ Single-base mismatches have been detected by taking advantage of this difference in electrostatic properties between ssDNA and dsDNA. Unmodified AuNPs can also differentiate between folded and unfolded ssDNA. Fan et al. reported that ssDNA aptamers could protect unmodified AuNPs from aggregation in the 
presence of salt; however, upon binding with their target, $\mathrm{K}^{+}$ions, the aptamers folded into a Gquartet structure via intramolecular hydrogen bonds between guanines, and were no longer capable of adsorbing onto AuNPs. The addition of salt therefore resulted in aggregation of AuNPs that produced a red-to-purple color change. ${ }^{27}$ Water-soluble polymers have also been used to differentiate between ssDNA and dsDNA or folded DNA structures based on AuNP aggregation. Based on this concept, Xia et al. detected a broad range of targets, including DNA sequences, proteins, small molecules, and inorganic ions. ${ }^{28}$ They observed that positively-charged polymers could inhibit the ability of ssDNA to adsorb onto AuNPs, causing aggregation, whereas no such inhibition was observed with double-stranded or folded DNA structure.

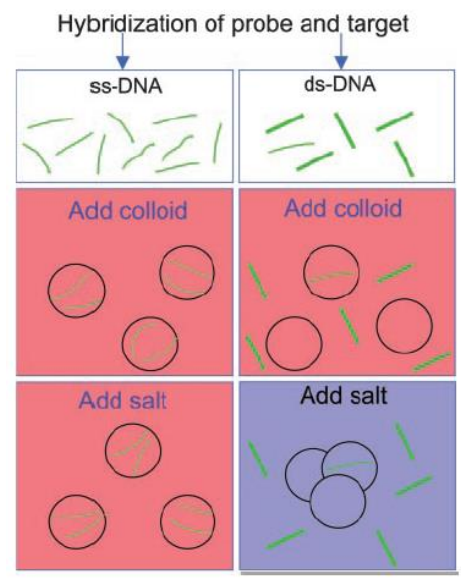

Figure 9. AuNP-based colorimetric method for differentiating between single- and doublestranded oligonucleotides.

\subsubsection{Assays based on functionalized AuNPs}

AuNPs are also attractive candidates for optical detection assays because of the ease with which they can undergo surface functionalization with molecules such as antibodies, ${ }^{65}$ peptides, ${ }^{66}$ proteins, ${ }^{67-69}$ and oligonucleotides. ${ }^{70-74}$ This can be achieved through the binding of gold to sulfur-, phosphor-, nitrogen- and oxygen-based ligands, or via non-covalent interaction between capping agents. Thiol-functionalized AuNPs conjugates serve as unique probes for recognizing specific sequences in DNA segments and as building blocks for assembling novel structures and 
materials. In 1996, Mirkin and co-workers first described the use of DNA-functionalized AuNPs for DNA analysis. Thiol DNA-modified AuNPs could be well dispersed in aqueous solution to produce a red color, but when DNA linker or target was added into the solution, the AuNPs underwent network-induced aggregation, and the solution turned purple or blue (Figure 10). ${ }^{72}$ Heavy metal ions such as $\mathrm{Hg}^{2+}$ were also detected in a similar manner with excellent sensitivity and selectivity. ${ }^{73}$ Two sets of AuNPs displaying complementary DNA strands were combined in the presence of $\mathrm{Hg}^{2+}$, and formed DNA-linked aggregates due to the formation of thymidine$\mathrm{Hg}^{2+}$-thymidine complexes.
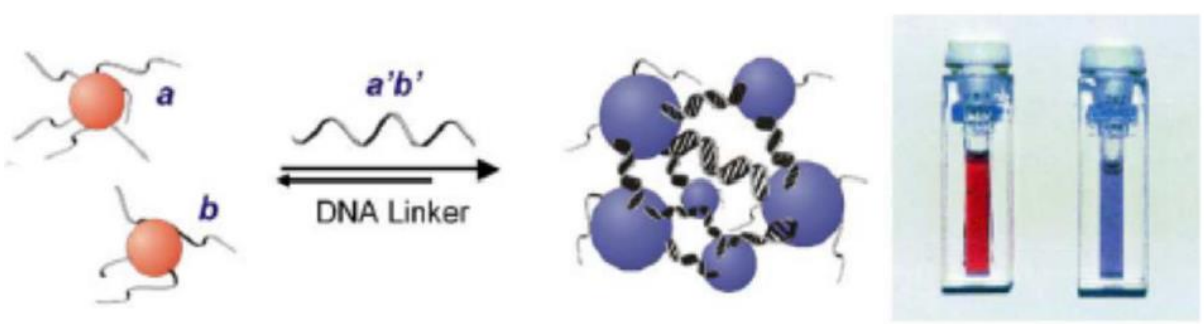

Figure 10. Schematic representation of colorimetric detection of DNA using ssDNA-modified AuNPs.

AuNPs can also undergo non-crosslinking-based aggregation to produce a red-to-blue color change. Li et al. reported the colorimetric detection of adenosine using a well-studied aptamer. The adenosine aptamer was first hybridized with a short complementary DNA attached to AuNPs, which were stable and well-dispersed in solution to produce a red color. ${ }^{74}$ Upon binding to their target, aptamer strands underwent a conformational change and dissociated from the probe-modified AuNPs. The unhybridized AuNPs now became unstable at the same salt concentration, causing immediate aggregation with a red to purple color change.

\subsection{Paper-based colorimetric sensors}

Currently, most analytical and clinical chemistry assays are performed under laboratory conditions, and require expensive instruments and trained personnel to interpret the results. Simple and portable diagnostic tests offer a good alternative for point-of-care testing, particularly 
in remote locations or developing countries. Accordingly, there is a general demand for rapid, easy-to-use and inexpensive biomedical detection assays. ${ }^{75,76}$

Paper strips for colorimetric chemical testing represent one promising solution due to several advantages relative to other assay substrate, including versatility, abundance, disposability, low cost, and ease of fabrication. Since paper is composed of a matrix of cellulose fibers and has a porous structure, it can transport liquids via capillary action without the need for an active pump or external power source. Furthermore, the surface of cellulose paper can be easily functionalized to alter its hydrophobicity, permeability and reactivity. Bleached paper also provides a bright, high-contrast background for colorimetric assays. Last but not least, paper is compatible not only with chemicals but also for the detection of different types of analytes in biological samples used for clinical diagnosis, such as urine, saliva or blood.

Recently, paper sensors functionalized with bio-recognition elements have received much attention. These elements include DNA, ${ }^{77,78}$ enzymes, ${ }^{79-81}$ and antibodies ${ }^{82,83}$ that have been deposited on the paper surface for the sensing of a wide range of chemical and biochemical species including small molecules, ${ }^{79,84}$ heavy metal ions, ${ }^{67,80,85}$ carbohydrates, ${ }^{76}$ or target molecules found on the surface of individual cells ${ }^{83}$ or even whole organisms such as fungi, bacteria, and viruses. ${ }^{82,86}$ The biorecognition elements can be delivered to the surface of these paper-based platforms by spotting, ${ }^{77}$ inkjet printing, ${ }^{80}$ vaccum filtration, ${ }^{84}$ layer-by-layer (LbL) assembly ${ }^{81}$ dip-casting, ${ }^{79}$ soaking, ${ }^{67-69,85}$ or patterned microfluidics. ${ }^{76}$

AuNPs have been widely used in paper-based sensors due to their unique electronic and optical properties. The aggregation and dispersion properties of AuNPs and the accompanying color change can be maintained on paper-based substrates. ${ }^{77,84}$ Therefore, this combination can provide opportunities for the development of cheap, portable, disposable and easy-to-use bioassays suitable for small sample volumes. Zhao et al. successfully applied an AuNP-based colorimetric paper sensor to detect deoxyribonuclease I (DNase I) activity. ${ }^{77}$ In the DNase I spot 
test, blue- or black-colored DNA-cross-linked AuNP aggregates were degraded by DNase I, producing a clear color change due to the dispersion of the nanoparticles. Luckham and Brennan ${ }^{79}$ proposed a bioactive paper "dipstick" for colorimetric detection of acetylcholinesterase (AChE) inhibitors based on sol-gel enzyme-AuNP composites. AuNPs and AChE were immobilized onto the paper and combined with sol-gel-based silica materials through dip-casting methods. AChE catalyzed the enlargement of AuNPs that were entrapped along with the enzyme in sol-gel based silica material that was coated on a functionalized paper substrate. The sensors were then allowed to cure for 24 hours at room temperature prior to use, or longer time for long-term stability studies.

\subsection{Dihydronicotinamide adenine dinucleotide, dehydrogenases, and inhibitors}

\subsubsection{NADH and its functions}

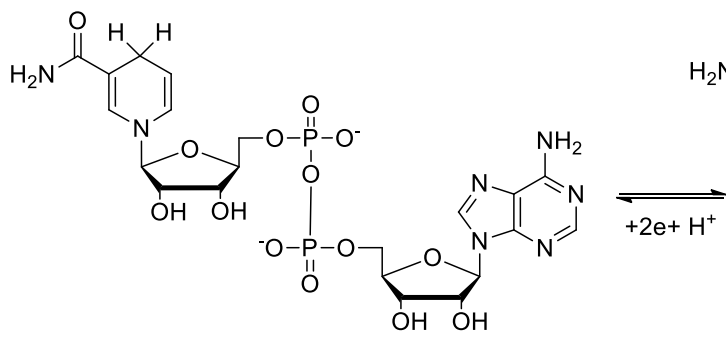

NADH

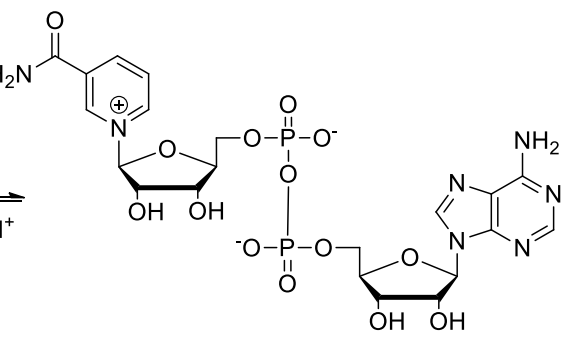

NAD ${ }^{+}$

Figure 11. Interconversion between $\mathrm{NADH}$ and its oxidized form $\left(\mathrm{NAD}^{+}\right)$.

$\mathrm{NADH}$ and its oxidized form nicotinamide adenine dinucleotide $\left(\mathrm{NAD}^{+}\right)$(Figure 11) are ubiquitous biomolecules associated with cellular energy metabolism in both eukaryotic and prokaryotic organisms. ${ }^{87}$ Recent studies indicate that these dinucleotides play a major role in numerous processes, including immunological functions, mitochondrial activity, aging and cell death. ${ }^{87-89}$ The NADH/NAD ${ }^{+}$couple transfer one proton and two electrons from one metabolite to another in many cellular redox reactions including cellular respiration and photosynthesis, and are needed to produce energy in the cells of every living organism. During this process, the nicotinamide ring of the nucleotide is oxidized or reduced (Figure 11). 
The total concentration of $\mathrm{NAD}^{+}$and NADH in most cells is $10^{-6}$ to $10^{-3} \mathrm{M}$ with a $\mathrm{NAD}^{+}$: NADH ratio ranging from 1 to $700,,^{90,91}$ which reflects the cellular metabolic status and redox state. Under oxidative stress, some cells increase their intracellular NADH/NAD ${ }^{+}$ratio or NADH levels to resist oxidative damage. ${ }^{90}$ Furthermore, the rate of NADH transformation is higher in cancer cells than in normal cells, offering a potential target for the development of cancer treatment. ${ }^{92}$ NADH deficiency leads to an energy deficit at the cellular level due to decreased ATP production, and this has been linked to multiple diseases. ${ }^{93,94}$ In the brain, NADH plays an important role in the production of vital neurotransmitters, including dopamine, norepinephrine, and epinephrine. ${ }^{95}$ Some studies have indicated that administration of NADH could increase the bioavailability of plasma levodopa and improve cognitive function, with potential benefits for patients suffering from Parkinson's disease and Alzheimer's disease. ${ }^{87,96} \mathrm{NAD}^{+}$and NADH are essential cofactors for more than 300 dehydrogenase enzymes, and the development of sensitive and specific NADH assays/sensors could open numerous possibilities for characterization of these enzymes for a variety of diagnostic, research and analytical applications. ${ }^{97,98}$

\subsubsection{Current approaches for NADH detection}

$\mathrm{NADH}$ has been detected by many different methods, including enzymatic cycling, ${ }^{99}$ colorimetry, ${ }^{22,84,100}$ fluorescence, ${ }^{101}$ chemiluminescence, ${ }^{102}$ electrochemistry, ${ }^{23,24,103,104}$ highperformance liquid chromatography (HPLC), ${ }^{105}$ and single-cell capillary electrophoresis (CE). ${ }^{90}$ The reduced and oxidized forms of these two coenzymes have distinct spectroscopic characteristics: NADH absorbs strongly around $340 \mathrm{~nm}$ and fluoresces in the blue spectral region with a maximum wavelength around $460 \mathrm{~nm}$, while its oxidized form does not. ${ }^{106}$ Measurement of NADH in a biological sample typically entails conversion to $\mathrm{NAD}^{+}$, where the fluorescence decrease at $460 \mathrm{~nm}$ is then correlated with the original NADH concentration. Similarly, NAD ${ }^{+}$ can be measured by converting it to $\mathrm{NADH}$ and recording the resulting increase in 
fluorescence. ${ }^{107}$ However, these methods are not only time-consuming but also involve cumbersome tissue-processing steps.

The enzymatic cycling assay for $\mathrm{NAD}^{+}$and $\mathrm{NADH}$ determination was first developed in the 1960s. Here, NAD ${ }^{+}$is reduced to NADH in an enzymatic reaction, while NADH is oxidized back to $\mathrm{NAD}^{+}$to produce a colorimetric or fluorescent product. ${ }^{99}$ Yeung and co-workers were able to analyze $\mathrm{NAD}^{+}$and $\mathrm{NADH}$ in a single cell using an enzymatic cycling reaction in capillary electrophoresis. ${ }^{90}$ The enzymatic reaction generated the fluorescent product resorufin, and each cycle amplified the signal through repeated reactions. The $\mathrm{NAD}^{+}$and $\mathrm{NADH}$ in the cell were separated by $\mathrm{CE}$ and allowed to undergo enzymatic reactions separately. However, the enzyme cycling assay is very complicated and requires the use of highly purified enzymes, which are susceptible to variations in detection conditions such as $\mathrm{pH}$ or redox potential.

A considerable amount of research has focused on the electrochemical oxidation of NADH over the past few decades, ${ }^{23,24,103,104}$ and the direct electrochemical oxidation of NADH has proven popular due to its fast reaction time and easy procedure. However, this reaction requires high overpotentials, which can result in interference from other easily-oxidized species present in the sample matrix. The strong adsorption of both $\mathrm{NADH}$ and $\mathrm{NAD}^{+}$can also lead to electrode surface fouling, undermining the sensitivity, reproducibility, and stability of the system.

Nanostructured materials, such as carbon nanotubes and nanoparticles, which exhibit excellent biochemical stability, good mechanical strength, and a range of electrical conductivity, also have been employed for the detection of NADH. Colorimetric detection of NADH has been reported based on anti-aggregation of AuNPs via the self-dehydration condensation of boronic acids and boronic acid-diol binding. ${ }^{100}$ One recent report described the optical detection of NADH based on AuNP growth via the strong reduction of $\mathrm{Au}^{3+}$ to $\mathrm{Au}^{0}$ by $\mathrm{NADH},{ }^{22}$ which alters the optical properties of the AuNPs. A similar assay employed reduction of $\mathrm{Cu}^{2+}$ to $\mathrm{Cu}^{0}$ by $\mathrm{NADH}$, resulting in the formation of a core-shell structure through the deposition of $\mathrm{Cu}^{0}$ on the 
AuNP surface. ${ }^{108}$ This process was imaged by taking dark-field spectra of the nanoparticles with an optical microscope. ${ }^{109}$ Although these methods provide high sensitivity; they also require sophisticated instrumentation, and are therefore ill-suited for point-of-care detection.

\subsubsection{Dehydrogenases, diseases, and inhibitors}

Biosensors for the detection of enzyme inhibitors have been developed for a broad range of analytes, such as organophosphate pesticides, organochlorine pesticides, and heavy metals. ${ }^{110}$ Inhibitors have been identified for a large number of enzymes, such as urease, invertase, xanthine oxidase, peroxidase, glucose oxidase and alkaline phosphatase. ${ }^{110}$ For biosensors based on enzyme inhibition, enzyme activity is measured both before and after exposure of the biosensor to the analyte of interest, and the residual enzyme activity is inversely related to the inhibitor concentration. Inhibition is calculated using Equation 1: ${ }^{111}$

$$
I \%=\frac{A_{0}-A_{i}}{A_{0}}
$$

where $\mathrm{I} \%=$ percentage of inhibition; $\mathrm{A}_{0}=$ the enzyme activity before exposure to the analyte; and $A_{i}=$ the enzyme activity after exposure to the analyte.

Dehydrogenase inhibitors have been established for the treatment of human diseases, ${ }^{112-114}$ as well as applications in alcohol dependence, ${ }^{115}$ cocaine addiction, ${ }^{116}$ anxiety, ${ }^{117}$ and as resensitizing agents for cancers. ${ }^{118}$ Dehydrogenase inhibitor discovery is also crucial for the development of novel anticancer agents, ${ }^{119}$ antibiotics, ${ }^{120}$ and pesticides. ${ }^{121}$ For example, lactate dehydrogenase (LDH) is an enzyme utilizes NADH as a cofactor to catalyze the conversion of pyruvate to lactate in the glycolysis pathway. LDH is overexpressed in cancer cells, ${ }^{122}$ which rely heavily on anaerobic glycolysis to generate ATP. Inhibition of LDH with siRNA or small molecules has been reported to induce oxidative stress and cell death. ${ }^{122}$ Inhibition of this enzyme depletes the cellular energy supply, which reduces the metastatic and invasive potential of cancer cells. ${ }^{123}$ LDH therefore offers a potential therapeutic target for cancer metabolism. 
Inosine 5'-monophosphate dehydrogenase (IMPDH), which catalyzes the oxidation of inosine 5-monophosphate (IMP) to xanthosine $5^{\prime}$-monophosphate (XMP) while reducing $\mathrm{NAD}^{+}$to $\mathrm{NADH}$, represents another potential anticancer target. IMPDH is required for the biosynthesis of guanosine monophosphate (GMP); inhibition of the enzyme causes an overall reduction in guanine nucleotide pools, which subsequently hinders DNA and RNA synthesis and results in cytotoxicity. In addition to cancer, IMPDH is an attractive target for the development of antiviral and antiparasitic drugs, as well as immunosuppressive chemotherapy. ${ }^{124}$

Aldehyde dehydrogenases (ALDH) are important in alcohol metabolism, oxidizing toxic aldehydes into carboxylic acids through an NADH-dependent reaction. Increased activity of ALDH has been reported in various cancers, ${ }^{114}$ and has been found to interfere with certain chemotherapeutic treatments. ${ }^{112,125}$ ALDH dysfunction is also believed to be a contributing factor for a variety of other human diseases, including diabetes, stroke, cardiovascular and neurodegenerative diseases. Selective inhibition of ALDH may have therapeutic potential for some of these conditions, and could also help in treating human alcohol abuse by increasing acetaldehyde accumulation in the body ${ }^{115}$ and cocaine addiction by decreasing cocaine-stimulated dopamine production and release. ${ }^{116}$

For this dissertation, glucose dehydrogenase $(\mathrm{GDH})$ was chosen as a model enzyme to evaluate the performance and generalizability of a paper-based colorimetric device for monitoring $\mathrm{NAD}^{+}$-driven biochemical process in the presence or absence of inhibitors. Glucose dehydrogenase $(\mathrm{GDH})$ catalyzes the oxidation of $\beta$-D-glucose to gluconic acid while reducing $\mathrm{NAD}^{+}$to $\mathrm{NADH}$; the concentration of glucose therefore determines the amount of NADH produced. ${ }^{126}$ Heavy metal ions are known to negatively affect the survival of plants and animals even at very low concentrations, and both $\mathrm{Ag}(\mathrm{I})$ and $\mathrm{Hg}(\mathrm{II})$ are known to act as strong inhibitors of GDH; no such inhibitory effect has been reported for $\mathrm{Pb}(\mathrm{II}){ }^{127}$ This colorimetric assay platform offers the ability to rapidly and sensitively screen GDH inhibitors based on their 
modulation of the NADH production by the naked eye, and this platform design is most likely generalizable to hundreds of different dehydrogenase enzymes.

\subsection{Single nucleotide polymorphisms (SNP) and disease}

\subsubsection{Introduction to SNPs}

Many human diseases can be directly traced back to sequence mutations within the genome, ${ }^{128}$ and the identification of genetic mutations is very important for both early diagnosis and the selection of treatment regimens. If there is a family history of a genetic condition, genetic testing can indicate markers of risk long before symptoms manifest. Newborn genetic screening is also commonly performed in the United States to screen for the certain genetic abnormalities. For example, if testing indicates strong risk for a disorder such as phenylketonuria (PKU) or congenital hypothyroidism, appropriate treatment can be applied right away.

Single nucleotide polymorphisms (SNPs) account for $90 \%$ of human genetic variability. ${ }^{129}$ Each SNP represents a change in single nucleotide from one base (A, T, C, or G) to another, and SNPs occur in the genome at a rate of approximately one per every 1,000 bases. On average, $\sim 500,000$ SNPs reside in the coding regions of the human genome, equivalent to an average of $\sim 6$ per gene. Ninety percent of human genetic variation takes the form of SNPs, and these are involved in the etiology of many human diseases. ${ }^{130}$ These genetic variations are directly related to patients' individual responses to diseases, drugs, and other environmental factors. Multiple SNPs have found linked to Alzheimer's, ${ }^{131}$ Parkinson's, ${ }^{132}$ diabetes, ${ }^{133}$ and various cancers. ${ }^{134}$ SNP detection is therefore important for early diagnosis, clinical prognostics, and disease prevention. $^{135,136}$

Many strategies have been developed to detect SNPs. Historically, mutations have been detected by Sanger sequencing in the laboratory. This technique employs DNA synthesis by multiple parallel reactions with DNA polymerase in which the various deoxynucleotide triphosphates (dNTPs) are also combined with small quantities of dideoxynucleotide 
triphosphates (ddNTPs), which terminate the DNA synthesis reaction. Sanger sequencing is the gold standard technique for DNA analysis, with a $100 \%$ detection rate. However, it is also laborious, technically demanding, costly and time-consuming.

Enzyme-based SNP detection methods include microarray-based sequencing, ${ }^{137,138}$ primer extension, ${ }^{139}$ ligation-based methods, ${ }^{140}$ enzyme invasive cleavage ${ }^{141}$ and TaqMan assays. ${ }^{142}$ These methods can achieve parallel, sensitive detection of SNPs, but they are also complex, multi-step techniques that require costly modified primers and are often plagued by issues related to enzymatic efficiency and product separation. Hybridization-based SNP detection method such as dynamic allele-specific hybridization, ${ }^{143}$ molecular beacons, ${ }^{144}$ binary probes, ${ }^{145,146}$ and triplestem probes ${ }^{147}$ offer an alternative for identifying specific target sequences. However, these suffer from low or poor specificity at room temperature, and effective SNP discrimination requires the use of high-stringency conditions with precise temperature control.

Besides fluorescence-based detection, mass spectroscopy, ${ }^{148}$ electrochemistry, ${ }^{149}$ and gel electrophoresis ${ }^{150}$ also have been used as signal reporters for SNP detection. In general, those methods are incompatible with high-throughput analysis, and impractical for use in a small hospital or developing-world setting. As such, there remains an urgent need for a simple platform for rapid and sensitive SNP detection at room temperature. Colorimetric methods could fulfill this need, since they enable cost-effective naked-eye detection without sophisticated equipment.

\subsubsection{AuNP-based methods for SNP detection}

AuNPs have been used as a colorimetric reporter for SNP sensing based on their unique SPR absorption properties, ${ }^{64,70}$ where AuNP aggregation or redispersion of an aggregate triggers a visible color change. As described above, electrostatic interactions between ssDNA and citratecoated AuNPs stabilizes the AuNPs against aggregation at high salt conditions, but this does not occur with dsDNA. ${ }^{64}$ One study demonstrated that SNPs can be detected based on the color change of unmodified AuNPs within 5 minutes, which is based on the differences in electrostatic 
interaction between single- and double-stranded DNA with unmodified AuNPs. ${ }^{64}$ Typically, the single-stranded DNA adsorbed on the AuNPs stabilizes the nanoparticles against aggregation at salt concentrations that would ordinarily cause colloid aggregation. More recently, Liu proposed an approach for discrimination of single-base mismatches through probe-target hybridization followed by treatment with structure-selective $\mathrm{S} 1$ nucleases. ${ }^{29} \mathrm{~S} 1$ nuclease is inactive on dsDNA duplexes, but capable of degrading single-stranded DNA. ${ }^{151}$ When a perfectly matched target hybridized to the probe sequence, the resulting duplexes were protected from S1 nuclease. In contrast, non-complementary/mismatched targets containing a SNP were digested to release dNMPs, which are even more effective at stabilizing AuNPs against salt-induced aggregation than ssDNA. ${ }^{29}$ The main drawback of this system is limited sensitivity.

DNA-conjugated AuNPs have gained popularity as one of the most fascinating nanomaterials due to their unique chemical and physical properties. ${ }^{72,152,153}$ The first thiolated DNA-modified AuNPs-based platform for SNP detection was reported by Mirkin and coworkers, which relied on hybridization-driven changes in AuNP aggregation. ${ }^{5}$ In this assay, two sets of DNA-modified AuNPs were cross-linked via target hybridization, and a single-base mismatch could be directly observed on a $\mathrm{C} 18$ thin-layer chromatography plate at $58{ }^{\circ} \mathrm{C} .{ }^{5}$ Wang and coworkers explored toehold-mediated stand-displacement reactions with AuNP fluorescence anisotropy signal enhancement in the development of a homogeneous SNP detection assay. ${ }^{154}$ Here, fluorescently labeled DNA strands are detached during target hybridization, resulting in low fluorescence anisotropy, and differences in dynamics and kinetics can distinguish SNP-containing and perfectly-matched target strands. SNP detection has also been performed with hybridizationmediated growth of AuNP probes. This method can be employed not only for the semiquantitative analysis of the target DNA. but also for the identification of base-pair mismatches (both single and multi-nucleotide polymorphisms) in DNA samples. ${ }^{155}$ However, even for welloptimized conditions, reliable detection with unmodified AuNPs required a target concentration 
higher than $200 \mathrm{nM}$ for naked eye observation. ${ }^{156}$ To obtain clinically relevant sensitivity, this and subsequently-developed AuNP-based SNP detection platforms have employed polymerase chain reaction (PCR), ${ }^{157}$ nicking endonuclease, ${ }^{158}$ rolling circle amplification ${ }^{159}$ or real-time ligation chain reaction ${ }^{160}$ to amplify the target prior to detection. Low picomolar concentrations of the target can be successfully detected using these amplified approaches, but the target-triggered aggregation of AuNPs still requires a reaction time of at least two hours under precisely controlled temperature.

\subsection{DNA surface coverage on AuNPs}

DNA-conjugated AuNPs can serve as unique probes for recognizing specific sequences in DNA segments and as building blocks that can be assembled together. These applications depend on the hybridization of the AuNP-conjugated DNA to the target DNA in solution. Two factors determine the sensor performance: hybridization efficiency of the surface-bound probe, and the number of targets captured. ${ }^{161}$ The probe density or surface coverage of the ssDNA on the gold surface determines the DNA orientation at the interface, and thus the efficiency of the hybridization process. However, maximizing the surface coverage may not yield the best conditions for hybridization efficiency, since high surface density of the probe can lead to increased steric and electrostatic repulsion between the DNA probe and target, resulting in low hybridization efficiency and a longer reaction time. ${ }^{162,163}$ In addition, tight packing of the DNA on the gold nanoparticle surface can impede degradation by nucleases. ${ }^{164}$ At low surface density, there is almost no steric hindrance between the probe and target, and almost $100 \%$ of probes can be hybridized. ${ }^{165}$ However, these DNAs are more likely to lie on the surface due to strong interactions between the ssDNA and the gold substrate. Furthermore, surface coverage of the DNA significantly affects the thermal and chemical stability of DNA-AuNP conjugates, ${ }^{164,} 166$ sensor sensitivity, ${ }^{167}$ and DNAzyme activity. ${ }^{168}$ Therefore, optimization of probe density is necessary to balance good hybridization efficiency with a fast reaction time. 
The loading of DNA on Au substrate can be controlled during the modification step by varying ionic strength, base composition, DNA concentration, co-adsorption with short hydroxyterminated alkanethiol diluent molecules, or assistance with sonication and electrically-induced desorption. ${ }^{71,162,164,167,169-172}$ The ionic strength of the solution in which the probes are immobilized greatly affects the surface density of ssDNA on the gold substrate due to the effect of electrostatic repulsions between DNA strands. Published protocols for loading alkanethiol-capped DNA onto gold substrate have tested a variety of salt ${ }^{162,163,169,171}$ (Figure 12) or buffer concentrations ${ }^{161,173}$ to minimize these electrostatic effects. When the solution ionic strength is high, the electrostatic repulsion between negatively charged DNA molecules are effectively screened. Thus, higher probe coverage can be obtained, whereas low ionic strength solutions resulted in low surface density.

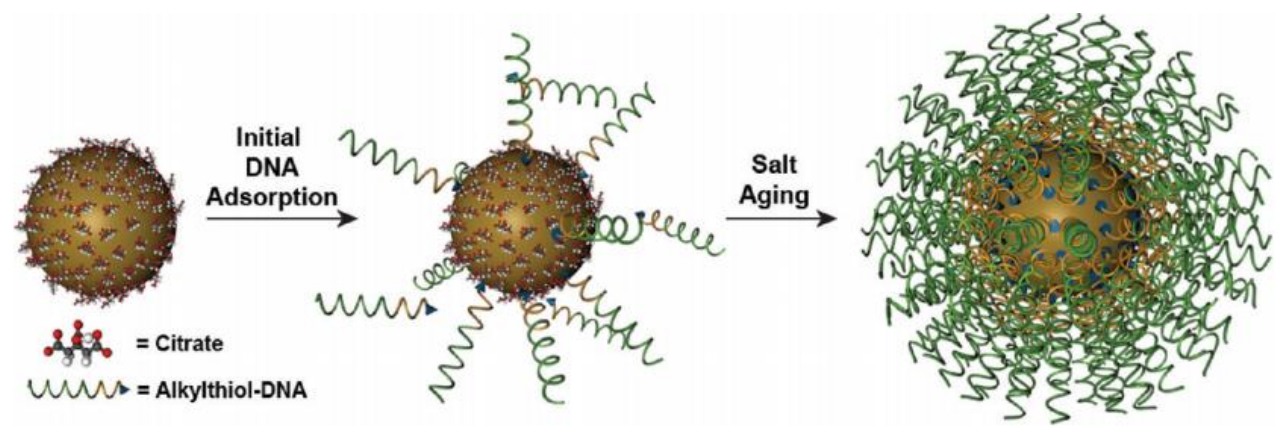

Figure 12. Synthesis of thiolate DNA-AuNP conjugates.

The DNA loading is dependent on oligonucleotide base sequence. The covalent conjugation of thiolated DNA onto the gold surface is most commonly achieved by thiol-gold chemistry. ${ }^{5}$ With this method, a thiol modification at the $5^{\prime}$ or $3^{\prime}$ end of the oligo enables formation of a selfassembled monolayer (SAM) on the gold surface. However, DNA bases can also interact with gold surface, ${ }^{174}$ causing nonspecific adsorption of DNA and reducing the target DNA hybridization efficiency. Thymine has a much lower binding affinity for gold surfaces than other deoxynucleotides, ${ }^{175}$ and simple repeats of thymine bases (poly-T spacer) are frequently added to 
thiolated DNA probes to prevent nonspecific adsorption. The length of the polyT spacer has been explored $^{168}$ to increase DNA surface coverage on AuNPs. Additionally, poly-A spacers have also been used to adjust the surface density of non-thiolated DNA based on its high affinity for gold surfaces, and surface density decreased as the length of the spacer increased. ${ }^{176}$

Another way to control surface density is to simply adjust the DNA concentration employed for the modification. High concentrations of thiolated DNA often produce high surface coverage, and vice versa. However, a low DNA surface coverage results in non-specific adsorption of DNA on the gold surface, which greatly inhibits DNA hybridization efficiency. To achieve hHigher hybridization efficiency between the target DNA and probe-modified AuNPs can be achieved through co-adsorption of the ssDNA and a thiol-molecule such as mercaptohexanol $(\mathrm{MCH})$ to form a mixed-SAM on the gold surface. ${ }^{163,177,178}$ This mixed-SAM forces the DNA strands away from the gold surface, and therefore allows greater DNA loading on the surface.

These simple, single-step assembly procedures allow for better control of the DNA surface density, but also have their own drawbacks. For example, several modification samples and careful experiment design are required to obtain different surface coverages; for example, excess salt might lead to irreversible aggregation of AuNPs during the modification step. In addition, the surface coverage of DNA can only be tuned within a limited range, and it becomes complicated when precise control of DNA density is required.

A two-step method has been reported to precisely control the surface coverage on a gold substrate. After DNA modification, surface density can be decreased via ligand-exchange reaction by using "backfilling" molecules, thiol molecules that are shorter than the DNA probes. ${ }^{163,171,173,179,180}$ Specifically, the gold substrate is exposed to thiolated ssDNA solution first, followed by exposure to a backfilling molecule solution, which form a mixed monolayer of DNA and thiolated molecules. This ligand-exchange process removes both non-specifically adsorbed DNA and some of the covalently attached DNA from the surface, depending on the exposure time 
in diluent solution or the concentration of backfilling molecules. 6-mercapto-1-hexanol (MCH), a six-carbon chain and water-soluble thiol molecule with a hydrophilic hydroxyl group, has often been used as a backfilling molecule to regulate the number of DNA molecules on gold surfaces due to its high affinities for gold. $\mathrm{MCH}$ can reduce nonspecific DNA binding to the gold surface and rearrange the conformational change of oligonucleotide, ${ }^{179}$ thereby improving DNA probe orientation and target hybridization efficiency.

This backfilling approach can enhance sensor performance by modulating the salt tolerance of DNA-conjugated AuNPs. ${ }^{74,161,177,180}$ For example, in one study, an adenosine-binding aptamer was hybridized with a short complementary AuNP-conjugated DNA, which was stable and well dispersed in the solution. The aptamer strands underwent structure-switching upon binding adenosine, leading to their release from the AuNPs. ${ }^{74}$ The ssDNA-conjugated AuNPs now became unstable and aggregated to produce a rapid red-to-purple color change. However, when these AuNPs have high surface coverage (150 strands/AuNP), DNA-modified AuNPs are found highly stable even in the presence of $500 \mathrm{mM} \mathrm{MgCl}$, and the aptamers could not function well under such high divalent metal ion concentrations. ${ }^{74} \mathrm{MCH}$ molecules were therefore added to the solution to decrease DNA surface coverage to 100 DNA strands/AuNP, and successful target detection became possible at this reduced surface coverage. ${ }^{74}$ Song and Liu reported colorimetric detection of DNA methylation using DNA-AuNPs in a restriction endonuclease-based assay. ${ }^{180}$ They treated the DNA-AuNPs with MCH for 8 minutes to decrease the surface density from $\sim 90$ to $\sim 35$ DNA strands/AuNP, which greatly increased the digestion activity of restriction endonuclease. In addition to $\mathrm{MCH}$, oligo(ethylene glycol) (OEG)-terminated alkanethiols ${ }^{161,177}$ has also been used as a diluent to regulate DNA coverage on gold surfaces and reduce nonspecific interactions with proteins and other biomolecules. Dithiothreitol (DTT), can also be attached to gold surfaces via two Au-S bonds while retaining its two hydroxyl groups exposed at the outer surface, ${ }^{181}$ thus providing a hydrophilic environment that is favorable for DNA target 
hybridization. Recently, a tertiary SAM structure was described, incorporating a thiolated capture probe as well as MCH and DTT as a means to improve DNA detection. ${ }^{182}$

\subsection{Cocaine and cocaine-binding aptamer}

\subsubsection{Cocaine abuse}

Cocaine (Figure 13) is a powerful, psychoactive and addictive central nervous system (CNS) stimulant that is classified as a Schedule II drug. ${ }^{184}$ Cocaine remains the leading cause of emergency hospital visits and illicit drug-related deaths-including from causes such as AIDS, homicide, suicide, and accidents - in the United States. The 2014 National Survey on Drug Use and Health (NSDUH) reported that about 1.5 million people aged 12 or older were current users of cocaine. ${ }^{185}$ Cocaine increases the level of dopamine, a neurotransmitter associated with pleasure and movement. Cocaine abuse leads to adverse health consequences including anxiety, paranoia, organ damage, violent behavior, increased risk of HIV/AIDS, and even death. ${ }^{186}$ Exposure to cocaine during pregnancy is associated with low birth weight, early gestational age, premature delivery, and birth defects. ${ }^{187}$

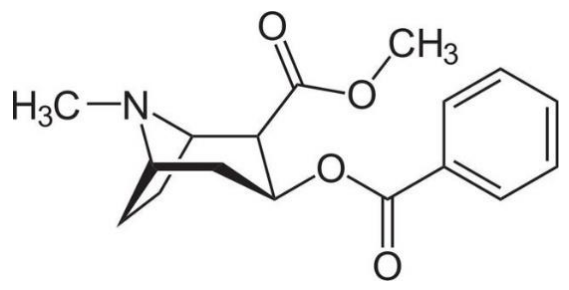

Figure 13. Chemical structure of cocaine.

\subsubsection{Current methods for cocaine detection}

A number of methods of varying sensitivity and specificity have been employed for qualitative and quantitative analysis of cocaine or its metabolites. Usually, detection is achieved with a presumptive screening test, followed by a confirmatory test. ${ }^{188,189}$ Immunoassays are among the most common methods for initial presumptive screening, as they allow rapid screening of many specimens and can confidently exclude drug-negative samples from further confirmatory 
testing. Commercially-available tests such as enzyme-linked immunosorbent assay (ELISA), ${ }^{190,}$ 191 enzyme-multiplied immunoassay technique (EMIT), ${ }^{192}$ fluorescence polarization immunoassay (FPIA) ${ }^{193}$ and radioimmunoassay (RIA) ${ }^{189}$ can achieve quantitative and qualitative detection of cocaine and/or its major metabolites in biofluids. ELISA has been proven to be time consuming and expensive. EMIT is the most commonly-used and cheapest assay for simple, rapid screening, but its sensitivity is low. ${ }^{194}$ RIA achieves very sensitive detection, but the reagents are expensive and special technical skills are required. RIA also involves procedures for handling radioactive waste. ${ }^{189}$ Furthermore, antibody-based screening tests often cannot distinguish between drugs and structurally-similar legal substances, resulting in false positives. ${ }^{195,196}$

Confirmatory tests for cocaine detection are based on mass spectrometry. ${ }^{188,189,197}$ Gas chromatography/mass spectrometry (GC/MS) is considered a "gold standard" for the toxicological detection of cocaine. ${ }^{195,198}$ Here, samples are first separated by creating a partition between mobile (e.g., liquid or gas) and stationary phases, and then introduced into the mass spectrometer and the isolated molecules are characterized based on their mass-to-charge ratio. The corresponding spectra provide a unique fingerprint for each moiety of the drug molecule. GC/MS detection of cocaine is highly sensitive, reliable and extremely specific. However, the instruments are expensive and trained personnel are essential to operate the instruments and interpret the results. Thus, a more accurate on-site screening analysis is still needed to achieve simple and rapid drug testing with high specificity and sensitivity.

\subsubsection{Development of aptamers}

Recently, aptamer-based biosensors have been introduced as new alternative methods to perform detection of small molecules. ${ }^{199}$ In 1990, several researchers used an in vitro selection technique, termed systematic evolution of ligands by exponential enrichment (SELEX), ${ }^{200}$ to isolate synthetic RNA motifs that bound specifically to T4 DNA polymerase. These specific oligonucleotide sequences, which the researchers named aptamers, ${ }^{201}$ usually have a molecular 
weight of 5 - $40 \mathrm{kDa}$ and can fold into three-dimensional structures via hydrogen bonding, electrostatic interactions and/or van der Waals forces. Aptamers usually display high affinity for their targets with good specificity, and some protein-binding aptamers have dissociation constants in picomolar range. ${ }^{202}$ Aptamers have demonstrated excellent selectivity in discriminating targets from interferent molecules with only slightly differing structures, such as the presence or absence of a methyl group ${ }^{203}$ or structural enantiomers. ${ }^{204}$ For example, the arginine-binding aptamer has a 12,000-fold better selectivity for L-arginine than for D-arginine. ${ }^{204}$

Aptamers have the potential to surpass antibodies as affinity reagents due to several advantages. First, aptamers can be isolated against almost any target, including proteins, ${ }^{205}$ viruses,${ }^{206,207}$ cell-surface markers, ${ }^{208-211}$ small molecules, ${ }^{78,212,213}$ and metal ions. ${ }^{27,214}$ In contrast, it can be difficult to generate antibodies against non-immunogenic targets such as ions and small molecules. ${ }^{215}$ Second, aptamers are selected in vitro under non-physiological conditions, whereas antibodies are produced in vivo under physiological conditions. ${ }^{200}$ Therefore, aptamers can easily be generated for targets such as toxins, carcinogens or small-molecule drugs. ${ }^{216}$ Third, once aptamers are selected, they can be rapidly synthesized or chemically modified within a few days, rather than requiring the protracted biological methods used to produce antibodies. Fourth, the accuracy and reproducibility of DNA and RNA synthesis allows for relative ease in producing aptamers at large scales, with very little batch-to-batch variation. Finally, the stability and bioavailability of aptamer can be easily improved by post modification. ${ }^{215}$

Aptamers have been widely used for diagnosing disease, ${ }^{205,210,211,217}$ drug delivery ${ }^{218}$ and therapeutics. ${ }^{218-222}$ Some therapeutic aptamers have progressed through preclinical to clinical development. ${ }^{223}$ The most successful example of these is pegaptanib sodium (brand name Macugen, Eyetech Pharmaceuticals/Pfizer), which was approved by the US Food and Drug Administration (FDA) in 2004 for the treatment of neovascular (wet) age-related macular degeneration (AMD) caused by the growth of abnormal, leaky blood vessels. ${ }^{219-221}$ Wet AMD, a 
retinal disease that causes severe and irreversible vision loss by damaging the area of the eye responsible for central vision, is one of the main reasons for blindness for individuals over $55 .{ }^{224}$ When injected into the affected eye, the aptamer selectively binds to the vascular endothelial growth factor isoform $\mathrm{VEGF}_{165}$ and inhibits the growth of blood vessels and prevents vascular leakage. Pegaptanib is the first aptamer to be used as a therapeutic agent in humans, and represents a milestone in drug development.

\subsubsection{Aptamer-based biosensors for cocaine detection}

Aptamers have been widely used for sensing and diagnostic applications. ${ }^{200,}{ }^{201}$ MNS-4.1, the first cocaine-binding aptamer, which was isolated by Stojanovic group, has been closely studied for the development of biosensors due to its high specificity and selectively towards cocaine over its major metabolites. ${ }^{213}$ Stojanovic et al. developed a fluorescence-based sensor for cocaine detection using a fluorophore-quencher-modified cocaine-binding aptamer based on MNS-4.1. ${ }^{213}$ In the absence of cocaine, the unfolded aptamer fluoresces, with minimal quenching of the fluorophore. In the presence of cocaine, the aptamer forms a noncanonical three-way junction with a hydrophobic pocket (Figure 14), which brings the fluorophore and quencher and leads to $\sim 50 \%$ quenching of fluorescence signal. This assay was able to detect $10 \mu \mathrm{M}$ cocaine in serum samples. ${ }^{213}$ Since then, various transducers have been coupled with cocaine-binding aptamers, including fluorescence, ${ }^{212,213,225-227}$ colorimetry, ${ }^{228-230}$ chemiluminescence, ${ }^{231,232}$ electrochemistry, ${ }^{233-235}$ and surface-enhanced Raman scattering. ${ }^{236}$ One colorimetric sensing platforms using a Cy7 cyanine dye displacement strategy was reported by Stojanovic's group. ${ }^{229}$ Specifically, the addition of cocaine to preformed aptamer-Cy7 dye complexes resulted in the displacement of the dye, causing a decrease of absorbance that was proportional to the cocaine concentration. This target-displacement-based strategy requires specific dyes that not only bind to the aptamer efficiently but also can be subsequently displaced by target binding. Unfortunately, few dyes meet both requirements, hindering the further development of such assays. 


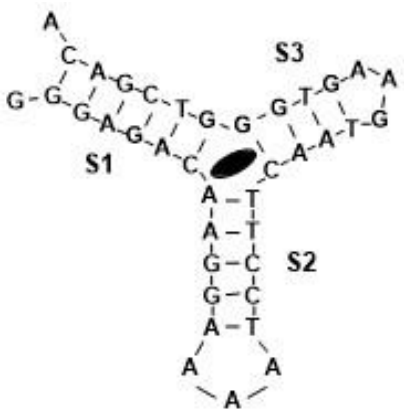

MNS-4.1

Figure 14. Three-way junction structure of the cocaine-bound MNS-4.1 aptamer.

This cocaine-binding aptamer undergoes a target-induced conformational change. However, in practice its conformation exists in equilibrium between the folded and unfolded structures, resulting in high background signal and limited sensitivity. ${ }^{213}$ In order to decrease background signal, cocaine-binding aptamers have been split into two ${ }^{212}$ or three fragments. ${ }^{237,238}$ The resulting split aptamer fragments were found to retain good specificity for their targets. Most importantly, the fragments do not interact with one another in the absence of cocaine. Stojanovic's group was the first to report fluorescent detection of cocaine with a split aptamer. ${ }^{212}$ The two fragments were tagged with a fluorophore and quencher respectively, in the presence of cocaine, the two aptamer fragments were brought together by the formation of a target-induced assembly and the fluorescence intensity was significantly decreased. However, this still yielded a poor detection limit, because the splitting of the aptamer resulted in reduced target affinity $\left(\mathrm{K}_{\mathrm{D}}\right.$ $\sim 200 \mu \mathrm{M}){ }^{212}$ Plaxco's group used a split cocaine-binding aptamer to develop an electrochemical sensor in which one of the unmodified fragments was attached to a gold electrode surface, whereas the other remained free in solution and was modified with the redox label methylene blue. ${ }^{239}$ The addition of cocaine promoted the formation of aptamer-cocaine tertiary structures on the electrode surface, bringing the redox label in close proximity to the electrode surface and generating a large faradaic current. Willner's group reported fluorescence detection of cocaine by labeling aptamer fragments with semiconductor quantum dots, dyes or pyrene units. ${ }^{240}$ They 
found that the formation of aptamer-substrate complexes resulted in fluorescence resonance energy transfer (FRET) or pyrene excimer emission. They also developed sensor platforms to perform cocaine detection using different modified aptamers. ${ }^{241}$ Specifically, several transducers were demonstrated by using $\mathrm{Pt}$ nanoparticles for electrocatalytic reduction of $\mathrm{H}_{2} \mathrm{O}_{2}, \mathrm{CdS}$ semiconductor nanoparticles in the presence of triethanolamine for photoelectrochemical detection, and AuNPs modified Au substrate for SPR. Willner also developed an exonuclease III (Exo III)-based aptasensor for the amplified detection of cocaine ${ }^{242}$ using split aptamer consisting of one unmodified fragment and one fluorophore/quencher-modified fragment. In the absence of cocaine, the fragments remain in a single-stranded state that cannot be digested by Exo III, and no fluorescence is observed. Cocaine triggered assembly of these two fragments into a duplexed target-aptamer complex that can be recognized and digested by Exo III. This results in release of the quencher, producing a strong fluorescence signal. This releases both the target and the unmodified fragment, which allows them to be recycled for further amplification of the fluorescence signal, resulting in a detection limit of cocaine of $50 \mathrm{nM}$.

Colorimetric sensors have significant advantages for on-site and point-of-care detections because these sensing platforms can minimize or even eliminate the requirement of analytical instruments and made naked-eye observation highly achievable. The colorimetric detection of cocaine has been reported based on the catalytic activity of the peroxidase-mimicking Gquadruplex DNAzyme. ${ }^{243}$ One of the aptamer fragments was modified onto amine-functionalized magnetic nanoparticles (MNPs), while the other remained in solution. Cocaine binding promoted assembly of the two fragments onto the MNP, forming a three-way junction on the surface. The G-quadruplex DNAzyme then catalyzed a TMB- $\mathrm{H}_{2} \mathrm{O}_{2}$ reaction, producing a visible color change. This system achieved a detection limit of $50 \mathrm{nM}$. AuNPs have also been used as a colorimetric probe for cocaine detection. Fan et al. developed a simple colorimetric assay for cocaine detection using unmodified AuNPs and aptamer fragments in solution. ${ }^{244}$ In the absence of 
cocaine, the AuNPs remained red because non-specific adsorption of the aptamer fragments onto the AuNP surface stabilized the particles against salt-induced aggregation. In the presence of cocaine, the formation of aptamer-cocaine tertiary structure resulted in AuNP aggregation and produced a blue color. ${ }^{244}$ This assay achieved a detection limit as low as $2 \mu \mathrm{M}$ cocaine. However, high concentrations of cocaine tended to induce the aggregation of unmodified AuNPs, producing a false positive signal. Another assay based on the cocaine-induced disassembly of aptamerlinked AuNP aggregates has also been developed for the colorimetric detection of cocaine. ${ }^{228}$ Upon addition of cocaine, the aptamer underwent a target-induced conformational change, dissociating from the AuNP surface and thereby causing disruption of the AuNP aggregates. 


\section{CHAPTER 3: AuNP-based chemical sensor: A paper-based device for rapid visualization of NADH based on dissolution of gold nanoparticles}

\subsection{Introduction}

AuNPs have been widely used for DNA and small molecules detection because of their unique distance- and size-dependent surface plasmon resonance (SPR) absorption. ${ }^{3-6}$ The size of AuNPs can be easily controlled either during or after their synthesis. Specifically, the enlargement of AuNPs can be achieved by silver enhancement, ${ }^{245}$ or seed-mediated growth under mild reducing conditions in a growth solution which contains gold precursor and surfactant. ${ }^{22}$ The size of AuNPs can also be easily decreased via chemical reactions in the presence of ligand/oxidant including $\mathrm{CN}^{-} / \mathrm{O}_{2},{ }^{43-45} \mathrm{I}_{2} / \mathrm{I}^{-}$or $\mathrm{I}_{2} / \mathrm{SCN}^{-},{ }^{47} \mathrm{Cl}^{-} / \mathrm{H}_{2} \mathrm{O}_{2}{ }^{48}$ in an aqueous or non-aqueous medium. ${ }^{49,50}$ Recently, mild oxidation of AuNPs has been obtained using the solutions containing a surfactant such as CTAB. ${ }^{52-54}$ Aguirre et al. first observed that exposure to CTAB in aqueous solutions enabled reshaping of gold shells to form highly asymmetric gold rod- or bean-like structures at room temperature. ${ }^{14}$ Rodriguez-Fernandez et al. subsequently reported the sculpting of $\mathrm{Au}$ nanospheres via oxidation of AuNPs in $\mathrm{Au}^{3+}-\mathrm{CTAB}$ solution, and proposed that the charged CTAB micelles spatially directed the oxidation of nanoparticles. ${ }^{53}$ Other groups have applied the dissolution for the resizing or reshaping of gold AuNRs. ${ }^{50,55}$ More recently, many colorimetric sensors for the detection of $\mathrm{Fe}^{3+}, \mathrm{Cu}^{2+}, \mathrm{Cr}^{6+}, \mathrm{Pb}^{2+}, \mathrm{H}_{2} \mathrm{O}_{2}, \mathrm{HCl}, \mathrm{NO}_{2}{ }^{-}$and $\mathrm{I}^{-}$have been reported based on the oxidative etching of AuNPs or AuNRs. ${ }^{48,51,56-62}$ For example, a colorimetric sensor based on the selective etching of AuNRs for the detection of $\mathrm{Cr}^{6+}$ was developed. The $\mathrm{Cr}^{6+}$ ions perform strong oxidization towards AuNRs to decrease the aspect ratio of AuNRs, generating a blue shift in SPR absorption and a visible color change from bluish-green or red to light red and then to colorless as the target concentration increased. ${ }^{59}$ However, incubation for more than 5 hours at high temperature $\left(50-70{ }^{\circ} \mathrm{C}\right)$ is required to observe the color change, and so far only inorganic targets have been used with these sensor platforms. 
$\mathrm{NADH} / \mathrm{NAD}^{+}$are ubiquitous biomolecules associated with cellular energy metabolism in both eukaryotic and prokaryotic organisms. ${ }^{87}$ Recent studies indicated that these dinucleotides have play an major role in numerous processes, including immunological functions, mitochondrial activity, aging and cell death. ${ }^{87-89}$ The investigations of the redox reactivity of $\mathrm{NADH}$ and $\mathrm{NAD}^{+}$are important because a large number of dehydrogenase enzymes $(>300)$ use these compounds as cofactors. Thus, the development of sensitive and specific NADH sensors ${ }^{97}$, ${ }^{98}$ could open numerous possibilities for dehydrogenase characterization as well as for screening to identify inhibitors of dehydrogenases for the development of novel anticancer agents, ${ }^{119}$ antibiotics, ${ }^{120}$ and pesticides. ${ }^{121}$

We recently discovered that NADH could inhibit the dissolution of AuNPs at room temperature. ${ }^{84}$ Thus, we believe that NADH-mediated inhibition of AuNP dissolution could enable simple and direct detection of dehydrogenase-driven $\mathrm{NAD}^{+}$-reduction for a number of analytes. In this chapter, we reported an AuNP-based chemical sensor on a paper-based device that enabled rapid and sensitive room-temperature detection of NADH via AuNP dissolution related colorimetric readout, and demonstrated its value for rapid screening dehydrogenase inhibitors by monitoring $\mathrm{NAD}^{+}$-driven enzymatic reactions.

To achieve this goal, we first proved a sensitive dissolution of AuNPs in the $\mathrm{Au}^{3+}-\mathrm{CTAB}$ solution, and demonstrated a successful NADH-mediated inhibition of AuNP dissolution with UV-Vis spectrometry. To develop an instrument-free detection platform, the citrate-capped AuNPs were deposited onto a mixed cellulose ester (MCE) filter paper using a simple lab technique (vacuum filtration) without any surface pretreatment, forming a red AuNP-coated layer. We then investigated the NADH-mediated inhibition on the dissolution of AuNPs coated on the paper, and we found that the size-dependent optical property of AuNPs remains on the paper. 
We further fabricated a device consisting of MCE paper with a wax-encircled, AuNP-coated film atop a cotton absorbent layer, sandwiched between two plastic cover layers. In the absence of $\mathrm{NADH}, \mathrm{Au}^{3+}-\mathrm{CTAB}$ completely dissolved the AuNPs coating, yielding a white readout (Figure 15A). In the presence of $50 \mu \mathrm{M} N A D H$, partial reduction of $\mathrm{Au}^{3+}$ by NADH resulted in only partial dissolution of AuNPs, giving rise to a light pink color (Figure 15B). When challenged with $200 \mu \mathrm{M} \mathrm{NADH}$, all of the $\mathrm{Au}^{3+}$ were reduced by NADH, leaving the AuNPs intact and producing a red readout (Figure 15C). This device exploited capillary force-assisted vertical diffusion, allowing us to apply a $25 \mu \mathrm{L}$ sample to a surface-confined test zone to achieve a detection limit of $12.5 \mu \mathrm{M}$ NADH in under 4 minutes. The enzyme glucose dehydrogenase $(\mathrm{GDH})$ were used as a model to demonstrate that our paper-based device can monitor $\mathrm{NAD}^{+}-$ driven biochemical processes with and without selective dehydrogenase inhibitors by naked-eye observation within 4 minutes at room temperature in a sample volume of $25 \mu \mathrm{L}$. We further demonstrated that the sensor platform could be used in matrix samples such as E. coli cell lysate. We believe that our paper-based device could offer a valuable and low-cost analytical tool for monitoring $\mathrm{NAD}^{+}$-associated enzymatic reactions and screening for dehydrogenase inhibitors in a variety of testing contexts.

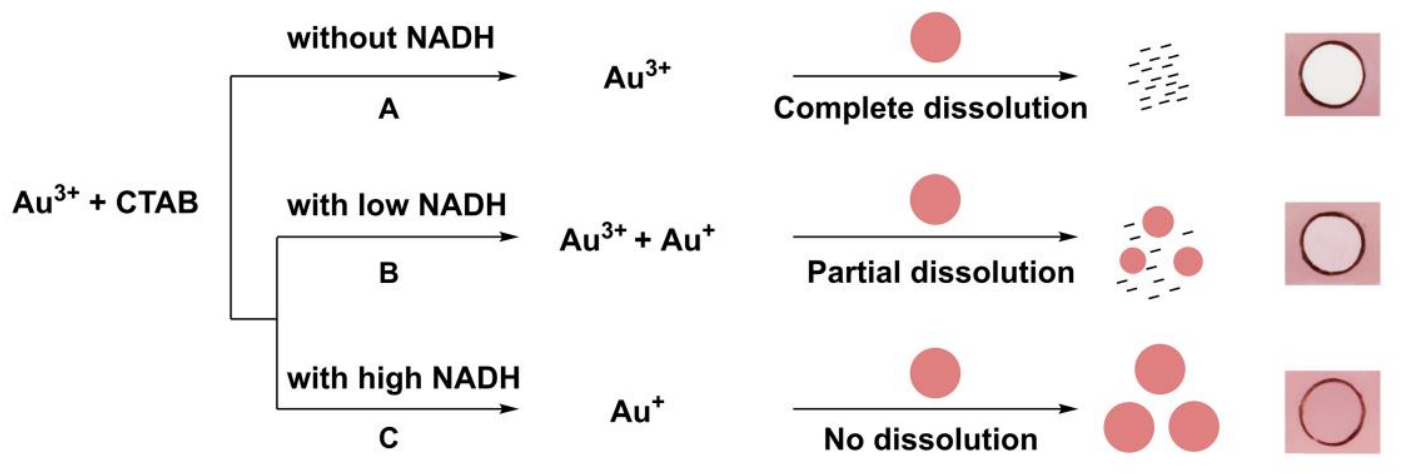

Figure 15. Colorimetric visualization of NADH based on AuNP dissolution on a paper-based device. 


\subsection{Materials and methods}

Chemicals and materials Gold(III) chloride trihydrate $\left(\mathrm{HAuCl}_{4}\right)$, trisodium citrate dihydrate, sodium borohydride $\left(\mathrm{NaBH}_{4}\right)$, cetyltrimethylammonium bromide (CTAB), dihydronicotinamide adenine dinucleotide $(\mathrm{NADH})$, nicotinamide adenine dinucleotide $\left(\mathrm{NAD}^{+}\right)$, glucose, glucose dehydrogenase (from Pseudomonas sp.), mercury(II) acetate, lead(II) acetate trihydrate, silver nitrate, tryptone, sodium chloride, yeast extract and phosphate buffer solution (1.0 M, pH 7.4) were purchased from Sigma-Aldrich and used as received. All solutions were prepared using Milli-Q water (18.2 M $\Omega$ ). Wild-type E. coli strain K12 was used for detection in cell lysate. Mixed cellulose ester (MCE) membrane substrate with $47 \mathrm{~mm}$ diameter, $100 \mathrm{~nm}$ pore size, and $100-\mu \mathrm{m}$ thickness and Amicon Ultra-4 Centrifugal Filter (100KD) were purchased from Millipore. Phano China Marker was purchased from Fisher Scientific.

Synthesis of AuNPs Citrate-capped AuNPs (4.4 $\pm 1.6 \mathrm{~nm})$ were synthesized according to the literature. ${ }^{10}$ All the glasswares were cleaned by incubating with aqua regia solution $\left(\mathrm{HCl}: \mathrm{HNO}_{3}=\right.$ $3: 1$, v/v) overnight followed by rinsing with deionized water. Trisodium citrate solution and $\mathrm{HAuCl}_{4}$ solution were prepared in deionized water and filtered through the syringe filter $(0.22$ $\mu \mathrm{m})$ before use. Specifically, $18 \mathrm{~mL}$ deionized water was added to a $50 \mathrm{~mL}$ Erlenmeyer flask, followed by the addition of $0.5 \mathrm{~mL}$ of $\mathrm{HAuCl}_{4}(10 \mathrm{mM})$ and $0.5 \mathrm{~mL}$ of trisodium citrate $(10 \mathrm{mM})$. After stirring for 5 minutes at $220 \mathrm{rpm}$ at room temperature, $0.5 \mathrm{~mL}$ of ice-cold, freshly prepared $\mathrm{NaBH}_{4}$ solution $(0.1 \mathrm{M})$ was added to the reaction mixture. The color of the resulting solution turned into an orange-red color immediately, indicating the formation of the AuNPs. ${ }^{10}$ The stirring was stopped, and the solution was kept in the dark for 2 hours without disturbing. The trisodium citrate served only as a capping agent since it cannot reduce the gold salt at room temperature. The AuNP solution can be used within 2-5 hours after preparation. The concentration of AuNPs was determined based on the extinction coefficient constant. Particle size distributions were then evaluated using a Zetasizer (Nano-ZS, Malvern Instruments) operating 
with a He-Ne laser at a wavelength of $633 \mathrm{~nm}$ using back-scattered light. Six measurements were made in each run. The size of the AuNPs was characterized by Transmission Electron Microscopy (TEM) (JEM-2100 LaB6) under $200 \mathrm{kV}$ by dropping the solution on the regular copper grids covered with carbon film.

AuNP dissolution in solution phase To observe the dissolution of AuNPs in the $\mathrm{Au}^{3+}$-CTAB solution, the as-prepared AuNPs were concentrated by centrifuging with an Amicon Ultra Centrifugal Filter at $1500 \mathrm{rcf}$ (Eppendorf 5430R). To observe the time course of the AuNP dissolution in the $\mathrm{Au}^{3+}$-CTAB solution, 328 picomoles of concentrated AuNPs were added to a 44.4 mM CTAB solution ( $\mathrm{pH} 4.0$ ) containing 160 nanomoles of $\mathrm{Au}^{3+}$. The spectra were monitored continuously for 60 minutes at room temperature using the UV-Vis spectrometer. To test the effects of $\mathrm{Au}^{3+}$ concentration on AuNP dissolution, identical aliquots of AuNP (328 picomoles) were added into CTAB solution containing 0-200 $\mu \mathrm{M}$ gold salt and incubated for 40 minutes at room temperature while recording the UV-Vis spectra. AuNPs in CTAB solution without $\mathrm{Au}^{3+}$ were used as a reference to calculate the relative absorbance decrease. To test the effect of NADH concentration on AuNP dissolution, freshly prepared NADH (0 to $240 \mu \mathrm{M})$ were added into $200 \mu \mathrm{M} \mathrm{Au}{ }^{3+}$-CTAB solution, followed by adding 328 picomoles AuNPs into this mixture. The UV-Vis spectra of samples were recorded after 40 minutes at room temperature.

NADH-mediated AuNP dissolution on AuNP-coated filter paper MCE substrate was prewetted with deionized water on a Kontes 47-mm Ultra-Ware Microfiltration support base (Kimble Chase). A $1.9 \mathrm{~mL}$ (771.4 picomoles) freshly-made, citrate-capped AuNP solution diluted with $3.1 \mathrm{~mL}$ of $256 \mu \mathrm{M}$ sodium citrate was added to the funnel, and we subsequently prepared the film via ambient vacuum filtration. The film was then cut into 20 small pieces $(5 \mathrm{~mm} \mathrm{~W} \times 6 \mathrm{~mm}$ L) after air-drying for 20 minutes, resulting in strips of film each coated with 21 picomoles AuNPs. To test the effect of $\mathrm{Au}^{3+}$ concentration on the dissolution of AuNPs coated on the paper, these AuNP-coated squares were dropped into $500 \mu \mathrm{L}$ CTAB solution containing different 
concentrations of $\mathrm{Au}^{3+}(0-200 \mu \mathrm{M})$ and then photographed after 40 minutes. The kinetic of AuNPs dissolution on the paper was performed by dropping the AuNP-coated films into $500 \mu \mathrm{L}$ of $200 \mu \mathrm{M} \mathrm{Au}{ }^{3+}-\mathrm{CTAB}$ solution with or without $200 \mu \mathrm{M}$ NADH and then photographed every 10 minutes. In order to test the effect of NADH concentration on AuNP dissolution on the paper, the AuNPs coated films were dropped into $\mathrm{Au}^{3+}-\mathrm{CTAB}$ solutions $(500 \mu \mathrm{L}, 200 \mu \mathrm{M})$ containing different concentrations of NADH and then photographed after 40 minutes.

NADH detection with the paper-based device After preparing the film, we used a wax pencil (Phano China Marker, Fisher Scientific) to draw circles (5 mm diameter) on the AuNPcoated film. The film was then cut into nine pieces $(8 \mathrm{~mm} \mathrm{~W} \times 8 \mathrm{~mm} \mathrm{~L})$. We fabricated a device with four layers listed here from top to bottom: a polyvinyl chloride plastic cover layer $(4.4 \mathrm{~cm} \mathrm{~W}$ $\times 5.4 \mathrm{~cm} \mathrm{~L}$ ) with a 6-mm diameter hole on the top, a prewetted wax-circled AuNP coated film, a prewetted layer of hydrophilic cotton absorbent padding with Kimwipe paper, and a bottom plastic cover. The four layers were held together by clamping the edges of the device with paper clips. The $25 \mu \mathrm{L}$ of $200 \mu \mathrm{M} \mathrm{Au}{ }^{3+}-\mathrm{CTAB}$ solution containing different concentrations of NADH was added into the wax circled reaction zone, and the color change was visualized after 4 minutes. Intensity analysis was performed with ImageJ software (http://imagej.nih.gov/ij/).

GDH-directed NADH production and the inhibition effect of heavy metal ions on the paper-based device The glucose dehydrogenase (GDH) mediated enzymatic reaction was first optimized to determine accurately the enzyme produced NADH concentration. Glucose stock solution $(1 \mathrm{M})$ was prepared in deionized water and kept overnight to allow the mutarotation. The glucose dehydrogenase was dissolved in $10 \%$ BSA. The NAD ${ }^{+}$stock solution and enzyme were prepared right before use. The reaction was performed in $100 \mathrm{mM}$ phosphate buffer $(\mathrm{pH} 7.4)$ at room temperature using the Tecan M1000 PRO microplate reader. The glucose dehydrogenase concentration was investigated from $0.1 \mathrm{U}$ to $0.25 \mathrm{U}$ by monitoring the absorbance at $340 \mathrm{~nm}$ in the presence of $3.9 \mathrm{mM}$ and $7 \mathrm{mM}$ glucose at room temperature. The $\mathrm{NAD}^{+}$concentration was 
further optimized from $3 \mathrm{mM}$ to $5.25 \mathrm{mM}$ by monitoring the absorbance change at $340 \mathrm{~nm}$ in the presence of $3.9 \mathrm{mM}$ and $7 \mathrm{mM}$ glucose. Various concentrations of phosphate buffer $(50 \mathrm{mM}, 100$ $\mathrm{mM}$, and $150 \mathrm{mM}$ at $\mathrm{pH} 7.4)$ and $100 \mathrm{mM}$ phosphate buffer at different $\mathrm{pH}(7,7.4$ and 8$)$ were explored to optimize the enzymatic reaction.

We combined 0.1 U GDH with different concentrations of glucose $(0-20 \mathrm{mM})$ in $100 \mathrm{mM}$ phosphate buffer solution ( $\mathrm{pH}$ 7.4). After incubating at room temperature for 30 minutes, $50 \mu \mathrm{L}$ of the enzymatic reaction solution was mixed with $450 \mu \mathrm{L}$ of different concentration of $\mathrm{Au}^{3+}$ CTAB solution. The AuNP coated films were dipped into the mixture and photographed after 20 minutes. To test the GDH-mediated NADH product on the device, $25 \mu \mathrm{L}$ of the mixture solution were applied to the wax-circled reaction zone on the paper-based device, and the color change was visualized after 4 minutes. Intensity analysis was performed with ImageJ software.

To test the inhibitory effects of heavy metal ions, we incubated different concentrations of $\mathrm{Hg}$ (II) $(0-200 \mu \mathrm{M})$ with $0.1 \mathrm{U}$ GDH for 10 minutes in the presence of $10 \mathrm{mM}$ glucose and 100 $\mathrm{mM}$ phosphate buffer solution ( $\mathrm{pH}$ 7.4) at room temperature. Next, $6 \mathrm{mM} \mathrm{NAD}{ }^{+}$was added to initiate the enzymatic reaction for 15 minutes. The kinetics of the reaction was monitored for the first 6 minutes in microplate reader to calculate the enzyme's activity. The inhibitory effect of different heavy metal ions on GDH was also explored in the same manner. The absorbance change at $340 \mathrm{~nm}$ in the absence of any heavy metal ions was considered as $100 \%$ of the enzyme activity. To demonstrate the inhibitory effects of different concentrations of $\mathrm{Hg}$ (II) on GDHdriven NADH-production on the paper-based device, $10 \mu \mathrm{L}$ of the enzymatic reaction solution were added to $90 \mu \mathrm{L} 400 \mu \mathrm{M} \mathrm{Au}{ }^{3+}-\mathrm{CTAB}$ solution, and $25 \mu \mathrm{L}$ of this solution was applied to the test zone. The color intensity analysis was performed with ImageJ after 4 minutes of reaction. We also tested $\mathrm{Ag}(\mathrm{I})$ and $\mathrm{Pb}(\mathrm{II})$ as potential inhibitors in a similar fashion and evaluated the inhibitory effects of these metals by measuring the decrease in the color intensity produced by the enzyme-substrate reaction. 


\section{Detection of NADH and GDH-directed NADH production in $20 \%$ cell lysate Wild-type}

E. coli strain K-12 was cultured in $50 \mathrm{~mL}$ of autoclaved L-broth (Luria) media (10 g/L tryptone, 5 $\mathrm{g} / \mathrm{L}$ yeast extract, and $10 \mathrm{~g} / \mathrm{L} \mathrm{NaCl}$ ) in an Erlenmeyer flask and was harvested after 24 hours of incubation at $37{ }^{\circ} \mathrm{C}$ with orbital shaking. We obtained an optical density $\left(\mathrm{OD}_{600}\right)$ reading of 1.62 with an approximate cell density of $1.62 \times 10^{9}$ cells $/ \mathrm{mL}$. The resultant cell suspension was centrifuged at $8500 \times \mathrm{g}$ for 15 minutes at $4{ }^{\circ} \mathrm{C}$. The cell pellet was collected and resuspended in $1.62 \mathrm{~mL}$ of $100 \mathrm{mM}$ phosphate buffer solution $(\mathrm{pH}$ 7.4) to perform the cell-lysis with an ultrasonic cell disruptor (Misonix Microson XL2000) for 10 seconds pulse with 30 seconds intervals for 4 times in an ice-water bath. The cell lysate was centrifuged at $5000 \times \mathrm{g}$ for 10 minutes at $4^{\circ} \mathrm{C}$ to remove the supernatant. The final decanted supernatant was diluted with phosphate buffer $(100 \mathrm{mM}, \mathrm{pH} 7.4)$ to make $20 \%(\mathrm{v} / \mathrm{v})$ cell lysate $\left(\sim 1 \times 10^{10}\right.$ cells $\left./ \mathrm{mL}\right)$. The detection of NADH and GDH-directed NADH production were performed as described above except the reaction buffer was replaced with $20 \%$ cell lysate.

\subsection{Results and discussions}

\subsubsection{NADH-mediated AuNP dissolution in solution}

It has been reported that acidic $\mathrm{Au}^{3+}-\mathrm{CTAB}$ solution can dissolve AuNPs, ${ }^{53}$ and we recently discovered that $\mathrm{NADH}$ can reduce the $\mathrm{Au}^{3+}$ in the solution, which greatly inhibited AuNP dissolution at room temperature. ${ }^{84}$ Thus, a colorimetric sensor was developed on the basis of NADH- mediated AuNP dissolution in $\mathrm{Au}^{3+}-\mathrm{CTAB}$ solution.

\subsubsection{Characterization of citrate-capped AuNPs}

AuNPs $(4.4 \pm 1.6 \mathrm{~nm}$ in diameter) were used as the signaling reporter since it is known that smaller sizes dissolve faster. ${ }^{53,}{ }^{246}$ The UV-Vis spectrometer is used to characterize the concentration of the AuNPs. The maximum absorption peak of the prepared AuNP was located at $504 \mathrm{~nm}$ (Figure 16A). The concentration of the AuNPs was calculated to be $41 \mu \mathrm{M}$ according to Beer's Law. Dynamic light scattering (DLS), which is based on the Brownian motion of particles, 
is used to determine the nanoparticles' size as well as how the scattering fluctuates with time on the basis of constantly changing distances of the scatters. The hydrodynamic diameter of the AuNPs obtained from the Zetasizer measurement is $7.5 \mathrm{~nm}$ (Figure 16B). The obtained polydispersity index (PDI) is 0.063 , indicating a narrow size distribution.
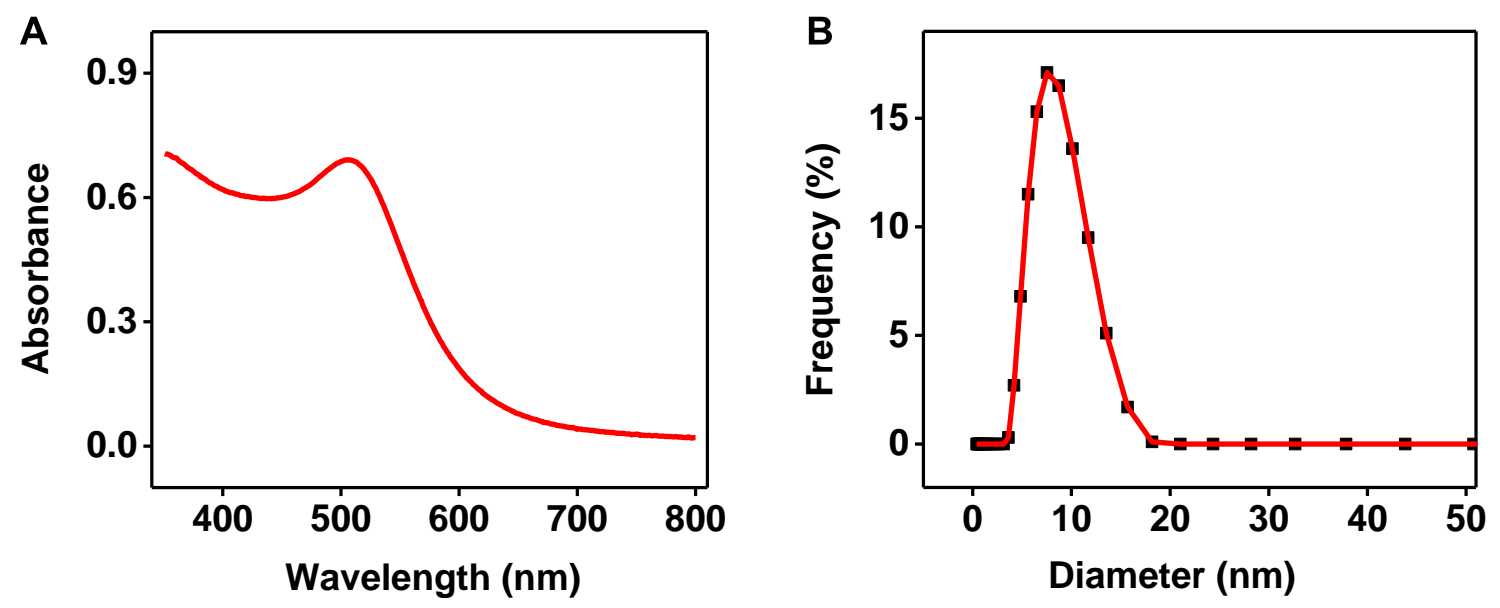

Figure 16. Characterization of the concentration and size of these synthesized AuNPs. (A) UVVis spectrum of the AuNPs with a maximum absorbance at $504 \mathrm{~nm}$. (B) Size distribution of the AuNPs measured using Zetasizer: the average size is $7.5 \mathrm{~nm}$ and its polydispersity index (PDI) is 0.063 .

Transmission Electron Microscopy (TEM) is a good tool for characterization nanomaterials to obtain a quantitative measurement including particle morphology and size distribution. The AuNP with desired spherical shape were observed to be monodispersed (Figure 17A). The size was determined by Nano Measurer 1.2. The analysis of the image confirmed that they are uniformly sized (diameter: $\sim 4 \pm 1.6 \mathrm{~nm}$, Figure 17B). However, the value is smaller compared to the Zetasizer measurement. ${ }^{247}$ 
A

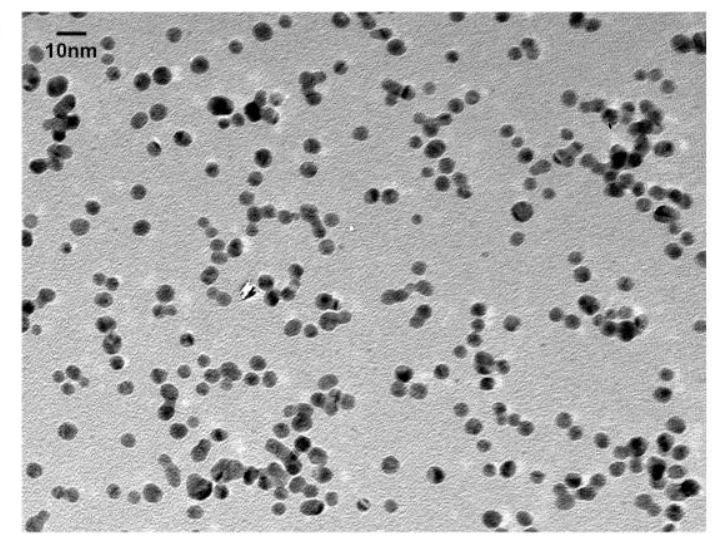

B

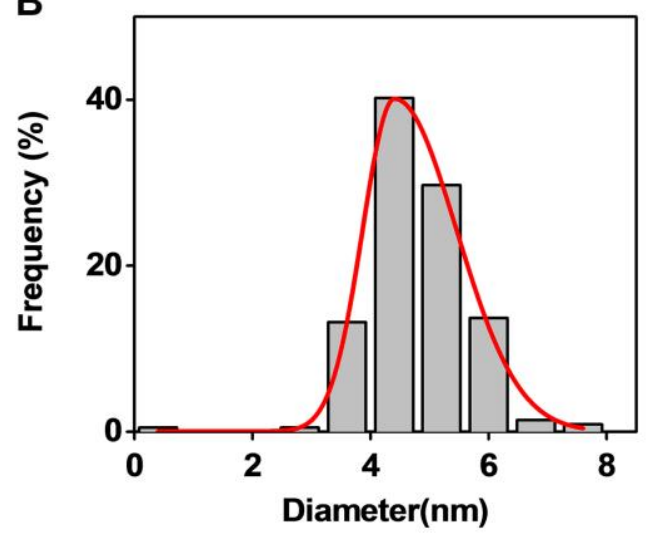

Figure 17. Size characterization of the freshly prepared AuNPs. (A) TEM image of the AuNPs. (B) Size distribution of the AuNPs.

\subsubsection{Time course of AuNP dissolution in $\mathrm{Au}^{3+}-\mathrm{CTAB}$ solution}

It is noteworthy that AuNP dissolution requires a higher $\mathrm{CTAB}$ concentration than its critical micelle concentration ${ }^{248}$ to form micelles that carry $\mathrm{Au}^{3+}$ ions. When we added 160 nanomoles of $\mathrm{Au}^{3+}$ into a $44.4 \mathrm{mM} \mathrm{CTAB}$ solution ( $\left.\mathrm{pH} 4.0\right)$, the solution turned a yellow color and the resulting $\mathrm{Au}^{3+}-\mathrm{CTAB}$ complex — more specifically, the $\mathrm{AuBr}_{4}{ }^{-}$anion—exhibited strong absorbance at 394 $\mathrm{nm}$ with a distinct shoulder at $450 \mathrm{~nm} .{ }^{249}$ Upon adding 328 picomoles of AuNPs, the plasmon peak of the AuNPs shifted from $504 \mathrm{~nm}$ to $526 \mathrm{~nm}$ in the $\mathrm{Au}^{3+}$-CTAB solution. The absorbance of both the $\mathrm{Au}^{3+}-\mathrm{CTAB}$ complex at $394 \mathrm{~nm}$ and the AuNPs at $526 \mathrm{~nm}$ gradually decreased over the course of the reaction (Figure 18A). Under reaction conditions where the $\mathrm{Au}^{3+}$ :AuNP ratio was 489:1, we found that the absorbance at $526 \mathrm{~nm}$ decreased rapidly, and approximately $73.5 \%$ of the AuNPs were dissolved in the first 10 minutes. The dissolution then slowed down and started to reach its plateau after 40 minutes. No detectable change was observed after 60 minutes, clearly indicating that all AuNPs were dissolved (Figure 18B). In contrast, we observed no dissolution of AuNPs in CTAB solution without $\mathrm{Au}^{3+}$ and used this as a reference to calculate the relative absorbance decrease at $526 \mathrm{~nm}$. 

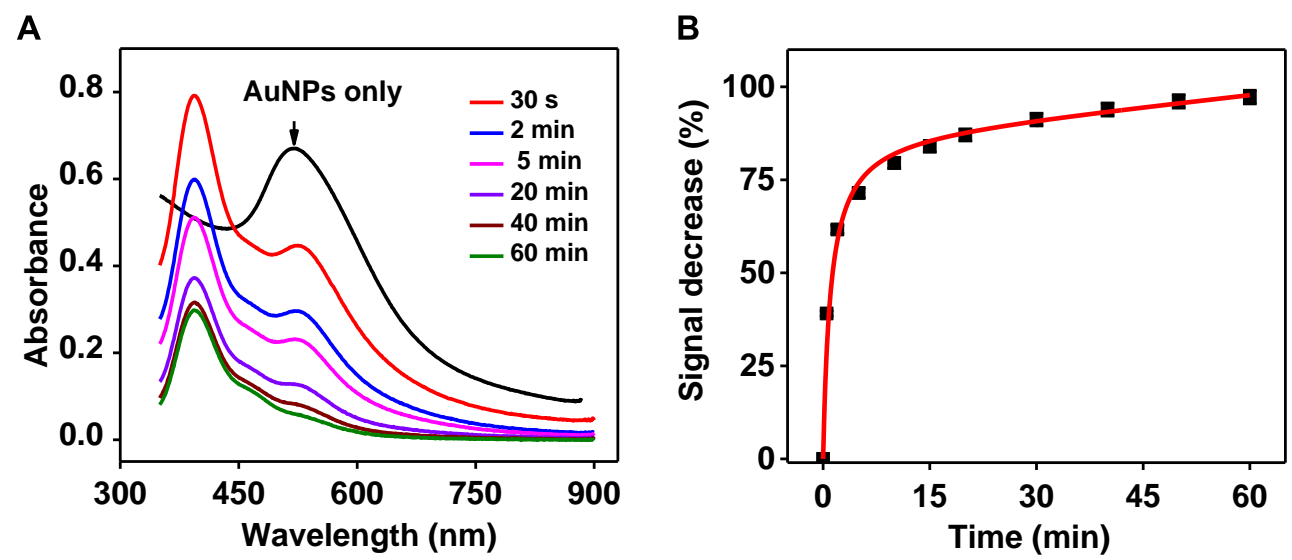

Figure 18. Time course of AuNP dissolution in $\mathrm{Au}^{3+}-\mathrm{CTAB}$ solution. (A) UV-Vis spectra recorded at different time points after adding AuNPs into $\mathrm{Au}^{3+}$-CTAB solution. The black line represents the spectrum for AuNPs in CTAB alone. (B) Time course of the signal decrease at 526 nm. AuNPs in CTAB solution alone was used as a reference to calculate the relative signal decrease.

\subsubsection{Effect of $\mathrm{Au}^{3+}$ concentration on AuNP dissolution}

The concentration of $\mathrm{Au}^{3+}$ played an important role in AuNP dissolution. In order to test the $\mathrm{Au}^{3+}$ concentration effect on AuNPs dissolution, we therefore optimized the $\mathrm{Au}^{3+}$ :AuNP ratio in a homogeneous solution to achieve a much shorter reaction time. The results demonstrated that AuNP dissolution increased as the $\mathrm{Au}^{3+}$ concentration increased. Compared with AuNPs alone, the absorbance at $526 \mathrm{~nm}$ was greatly decreased but still detectable when the $\mathrm{Au}^{3+}$ concentration was in the range of 40 to $160 \mu \mathrm{M}$ (Figure 19A). As the $\mathrm{Au}^{3+}$ concentration increased to $200 \mu \mathrm{M}$ (an $\mathrm{Au}^{3+}:$ AuNP ratio of $610: 1, \mathrm{~mol} / \mathrm{mol}$ ), the SPR peak completely disappeared, indicating that all of the AuNPs were dissolved (Figure 19A). AuNPs in CTAB solution without $\mathrm{Au}^{3+}$ was used as a reference to calculate the relative absorbance decrease at $526 \mathrm{~nm}$. We observed that as the $\mathrm{Au}^{3+}$ concentration increases, the AuNP dissolution also increases. It is clear that a high concentration of $\mathrm{Au}^{3+}$ promoted a quick dissolution of AuNPs at room temperature (Figure 19B), Thus $\mathrm{Au}^{3+}:$ AuNPs $(610: 1 \mathrm{~mol} / \mathrm{mol})$ was employed in the subsequent experiments. 

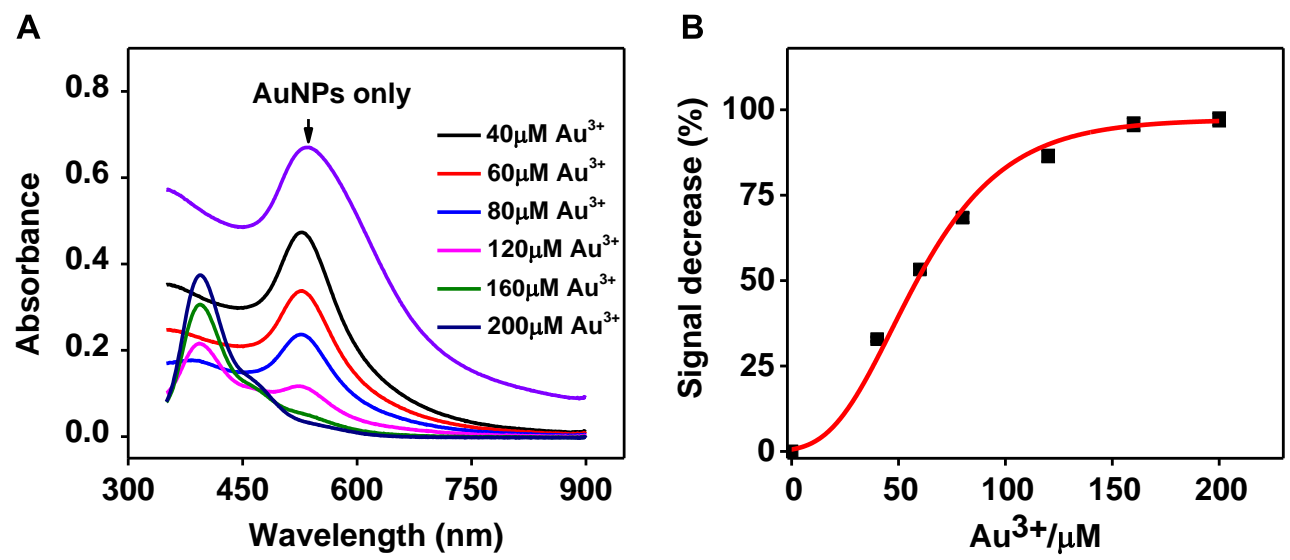

Figure 19. The effect of $\mathrm{Au}^{3+}$ concentration on AuNP dissolution in CTAB. (A) UV-Vis spectra of 328 picomoles of AuNPs in the presence of $\mathrm{Au}^{3+}$ concentrations ranging from $0-200 \mu \mathrm{M}$. (B) Absorbance at $526 \mathrm{~nm}$ steadily decreased as the concentration of $\mathrm{Au}^{3+}$ increased.

\subsubsection{Effect of NADH concentration on AuNP dissolution}

It is known that $\mathrm{Au}^{3+}$ can dissolve AuNPs in an acidic CTAB solution whereas its reduced form $\left(\mathrm{Au}^{+}\right)$is unable to perform such dissolution. ${ }^{53}$ Xiao et al. previously reported that NADH facilitated the rapid reduction of $\mathrm{Au}^{3+}$ to $\mathrm{Au}^{+}$in $\mathrm{CTAB}$ solution. ${ }^{22}$ We, therefore, predicted that the presence of NADH would inhibit the dissolution of AuNPs in an $\mathrm{Au}^{3+}-\mathrm{CTAB}$ solution.
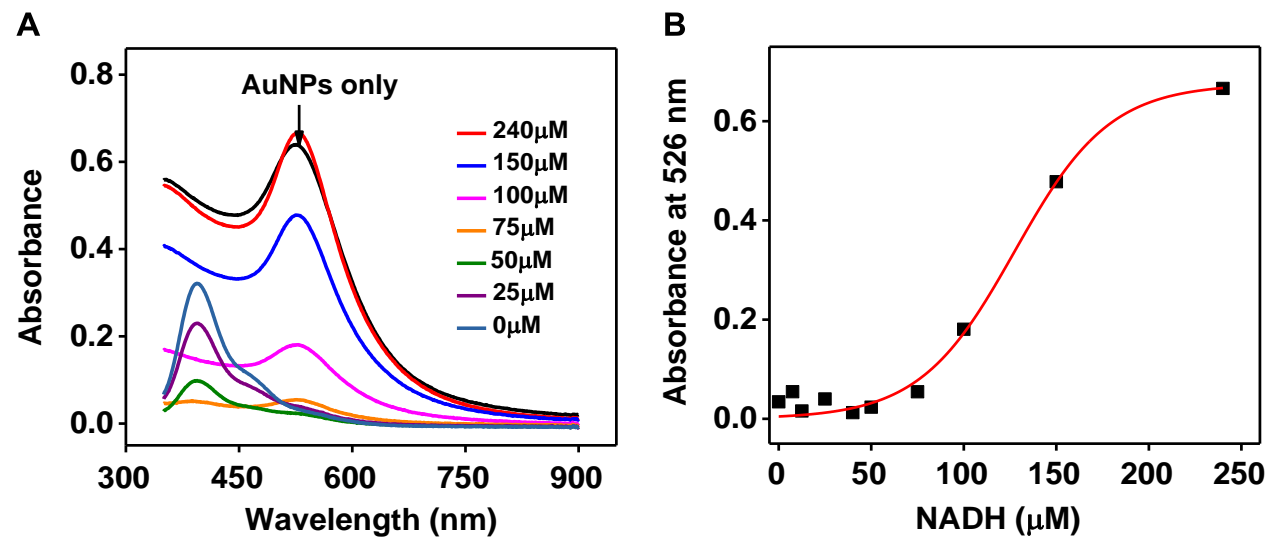

Figure 20. The effect of NADH concentration on AuNP dissolution. (A) UV-Vis spectra recorded from $200 \mu \mathrm{M} \mathrm{Au}{ }^{3+}$ in CTAB with different concentrations of NADH after 40 minutes of reaction with 328 picomoles of AuNPs at room temperature. (B) Absorbance measurements at $526 \mathrm{~nm}$ for AuNPs in the presence of different concentrations of NADH. 
Thus, the dissolution of AuNPs in the solutions containing different concentrations of NADH has been explored. We mixed different concentration of NADH solution with a $200 \mu \mathrm{M}$ gold salt solution, followed by the addition of 328 picomoles AuNPs, and recorded the UV-Vis spectra of the samples after 40 minutes at room temperature (Figure 20A). When NADH concentrations were below $75 \mu \mathrm{M}$, only a small quantity of $\mathrm{Au}^{3+}$ was reduced to $\mathrm{Au}^{+}$. The excess $\mathrm{Au}^{3+}$ left in the solution was sufficient to dissolve most of the AuNPs. The absorbance of the small amount of AuNPs left in the solution under these conditions became difficult to measure accurately due to the strong interference from the distinct shoulder of the $\mathrm{Au}^{3+}-\mathrm{CTAB}$ complex at $450 \mathrm{~nm}$. When the NADH concentration was in the range of $100 \mu \mathrm{M}$ to $150 \mu \mathrm{M}$, the amount of unreacted $\mathrm{Au}^{3+}$ decreased in the solution, and partial dissolution of AuNPs occurred. AuNP dissolution was further inhibited with the increasing of NADH concentrations; interestingly, we observed a higher AuNP absorbance after 40 minutes upon addition of $240 \mu \mathrm{M}$ NADH relative to the absorbance of AuNPs alone in CTAB solution. This is because the excess NADH first completely reduced the $\mathrm{Au}^{3+}$ to $\mathrm{Au}^{+}$, and then further reduced the $\mathrm{Au}^{+}$to $\mathrm{Au}^{0}$. This resulted in the enlargement of the AuNPs, consistent with previously reported findings. ${ }^{22}$ By monitoring the absorbance change at $526 \mathrm{~nm}$, we could identify the NADH concentrations above $75 \mu \mathrm{M}$ using NADH-mediated AuNP dissolution (Figure 20B). However, a large amount of sample (1000 $\mu \mathrm{L})$ was required to be measured via UV-Vis spectrometer.

\subsubsection{NADH-mediated AuNP dissolution on paper}

To achieve the detection in an instrument-free manner, we transferred the NADH-inhibited dissolution process onto MCE filter paper via vacuum filtration and tested the NADH inhibitory effect on the dissolution of AuNPs on MCE filter paper in an $\mathrm{Au}^{3+}-\mathrm{CTAB}$ solution. We also used the enzyme glucose dehydrogenase as a model to demonstrate that our sensor platform can monitor the production of $\mathrm{NADH}$ via $\mathrm{NAD}^{+}$-driven biochemical processes. 


\subsubsection{AuNP deposition on the MCE filter paper}

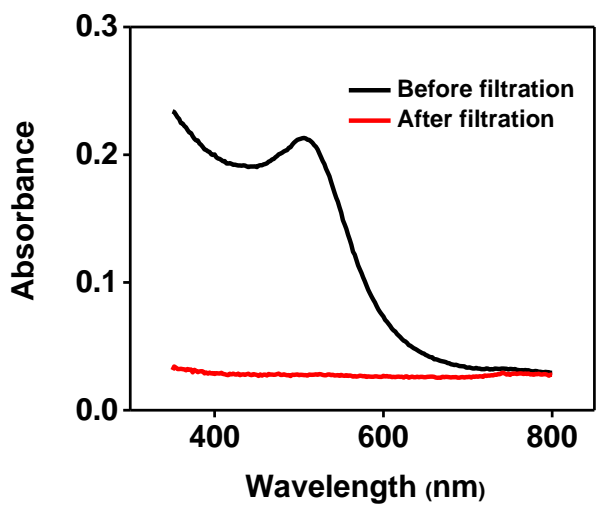

Figure 21. UV-Vis spectrum of the AuNP solution before and after vacuum filtration.

Previous work has shown that films made by vacuum filtration generally offer good homogeneity, strong adhesive strength, massive scalability, excellent stability and reproducibility. ${ }^{250,}{ }^{251}$ When adding the AuNP solution to the MCE substrate through vacuum filtration, the uniform pore size of MCE paper allows stable and reproducible liquid flow through the membrane, and a smooth and even AuNP layer can be rapidly formed on the MCE surface. UV-Vis experimental results demonstrated that there was no leaking of AuNPs through filter paper during the film preparation (Figure 21). The film was then cut into 20 small pieces $(5 \mathrm{~mm}$ $\mathrm{W} \times 6 \mathrm{~mm} \mathrm{~L}$ ) after air-drying for 20 minutes, resulting in strips of film, each coated with 21 picomoles AuNPs.

\subsubsection{Effect of $\mathrm{Au}^{3+}$ concentration on AuNPs dissolution on the paper}

The $\mathrm{Au}^{3+}$ concentration in the CTAB solutions also played an important role in AuNP dissolution on the paper. We observed that as the $\mathrm{Au}^{3+}$ concentration increased, the dissolution of AuNPs coated on the paper were also increased, resulting in a color readout that shifted from red to light pink. When the $\mathrm{Au}^{3+}$ concentration reached to $200 \mu \mathrm{M}$, all of the AuNPs were dissolved from the surface, leaving the paper a white color (Figure 22). Thus, $200 \mu \mathrm{M} \mathrm{Au}^{3+}-\mathrm{CTAB}$ solution were used for subsequent experiments. 


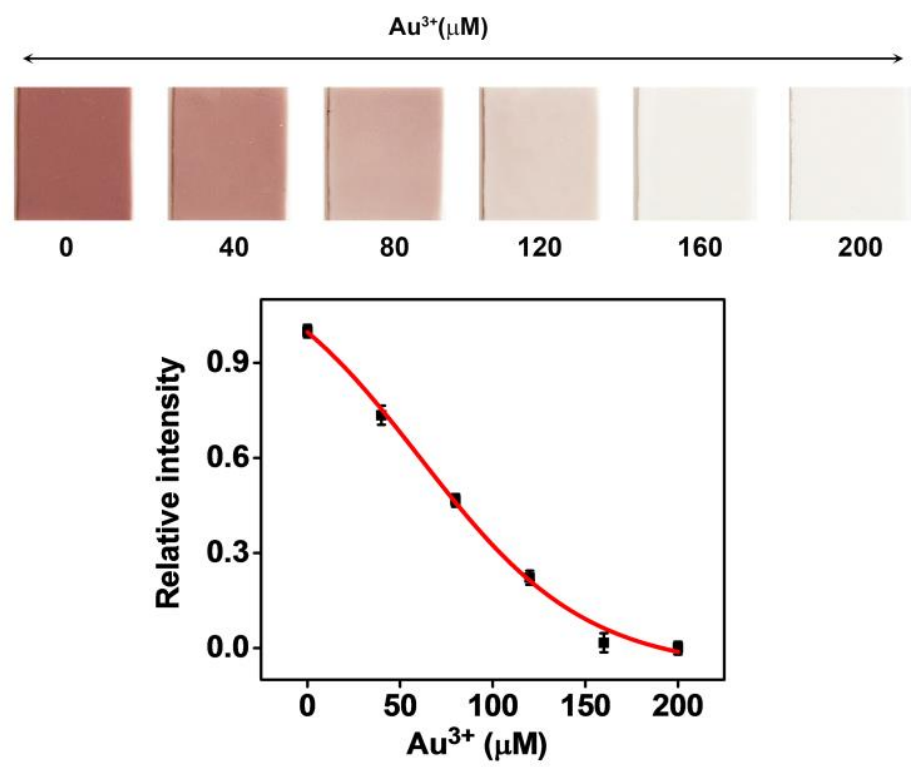

Figure 22. Dissolution of AuNP-coated MCE films at different $\mathrm{Au}^{3+}$ concentrations. Incubation with $500 \mu \mathrm{L}$ CTAB solution containing increasing $\mathrm{Au}^{3+}$ concentrations resulted in an increased dissolution and associated color loss after 40 minutes.

\subsubsection{Time course of AuNP dissolution on the paper}

The dissolution of AuNPs on the paper was increased as the reaction time was increased. The kinetic of AuNP dissolution on the paper was performed by dipping the AuNP-coated films into $500 \mu \mathrm{L}$ of $200 \mu \mathrm{M} \mathrm{Au}^{3+}$-CTAB solution with or without $200 \mu \mathrm{M}$ NADH and then photographed every 10 minutes. The intensity of the film was analyzed using the ImageJ software. We found that the AuNPs coated on the MCE film were completely dissolved after 40 minutes and left a white background in the absence of NADH (Figure 23). In contrast, the dissolution was completely inhibited in the presence of $200 \mu \mathrm{M}$ NADH and left the AuNPs intact on the paper (Figure 23). The relative intensity was calculated by normalizing the unreacted AuNP-coated film to 1 and the AuNP-coated film in the absence of NADH to 0. 


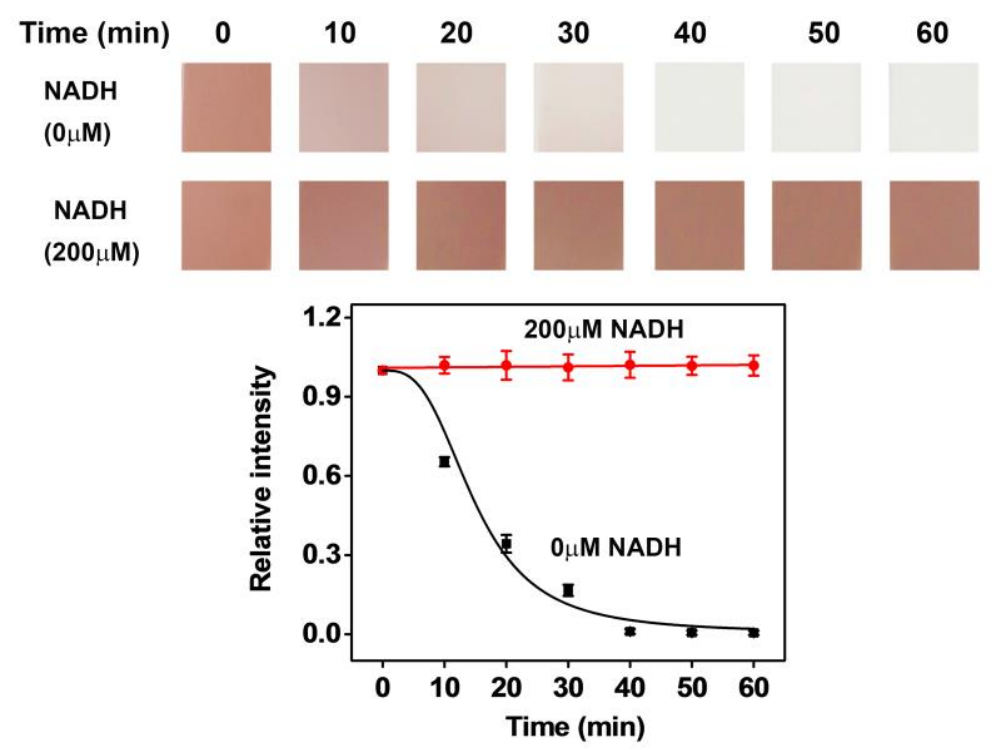

Figure 23. Time course of the reaction on AuNP-coated MCE filter paper in the absence and presence of $200 \mu \mathrm{M}$ NADH in $200 \mu \mathrm{M} \mathrm{Au}^{3+}$-CTAB solution.

\subsubsection{Effect of NADH concentration on AuNP dissolution on the paper}

In order to test the NADH inhibitory effect on AuNP dissolution on the paper, the AuNP-

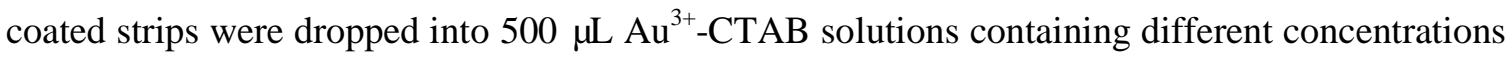
of NADH. In the absence of NADH, $\mathrm{Au}^{3+}-\mathrm{CTAB}$ solution dissolved all of the AuNPs coated on the paper, leaving a white color (Figure 24). In the presence of NADH, it rapidly reduced $\mathrm{Au}^{3+}$ to $\mathrm{Au}^{+}$, inhibiting the dissolution of AuNPs coated on the paper and resulting in a color readout that shifted from light pink to red (Figure 24). Although the reaction took up to 40 minutes, the color difference was readily detectable by the naked-eye with a detection limit of $7.5 \mu \mathrm{M}$, which is 10 fold lower than the reported value of a solution-based colorimetric NADH sensor. ${ }^{22}$ No clear growth of the AuNPs on the paper was observed even with the highest concentration of NADH $(200 \mu \mathrm{M})$ since the growth of AuNPs usually required several hours to overnight incubation. We believe that a 40-minute reaction time was too short for AuNPs to grow on the paper. Although this colorimetric assay was mainly designed to provide rapid semi-quantitative results (i.e., dark 
red color indicated that more target molecule was present), it is possible to obtain quantitative analysis by using color image managing software such as ImageJ.

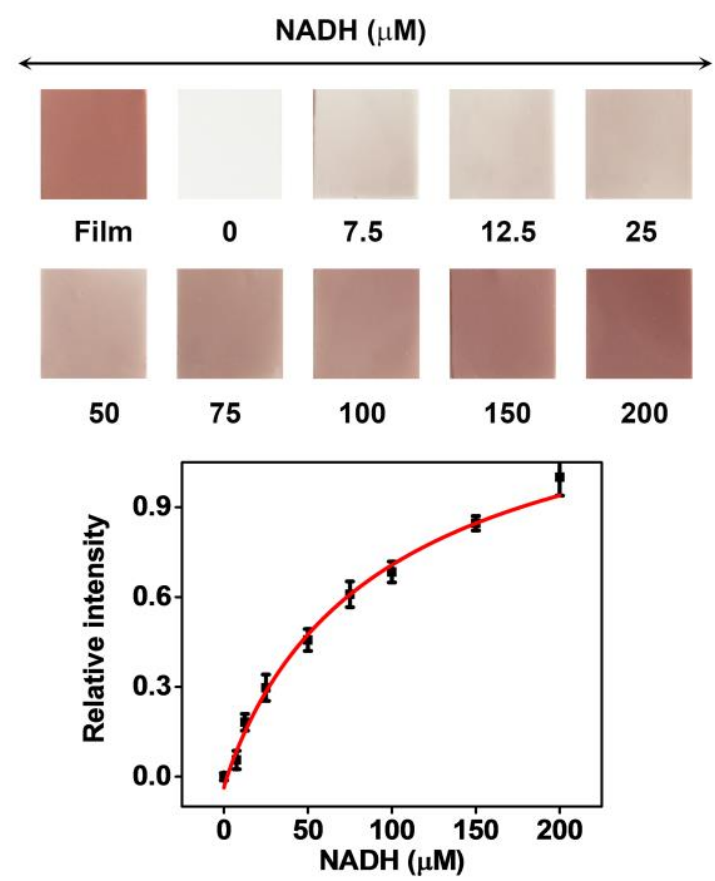

Figure 24. Colorimetric detection of NADH on AuNP-coated MCE filter paper. Increasing the NADH concentration from 0 to $200 \mu \mathrm{M}$ in a $200 \mu \mathrm{M} \mathrm{Au}{ }^{3+}-\mathrm{CTAB}$ solution increasingly inhibited the dissolution of AuNPs coated on the paper, resulting in a stronger red color readout.

\subsubsection{Effect of GDH-mediated NADH production on paper-based AuNP dissolution}

Glucose dehydrogenase (GDH, EC 1.1.1.47), an enzyme that can catalyze the oxidation of $\beta$ D-glucose to gluconic acid while reducing $\mathrm{NAD}^{+}$to $\mathrm{NADH}$ which has a strong absorbance at $340 \mathrm{~nm}$. The glucose concentration determines the amount of NADH produced. ${ }^{126}$ To achieve the best enzymatic condition, the enzyme concentration was first optimized. We chose $3.9 \mathrm{mM}$ and 7 $\mathrm{mM}$ of glucose to perform the enzyme reaction and monitored the absorbance change at $340 \mathrm{~nm}$. We observed that a higher reaction rate was obtained with a higher concentration of GDH (Figure $25 \mathrm{~A})$. Although a low concentration of GDH $(0.1 \mathrm{U})$ reacted slowly, it produced the largest absorbance difference at $340 \mathrm{~nm}$ between $3.9 \mathrm{mM}$ and $7 \mathrm{mM}$ with a much longer detection window. Thus, $0.1 \mathrm{U}$ of the enzyme was used for subsequent experiments. 

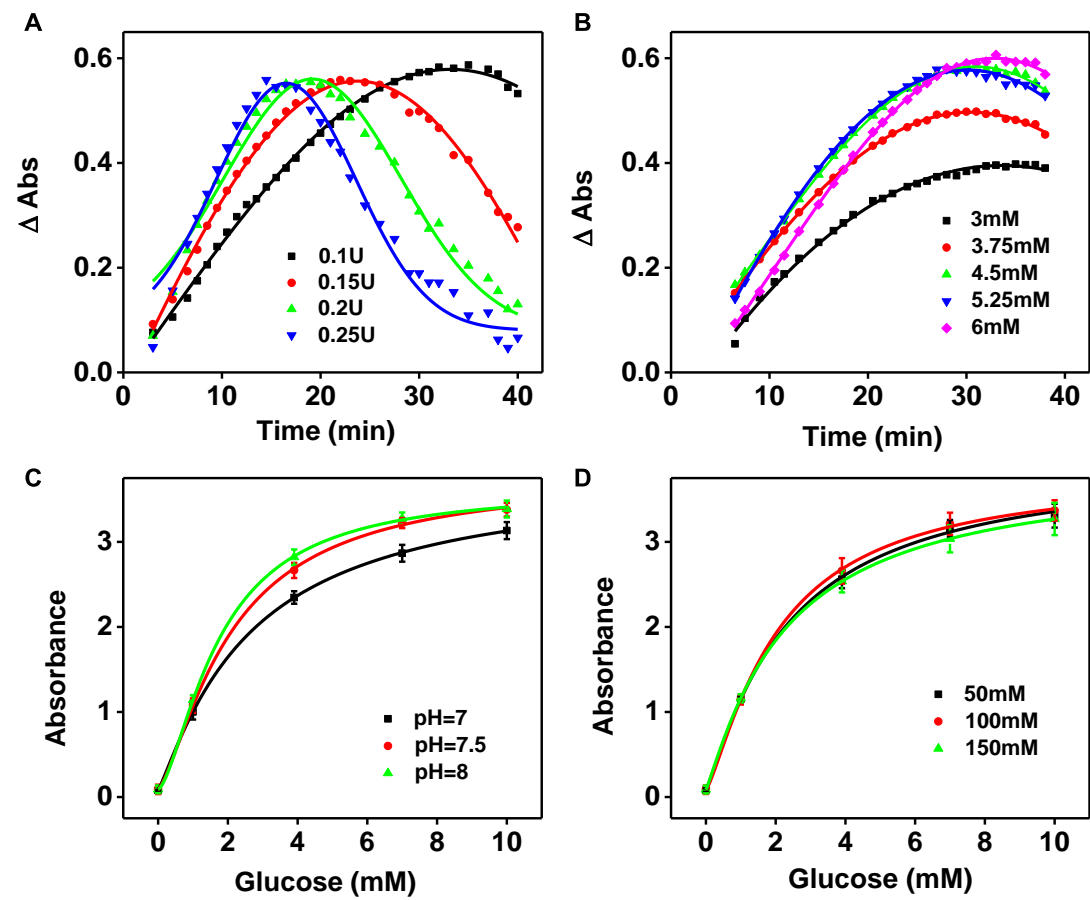

Figure 25. Optimization of GDH catalyzed reactions by monitoring the absorbance of NADH at $340 \mathrm{~nm}$. (A) Optimization of GDH concentration by comparing the absorbance difference of NADH between $3.9 \mathrm{mM}$ glucose and $7 \mathrm{mM}$ glucose in the presence of $4.5 \mathrm{mM} \mathrm{NAD}^{+}$; $(\mathbf{B})$ Optimization of $\mathrm{NAD}^{+}$concentration by comparing the absorbance difference of NADH between $3.9 \mathrm{mM}$ and $7 \mathrm{mM}$ glucose in the presence of $0.1 \mathrm{U} \mathrm{GDH}$; (C) Effect of buffer $\mathrm{pH}$ on the enzymatically produced NADH in the presence of $4.5 \mathrm{mM} \mathrm{NAD}^{+}, 0.1 \mathrm{U}$ GDH and $0-10 \mathrm{mM}$ glucose; (D) Effect of buffer concentration on the enzymatically produced NADH in the presence of 4.5 mM NAD ${ }^{+}, 0.1 \mathrm{U}$ GDH and 0-10 mM glucose.

$\mathrm{NAD}^{+}$is an important cofactor to accept electrons and form NADH in the enzymatic reaction. Thus, the NAD ${ }^{+}$concentration was also optimized by using two concentrations of glucose $(3.9$ $\mathrm{mM}$ and $7 \mathrm{mM}$ ). By monitoring the absorbance change at $340 \mathrm{~nm}$, it was clear that when the $\mathrm{NAD}^{+}$concentration increased, the absorbance difference at $340 \mathrm{~nm}$ also increased. The $\mathrm{NAD}^{+}$ concentration $(4.5 \mathrm{mM})$ was used for subsequent experiments to obtain maximum absorbance difference (Figure 25B).

Since the activity of GDH is dependent on the buffer $\mathrm{pH}$, phosphate buffer $(100 \mathrm{mM})$ with varying $\mathrm{pH}$ were also examined in the presence of $0.1 \mathrm{U}$ of $\mathrm{GDH}$ and $4.5 \mathrm{mM} \mathrm{NAD}{ }^{+}$. 
Analogously, the absorbance at $340 \mathrm{~nm}$ after 30 minutes reaction was plotted versus glucose concentration. The results demonstrated that the reaction rate also increased as the $\mathrm{pH}$ increased from 7 to 7.4. However, when we further increased the $\mathrm{pH}$ to 8 , no significant improvement was observed (Figure 25C). The concentration of phosphate buffer was also optimized in the similar fashion. The absorbance at $340 \mathrm{~nm}$ developed at room temperature after 30 minutes was plotted with different glucose concentration. We did not observe any clear difference when changing the concentration of the phosphate buffer (Figure 25D). Thus, phosphate buffer (100 mM, pH 7.4) was used for subsequent experiments.

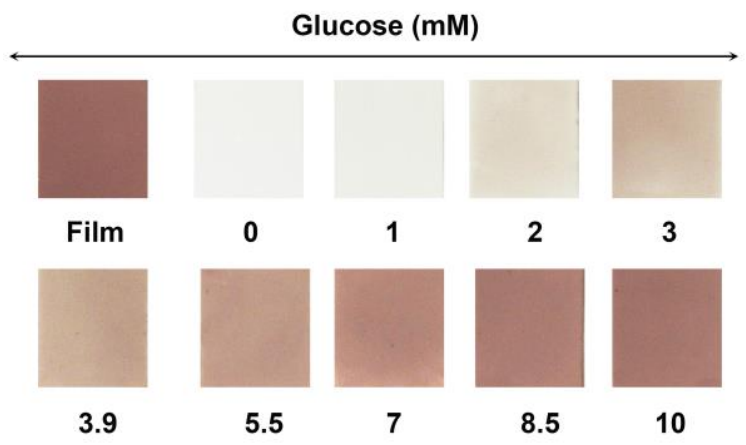

Figure 26. Glucose detection in $400 \mu \mathrm{M} \mathrm{Au}{ }^{3+}-\mathrm{CTAB}$ after a 20-minute reaction. Increasing the glucose concentration from 0 to $10 \mathrm{mM}$ in a $400 \mu \mathrm{M} \mathrm{Au}{ }^{3+}$-CTAB solution increasingly inhibited the dissolution of AuNPs coated on the paper, resulting in a stronger red color readout.

Once the experimental condition was optimized, we visually monitored dehydrogenasedriven NADH production using AuNP-coated paper. Different concentrations of glucose were used to produce different amount of NADH in the presence of GDH; the samples were mixed with the $\mathrm{Au}^{3+}-\mathrm{CTAB}$ solution and then incubated with the AuNP-coated films. Since no NADH was generated in the absence of glucose, all of the coated-AuNP were fully dissolved from the paper surface. Along with the increasing glucose concentration, the NADH production was also increased. Thus, the color of the AuNP-coated film changed from a light pink to red and eventually dark red with the increasing of glucose concentration (2 mM - $10 \mathrm{mM})$ (Figure 26). 
It has been successfully demonstrated that NADH and enzyme-generated NADH can inhibit the dissolution of AuNPs coated on the filter paper. With the described paper-based AuNP dissolution method, we obtained a 10 time-lower detection limit compared to the solution-based method. However, the detection required 40 minutes to complete AuNP dissolution, which is not suitable for an on-site screening test.

\subsubsection{Using paper-based device to monitor NADH-related enzymatic reaction}

To shorten the reaction time on the paper-based sensor and efficiently visualize the presence of NADH in the microliter-scale sample, we have successfully developed a paper-based device that can uniformly and rapidly detect microliter-scale samples within a surface-confined test zone within 4 minutes.

\subsubsection{Confinement of microliter sample within the test zone}

The porous paper offers limited control over of the fluid transportation rate and direction, and the lateral diffusion of fluids in the paper is usually much faster than vertical diffusion. ${ }^{252} \mathrm{We}$ found when a $25 \mu \mathrm{L}$ of $\mathrm{Au}^{3+}-\mathrm{CTAB}$ solution was placed on the top of the AuNP-coated paper, a narrow white circle was observed at the edge of the droplet after 10 minutes, and that $46 \%$ of the AuNPs dissolved in the reaction area (Figure 27A). Most of the $\mathrm{Au}^{3+}-\mathrm{CTAB}$ micelles, which are typically $\sim 3 \mathrm{~nm}$ in diameter ${ }^{253}$ presumably migrated to the edge of the droplet due to the "coffee ring effect", ${ }^{254}$ such that particles in this region are more likely to be dissolved than those in the center.

To work with a small volume of sample, we need to be able to confine the liquid within the test zone to avoid the lateral escape of reactants. The best way to accomplish this goal is to create a closed hydrophobic barrier both on the surface of the AuNP-coated film and across the thickness of the paper. This could be achieved via wax printing, a rapid and inexpensive technique for large-scale production of microfluidic paper-based analytical devices. ${ }^{252}$ However, wax printing requires a time-consuming heat penetration step, and it is generally difficult to form 
a good hydrophobic barrier across the thickness of the paper. ${ }^{252}$ As a simple alternative, the hydrophobic barrier could be drawn on the surface of AuNP-coated paper by a wax pencil to confine the reactants, which could be done in 5 seconds with good reproducibility and no restriction on the thickness of the circle. Thus, after preparing the film, a wax pencil was used to draw circles (5 mm diameter) on the AuNP-coated paper. The experimental data showed that a droplet of $25 \mu \mathrm{L} \mathrm{Au}{ }^{3+}$-CTAB solution could be successfully retained within the wax-circled test zone for up to 21 minutes, with $58 \%$ of the AuNPs dissolved (Figure 27B). The wax circle on the top of the filter paper does confine the sample and allows the solution to diffuse vertically, rather than laterally, through the AuNP-coated test zone. However, the lateral escape of the confined reactants from underneath the wax barrier was also observed. It was also noticed that the dissolution still occurred mainly around the edge of the wax-circled test zone while the center of the film remained intact.

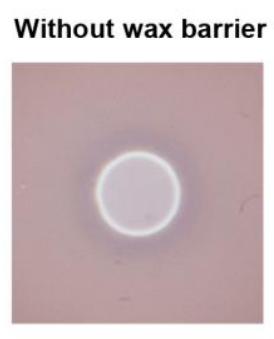

(A)

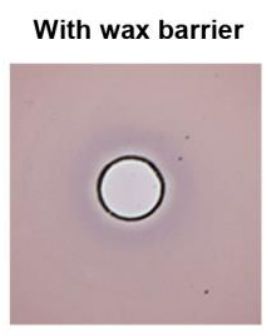

(B)

Figure 27. AuNP dissolution limited by lateral diffusion due to reactant escape either with or without a wax-circled test zone. We added a droplet of $25 \mu \mathrm{L}$ of $200 \mu \mathrm{M} \mathrm{Au}{ }^{3+}$-CTAB solution to a film without (A) or with (B) a wax-circled test zone at room temperature.

\subsubsection{Absorbent effect on lateral diffusion}

To further eliminate lateral diffusion in the confined test zone from either side of the filter paper, a hydrophilic absorbent layer underneath the wax-circled AuNP-coated film was added to enhance the vertical transport of reactants through the paper. Since water has at least 10-fold higher self-diffusion coefficient ${ }^{255}$ than CTAB micelles, ${ }^{256,257}$ the water molecules travel much 
faster than $\mathrm{Au}^{3+}-\mathrm{CTAB}$ micelles during capillary force-assisted vertical diffusion. As water was removed by the absorbent layer, the $\mathrm{Au}^{3+}-\mathrm{CTAB}$ complex concentration was increased in the test zone. It is noteworthy that comparing with the AuNP dissolution without the presence of the absorbent layer, vertical diffusion was significantly enhanced (Figure 28). Therefore, the "coffee ring" reaction pattern was successfully eliminated by the addition of an absorbent layer, which led to a more uniform dissolution with shorter reaction time. The combination of different absorbents, such as paper towel, copy paper, Kimwipe paper, and cotton pad, were tested as well (Figure 28 B-F). It was found that the cotton pad gave a much more uniform pattern after the AuNP dissolution, so it was selected as the absorbent for subsequent studies.

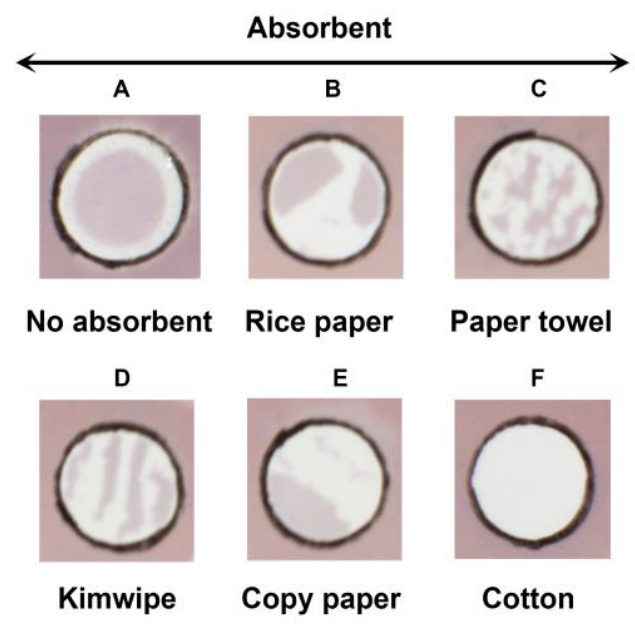

Figure 28. A more even distribution of $\mathrm{Au}^{3+}-\mathrm{CTAB}$ resulted by using an added layer of absorbent

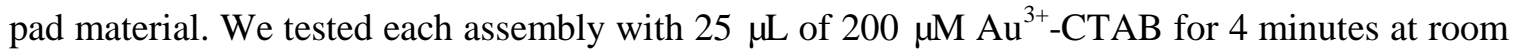
temperature.

\subsubsection{Combination of absorbent layer and AuNP-coated film}

A uniform surface reaction required a perfect match between the AuNP-coated film and the absorbent layer. We found the homogeneity and speed of dissolution could be further improved by employing a "wet-to-wet" combination of wax-circled AuNP-coated film and absorbent cotton layer. The experimental result showed that it took 9 minutes for $25 \mu \mathrm{L}$ of $\mathrm{Au}^{3+}-\mathrm{CTAB}$ solution confined in a dry AuNP-coated test zone to dissolve $80 \%$ of the AuNPs with the presence of a dry 
cotton layer under the AuNP-coated filter paper (Figure 29A), whereas 90\% of the AuNPs were dissolved when the pre-wetted AuNPs-coated film was placed on the top of a dry cotton absorbent layer (Figure 29B). We believe that the non-uniform pattern was formed on the AuNPcoated films, due to the existence of tiny air bubbles between AuNP film and dry cotton layer, which required more time to facilitate mass transport through the film. In contrast, when a tight contact between the pre-wetted AuNP-coated film and cotton layer was formed, all of the AuNPs dissolved after 4 minutes due to uniform mass transportation (Figure 29C).
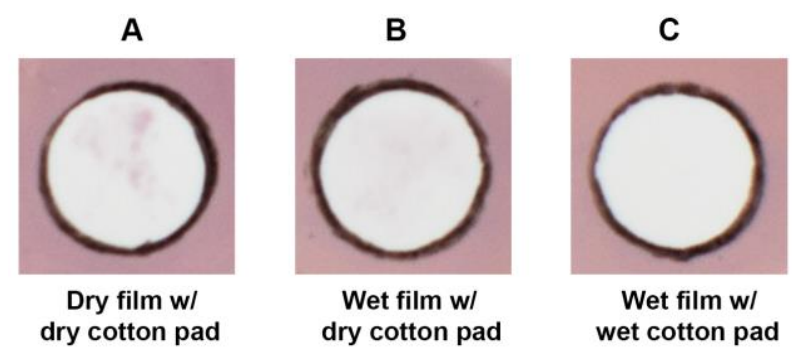

Figure 29. The performance of dry versus wet wax-circled films in conjunction with a dry or wet

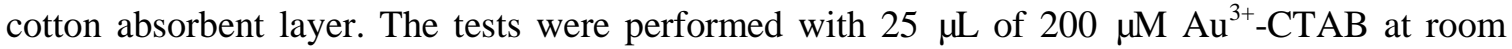
temperature. The intensity of the readouts in the test zone was measured with ImageJ software and AuNP dissolution was calculated by normalizing the intensity of the AuNP film alone to 0\% and the intensity of the MCE filter paper to $100 \%$.

\subsubsection{Fabrication of paper-based device for rapid screening of NADH}

These improvements achieved described above were then successfully incorporated into a simple paper-based device that can uniformly, rapidly detect microliter-scale samples within a surface-confined test zone. The device contains four layers: A) a polyvinyl chloride plastic cover layer $(4.4 \mathrm{~cm} \times 5.4 \mathrm{~cm}, \mathrm{~W} \times \mathrm{L})$ with a $6-\mathrm{mm}$ diameter hole on the top, B) a pre-wetted waxcircled AuNP-coated filter paper, C) a pre-wetted layer of hydrophilic cotton absorbent padding with Kimwipe paper, and A) a bottom plastic cover. All of the four layers were clamped together with paper clips on the edges of the device (Figure 30). 


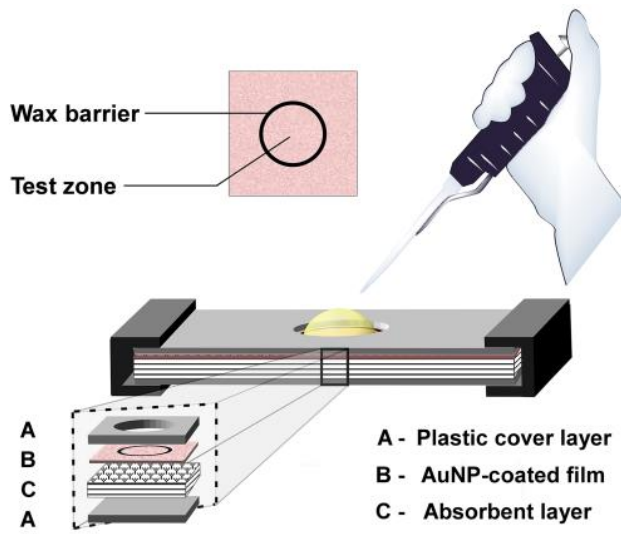

Figure 30. A simple paper-based device for colorimetric detection of NADH in a microliter-scale sample in less than 4 minutes. The device consists of an upper plastic cover layer with a hole exposing the test zone, a wax-circled AuNP-coated paper, a cotton absorbent layer, and a lower plastic cover layer. We added a $25 \mu \mathrm{L}$ reaction solution to the test zone, which is confined within the wax circle on the AuNP-coated paper.

3.3.3.5 Time course on using paper-based device to monitor NADH-mediated AuNP dissolution
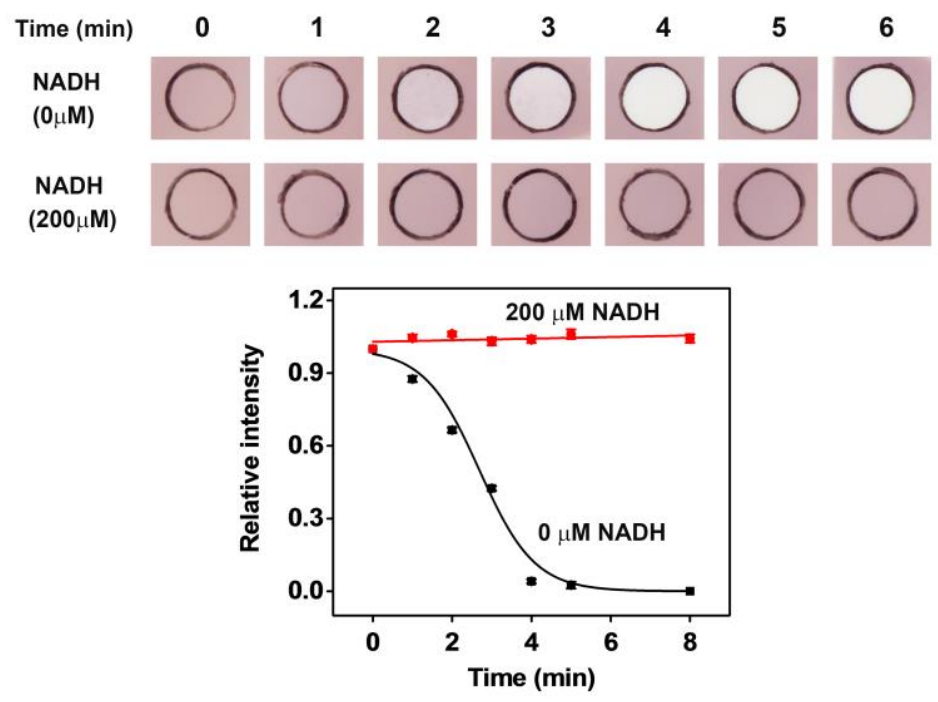

Figure 31. Time course of the reaction in $25 \mu \mathrm{L}$ samples at room temperature on a paper-based device in the absence and presence of $200 \mu \mathrm{M}$ NADH in a $200 \mu \mathrm{M} \mathrm{Au}^{3+}$-CTAB solution.

The time course experiment was performed in the presence of $200 \mu \mathrm{M} \mathrm{Au}{ }^{3+}{ }_{-} \mathrm{CTAB}$ solution with and without the addition of $200 \mu \mathrm{M}$ NADH solution. $25 \mu \mathrm{L}$ of the solution were then added 
to the wax confined test zone at room temperature. The AuNP-coated paper was rinsed with deionized water to remove the extra solution and photographed after the reaction. The results demonstrated that a complete dissolution of AuNPs on this device was obtained after 4 minutes (Figure 31) while AuNP-coated film remained intact in the presence of $200 \mu \mathrm{M}$ NADH.

\subsubsection{Paper-based device to monitor NADH-mediated AuNP dissolution}

To test NADH-mediated inhibition of AuNP dissolution on the paper-based device, $25 \mu \mathrm{L}$ of $200 \mu \mathrm{M} \mathrm{Au}{ }^{3+}-\mathrm{CTAB}$ solution containing different concentrations of NADH were added into the wax circled test zone on the paper-based device. The film was rinsed with deionized water to remove the extra solution and photographed after 4 minutes. As it is shown in the Figure 32A, the presence of NADH could be easily visualized via the developed device at room temperature. Under the optimal condition, as low as $12.5 \mu \mathrm{M}$ of NADH could be clearly visualized on our paper-based device in 4 minutes. The intensity of the color in the test zone could be measured by Image J software and plotted against different amounts of NADH (Figure 32B).

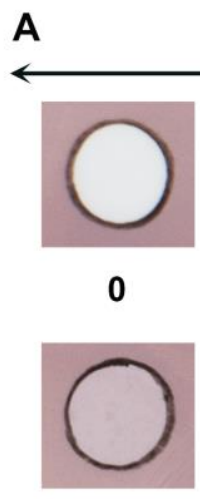

75
NADH $(\mu \mathrm{M})$

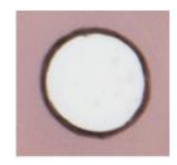

12.5

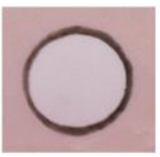

100

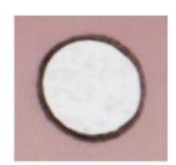

25

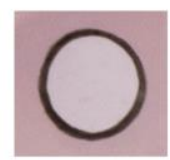

150

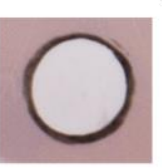

50

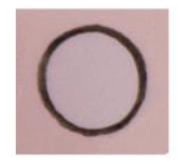

200
B

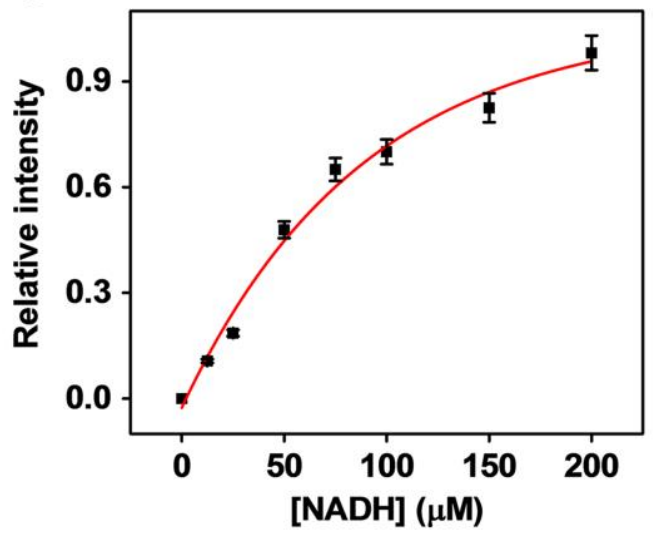

Figure 32. Successful detection of $\mathrm{NADH}$ in $25 \mu \mathrm{L}$ samples in under 4 minutes at room temperature on a paper-based device. (A) We tested multiple concentrations of NADH in $200 \mu \mathrm{M}$ $\mathrm{Au}^{3+}-\mathrm{CTAB}$ solution at room temperature. (B) Test zone readouts were measured with ImageJ software and the relative intensity was calculated by normalizing the unreacted AuNPs-coated film to 1 and the AuNPs-coated film in the absence of NADH to 0 . 


\subsubsection{Using paper-based device to monitor NADH-related enzymatic reaction}

GDH-driven NADH production was also monitored on our paper-based device. We prepared

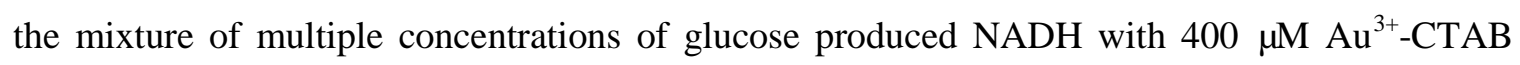
solution and added $25 \mu \mathrm{L}$ of the reaction solution to the wax confined test zone, and measured the intensity with ImageJ software after 4 minutes. The AuNPs in the test zone were completely dissolved in the absence of glucose, indicating that no NADH was being produced. The amount of GDH-produced NADH increased with increasing glucose concentrations. Similar observation was also noticed to the color change of the test zone, which was changed from light pink to red as the glucose concentration increased from 2 to $20 \mathrm{mM}$ (Figure 33). The results confirmed that NADH generated by GDH modulates the dissolution of the AuNP film in our device, generating a colorimetric readout that can be observed within 4 minutes.

A

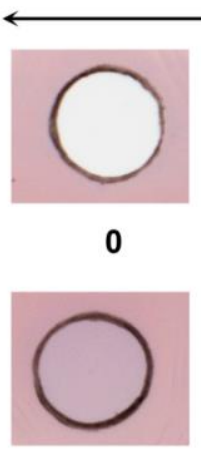

5.5
Glucose (mM)

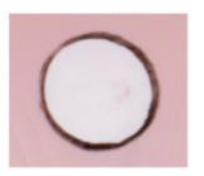

2

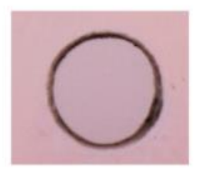

10

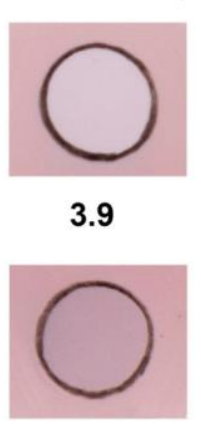

20
B

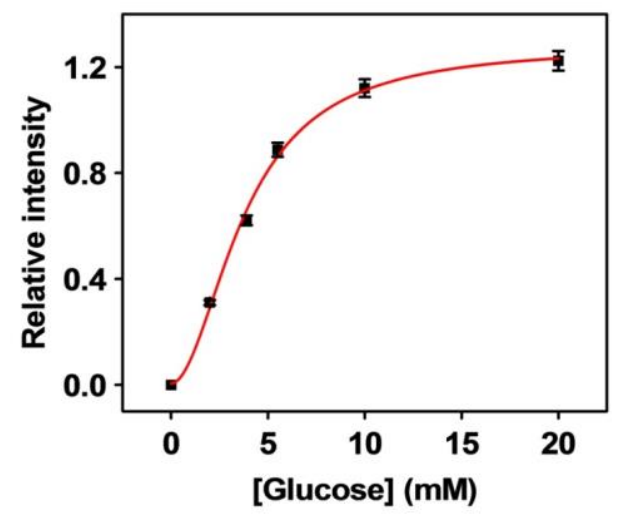

Figure 33. Successful detection of GDH produced NADH in $25 \mu \mathrm{L}$ samples in under 4 minutes at room temperature on a paper-based device. (A) We tested multiple concentrations of glucose produced $\mathrm{NADH}$ in $400 \mu \mathrm{M} \mathrm{Au}{ }^{3+}-\mathrm{CTAB}$ solution at room temperature. (B) Test zone readouts were measured with ImageJ software, and the relative intensity was calculated by normalizing the unreacted AuNPs-coated film to 1 and the AuNPs-coated film in the absence of glucose to 0. 
3.3.3.8 Using paper-based device to monitor NADH and NADH-related enzymatic reaction in complex matrix

To demonstrate the ability of the paper-based device to detect the NADH as well as NAD ${ }^{+}$ driven enzymatic reactions in the complex sample, the detection of NADH in the presence of the E. coli cell lysate was performed. We noted that cell lysate was able to react with the $\mathrm{Au}^{3+}$-CTAB solution due to the reducing reagents existing in the proteins. Therefore, the $\mathrm{Au}^{3+}$-CTAB concentration was increased. Different concentrations of NADH were tested with $200 \mu \mathrm{M} \mathrm{Au}{ }^{3+}$ CTAB in the buffer and $350 \mu \mathrm{M} \mathrm{Au}^{3+}$-CTAB solution in $20 \%$ cell lysate. Test zone readouts with and without cell lysate were measured with ImageJ software, and the relative intensity was calculated by normalizing the unreacted AuNPs coated film to 1 and the AuNPs-coated film in the absence of NADH to 0 (Figure 34). Experimental results showed that this particular complex matrix did not affect the efficiency of our paper-based device for detecting NADH.

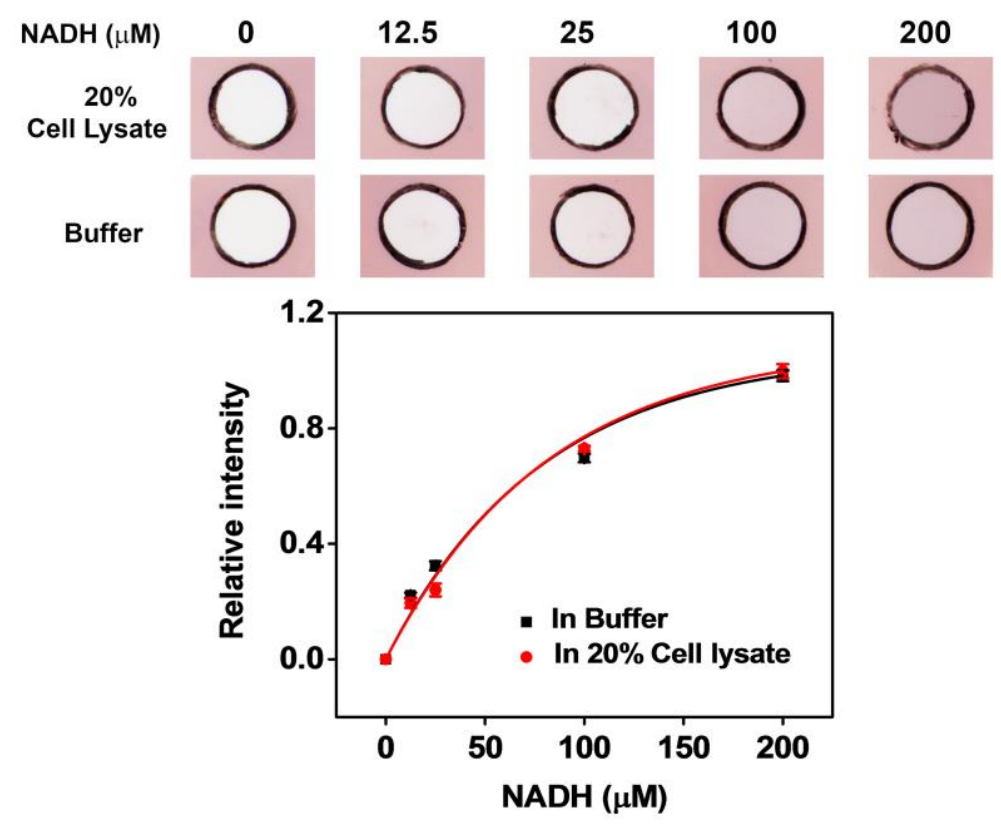

Figure 34. Detecting NADH with and without cell lysate using our paper-based device at room temperature. Multiple concentrations of NADH were tested with and without $20 \%$ cell lysate in $350 \mu \mathrm{M}$ and $200 \mu \mathrm{M} \mathrm{Au}^{3+}$-CTAB solution, respectively. 
As we observed that the cell lysate reacted with $\mathrm{Au}^{3+}-\mathrm{CTAB}$ solution, the $\mathrm{Au}^{3+}-\mathrm{CTAB}$ concentration was increased to $600 \mu \mathrm{M}$ for the sample containing $20 \%$ E. coli cell lysate. In order to test the effect of $E$. coli cell lysate matrix on the NADH-related enzymatic reaction, we prepared the mixture of multiple concentrations of glucose produced NADH with $600 \mu \mathrm{M} \mathrm{Au}{ }^{3+}-$ CTAB solution and added $25 \mu \mathrm{L}$ of the mixture solution to the wax-confined test zone, and measure the intensity with ImageJ software after 4 minutes (Figure 35). Experimental results also showed that this particular complex matrix did not affect the efficiency of our paper-based device for detecting NADH-related enzymatic reaction (Figure 35).

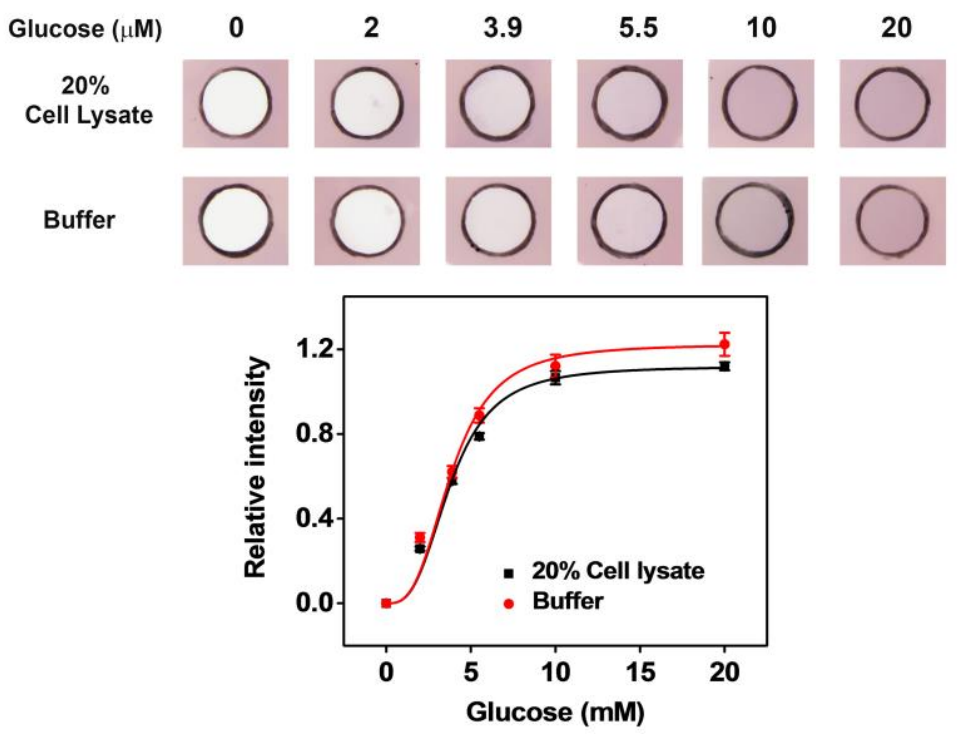

Figure 35. Detecting NADH production by GDH with our paper-based device at room temperature in the absence and presence of $20 \%$ E. coli cell lysate. (A) The device was used to measure NADH production by $0.1 \mathrm{U}$ GDH in the presence of different concentrations $(0-20 \mathrm{mM})$ of glucose in $400 \mu \mathrm{M} \mathrm{Au}^{3+}-\mathrm{CTAB}$ and $600 \mu \mathrm{M} \mathrm{Au}{ }^{3+}-\mathrm{CTAB}$ without and with $20 \%$ E. coli cell lysate after a 4-minute reaction, respectively. (B) The intensity of the readouts in the test zones was measured with ImageJ software, and the relative intensity was calculated by normalizing the unreacted AuNPs film to 1 and the fully dissolved AuNPs-coated film in the absence of glucose to 0 .

\subsubsection{Screening for the GDH inhibitors on our paper-based device}

It is known that heavy metal ions, such as $\mathrm{Ag}(\mathrm{I})$ and $\mathrm{Hg}(\mathrm{II})$, are strong inhibitors of GDH, while there is no inhibitory effect reported for $\mathrm{Pb}(\mathrm{II}) .{ }^{127}$ Before we screened for the inhibitory 
effect of the heavy metal ions on our paper-based device, we first monitored the inhibitory effect of the $\mathrm{Hg}(\mathrm{II})$ on the activity of GDH in the homogenous solution. The kinetic of $\mathrm{Hg}(\mathrm{II})$ inhibitory effect on the NADH production was monitored continually for the first 6 minutes to calculate the enzyme's activity. We defined the enzyme's activity as $100 \%$ in the absence of the $\mathrm{Hg}(\mathrm{II})$ ion. We observed that the activity of GDH decreased as the $\mathrm{Hg}(\mathrm{II})$ concentration increased (Figure 36). The $\mathrm{IC}_{50}$ value is a practical readout of relative effects of different inhibitors on a target enzyme, representing the inhibitor concentration required to inhibit enzyme activity by $50 \%$ under a specific assay condition. As it is shown in Figure 36, the experimental $\mathrm{IC}_{50}$ value for $\mathrm{Hg}(\mathrm{II})$ is $18 \mu \mathrm{M}$.

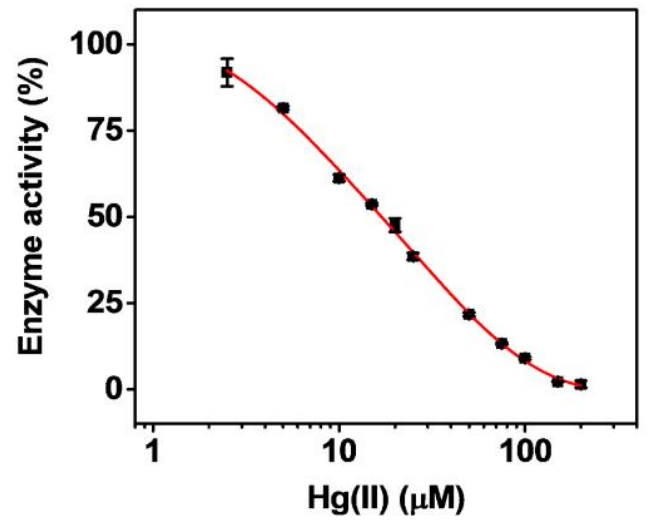

Figure 36. $\mathrm{Hg}(\mathrm{II})$ inhibition effect on GDH activity in homogenous solution. The enzyme's activity was defined as $100 \%$ in the absence of the $\mathrm{Hg}(\mathrm{II})$ ion, and the enzyme's activity decreases as the $\mathrm{Hg}(\mathrm{II})$ concentration increases.

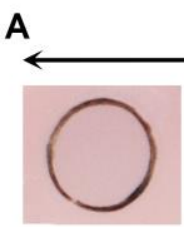

0

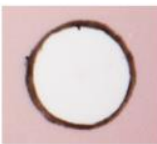

50

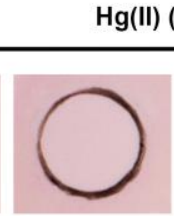

2.5

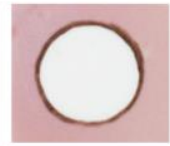

100

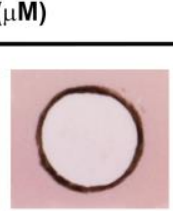

10

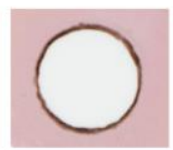

150
B

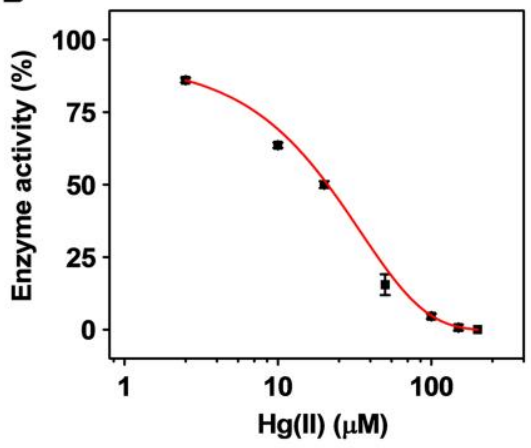

Figure 37. Screening of $\mathrm{Hg}(\mathrm{II})$-mediated inhibition of GDH-driven NADH production at different concentrations. (A) Our paper-based device was used to measure NADH production by $0.1 \mathrm{U}$ GDH in a $25 \mu \mathrm{L}$ sample in the presence of $0-200 \mu \mathrm{M} \mathrm{Hg}$ (II) in $400 \mu \mathrm{M} \mathrm{Au}^{3+}-\mathrm{CTAB}$ after a 
4-minute reaction. (B) The intensity of the readouts in the test zones was measured with ImageJ software and the enzyme activity was calculated by normalizing the unreacted AuNPs film to $100 \%$ and the fully dissolved AuNPs-coated film in the presence of $200 \mu \mathrm{M} \mathrm{Hg}$ (II) to $0 \%$. The calculated $\mathrm{IC}_{50}$ value was $20 \mu \mathrm{M}$.

The inhibitory effects of $\mathrm{Hg}$ (II) were also evaluated by measuring the color intensity produced by GDH enzymatic reaction on the paper-based device. Without $\mathrm{Hg}$ (II) inhibition, $\mathrm{NAD}^{+}$was completely converted into NADH with the assistance of GDH, no AuNP dissolution occurred. Thus, the color of the test zone was in red. The enzyme activity was slightly inhibited by the presence of low concentration of $\operatorname{Hg}$ (II) $(2.5 \mu \mathrm{M})$, so a slight decrease in color intensity in the test zone was observed (Figure 37A). The GDH-produced NADH decreased with the increase of $\mathrm{Hg}(\mathrm{II})$ concentration, which lead to a light pink readout due to partial dissolution of AuNPs. We noted that a completely white readout within the wax-circled test zone was observed in the presence of $100 \mu \mathrm{M} \mathrm{Hg}(\mathrm{II})$, indicating the complete inhibition of GDH and dissolution of AuNPs (Figure 37A). The calculated $\mathrm{IC}_{50}$ value of $20 \mu \mathrm{M}$ for $\mathrm{Hg}(\mathrm{II})$ on the paper-based device is consistent with the value obtained in homogeneous solution $(18 \mu \mathrm{M})$ (Figure 37B).

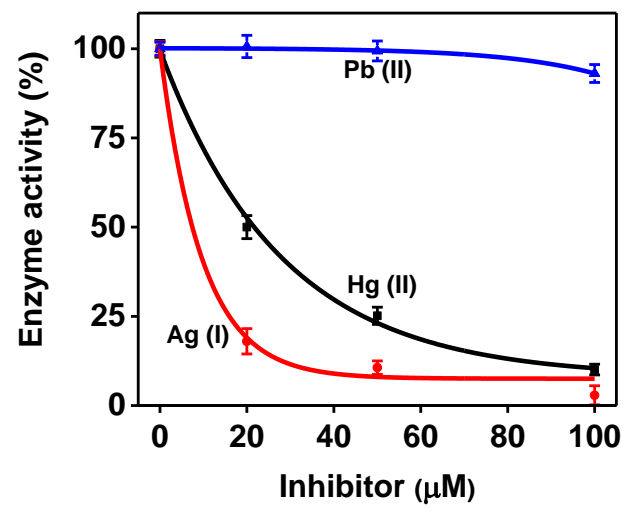

Figure 38. Different ions inhibitory effect on GDH activity in homogenous solution. The enzyme's activity was defined as $100 \%$ in the absence of any metal ion.

To demonstrate the ability of this device to screen for other enzyme inhibitors, we further tested the effects of $\mathrm{Ag}(\mathrm{I})$ and $\mathrm{Pb}(\mathrm{II})$ on GDH-driven $\mathrm{NADH}$ production in the solution by 
monitoring the absorbance of enzyme-generated NADH at $340 \mathrm{~nm}$ for 15 minutes. The enzyme's activity was defined as $100 \%$ in the absence of the heavy metal ions. The experimental results demonstrated that $\mathrm{Ag}(\mathrm{I})$ greatly inhibited the GDH activity, while the $\mathrm{Pb}(\mathrm{II})$ ion almost did not give any inhibition on the enzyme activity (Figure 38). We then tested inhibitory effect of different heavy metal ions on the enzyme activity on the paper-based device in the presence of 20 $\mu \mathrm{M}$ or high $\mathrm{Ag}(\mathrm{I})$, the activity of GDH was completely inhibited and no NADH was generated, yielding a completely white readout (Figure 39). As expected, we did not observe any dissolution of AuNPs in the test zone in the presence of $\mathrm{Pb}$ (II) at concentrations ranging between 20 and 100 $\mu \mathrm{M}$ (Figure 39).This confirmed that $\mathrm{Pb}$ (II) has no inhibitory effect on $\mathrm{GDH}$, which is consistent with the literature. ${ }^{127}$

A

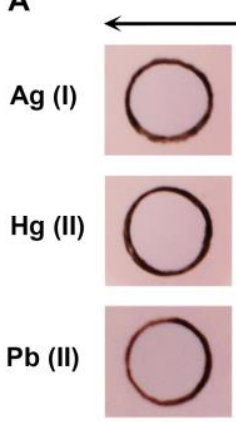

0
Inhibitor $(\mu \mathrm{M})$

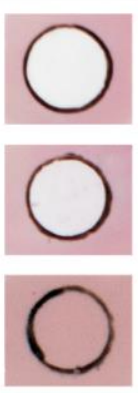

20

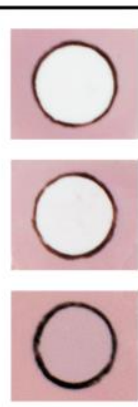

50

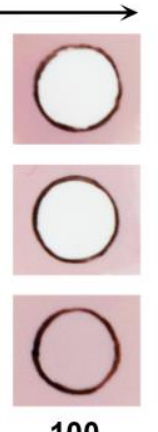

100

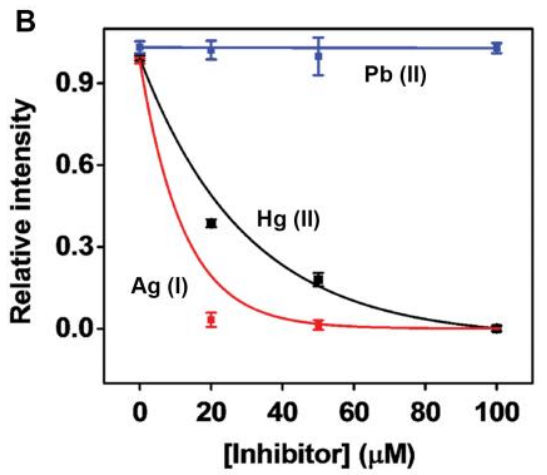

Figure 39. Screening the inhibitory effects of various heavy metal ions on GDH-driven NADH production. (A) We used our paper-based device to measure NADH production by $0.1 \mathrm{U}$ GDH in a $25 \mu \mathrm{L}$ sample containing different concentrations $(0-100 \mu \mathrm{M})$ of heavy metal ions in $400 \mu \mathrm{M}$ $\mathrm{Au}^{3+}-\mathrm{CTAB}$ after a 4 minutes reaction. (B) The intensity of the readouts in the test zones was measured with ImageJ software and the relative intensity was calculated by normalizing the unreacted AuNPs film to 1 and the fully dissolved AuNPs-coated film in the presence of $100 \mu \mathrm{M}$ $\operatorname{Ag}(\mathrm{I})$ to 0 .

\subsection{Conclusion}

A paper-based colorimetric sensing platform/device has been successfully developed for the naked-eye detection of NADH as well as the enzyme-driven NADH production based on the 
dissolution of AuNPs. A simple vacuum filtration technique was employed to prepare a uniform AuNP-coated film on MCE paper. The AuNPs coating was completely dissolved by the $\mathrm{Au}^{3+}-$ CTAB solution without the presence of NADH, resulting in a color change from red to white. In the presence of NADH, $\mathrm{Au}^{3+}$ was quickly reduced to $\mathrm{Au}^{+}$and the dissolution of the AuNP-coated film could be significantly inhibited due to the reduced concentration of $\mathrm{Au}^{3+}$ ion. The colorimetric readout shifts from light pink to red along with the increase of the NADH concentration due to the NADH-reduced AuNPs dissolution. Based on the color change induced by the NADH-inhibited AuNP dissolution, we successfully incorporated the AuNP-coated film into a paper-based device to achieve rapid, simple and efficient detection of NADH with as little as $25 \mu \mathrm{L}$ of sample in 4 minutes at room temperature with very low detection limit $(12.5 \mu \mathrm{M})$. We have further proved that the newly developed platform/device could be employed in a simple and inexpensive manner for the screening of potential dehydrogenase inhibitors. Using GDH as a model system, we further demonstrated that the paper-based device could be used to monitor the $\mathrm{NAD}^{+}$-driven enzymatic reactions in the presence or absence of dehydrogenase inhibitors, e.g., $\operatorname{Ag}(\mathrm{I})$ and $\mathrm{Hg}(\mathrm{II})$. The experimental results confirmed that the reduced production of NADH by heavy metal inhibited GDH increased the dissolution of the AuNPs on the paper-based sensor and generated a colorimetric readout in a $25 \mu \mathrm{L}$ of sample within 4 minutes at room temperature. Although this platform is only a simple proof-of-concept device designed for a single test, it could be easily fabricated with multiple test zones for high throughput testing. We further believe that our device could offer a useful analytical tool for the rapid visualization of other dehydrogenase-mediated $\mathrm{NAD}^{+}$transformations in a variety of contexts. ${ }^{23,104,258-262}$ 


\section{CHAPTER 4: AuNP-based DNA biosensor: A colorimetric sensor based on Exo III-assisted target recycling for single nucleotide polymorphisms (SNP) detection}

\subsection{Introduction}

Single nucleotide polymorphisms (SNPs) happen when a single nucleotide (A, T, C, or G) in the genomic sequence is changed; they have been reported at a rate of approximately once every 1000 bases. SNPs are the most abundant cause of human gene mutation, and multiple SNPs have been found linked to Alzheimer's disease, ${ }^{131}$ Parkinson's disease, ${ }^{132}$ diabetes, ${ }^{133}$ and various cancerous diseases. ${ }^{134}$ SNP detection is therefore important for early diagnosis, clinical prognostics, and disease prevention. ${ }^{135,136}$

Different methods for SNP detection have been widely developed. Sanger sequencing has been considered as a "gold standard" method for DNA analysis. However, it is expensive, laborious, technically demanding, costly, and time-consuming. Enzyme-based detections ${ }^{137-142}$ and hybridization-based methods ${ }^{143-147}$ have been studied for specific and sensitive SNP detection. However, those methods are complicated, usually require expensive instrumentation or high levels of technical expertise, making them impractical for use in small hospital or resourcelimited settings. As such, there remains an urgent need for a simple platform for rapid and sensitive SNP detection in an instrument-free manner. ${ }^{263}$

Colorimetric methods meet most of the above requirements since the resulting color change is readily visualized by the naked eye. The application of AuNPs in DNA detection has been rapidly developed during the last decade due to their surface plasmon resonance absorption that can be changed by the environment, size and physical dimensions. ${ }^{264}$ DNA-AuNP conjugates have been employed as one of the most fascinating nanomaterials for DNA detection. ${ }^{72,152,153}$ Usually, thiolated DNA are modified onto the AuNP surface through gold-sulfur chemistry, ${ }^{265}$ and hundreds of DNA strands can be attached onto a single AuNP. The Mirkin group first reported an AuNP-based SNP detection strategy which relied on hybridization-driven changes in AuNP 
aggregation. In this assay, two sets of DNA-modified AuNPs were cross-linked via target hybridization, and a single-base mismatch was directly observed on a C18 thin-layer chromatography plate at $58{ }^{\circ} \mathrm{C}$. To obtain clinically relevant sensitivity, however, this and subsequently developed AuNP-based SNP detection platforms have required the usage of polymerase chain reaction, ${ }^{157}$ nicking endonuclease, ${ }^{158}$ rolling circle amplification ${ }^{159}$ or real-time ligation chain reaction ${ }^{160}$ to amplify their targets before the detection. Although picomolar concentrations of the target can be successfully detected using these amplified approaches, the target-triggered aggregation of AuNPs requires a reaction time of two hours or longer under precisely controlled temperature. An alternative approach used the color change of unmodified AuNPs to identify SNPs. Based on the differences of electrostatic interaction between single- and double-stranded DNA with unmodified AuNPs, the SNP was easily detected using the red-to-blue color change of unmodified AuNPs within 5 minutes. ${ }^{64}$ However, even for well-optimized conditions, reliable detection with unmodified AuNPs required high target concentration $(>200$ $\mathrm{nM})$ for naked-eye observation. ${ }^{156}$

In response to the above limitations, we presented a simple AuNP-based colorimetric detection method that enabled rapid and sensitive naked-eye detection of SNPs with minimal reagent and equipment requirements at room temperature within 15 minutes. ${ }^{70}$ Specifically, we modified thiolated capture probe onto the AuNP surface and performed SNP detection in a single tube by utilizing Exo III's apurinic endonucleolytic activity to distinguish the perfectly-matched or single-mismatched DNA target. The SNP detection was amplified by target recycling assisted by Exo III's 3' to 5' exonuclease activity. Importantly, there are no specific sequences required for Exo III recognition and digestion, which makes it a good candidate for developing a universal amplification method for SNP detection. 


\subsection{Materials and methods}

Chemicals and materials All DNA sequences were synthesized by Integrated DNA Technologies (USA), purified by HPLC and confirmed by mass spectrometry. Sequences are listed in Table 1. Gold(III) chloride trihydrate, trisodium citrate dihydrate, sodium chloride, magnesium chloride, calcium chloride, Trizma pre-set crystal, potassium acetate, acetate acid were bought from Sigma-Aldrich and used as received. SYBR Gold nucleic acid gel stain $(10,000$ X concentrated in DMSO) was obtained from Invitrogen. Exonuclease III (E. coli) was purchased from New England BioLabs and human apurinic/apyrimidinic endonuclease1 (APE 1) was provided by our collaborator - Dr. Yuan Liu.

Table 1. The employed DNA sequences (from 5' to 3').

Thiolated CP Probe (1) ${ }^{*}$ :

$\mathrm{HST}_{6} \mathrm{ACCACATCATCC/iSpC}_{3} / \mathrm{TATAACTGAAA} / \mathrm{iSpC}_{3} / \mathrm{CCAAACAGT}_{8}$

PM (2): CTGTTTGGCTTTCAGTTATATGGATGATGTGGT

SM-C (3): CTGTTTGGCTTTCAGTTATATCGATGATGTGGT

Unmodified CP probe (4) ${ }^{*}$ :

ACCACATCATCC/iSpC 3 /TATAACTGAAA/iSpC 3 /CCAAACAG T 8

Poly( $\left.\mathbf{T}_{8}\right)$ protected PM (5): CTGTTTGGCTTTCAGTTATATGGATGATGTGGTT 8

Poly $\left(\mathbf{T}_{8}\right)$ protected SM-C (6): CTGTTTGGCTTTCAGTTATATCGATGATGTGGTT 8

SM-T (7): CTGTTTGGCTTTCAGTTATATTGATGATGTGGT

SM-A (8): CTGTTTGGCTTTCAGTTATATAGATGATGTGGT

SM-T (9): CTGTTTGGCTTTCAGTTATTTGGATGATGTGGT

SM-G (10): CTGTTTGGCTTTCAGTTATGTGGATGATGTGGT

${ }^{*} / \mathrm{SpC}_{3} /$ denotes the abasic site (a propyl residue, Spacer- $\mathrm{C}_{3}$ )

AuNPs synthesis and characterization The citrate-coated AuNPs (13 $\pm 1 \mathrm{~nm}$ diameter) were prepared according to the reported literature. ${ }^{266}$ All glasswares were incubated in aqua regia solution $\left(\mathrm{HCl}: \mathrm{HNO}_{3}=3: 1\right.$, v/v) overnight and rinsed thoroughly with tap water followed by deionized water. Gold(III) chloride solution and sodium citrate solution were freshly prepared with deionized water and filtered through a syringe filter $(0.22 \mu \mathrm{m})$. Deionized water $(45 \mathrm{~mL})$ 
was added into three-neck flask incubated in the heating mantle with a stirring bar followed by the addition of $\mathrm{HAuCl}_{4}$ solution $(5 \mathrm{~mL}, 10 \mathrm{mM})$. After the solution was boiling, the sodium citrate solution $(5 \mathrm{~mL}, 38.8 \mathrm{mM})$ was added quickly. The color of the mixture gradually changed from black to wine red in the first two minutes, indicating the formation of AuNPs. The AuNPs solution was kept boiling for another 10 minutes with a stirring speed of $700 \mathrm{rpm}$. After cooling down the solution to room temperature, the AuNP solution was filtered through a syringe filter $(0.22 \mu \mathrm{m})$. The concentration of AuNPs was determined by UV-Vis spectrometer (Cary100, Varian).

Modification of AuNPs with thiolated capture probe The citrate-AuNPs were functionalized with thiolated DNA probe via the well-known gold-thiol chemistry. ${ }^{5}$ All glasswares were incubated with aqua regia overnight and rinsed with deionized water. The glass vials were soaked with $\mathrm{NaOH}(12 \mathrm{M})$ to prevent the adsorption of the AuNPs on the glass wall. The thiolated CP probe DNA (1) was purchased from Integrated DNA Technologies (USA). Since the thiolated DNA strands were received from the company in their oxidized form, the disulfur bond of CP probe was cleaved with freshly prepared Tris(2-carboxyethyl)phosphine hydrochloride (TCEP $-\mathrm{HCl})$ solution $(3 \mathrm{mg} / 100 \mu \mathrm{L})$ for at least 2 hours at room temperature before the modification. ${ }^{267,268}$ The reduced thiolated DNA was slowly added into freshly prepared citrate-capped AuNPs $(3 \mathrm{~mL})$ under the stirring at room temperature. The resulting solution was covered with aluminum foil and continuously stirred $(700 \mathrm{rpm})$ at room temperature. After the DNA modification for 12 hours, the $\mathrm{NaCl}$ solution $(4 \mathrm{M}, 50 \mu \mathrm{L})$ was gradually added into the reaction mixture with a maximum stirring speed. After stirring (700 rpm) for another 12 hours, the $\mathrm{NaCl}$ solution $(4 \mathrm{M}, 100 \mu \mathrm{L})$ was added to the solution with a maximum stirring speed. The same salt aging step was repeated after a 6-hour incubation at room temperature. The final concentration of $\mathrm{NaCl}$ is $\sim 0.3 \mathrm{M}$. The solution was continuously stirred for another 12 hours. The DNA-modified AuNPs were separated from the supernatant using a centrifugal filter (100 KD) at 
$1500 \mathrm{rcf}, 4{ }^{\circ} \mathrm{C}$ for 20 minutes, forming a red pellet of AuNPs at the bottom. The supernatant was collected into a Corning tube for DNA concentration measurement. The particles were resuspended in $1 \mathrm{~mL}$ Trizma buffer (10mM, pH 7.4) and centrifuged (1500 rcf) at $4{ }^{\circ} \mathrm{C}$ for $10 \mathrm{~min}$ for six times. Finally, the AuNP were re-suspended in $300 \mu \mathrm{L}$ of Trizma buffer (10 mM, pH 7.4) and stored at $4{ }^{\circ} \mathrm{C}$.

Colorimetric detection of SNP The reaction buffer is $20 \mathrm{mM}$ Tris-acetate buffer (pH 7.9) including $50 \mathrm{mM} \mathrm{KAc}, 25 \mathrm{mM} \mathrm{NaCl}, 20 \mathrm{mM} \mathrm{CaCl}_{2}$ and $3 \mathrm{mM} \mathrm{MgCl}_{2}$. The colorimetric detection of SNP was performed in $100 \mu \mathrm{L}$ of reaction buffer with $300 \mathrm{nM} \mathrm{CP}$-modified AuNPs and $50 \mathrm{U}$ Exonuclease III, with or without $20 \mathrm{nM}$ PM (perfectly-match) (2) or SM (single-mismatched) (3) DNA at room temperature. The color change was observed by naked eye and further confirmed in a microplate reader (Tecan Infinite M1000 Pro). The DF is defined as the ratio of the net signal gain (after subtracting background from probe only) obtained with the PM target to that obtained with the SM target.

To test the effect of human apurinic/apyrimidinic endonuclease 1 (APE1) on the sensor performance, the reaction was performed under the same buffer condition with $100 \mathrm{nM}$ APE 1 , and the UV-Vis spectra of the CP-modified AuNPs was monitored either alone or in the presence of $20 \mathrm{nM}$ PM or SM. The influence of $\mathrm{Ca}^{2+}$ concentration on target discrimination and detection time was performed in the $20 \mathrm{mM}$ Tris-acetate ( $\mathrm{pH}$ 7.9) with $50 \mathrm{mM} \mathrm{KAc}, 25 \mathrm{mM} \mathrm{NaCl}$ and 3 $\mathrm{mM} \mathrm{MgCl}$, with variable concentrations of $\mathrm{Ca}^{2+} .50 \mathrm{U}$ of Exonuclease III, $300 \mathrm{nM} \mathrm{CP}$-modified AuNPs and $20 \mathrm{nM}$ PM or SM were used in the reaction. Similarly, the influence of $\mathrm{Mg}^{2+}$ concentration was performed in the reaction buffer containing $20 \mathrm{mM}$ Tris-acetate (pH 7.9), 50 $\mathrm{mM} \mathrm{KAc}, 25 \mathrm{mM} \mathrm{NaCl}$ and $20 \mathrm{mM} \mathrm{CaCl}_{2}$, with variable concentrations of $\mathrm{Mg}^{2+} .50 \mathrm{U}$ of Exo III, 300 nM CP-modified AuNPs, and 20 nM PM or SM were also used in the reaction.

The kinetic experiments were carried out in the $20 \mathrm{mM}$ Tris-acetate buffer ( $\mathrm{pH} 7.9)$ with 50 $\mathrm{mM} \mathrm{KAc}, 25 \mathrm{mM} \mathrm{NaCl}, 20 \mathrm{mM} \mathrm{CaCl} 2$ and $3 \mathrm{mM} \mathrm{MgCl}_{2}$. The colorimetric detection of DNA 
was performed in $100 \mu \mathrm{L}$ of reaction buffer with $300 \mathrm{nM} \mathrm{CP}$-modified AuNPs and $50 \mathrm{U}$ Exonuclease III, with different PM target concentrations at room temperature. To test the detection limit, the PM or SM targets were incubated with the CP-modified AuNPs at $4{ }^{\circ} \mathrm{C}$ for 4 hours and the color change was monitored .

Polyacrylamide gel electrophoresis experiment Reaction mixtures were prepared in the reaction buffer with $2 \mu \mathrm{M}$ unmodified $\mathrm{CP}$ probe (4), either alone or with $200 \mathrm{nM}$ poly $\left(\mathrm{T}_{8}\right)$ protected PM (5) or SM-C (6) target. The reaction mixtures were challenged with $10 \mathrm{U}$ Exo III for 1 minute or $100 \mathrm{nM} \mathrm{APE} 1$ for 10 minutes at room temperature and then stopped by heatinactivation at $80{ }^{\circ} \mathrm{C}$ for 10 minutes. Five $\mu \mathrm{L}$ aliquots of the reaction mixtures were subjected to electrophoresis on a $12 \%$ denaturing polyacrylamide gel $(17 \times 15 \mathrm{~cm})$ containing $20 \mathrm{mM}$ Trisacetate buffer ( $\mathrm{pH} 7.0$ ) with $0.5 \mathrm{mM}$ EDTA at $15 \mathrm{~V} / \mathrm{cm}$ for 3 hours at room temperature. The gel was stained for 20 minutes with $1 \times$ SYBR Gold and imaged using the ChemiDoc MP imaging system (Bio-Rad).

\subsection{Results and discussions}

\subsubsection{AuNP characterization}
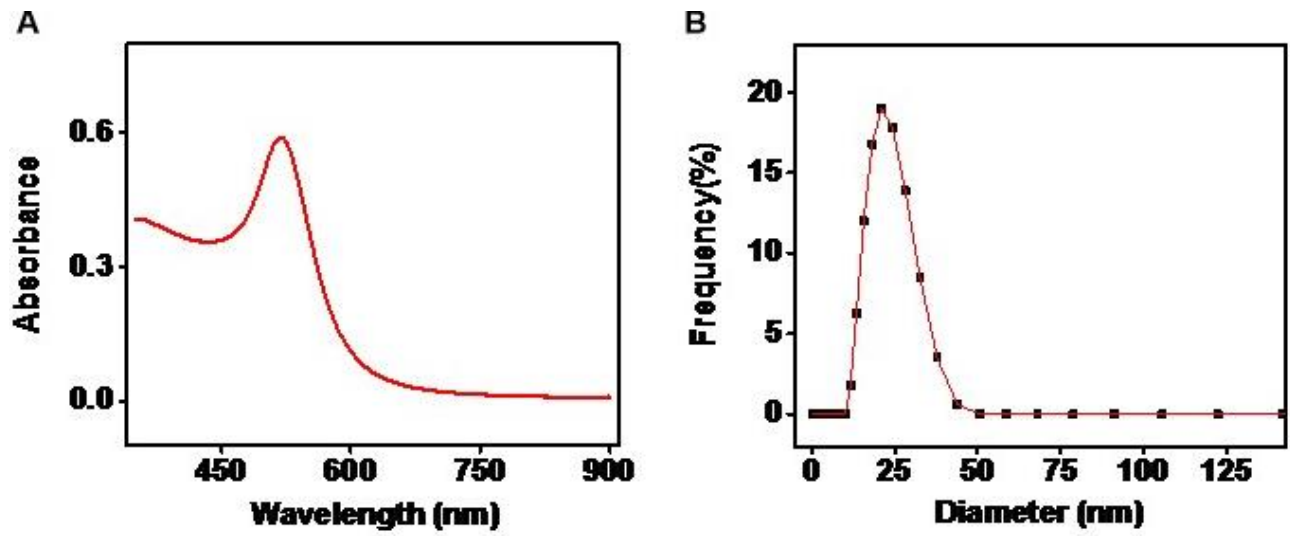

Figure 40. Characterization of the concentration and size of these synthesized AuNPs. (A) UVVis spectrum of the AuNPs with a maximum wavelength of $519 \mathrm{~nm}$. (B) Size distribution measurement using Zetasizer: the average size of the AuNP is $21 \mathrm{~nm}$, and the polydispersity index (PDI) is 0.1 . 
The citrate-capped AuNPs (13 $\pm 1 \mathrm{~nm}$ diameter) were synthesized using a modified version of Turkevich's method. ${ }^{266}$ The maximum wavelength of the freshly-prepared AuNPs is $519 \mathrm{~nm}$ (Figure 40A). The AuNPs concentration is $10.9 \mathrm{nM}$, which is calculated by using Beer's Law $(\mathrm{A}=\mathrm{Ebc})\left(\mathcal{E}=2.7 \times 10^{8} \mathrm{~cm}^{-1} \mathrm{M}^{-1}\right){ }^{269}$ The hydrodynamic size distribution and the polydispersity index (PDI) were measured using the Malvern dynamic light scattering (DLS) Zetasizer Nano (Malvern. UK). The average size of the AuNP is $21 \mathrm{~nm}$ and the PDI is 0.10 , which indicates a narrow size distribution (Figure 40B). We further measured the actual size of the AuNPs with transmission electron microscopy (TEM) (Phillips CM-200) under $200 \mathrm{kV}$ on the regular copper grid covered with carbon film. According to the TEM image, we determined the size distribution of the AuNPs by counting at least 200 particles and obtained a very narrow size distribution with an average size of $13.5 \pm 1 \mathrm{~nm}$ (Figure 41 ).
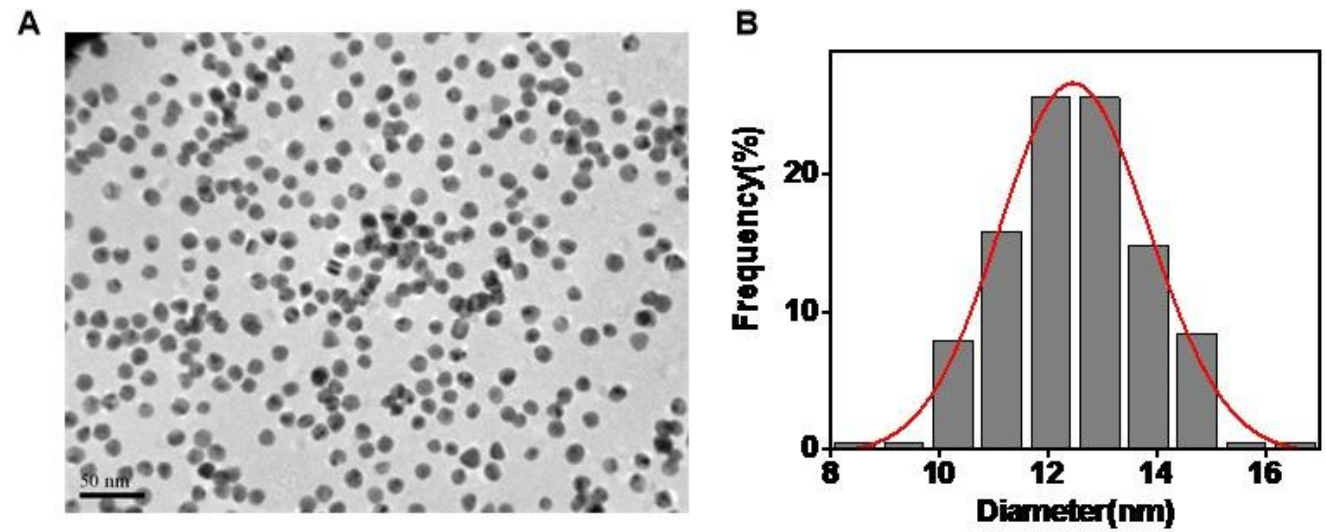

Figure 41. Size characterization of the synthesized AuNPs. (A) TEM image of the AuNPs; (B) Size distribution of the AuNPs.

The absorbance spectrum of the CP-modified AuNP was measured by a UV-Vis spectrometer before and after modification, with both spectra normalized to 1 OD. The absorbance at $519 \mathrm{~nm}$ and the absorbance difference at $260 \mathrm{~nm}$ were recorded to calculate the numbers of DNA modified on the AuNPs. The DNA-modified AuNPs are highly stable in salt solutions due to the strong electrostatic repulsion. On the basis of the extinction coefficient of both the DNA strand at $260 \mathrm{~nm}$ and AuNPs at $519 \mathrm{~nm}$, we obtained a molar ratio of $\sim 13 \mathrm{CP}$ probes per AuNP. 


\subsubsection{Probe design}

The sequence of the capture probe is designed as 5 '-thiol-poly $\left(\mathrm{T}_{6}\right)-(\mathrm{N})_{11}-\left(5^{\prime}-\mathrm{CXT}\right)-(\mathrm{N})_{9}-$ $3^{\prime}\left(\mathrm{AXC}-3^{\prime}\right)-(\mathrm{N})_{7}-$ poly $\left(\mathrm{T}_{8}\right)-3^{\prime}$ (Figure $\left.42 \mathrm{~A}\right)$. The $5^{\prime}$ poly $\left(\mathrm{T}_{6}\right)$ acts as a flexible linker that extends the active recognition portion of the oligonucleotide sequence away from the AuNP surface to improve target hybridization, and the $3^{\prime}$ poly $\left(\mathrm{T}_{8}\right)$ serves as a sticky end to protect the $\mathrm{CP}$ from 3' to 5' exonucleolytic digestion by Exo III. Two nucleotides were intentionally replaced with two C3 spacers (propanediol) to form two abasic sites (X, 5'and 3' abasic site) within CP probe for creating active sites for Exo III's AP incision. ${ }^{270}$ This capture probe is specifically designed to recognize a target DNA sequence in which the SNP site is situated directly to the 5 ' abasic site to discriminate SNP. The 3' abasic site is used for Exo's incision/digestion in order to easily facilitate target release at room temperature.

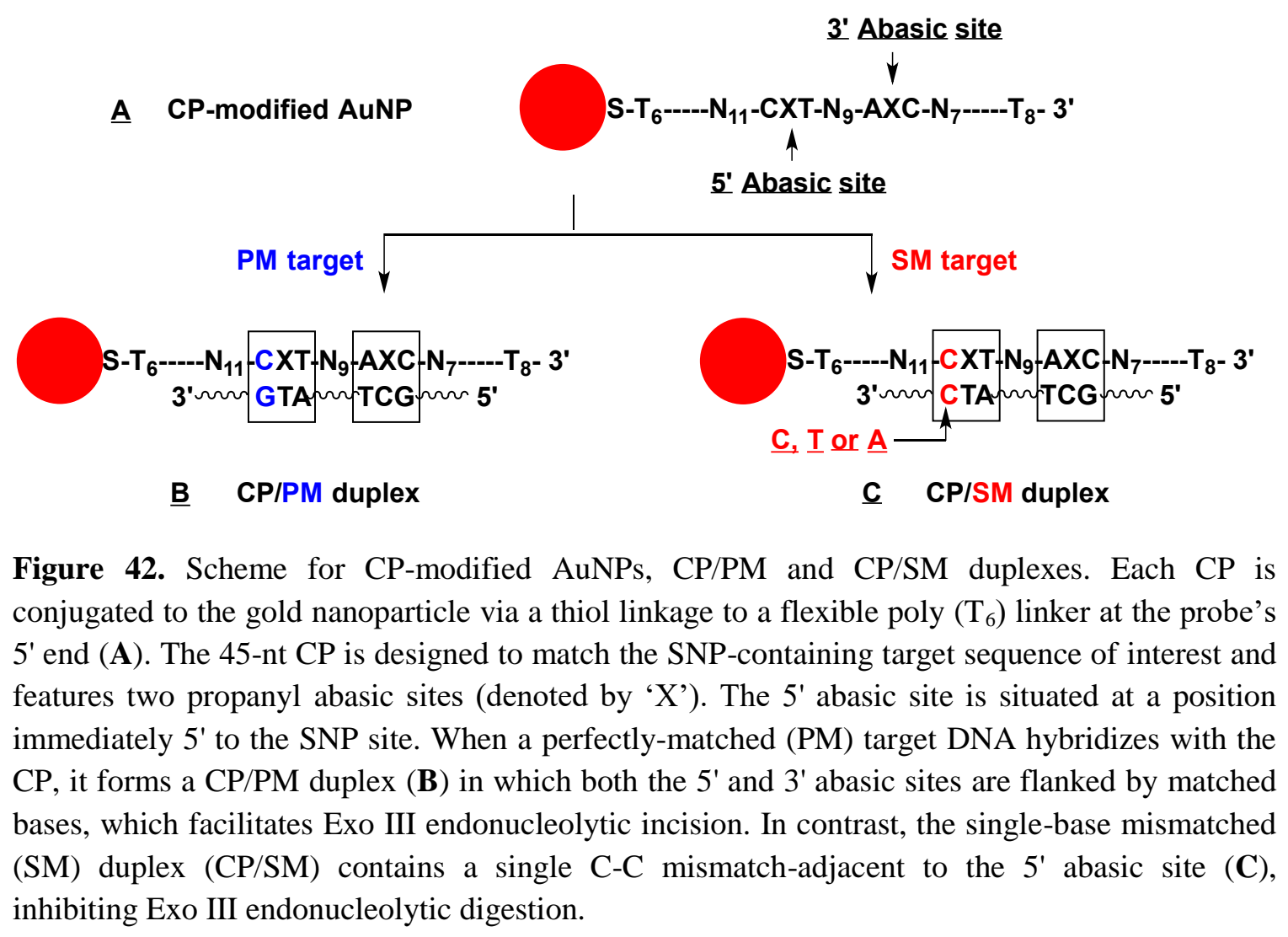


In the absence of the target DNA, the CP-modified AuNPs are homogeneously dispersed in the high salt solution due to the DNA protection on the surface and maintain a red color In the presence of the PM target, both the 5' and 3' abasic sites are flanked by matched bases, which facilitates Exo III endonucleolytic incision and 3' to 5' exonuclease activity (Figure 42B, CP/PM duplex). Then Exo III further digests capture probe to shorter DNA and causes a color change. In the case of SM target (Figure 42C, CP/SM duplex), Exo III endonucleolytic digestion at 5' abasic sites is inhibited, and the modified AuNPs fail to aggregate and remains red. SNP discrimination is achieved by differentiating Exo's AP activity performed on the PM or SM duplexes, and the colorimetric signal reporting is achieved through the active $3^{\prime}$ to $5^{\prime}$ digestion of Exo III on the cleaved capture probe.

\subsubsection{Colorimetric detection of SNP}

Colorimetric detection of SNP is performed in a single tube with a set of DNA probemodified AuNPs, Exo III, and target DNA. The CP-modified AuNPs are homogeneously dispersed in high-salt reaction buffer due to protection by the immobilized CP strands (Figure 43A). When the 33-nt PM DNA hybridizes with CP-modified AuNPs in the solution, both the 5' and $3^{\prime}$ abasic sites are flanked by matched bases, with a 3' poly(T8) sequence of CP forming a sticky end (Figure 43B). Exo III preferentially cleaves the match-flanked abasic sites through its apurinic endonucleolytic activity (Figure 43C) because the $3^{\prime}$ to $5^{\prime}$ exonucleolytic activity is inactive at the sticky end with poly $\left(\mathrm{T}_{8}\right)$ protection, ${ }^{271}$ converting the $\mathrm{CP}$ into three nicked duplex fragments. Exo III then recognizes the newly-formed 3'-hydroxyl termini on the nicked ends of the $\mathrm{CP}$ fragments and rapidly catalyzes the stepwise removal of 5'-mononucleotides through its exonucleolytic activity (Figure 43D), releasing the PM strand intact from the particle (Figure 43E). The released PM target hybridizes with another CP on the same or a different AuNPs to begin the cycle anew (Figure 43F). Ultimately, all of the CPs are sheared from the AuNPs, leaving only short poly $\left(\mathrm{T}_{6}\right)$ linkers behind on the particles. The sheared AuNPs are no longer 
stable in the presence of high concentration salt reaction buffer due to the loss of the protection from negatively charged DNA and undergo salt-induced aggregation (Figure 43G), giving rise to a red-to-blue color change. Since single target can be recycled for many CP-modified AuNPs, the SNP signal is rapidly amplified with visible AuNP aggregation within 15 minutes.

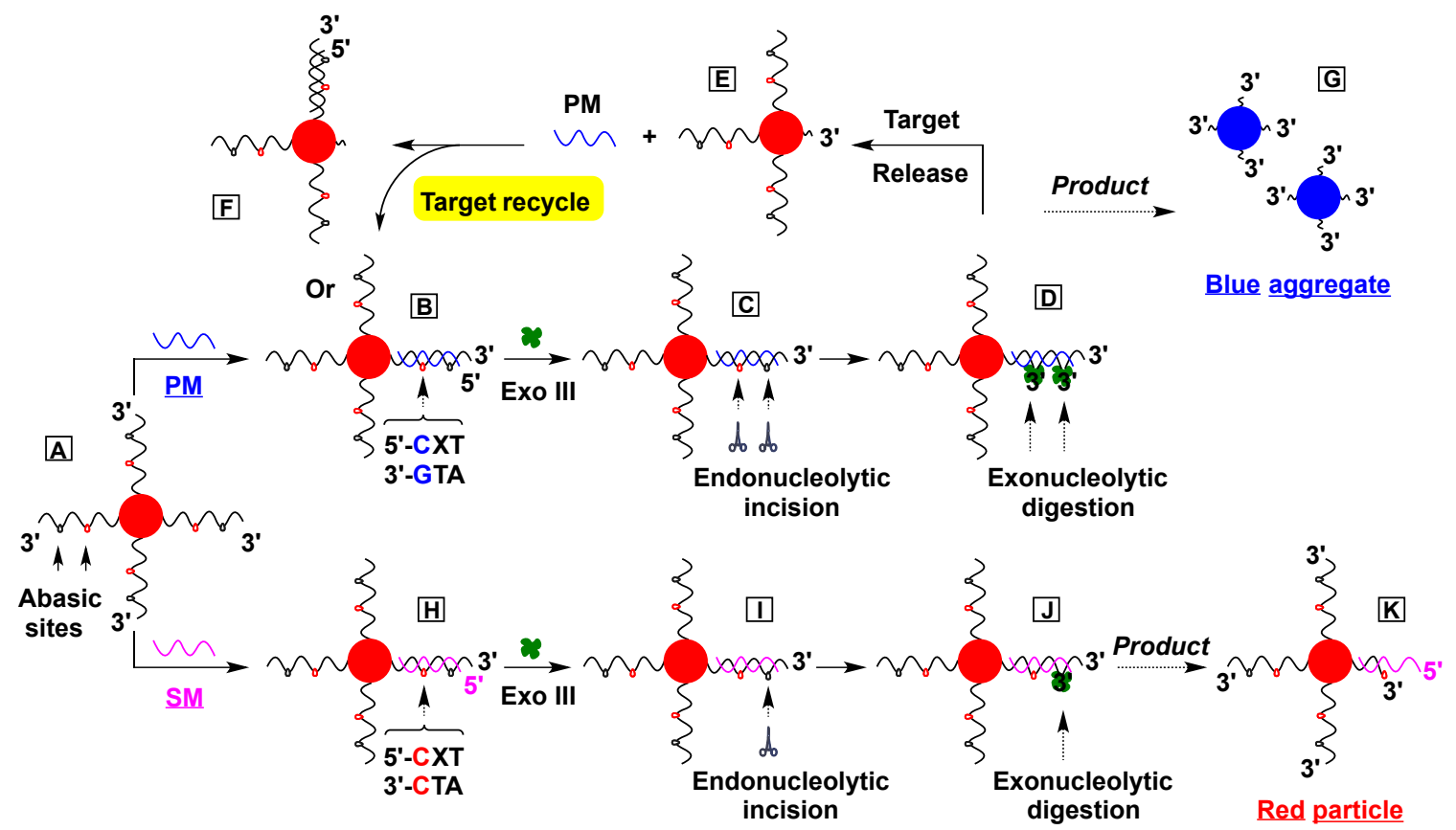

Figure 43. Scheme for AuNP-based, Exo III-amplified colorimetric SNP detection. (A) The CPmodified AuNPs are homogeneously dispersed in the buffer. (B) PM target hybridization with CP forms a perfectly matched duplex with a $3^{\prime}$ poly $\left(\mathrm{T}_{8}\right)$ sticky end. (C) Exo III preferentially cleaves both match-flanked abasic sites through its apurinic endonucleolytic activity, generating two nicks. (D) Exo III recognizes the 3'-hydroxyl termini on these nicked ends and rapidly digests these fragments via its exonucleolytic activity. (E) The CP fragments are sheared from the particle, releasing the intact PM target. (F) The PM target is recycled, hybridizing with a new CP on the same or a different AuNPs. (G) In the absence of CP strands, the AuNPs undergo saltinduced aggregation with an associated red-to-blue color change. (H) In contrast, a single mismatch flanking the 5' abasic site in the CP-SM duplex inhibits the apurinic endonucleolytic activity of Exo III. (I) Exo III therefore only cleaves the match-flanked 3' abasic site, generating a single nick. (J) The enzyme subsequently performs 3'-to-5' exonucleolytic digestion. (K) DNA digestion dramatically slows down as Exo III approaches the mismatch-flanked 5' abasic site, leaving behind DNA duplex fragments on the particle. These fragments prevent salt-induced AuNPs aggregation and the solution remains red. 
When the CP-modified AuNPs hybridize with 33-nt SM DNA targets, the C-C mismatchflanked 5' abasic site (5'-CXT/CTA) (Figure 43H) significantly inhibits the apurinic endonucleolytic activity of Exo III. ${ }^{272}$ Exo III therefore preferentially only cleaves the matchflanked 3' abasic site, generating two nicked CP duplex fragments (23- and 8-bp) (Figure 43I). The enzyme subsequently catalyzes exonucleolytic digestion at this site (Figure 43J). However, this digestion dramatically slows down as Exo III approaches the mismatch-flanked 5' abasic site ${ }^{273}$ leaving DNA duplex fragments $(\geq 12-\mathrm{bp})$ intact on the surface of the particle. These long DNA duplexes have a melting point of $\geq 46.4{ }^{\circ} \mathrm{C}$, limiting target release at room temperature and thus inhibiting target recycling. These undigested fragments and probes protect the AuNPs against salt-induced aggregation (Figure 43K). The AuNP solution will stay red colored because of the long DNA sequence stabilizing the AuNPs.

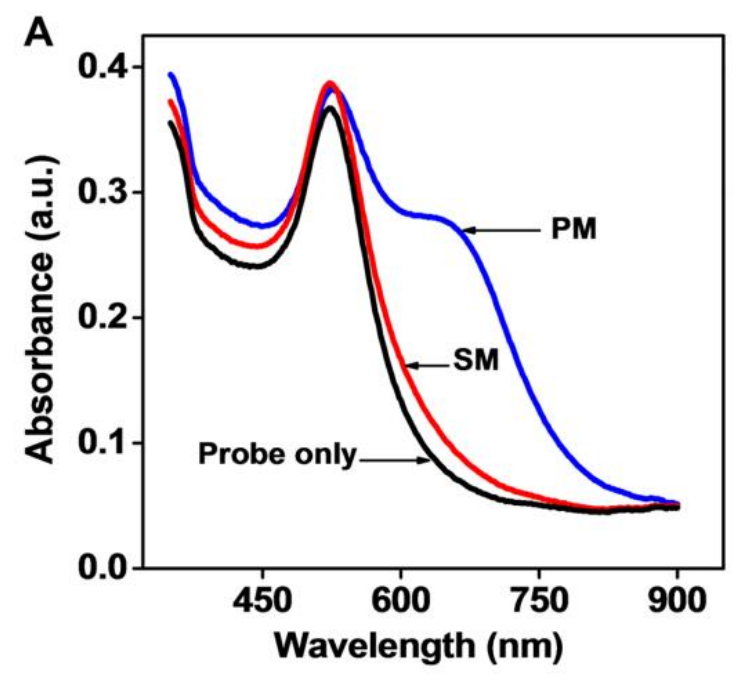

B

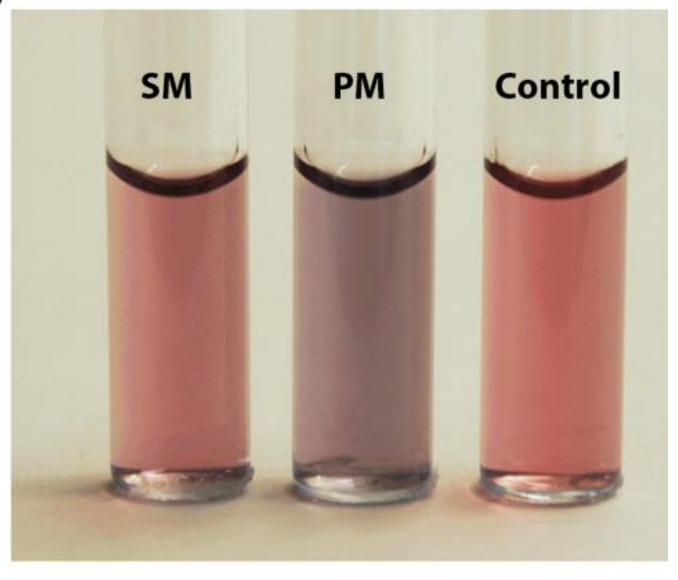

Figure 44. AuNP-based, Exo III-amplified colorimetric SNP detection within 15 minutes at room temperature. (A) UV-vis spectra of reactions performed in the presence of 50 U Exo III with CPmodified AuNPs alone (probe only) or with $20 \mathrm{nM} \mathrm{SM}$ or PM. (B) A red-to-blue color change is apparent in the PM sample relative to the SM sample or probe only (control).

The colorimetric detection of SNP was simply performed in $100 \mu \mathrm{L}$ of Tris-acetate reaction buffer $(20 \mathrm{mM}, \mathrm{pH} 7.9)$ containing $\mathrm{KAc}(50 \mathrm{mM}), \mathrm{NaCl}(25 \mathrm{mM}), \mathrm{CaCl}_{2}(20 \mathrm{mM}), \mathrm{MgCl}_{2}(3$ 
mM), 300 nM CP-modified AuNPs and 50 U Exo III, with or without PM (20 nM) or SM (20 nM) at room temperature. The color change was successfully observed by the naked eye; the solution with the CP-modified AuNPs and the PM DNA target turned blue within 20 minutes due to aggregation of the sheared AuNPs in the high concentration of salt. In contrast, these immobilized CPs were highly stable and resistant to the 3 '-to-5' exonucleolytic activity of Exo III in the presence of the SM target. The solution stayed red, and no color change was observed within 2 hours (Figures 44B). The result was further confirmed in the microplate reader (Figures 44A).

\subsubsection{Human apurinic/apyrimidinic endonuclease 1 (APE 1)}

To perform SNP detection with salt-induced AuNP aggregation, the apurinic endonucleolytic and 3' to 5' exonuclease activities of Exo III need to work synergistically. Control experiment was performed to confirm that exonucleolytic digestion of Exo III is absolutely necessary to achieve aggregation of CP-modified AuNPs. Human apurinic/apyrimidinic endonuclease 1 (APE 1) is known to hydrolyze the phosphodiester backbone specifically at the $5^{\prime}$ end of an abasic site, creating a nick in the DNA duplex and leaving a 1-nt gapped intermediate with 3'-hydroxyl and 5'-deoxyribose phosphate termini. ${ }^{274}$ APE 1 was added into the detection platform to replace the Exo III. However, APE 1 is known to possess no exonucleolytic activity. We did not observe any red-to-blue color change of CP-modified AuNPs either with the PM or SM (Figure 45). All the solution remained red even after 4 hours in the presence of $100 \mathrm{nM}$ APE 1, which indicated that the CP fragments remain bound to the AuNPs in the absence of 3 '-to-5' exonucleolytic activity and protected the AuNPs from salt-induced aggregation. These results clearly proved the important role of the Exo III exonucleolytic activity in forming a red-to-blue aggregation of AuNPs in order to discriminate between PM and SM. 


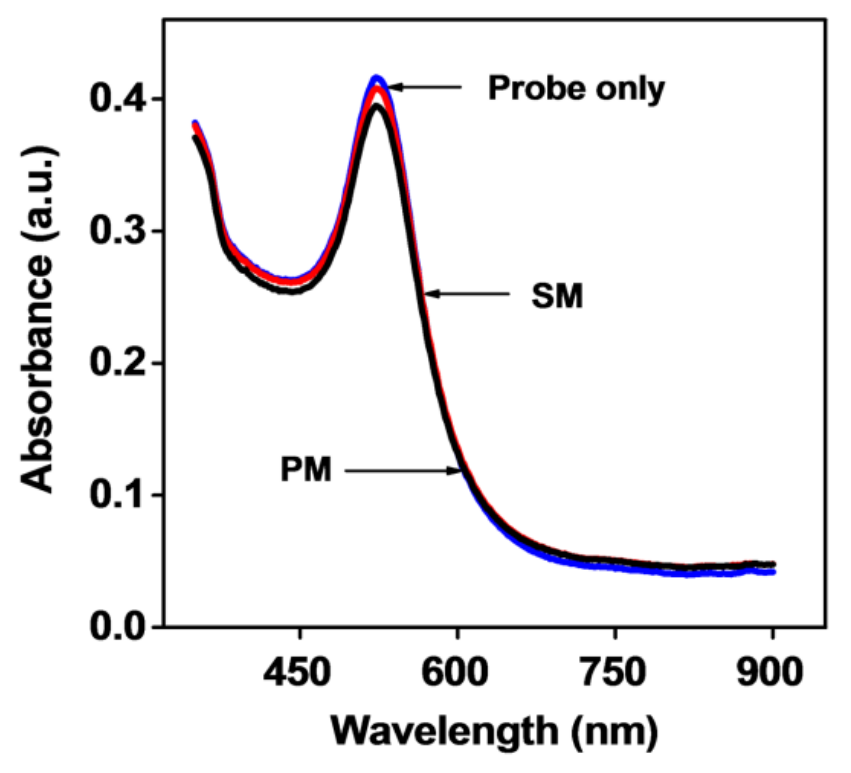

Figure 45. CP-modified AuNPs remained the protection against salt-induced aggregation even after 4-hour treatment with APE 1, which lacks exonuclease activity. UV-vis spectra of the modified AuNPs alone or with $20 \mathrm{nM}$ PM or SM in the presence of $100 \mathrm{nM}$ APE 1 are represented.

\subsubsection{Role of Exo III activities}

To confirm the contribution of the exonucleolytic activity of Exo III to our AuNP-based SNP detection, $2 \mu \mathrm{M}$ unmodified $\mathrm{CP}$ probes $(4,39-n t)$ without 5 ' poly $\left(\mathrm{T}_{6}\right)$ ends were digested with Exo III or APE 1 in the presence of $200 \mathrm{nM} \mathrm{PM} \mathrm{(5,} \mathrm{41-nt)} \mathrm{or} \mathrm{SM} \mathrm{(6,} \mathrm{41-nt)} \mathrm{targets} \mathrm{in} \mathrm{the} \mathrm{reaction}$ buffer. The PM and SM targets were protected from the enzymatic digestion by a poly $\left(\mathrm{T}_{8}\right)$ at $3^{\prime}$ end. The reaction mixtures were challenged with 10 U Exo III for 1 minute or $100 \mathrm{nM}$ APE 1 for 10 minutes at room temperature, then heat at $80^{\circ} \mathrm{C}$ for 10 minutes to inactivate the activity of the enzyme. The aliquots of the reaction mixtures were subjected to electrophoresis on a $12 \%$ denaturing polyacrylamide gel containing $20 \mathrm{mM}$ Tris-acetate buffer $(\mathrm{pH} 7.0)$ with $0.5 \mathrm{mM}$ EDTA at room temperature. The gel was stained for 20 min with $1 \times$ SYBR Gold and imaged with the ChemiDoc MP imaging system (Bio-Rad). 
Gel electrophoresis data demonstrated that both Exo III and APE 1 were able to discriminate a single mismatch at the SNP site due to their apurinic endonucleolytic activity (Figure 46). All three major products from enzymatic cleavage (27-nt, 23-nt and 16-nt) (Figure 46A) were clearly visualized on the gel, although in varying amounts that reflected differences in processing between different enzyme-duplex combinations (Figure 46B). When the unmodified capture probe was digested with Exo III, the matched 3' abasic site was efficiently incised for both duplexes, resulting in the formation of 16-nt and 23-nt fragments regardless of PM or SM (Figure 46A). We observed 12-nt and 27-nt products in the CP/PM sample due to the efficient endonucleolytic incision at the matched 5' abasic site, while such products were barely observed in the mismatch-containing CP/SM sample (Figure 46B). The Exo III could then recognize the nicked $3^{\prime}$ ends and digest the incised CP fragments (Figure 46A) using 3 ' to 5' exonuclease activities. The intact target was recycled, and most of the 39-nt probes were depleted in the PM sample, and only a tiny amount of 27 - and 23 -nt products remained after this reaction (Figure 46B, PM with Exo III). Since Exo III was inactivated on the mismatched 5' abasic site for the CP/SM duplex, only higher enzymatic efficiency at its matched 3' abasic site was observed (Figure 46B, 23-nt product).

In contrast, the exonucleolytic activity of Exo III was significantly inhibited on the CP/SM duplexes due to the inactivation of Exo III on the mismatch-flanked 5' abasic site, inhibiting target release and thus preventing target recycling. Therefore, a large number of the probe remained intact (Figure 46B, SM with Exo III). We observed a smeared band of $\sim 23$-nt product in the $\mathrm{CP} / \mathrm{SM}$ samples, indicating that the initial 23-nt product was degraded to various smaller products by Exo III exonucleolytic digestion. No exonucleolytic digestion of Exo III occurred on the poly $\left(\mathrm{T}_{8}\right)$ related 16-nt incised product in both samples. 


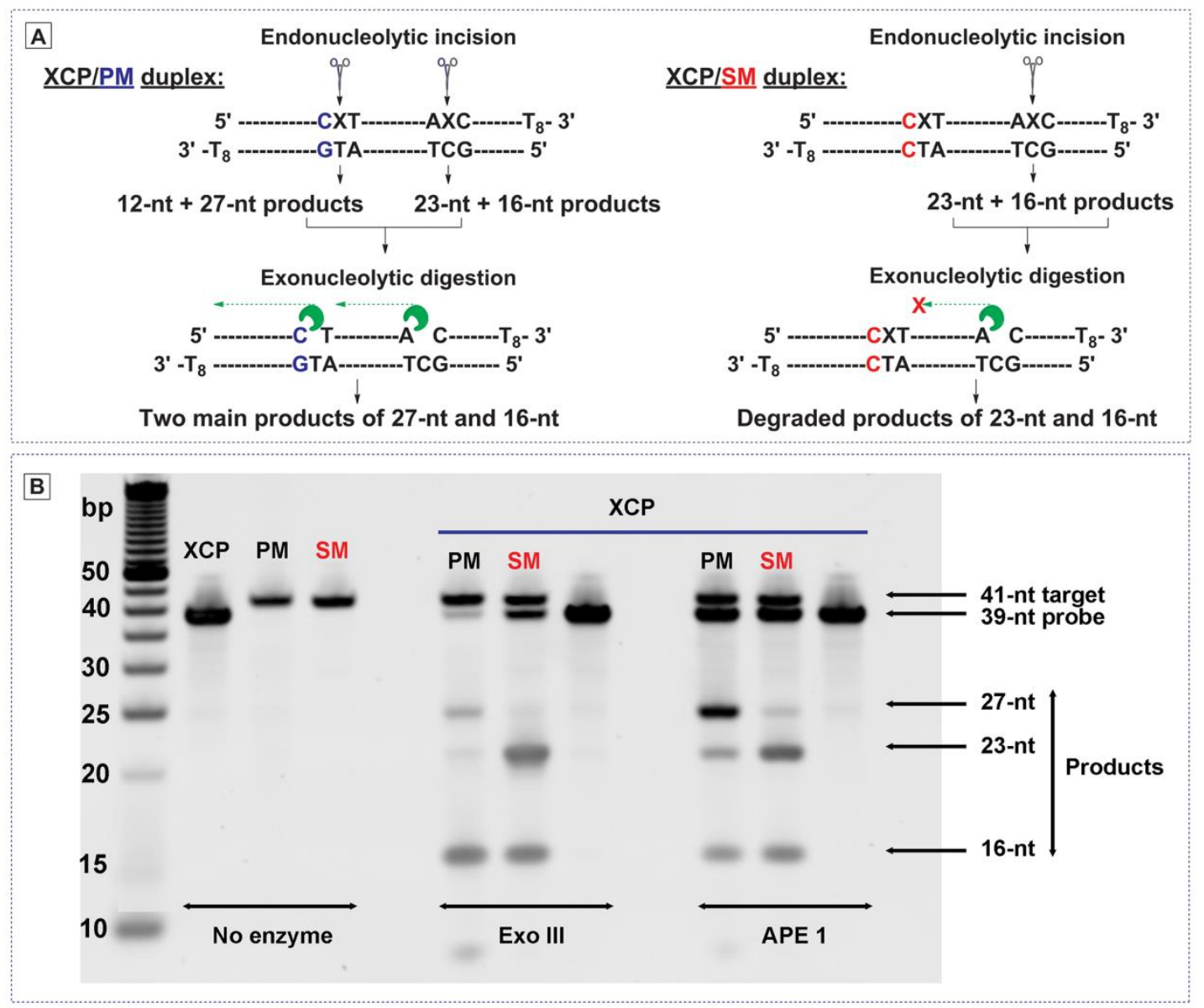

Figure 46. SNP discrimination achieved through the endonucleolytic incision of either Exo III or APE 1. (A) APE1 was unable to generate an amplified signal via target recycling due to the absence of exonuclease activity. (B) Gel image of unmodified CP (4) alone or with the poly $\left(\mathrm{T}_{8}\right)$ protected PM (5) or SM (6) target (10:1 probe: target ratio), either without enzyme, or incubated with 10 units of Exo III or $100 \mathrm{nM}$ APE 1. After heating at $80{ }^{\circ} \mathrm{C}$ for 10 minutes to stop the reaction, we characterized the samples via $12 \%$ PAGE.

The APE 1 can also efficiently cleave both the $5^{\prime}$ and $3^{\prime}$ abasic sites of the probe when hybridized with the PM target (Figure 46A). The enhanced cleavage at the matched 5' abasic site was clearly observed with the PM target, producing an increased amount of the 27-nt fragment (Figure 46B, PM with APE 1). This is because the 5' matched abasic site exhibits a higher melting temperature $\left(\mathrm{T}_{\mathrm{m}}=51.5^{\circ} \mathrm{C}\right)$ than the $3^{\prime}$ matched abasic site $\left(\mathrm{T}_{\mathrm{m}}=44.6{ }^{\circ} \mathrm{C}\right)$, which increased thermal stability and resulted in a higher concentration of formed duplexes and thus a higher endonuclease cleavage efficiency. ${ }^{275}$ Compared with Exo III, APE 1 failed to cleave the 
mismatched 5' abasic site in the CP/SM sample, producing only a minimal amount of 27-nt product, but yielded a large quantity of 23- and 16-nt products due to efficient cleavage at the matched 3' abasic site (Figure 46B, SM with APE 1). However, since APE 1 does not possess 3'to-5' exonucleolytic activity, each of these cleaved products appeared as a sharp, clear band in the gel. In contrast to the Exo III-treated PM sample, most of the 39-nt probe remains unprocessed in the APE 1-treated PM sample (Figure 46B). In the absence of exonucleolytic digestion, it is most likely that each target molecule only hybridizes with a single CP molecule, with no subsequent target recycling to amplify the reaction. Since all of the nicked duplexes have a $T_{m}$ well above room temperature, thermally induced dissociation of CP-target complexes is also unlikely.

As discussed above, both the apurinic endonucleolytic and 3' to 5' exonucleolytic activities of Exo III are critical to the assay. The Exo III-assisted digestion and target recycling in the PM sample resulted in complete removal of $\mathrm{CP}$ strands from the AuNP surface, which led to the saltinduced aggregation and yielded a visible color change. In contrast, although APE 1 can successfully discriminate the SNP by selective endonucleolytic cleavage at the matched abasic site, the subsequent target recycling failed because of the lack of exonuclease $3^{\prime}$ to $5^{\prime}$ activity. Thus, the remaining fragments and probes attached on the AuNPs could effectively protect AuNPs from salt-induced aggregation; thereby no color change was observed.

\subsubsection{Divalent cation effect}

Exo III is an enzyme with AP endonuclease and exonuclease activities that are highly dependent on divalent cations. ${ }^{272}$ To achieve optimal speed and accuracy of SNP detection, we assessed the impact of $\mathrm{Mg}^{2+}$ and $\mathrm{Ca}^{2+}$ concentrations on Exo III activity. We achieved this by measuring the single-base mismatch discrimination factor (DF) at different concentrations of calcium and magnesium ions. The DF is defined as the ratio of the net colorimetric signal gain at $650 \mathrm{~nm}$ (after subtracting background from probe only) obtained from AuNPs with the PM target relative to that obtained with the SM target; thus, a larger DF is indicative of better specificity. 
$\mathrm{Ca}^{2+}$ is a well-known analog of $\mathrm{Mg}^{2+}$ that contributes to the DNA binding specificity of Exo III for its endonuclease function. ${ }^{276}$ It has been known that Exo III presented only $7 \pm 2 \%$ of its endonucleolytic activity to the abasic site with a $\mathrm{C}-\mathrm{C}$ mismatch placed $5^{\prime}$ to the lesion in the presence of $\mathrm{Ca}^{2+}$. However, a $100 \%$ endonucleolytic activity was observed with its perfectlymatch C-G pair under the same condition. ${ }^{272}$
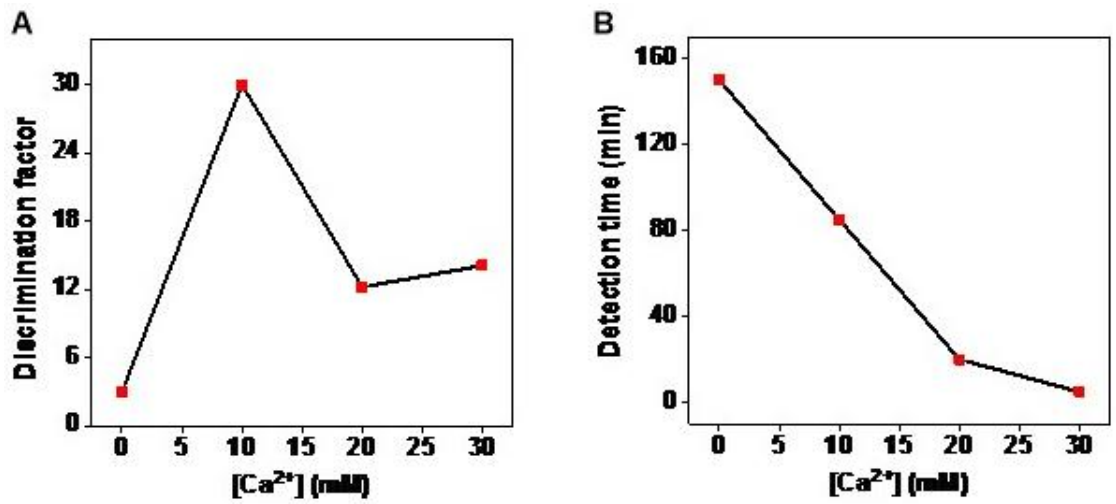

Figure 47. The influence of $\mathrm{Ca}^{2+}$ concentration on target discrimination (A) and detection time (B). Detection was performed in the buffer ( $\mathrm{pH}$ 7.9) composed of $20 \mathrm{mM}$ Tris-acetate, $50 \mathrm{mM}$ $\mathrm{KAc}, 25 \mathrm{mM} \mathrm{NaCl}$ and $3 \mathrm{mM} \mathrm{Mg}^{2+}$, with variable concentrations of $\mathrm{Ca}^{2+} .50 \mathrm{U}$ of Exonuclease III, $300 \mathrm{nM}$ CP-modified AuNPs and $20 \mathrm{nM}$ PM or SM were used in the reaction.

To maximize SNP discrimination, the concentration effect of $\mathrm{Ca}^{2+}$ on the colorimetric SNP detection was investigated. The concentrations of $\mathrm{Ca}^{2+}$ were tested in the range of 0 to $30 \mathrm{mM}$ in the presence of $3 \mathrm{mM} \mathrm{Mg}{ }^{2+}$ to produce a high-efficiency Exo III reaction. The experimental results showed very weak discrimination ability between PM and SM in the absence of $\mathrm{Ca}^{2+}$, since only modest discrimination $(\mathrm{DF}=2)$ was observed after 150 minutes (Figure 47$)$. When we increased the $\mathrm{Ca}^{2+}$ concentration to $10 \mathrm{mM}$, although a maximum DF of 30 was observed, the reaction took up to 85 minutes (Figure 47 ). As the $\mathrm{Ca}^{2+}$ concentration increased to $20 \mathrm{mM}$, the DF decreased to 12 , and the detection time was decreased to 15 minutes-5-fold faster than the reaction with the presence of $10 \mathrm{mM} \mathrm{Ca}^{2+}$, presumably due to the high $\mathrm{Ca}^{2+}$-induced aggregation of AuNPs (Figure 47). While, if the concentration of $\mathrm{Ca}^{2+}$ increased to $30 \mathrm{mM}$, the DF was saturated, and AuNPs aggregation was observed in 5 minutes. Although the presence of $\mathrm{Ca}^{2+}$ 
enhance formation of the protein-metal-DNA complex, ${ }^{276}$ these ions can also inhibit the enzyme's endonucleolytic activity. It is clear that $\mathrm{Ca}^{2+}$ mainly affects the AP-endonucleolytic activity of Exo III to specifically cleave the mismatched abasic site. Thus, $20 \mathrm{mM} \mathrm{Ca}^{2+}$ was used to optimize a faster SNP detection.
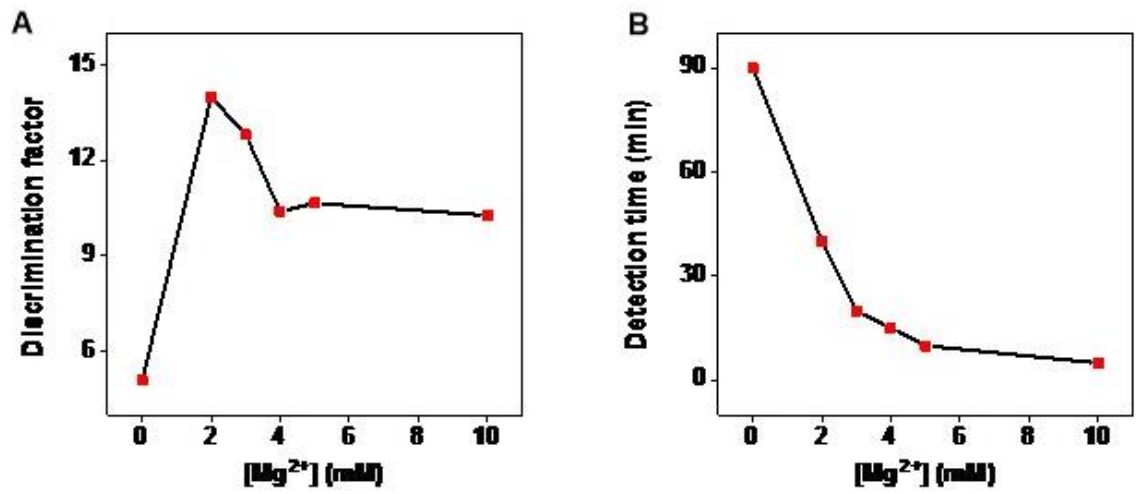

Figure 48. The influence of $\mathrm{Mg}^{2+}$ concentration on target discrimination (A) and detection time (B). The reaction buffer ( $\mathrm{pH}$ 7.9) contained $20 \mathrm{mM}$ Tris-acetate, $50 \mathrm{mM} \mathrm{KAc}, 25 \mathrm{mM} \mathrm{NaCl}$ and $20 \mathrm{mM} \mathrm{Ca}^{2+}$, with variable concentrations of $\mathrm{Mg}^{2+}$. $50 \mathrm{U}$ of Exo III, $300 \mathrm{nM} \mathrm{CP-modified} \mathrm{AuNPs}$ and $20 \mathrm{nM}$ PM or SM were used in the reaction.

It is known that $\mathrm{Mg}^{2+}$ strongly stimulates the Exo III activity due to its suitable ionic radius and electronegativity. ${ }^{277}$ Thus, the concentration of $\mathrm{Mg}^{2+}$ was also explored from 0 to $10 \mathrm{mM}$ with the fixed concentration $\mathrm{Ca}^{2+}(20 \mathrm{mM})$. It was found that the activity of Exo III increased as the $\mathrm{Mg}^{2+}$ concentration increased, and a maximum DF of 14 was achieved in the presence of $2 \mathrm{mM}$ $\mathrm{Mg}^{2+}$ after 40 minutes (Figure 48). However, along with the further increase of the $\mathrm{Mg}^{2+}$ concentration, the reaction rate accelerated, but the DF decreased due to nonspecific digestion of probe DNA by Exo III. It was observed that the DF decreased to a plateau of 10 when $\mathrm{Mg}^{2+}$ concentration is higher than $4 \mathrm{mM}$ (Figure 48). In order to achieve fast reaction speed and good specificity, reaction buffer containing $3 \mathrm{mM}$ of $\mathrm{Mg}^{2+}$ and $20 \mathrm{mM}$ of $\mathrm{Ca}^{2+}$ was employed for all reactions. This combination ensures that Exo III distinguishes the perfectly-matched and singlebase mismatched abasic sites and performs rapid exonucleolytic digestion of the resulting fragments, yielding good specificity within 15 minutes. 


\subsubsection{Kinetics of Exo III digestion}

The AuNPs can only form aggregates when the majority of the immobilized CP probes are sheared from the surface. It is believed that exonuclease digestion-mediated AuNPs aggregation is the rate-limiting step because the hybridization between the AuNPs-attached CP probe and target is quick in homogenous solution. ${ }^{278}$ Time course reactions were performed between CPmodified AuNPs with PM or SM, and the rate constants of the enzymatic reaction were estimated by fitting the time course data to the Michaelis-Menten equation (Figure 49A). Different concentrations of the PM were tested, and the absorbance difference $(\Delta \mathrm{A})$ at $524 \mathrm{~nm}$ between AuNPs-attached probe was monitored without and with PM. Michaelis-Menten constant $\left(K_{\mathrm{m}}\right)$ of $426 \pm 20 \mathrm{nM}$ and an apparent turn-over number for target DNA $\left(k_{\text {cat }}\right)$ of $1.26 \mathrm{~min}^{-1}$ were obtained, and a reaction efficiency $\left(k_{\mathrm{cat}} / K_{\mathrm{m}}\right)$ of Exo III was $0.0030 \mathrm{~min}^{-1} \mathrm{nM}^{-1}$ (Figure 49B). In contrast, reaction rate constant for the SM substrate was impossible to obtain due to a much lower Exo III activity. The $K_{\mathrm{m}}$ value for our AuNPs-attached CP system is $\sim 3$ times lower than that of Exo III observed in solution, ${ }^{272}$ suggesting that the $k_{\text {cat }}$ in our SNP assay incorporates the rate constraints for incision and digestion of Exo III and the aggregation of AuNPs.
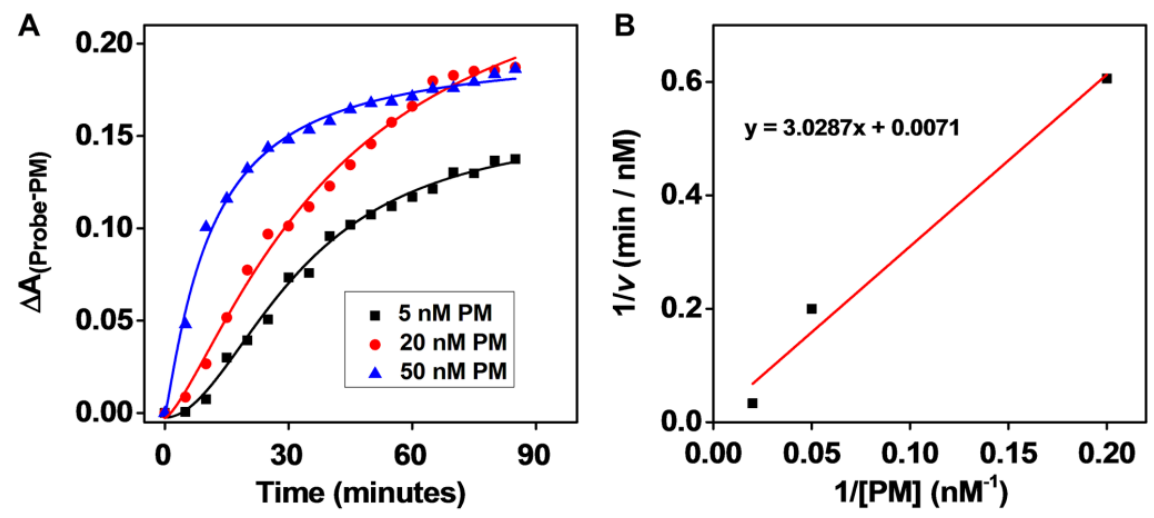

Figure 49. Determination of Exo III kinetics in the AuNP-attached CP system. (A) Time course of our SNP sensor at different PM target concentrations. The absorbance difference $(\Delta \mathrm{A})$ between AuNPs-attached probe alone and with PM was recorded at $524 \mathrm{~nm}$. (B) The rate constant of the Exo III reaction calculated by fitting time-course data to the Michaelis-Menten equation. 
To test the dynamic range of our SNP assay, different concentrations (from $10 \mathrm{nM}$ to 100 $\mathrm{nM})$ of the PM or SM target were added into the CP-modified AuNPs and the mixture was incubated for a 10-minute reaction with 50U Exo III. Under these reaction conditions, a detection limit for PM target as low as $10 \mathrm{nM}$ was obtained at room temperature after 5 minutes, which the color change was strong enough to be observed by the naked eye (Figure 50). However, there was almost no color change observed for the SM target within 2 hours.

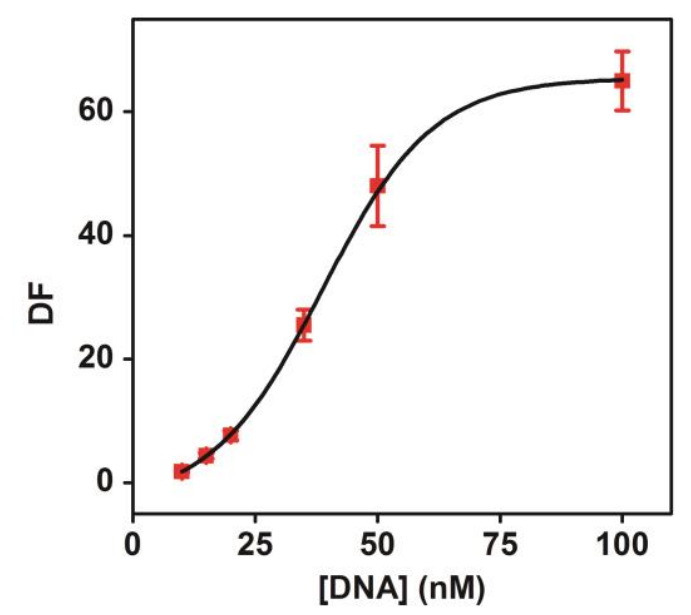

Figure 50. The dynamic range of the AuNP-based, Exo III-amplified sensor (10 nM - $100 \mathrm{nM})$ after a 10-minute reaction. Detection was performed in $20 \mathrm{mM}$ Tris-acetate buffer ( $\mathrm{pH} 7.9$ ) composed of $50 \mathrm{mM} \mathrm{KAc}, 25 \mathrm{mM} \mathrm{NaCl}, 3 \mathrm{mM} \mathrm{Mg}^{2+}$ and $20 \mathrm{mM} \mathrm{Ca}^{2+}$. $50 \mathrm{U}$ of Exo III, $300 \mathrm{nM}$ CP-modified AuNPs and variable concentrations of PM or SM were used in the reaction. The error bars represent the standard deviation of three measurements conducted at each target concentration.

We further performed the reaction at $4{ }^{\circ} \mathrm{C}$ and found that the detection limit could reach as low as $2 \mathrm{nM}(\mathrm{S} / \mathrm{N}>3)$ and the color change was still good enough for naked eye observation after 4 hours (Figure 51). The sensitivity of our SNP assay was dramatically improved at a lower temperature; however, there is a trade-off between reaction time and limit of detection. Although the time is slightly longer than usual, it significantly simplified the detection procedure compared to other reported SNP assays and maintained good specificity and sensitivity. We attributed the high sensitivity to the Exo III, which triggered the signal amplification process. This color change 
was easily observed by naked eye with as little as $2 \mathrm{nM}$ of the target, which is 100 times lower compared to the concentration required for unmodified AuNPs under the documented conditions. $^{156}$

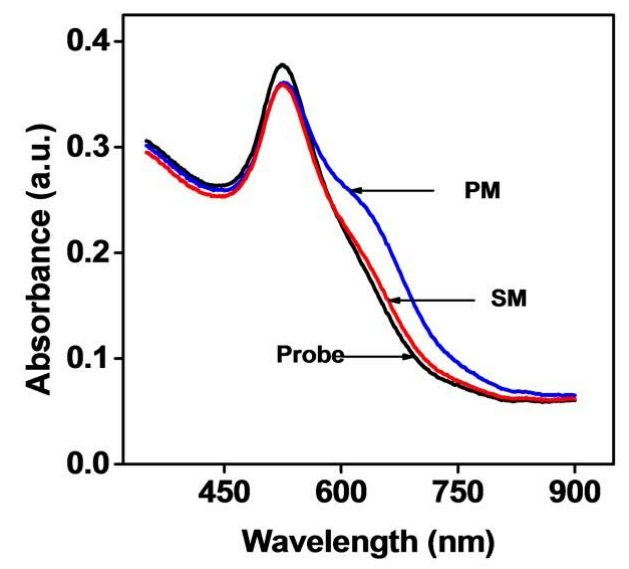

Figure 51. A detection limit of $2 \mathrm{nM}(\mathrm{S} / \mathrm{N}>3)$ achieved by performing the reaction at $4{ }^{\circ} \mathrm{C}$ for 4 hours. In the presence of $50 \mathrm{U}$ Exo III, UV-vis spectra of CP-modified AuNPs alone (probe only), or with $2 \mathrm{nM}$ SM or PM, the color change was great enough for naked eye observation.

\subsubsection{SNP detection}

To demonstrate the generality of this sensor platform, the discrimination ability against different mismatched bases [C-C, C-T and C-A] located at the $5^{\prime}$ the abasic site with the PM target was explored. The solution containing PM target generated a blue color after the Exo III digestion in 15 minutes, whereas any single-base mismatch $(\mathrm{C}, \mathrm{T}$ or $\mathrm{A}) \mathrm{DNA}$ target remained red after 2 hours (Figure 52A), indicating the good discrimination ability of the SNP detection. The highest level of discrimination $(\mathrm{DF}=12)$ was observed in the duplex containing a $\mathrm{C}-\mathrm{C}$ mismatch, whereas the lowest level $(\mathrm{DF}=8.5)$ was observed with a $\mathrm{C}-\mathrm{T}$ mismatch. The difference is consistent with the assumption that mismatch thermodynamic depends not only on the mismatched base pair but also the identity of its near neighbors. ${ }^{279}$ However, the experimental results are in contrast to the literature report that the endonucleolytic activity of Exo III is only inhibited by a C-C mismatch positioned $5^{\prime}$ to an abasic site. ${ }^{272}$ When we switched single-base 
mismatches [T-T and T-G] from the $5^{\prime}$ to the $3^{\prime}$ side of the $5^{\prime}$ abasic site, all of the solution changed to blue color, regardless of the PM or SM. The discrimination of single-base mismatches was not observed anymore (Figure 52B), confirming that Exo III-mediated SNP discrimination is based on its $5^{\prime}$ endonucleolytic activity. ${ }^{280}$
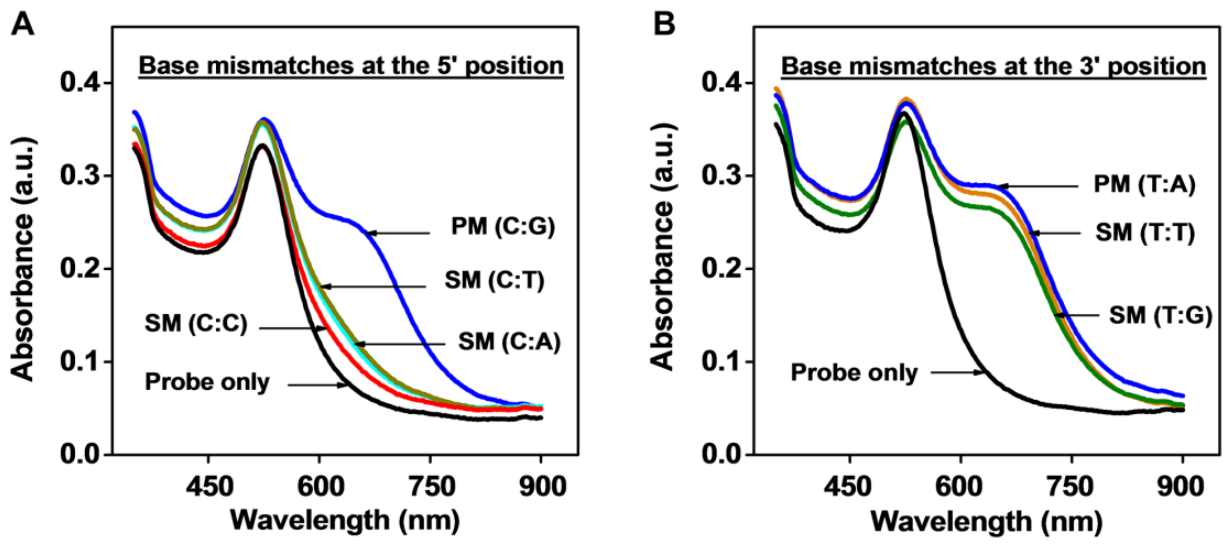

Figure 52. The generality of mismatch detection of the AuNP-based, Exo III-amplified sensor. A) Our sensor can effectively discriminate SM from PM regardless of which mismatched base is located at the 5' abasic site. B) Our sensor could not discriminate mismatches positioned 3' to the 5' abasic site, due to the 5' endonucleolytic activity of Exo III. Data show UV-vis spectra of CPmodified AuNPs probe alone or with $20 \mathrm{nM}$ PM or SM sequences after a 15-minute incubation with Exo III at room temperature.

\subsection{Conclusion}

A remarkably simple platform that enables sensitive, naked-eye detection of SNPs at room temperature within 15 minutes has been developed. This one-pot assay was performed in a single tube with one set of DNA probe-modified AuNPs, a single enzyme, and the target DNA. In the system, capture probes immobilized on the AuNP were first hybridized with the target and were then selectively cleaved into different fragments at abasic sites. In the presence of a perfectlymatched target, target-probe duplexes were first endonucleolytic cleaved by Exo III at both abasic sites on the probe to form nicked duplexes, which were rapidly degraded via the exonucleolytic action of Exo III by the release of the target for the recycling, leading to improved sensitivity. 
The released target was used to hybridize/digest another probe to start another cycle. Sequenceindependent Exo III was used to "recycle" target molecules for multiple times, thus amplified the signal from the PM target, and ultimately stripped all probes from the AuNPs, resulting in saltinduced AuNPs aggregation along with a visible red-to-blue color change. In contrast, the mismatched targets inhibited the Exo III's endonucleolytic activity, stalling exonucleolytic digestion, so the AuNPs solution remained red in color.

In our assay, the AuNPs serve not only as an active substrate for DNA modification but also as a sensitive signal reporter due to their extremely large molar extinction coefficient and excellent sensitivity to SNP associated aggregation, which can directly report the SNP identification by a red-to-blue color change without the need of any additional instrument. However, the current detection limit $(2 \mathrm{nM})$ is still not sufficient to omit a target amplification step. To obtain even lower clinical level sensitivity $(\sim \mathrm{fM})$, another amplification strategy needs to be employed to achieve real-time SNP detection. Since the AuNP aggregation is induced by Exo III-mediated DNA shearing from the particle surface, the developed system could also be rendered in complex biological samples by using nuclease-resistant phosphorothiolated probes.

Most importantly, the SNP detection system can readily discriminate SNP-containing targets regardless of which the mismatched base is positioned at the 5' abasic site, which enables direct SNP detection without the design requirement of complicated probes. The entire analysis process is simple, fast and cheap, requiring only a one-step mixture reaction, without any complex labeling and pretreated process. Therefore, this method should have great prospects for point-ofcare analysis of practical biosamples. Additionally, our one-step, one-pot colorimetrical SNP multi-target, parallel SNP analysis over molecule beacons ${ }^{281}$ and TaqMan probes, ${ }^{282}$ which often require delicate probe design and precise temperature control. It is worth to note that the colorimetric detection platform can be easily transferred into a multiplexed, paper-based device to permit rapid, cheap and immediate screening of SNPs. Given that a short, abasic site inserted 
oligonucleotide could be introduced to hybridize the target-binding portion of any aptamer, ${ }^{283}$ we believe that our platform can be highly generalized for the detection of a variety of non-DNA targets, including proteins, small molecules, metal ions and even whole cells. 
CHAPTER 5: Effect of DNA Surface coverage on the performance of aptamer-based sensor platform for small molecule detection

\subsection{Introduction}

DNA functionalized AuNP conjugates served as unique probes for recognizing specific sequences in DNA segments and as building blocks for assembling novel structures and materials. It has been well proved that the surface coverage of the DNA plays an important role in the DNA biosensor's stability, sensitivity, and selectivity. ${ }^{164,166,167}$ The surface coverage of the immobilized ssDNA determines the DNA orientation at the interface and thus the efficiency of the hybridization process. The high surface density of the probe leads to increasing steric and electrostatic repulsion between the probe and target, resulting in low hybridization efficiency and subsequently a longer reaction time. At low surface density, steric hindrance can be significantly reduced, thus enhancing hybridization efficiency for target binding. ${ }^{165}$ However, the DNA strands are likely lying on the gold substrate surface due to non-specific adsorption. Therefore, an optimization of probe density is necessary to provide sufficient hybridization efficiency and faster reaction time. Maximum signals are normally achieved when the amount of target DNA hybridizing with immobilized probe is maximized.

The loading of DNA oligonucleotides on the Au substrate can usually be controlled during the modification step by varying ionic strength, base composition, DNA concentration, or coadsorption with thiolated molecules including MCH or DTT. ${ }^{167,169,170,284}$ Those simple single-step assembly procedures allow control of the surface density of DNA on the gold surface. However, multiple modifications are required to obtain different surface coverages. In addition, careful experimental design is required; for example, high salt leads to irreversible aggregation of AuNPs during the modification. The surface coverage can only be tuned within a limited range; it is going to be troublesome when precise reproducibility of the DNA density is required. Two-step methods also have been reported to precise control over surface coverage on the gold substrate. 
The DNA surface density can be decreased by the addition of shorter thiolated molecules via ligand-exchange reaction. The mixed monolayers were formed on the gold surface when exposed to DNA solution first, followed by exposure to $\mathrm{MCH}$ solution. A primary advantage of using this two-step process is that not only nonspecifically adsorbed DNA is largely removed from the surface, but also remove some of the chemically attached DNA from the surface by controlling the exposure time to the diluent solution or varying the diluent concentration. Thus, almost all of the surface-bound probes are available for specific hybridization with DNA targets.

The objective of this chapter is to evaluate the DNA surface coverage accurately on the AuNPs surface, develop a simple method to control the DNA surface coverage, test the surface coverage effect on the DNA and small molecule detection. First, we evaluated the commercially available OliGreen dye on the characterization of the DNA surface coverage. Second, we developed a simple and rapid assay to regulate the surface coverage after one-pot synthesis of DNA-modified AuNP by backfilling the oligonucleotides immobilized on the AuNP surface with two small thiolated molecules - MCH and DTT - and then tested surface coverage effect on the DNA detection using the enzyme-assisted target recycling (EATR) method. ${ }^{70}$ In order to demonstrate how the DNA surface coverage also affects small molecule detection, the cocaine aptamer was chosen as a model and we developed a colorimetric detection for cocaine based on Exo III inhibition on the target-aptamer complex.

\subsection{Materials and methods}

Chemicals and materials All DNA strands were purchased from Integrated DNA Technologies (USA) and purified by HPLC. Tris (2-carboxyethyl) phosphine hydrochloride (TCEP), 6-mercapto-1-hexanol (MCH), Dithiothreitol (DTT), cocaine hydrochloride, NaCl, $\mathrm{MgCl}_{2}$, Trizma preset crystal, Tris-EDTA buffer were purchased from Sigma-Aldrich. Exonuclease III was purchased from New England Biolabs (NEB). Water was purified by an Ultrapure Milli-Q water system and used to prepare all solutions. SYBR® Gold Nucleic Acid Gel 
Stain $\left(10,000 \mathrm{X}\right.$ Concentrate in DMSO) and Quant-iT ${ }^{\mathrm{TM}}$ OliGreen ${ }^{\circledR}$ ssDNA Reagent were purchased from Thermo Fisher Scientific. Amicon Ultra-4 Centrifugal Filter columns (100KD) were purchased from Millipore.

Modification of DNA on AuNPs All glasswares were incubated in aqua regia solution for overnight and rinsed thoroughly with tap water followed by deionized water. The AuNPs (13nm) were prepared using the method described in chapter 4. The DNA-modified AuNPs were prepared by gold-sulfur covalent chemistry. ${ }^{5,72}$ The thiolated oligonucleotide ( 7.95 nmoles) was first mixed with freshly prepared TCEP (601.8 nmoles) for 2 hours at room temperature to reduce the disulfide bond. The reduced DNAs were then added into $3 \mathrm{~mL}$ freshly synthesized AuNP solution $(9.6 \mathrm{nM})$. The mixture was incubated at room temperature for 12 hours, followed by the slow addition of $4 \mathrm{M} \mathrm{NaCl}(50 \mu \mathrm{L})$. The salt-aged mixture was incubated at room temperature for another 12 hours and aged with another $100 \mu \mathrm{L}$ of $4 \mathrm{M} \mathrm{NaCl}$. The resulting solution was incubated at room temperature for another 6 hours before addition of $100 \mu \mathrm{L}$ of $4 \mathrm{M} \mathrm{NaCl}$. After incubating the mixture at room temperature for another 12 hours, the solution was centrifuged at $1500 \mathrm{rcf}$ for 10 minutes at $4{ }^{\circ} \mathrm{C}$ using Amicon Ultra-4 Centrifugal Filter column (Millipore 100KD) to remove the free unconjugated DNA and an excess amount of $\mathrm{NaCl}$. The resultant AuNP pellet was washed with 10mM Trizma buffer (pH 7.4) 6 times. Finally, the DNA-modified AuNPs were suspended in the same buffer to obtain a particle concentration about $100 \mathrm{nM}$.

\section{Characterization of DTT-displaced, FAM-labeled DNA in solution and on gel The} surface coverage of the FAM-labeled DNA modified AuNP was first characterized using the DTT-displacement assay. ${ }^{169}$ To do so, $5 \mu \mathrm{L}$ of DNA-modified AuNPs solution was diluted with $45 \mu \mathrm{L}$ of $10 \mathrm{mM}$ Tris buffer (pH 7.4), and then mixed with $50 \mu \mathrm{L}$ of $1.0 \mathrm{M}$ DTT (prepared in 10 $\mathrm{mM}$ Tris, $\mathrm{pH}$ 7.4) thoroughly. After incubation at room temperature for 16 hours, the solution was centrifuged at 21,130 ref for 30 minutes to remove the AuNP precipitate, and $80 \mu \mathrm{L}$ of supernatant was transferred into a 96-well microplate to record the fluorescence spectra from 505 
$\mathrm{nm}$ to $850 \mathrm{~nm}\left(\lambda_{\mathrm{ex}}=495 \mathrm{~nm}\right)$. To establish the standard calibration curve, $5 \mu \mathrm{L}$ of $10 \mathrm{mM}$ Tris buffer containing known concentrations of unconjugated SH-probe was diluted with $45 \mu \mathrm{L}$ of 10 $\mathrm{mM}$ Tris buffer and mixed with $50 \mu \mathrm{L}$ of $1.0 \mathrm{M}$ DTT (prepared in $10 \mathrm{mM}$ Tris buffer, $\mathrm{pH}$ 7.4). The samples used for the calibration underwent a 16-hour incubation and the same centrifugation process as the DNA-modified AuNP samples.

The DTT-displaced, FAM-labeled DNA samples were also characterized by denaturing PAGE. A $5 \mu \mathrm{L}$ of DTT-displaced DNA samples or standard unconjugated DNA solutions with different concentrations were mixed with $10 \mu \mathrm{L}$ formamide loading buffer $(71.25 \%$ formamide, $10 \%$ glycerol, $0.125 \%$ SDS, $25 \mathrm{mM}$ EDTA, and $0.15 \%(\mathrm{w} / \mathrm{v})$ bromophenol blue and xylene cyanol). The prepared samples and standards DNA solution were loaded into each well of a $15 \%$ denaturing PAGE gel. Separation was carried out at $20 \mathrm{~V} / \mathrm{cm}$ for 1 hour in $0.5 \times$ TBE $(44.5 \mathrm{mM}$ Tris base, $44.5 \mathrm{mM}$ boric acid, $1 \mathrm{mM}$ EDTA) running buffer. After the gel electrophoresis assay was complete, the DNA was imaged directly using a ChemiDoc MP imaging system (Bio-Rad) or stained with $0.5 \times$ SYBR Gold for 25 minutes before the gel imaging. Comparing band intensity using a known concentration of DNA sample allowed us to generate a calibration curve and calculate DNA concentration of the DTT-displaced samples. Since FAM emits a fluorescence signal under UV light, we directly imaged the gel after DNA separation. We plotted the band intensity versus the standard concentrations of FAM-labeled, unconjugated DNA to form a calibration curve. The amount of DNA modified on AuNP was then calculated using this generated calibration curve. The resultant gel was also stained with $0.5 \times$ SYBR Gold. After the staining, the band intensity was re-analyzed and re-plotted with the standard concentrations of FAM-labeled, unconjugated DNA.

\section{Characterization of DTT-displaced unlabeled DNA stained with either SYBR Gold on}

gel or OliGreen in solution The unlabeled, DNA-modified AuNP were treated with an equal volume of 1.0 M DTT to release the DNA into the solution. After the separation of AuNPs from 
the DNA supernatant, the DTT-displaced, unlabeled DNA solution was first characterized by denaturing PAGE. A $5 \mu \mathrm{L}$ of standard unlabeled and unconjugated DNA solution at different concentrations or the DTT-displaced DNA samples were mixed with $10 \mu \mathrm{L}$ formamide loading buffer. The prepared standards and sample were then loaded into each well of the $15 \%$ denature PAGE gel. Separation was carried out at $20 \mathrm{~V} / \mathrm{cm}$ for 1 hour in $0.5 \times$ TBE running buffer. After the separation was complete, the gel was stained with $0.5 \times$ SYBR Gold for 25 minutes and imaged using a ChemiDoc MP imaging system (Bio-Rad). The calibration curve generated by the band intensity of known concentrations of standards allowed us to calculate the DNA surface coverage of unlabeled DNA on AuNPs.

The amount of unlabeled DNA on AuNPs was also determined using a commercially available single-stranded oligonucleotide quantification kit (OliGreen; Invitrogen). The stock concentration of OliGreen dye is $200 \times$. To perform the measurements, $20 \mu \mathrm{L}$ of the DTTdisplaced DNA supernatant was diluted with $80 \mu \mathrm{L}$ of $1 \times$ TE buffer $(10 \mathrm{mM}$ Tris-HCl, $1 \mathrm{mM}$ EDTA, pH 8.0), and then mixed with $100 \mu \mathrm{L}$ of $1 \times$ OliGreen prepared with $1 \times$ TE buffer for 5 minutes in the dark. The mixture was then loaded into a 96-well plate. The OliGreen dye specifically binds with ssDNA, emitting a strong fluorescence at $525 \mathrm{~nm}$. Fluorescence intensities were recorded at $525 \mathrm{~nm}$ using a Tecan Infinite M1000 PRO with an excitation wavelength of $500 \mathrm{~nm}$. To calculate DNA surface coverage, we established a standard curve by mixing different concentrations of unlabeled, unconjugated DNA with $1 \times$ OliGreen prepared with $1 \times$ TE buffer.

Effect of Exo III activity on the MCH (or DTT)-regulated DNA surface coverage MCH (or DTT) displacement was carried out to reduce the DNA surface coverage on AuNPs via ligand exchange reaction. The displacement was performed by mixing $50 \mu \mathrm{L}$ DNA-modified AuNPs with $20 \mu \mathrm{L}$ of different concentrations of $\mathrm{MCH}$ (or DTT) in $10 \mathrm{mM}$ Tris buffer ( $\mathrm{pH}$ 7.4) for 30 minutes. The excess MCH (or DTT) and displaced DNA were then removed from the solution by centrifugation. To test the effect of $\mathrm{MCH}$ (or DTT)-regulated surface coverage on Exo III 
activity, the modified DNAs were dispersed in $10 \mathrm{mM}$ Tris buffer solution containing $20 \mathrm{mM}$ $\mathrm{NaCl}$ and $6 \mathrm{mM} \mathrm{MgCl}$. The time course of Exo III digestion was monitored in the presence and absence of $100 \mathrm{nM}$ DNA target upon addition of $1 \mathrm{U}$ of Exo III, with the absorbance ratio at 650 to $520 \mathrm{~nm}\left(\mathrm{~A}_{650} / \mathrm{A}_{520}\right)$ serving as an indicator of AuNP aggregation.

To characterize the resulting surface coverage on AuNPs after MCH (or DTT) treatment, the concentration of AuNPs and the concentration of fluorescent DNA in each sample were measured according to a previously reported method. ${ }^{169}$ Specifically, the AuNP pellet was re-suspended in $10 \mathrm{mM}$ Tris buffer and mixed with an equal volume of 1.0 M DTT. The mixture was incubated overnight at room temperature to displace all DNA strands from the AuNP surface. The supernatant was collected after centrifugation, and the fluorescence was measured after incubation with $1 \times$ OliGreen dye for 5 minutes in the dark. Standard DNA samples were prepared under the same salt and buffer conditions and mixed with $1 \times$ OliGreen dye to generate a calibration curve. Fluorescence intensities were recorded at $525 \mathrm{~nm}$ with an excitation wavelength of $500 \mathrm{~nm}$. The concentration of AuNPs was calculated by using Beer's Law and $\varepsilon=$ $2.7 \times 10^{8} \cdot \mathrm{mol}^{-1} \cdot \mathrm{cm}^{-1}$.

Characterize Exo III inhibition effect on the cocaine split aptamer on gel To test Exo III inhibition on the target-aptamer complex, a cocaine split aptamer (38-GC) containing a singlebinding domain or a newly engineered cocaine split aptamer - cooperative binding split aptamer (5325-AT or 5325-XT) - containing dual binding domains was incubated with 10 U Exo III in Tris buffer ( $\mathrm{pH} 7.4$ ) including $125 \mathrm{mM} \mathrm{NaCl}$ and $0.1 \mathrm{mM} \mathrm{MgCl}_{2}$ in the absence or presence of $250 \mu \mathrm{M}$ cocaine. The reaction was stopped at each selected reaction time by mixing the sample with formamide loading buffer. A $3 \mu \mathrm{L}$ of each mixture was loaded into the well and the digested products from different reaction times were characterized by $15 \%$ denaturing PAGE gel. Separation was carried out at $20 \mathrm{~V} / \mathrm{cm}$ for 3 hours in $0.5 \times$ TBE running buffer. The gel was stained with $100 \mathrm{~mL}$ of $0.5 \times$ SYBR Gold solution for 25 minutes and imaged using a ChemiDoc 
MP imaging system (Bio-Rad). Both 38-GC split aptamer and CBSA-5325 were tested under the same buffer condition, but challenged with different concentrations of Exo III.

\section{AuNP-based colorimetric detection of cocaine based on Exo III inhibition on target-}

aptamer complex Based on Exo III inhibition on the cocaine-bound CBSA, we have used CBSA-5325-XT to develop an AuNP-reported colorimetric detection of cocaine. We modified the CBSA short fragment (SH-5325-5T-SF) onto AuNP via thiol-gold chemistry as described above. To test the effect of DNA surface coverage on cocaine detection, the SF-modified AuNPs were treated with different concentration of DTT $(0-600 \mu \mathrm{M})$ or MCH $(0-1200 \mu \mathrm{M})$ to reduce the DNA surface coverage. The DTT (or MCH) treated DNA-modified AuNPs were then redispersed in $10 \mathrm{mM}$ Tris buffer ( $\mathrm{pH}$ 7.4) solution containing $125 \mathrm{mM} \mathrm{NaCl}$ and $0.1 \mathrm{mM} \mathrm{MgCl}$, and incubated with $1 \mu \mathrm{M}$ long fragment (named 5A-LF), $250 \mu \mathrm{M}$ cocaine or DI for 1 hour at room temperature. Upon addition of $10 \mathrm{U}$ of Exo III, the absorbance change of the samples were recorded. To determine the effect of thermodynamic stability of cocaine-aptamer complexes on Exo III digestion, CBSA long fragments modified with different poly A linker on the 3 ' end (3ALF, 4A-LF, 5A-LF and 6A-LF) were tested (Sequences list in Table 4). The DTT-treated DNAmodified AuNPs were redispersed in $10 \mathrm{mM}$ Tris buffer solution containing $125 \mathrm{mM} \mathrm{NaCl}$ and $0.1 \mathrm{mM} \mathrm{MgCl} \mathrm{Mn}_{2}$ and $1 \mu \mathrm{M}$ different long fragment. $10 \mathrm{U}$ of Exo III was then added into the sample containing $250 \mu \mathrm{M}$ cocaine or DI and the time course was monitored. To test the effect of CBSALF concentration on the sensor performance, different concentrations of CBSA 5A-LF $(0.1 \mu \mathrm{M}$ $8 \mu \mathrm{M})$ were added into the DTT-treated, DNA-modified AuNPs and the time course was recorded. The effect of cations including $\mathrm{Na}^{+}$and $\mathrm{Mg}^{2+}$ were also tested in the similar fashion. 


\subsection{Results and discussions}

\subsubsection{Evaluation of OliGreen fluorescence assay for the characterization of DNA surface coverage on AuNPs}

We have validated the method that characterized the surface coverage of unlabeled DNA on the AuNPs using OliGreen fluorescence dye. We first used a "gold standard" DTT-displacement method to characterize the surface coverage of three different FAM-labeled DNA sequences on AuNPs by measuring the fluorescence intensity of the displaced DNA strands in solution. We then compared the identified surface coverage with the results obtained with a common lab technique - gel electrophoresis. We found that the DNA surface coverage quantified with both methods were similar. Based on the fact that DNA surface coverage on AuNPs can be accurately measured by gel electrophoresis, the DNA surface coverage of another two non-FAM-labeled DNA sequences were further characterized with gel electrophoresis and OliGreen-based fluorescence measurement in solution. The results obtained from both methods were consistent,

proving that the OliGreen-based method enabled accurate measurement of surface coverage of unlabeled DNA on AuNPs.

5.3.1.1 Characterization of DTT-displaced, FAM-labeled DNA in solution and on the gel

Table 2. Three FAM-labeled thiolated DNA sequences employed in this work (from 5' to $\left.3^{\prime}\right)$.

\section{NoX-FAM:}

HS-TTT TTT ACC ACA TCA TCC ATAT AAC TGA AAG CCA AAC AG-FAM

\section{X-FAM:}

HS-TTT TTT ACC ACA TCA TCC /X/TAT AAC TGA AAG CCA AAC AGT TTT TTT T -FAM

2X-FAM:

TTT TTT ACC ACA TCA TCC /X/TAT AAC TGA AA/X/ C CAA ACA GTT TTT TTT FAM

${ }^{*} / \mathrm{X} /$ denotes the abasic site (a propyl residue, Spacer- $\mathrm{C}_{3}$ ), $1 \mathrm{X}=$ one abasic site and $2 \mathrm{X}=$ two abasic sites). $\quad$ */FAM/ denotes fluorescein. 
We first modified three different FAM-modified DNA strands (Table 2, NoX-FAM; 1XFAM and 2X-FAM) on AuNPs via thiol-gold chemistry. To accurately determine DNA surface coverage on AuNPs, the concentrations of AuNPs and DNA in each sample were measured. The UV-Vis measurement of AuNP solution was performed in the range of 300 to $800 \mathrm{~nm}$ and the concentration of AuNPs was calculated using Beer's Law with maximum wavelength located at $520 \mathrm{~nm}$. Fluorophore-labeled DNA is strongly quenched when bound to AuNPs (Figure 53, left), but the fluorescence of the labeled DNA is recovered once it is detached from the surface (Figure 53, right). Thus, DTT assay, a gold standard method to detach DNA from the particle, was used to obtain the amount of DNA immobilized on the AuNPs.

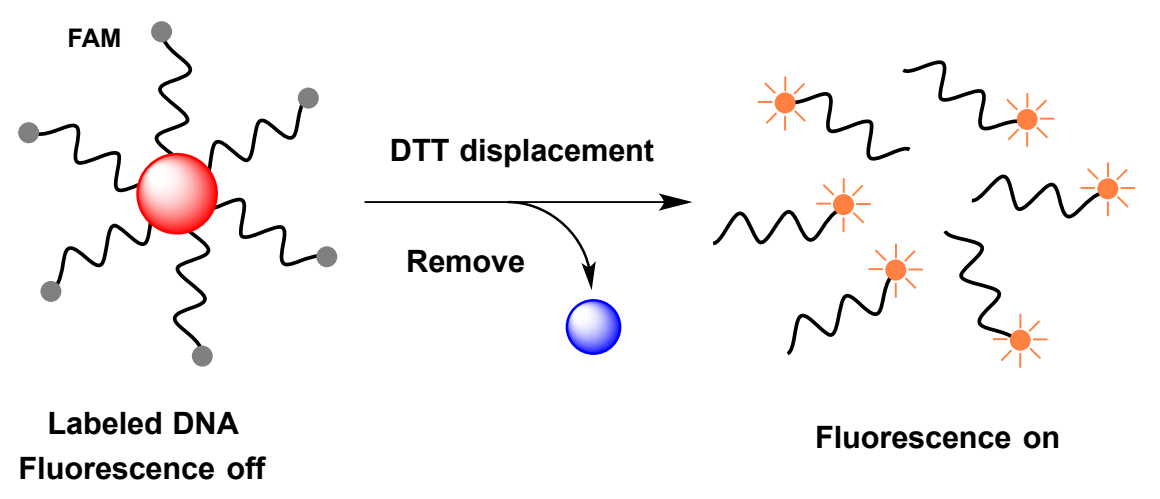

Figure 53. Scheme of DTT displacement of AuNP-conjugated fluorescein-labeled DNA strands.

DTT, a highly water-soluble solid agent that can effectively bind to gold surface via two thiol groups - was used to directly displace the immobilized DNA from AuNP surfaces via ligandexchange reaction. Specifically, the fluorescence of AuNP-conjugated 6-FAM labeled DNA was efficiently quenched as a result of fluorescence resonance energy transfer (FRET) to AuNP. After adding equal volumes of 1.0 M DTT into the FAM-labeled DNA-functionalized AuNPs for an overnight incubation, the FAM-DNA was completely removed from the AuNP surface, which led to fluorescence recovery. To accurately determine DNA surface coverage on AuNPs, the DTTdisplaced, FAM-labeled DNA strands have been characterized by the calibration curves generated in solution (solution assay), or on the gel without SYBR Gold staining (gel electrophoresis assay 
without staining) or with (gel electrophoresis assay with staining). After overnight incubation at room temperature, the displaced DNA was separated from the AuNPs via centrifugation and the surface coverage of the NoX-FAM probe modified AuNP was determined as $\sim 48( \pm 5)$ DNA strands per particle using the solution assay based on the generated calibration curve of the unconjugated NoX-FAM probe in solution with the excitation at $495 \mathrm{~nm}$ and the emission at 520 nm (Figure 54A).

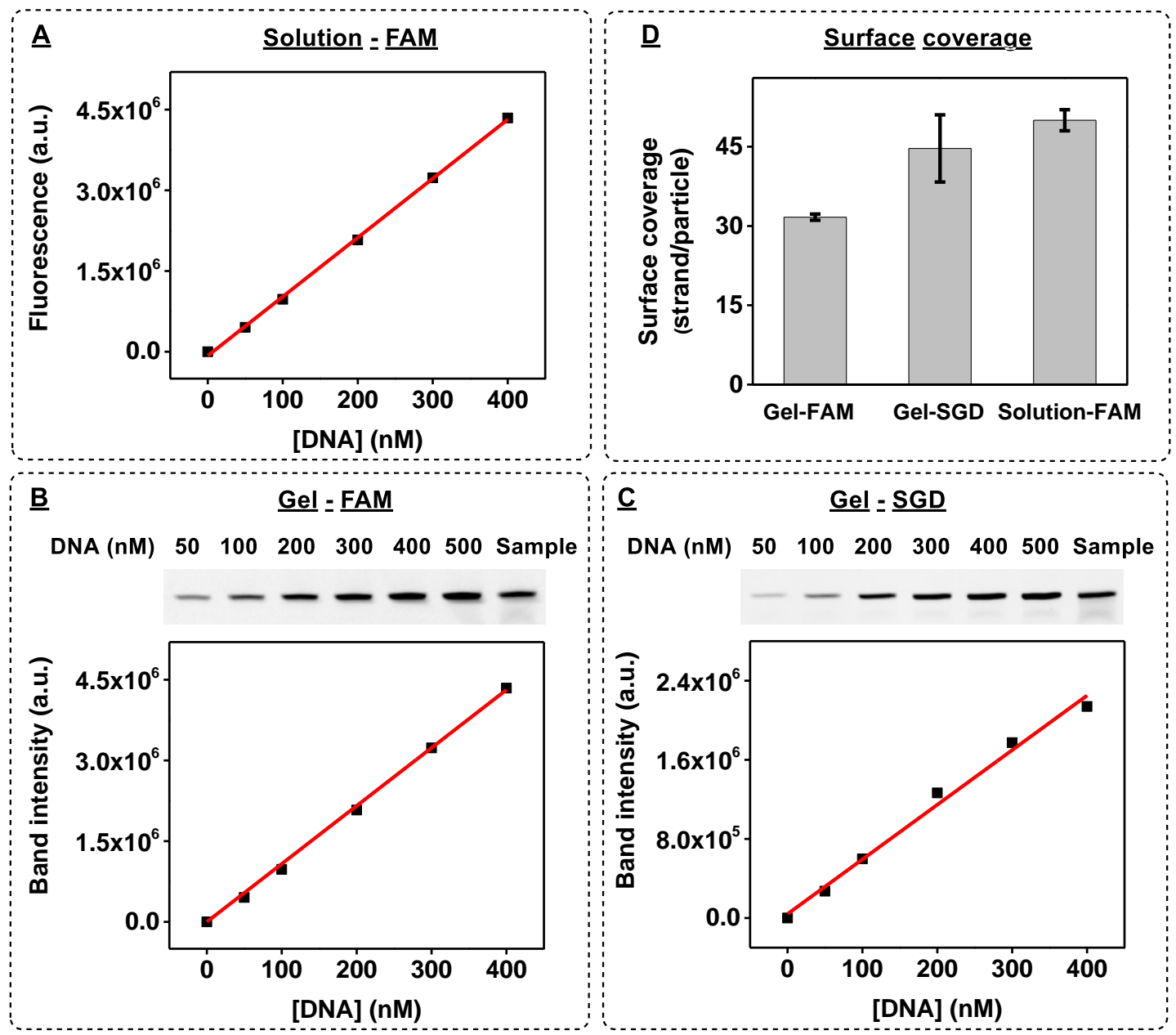

Figure 54. Characterization of the surface coverage of NoX-FAM DNA on AuNPs. (A) Calibration curve of the fluorescence intensity of NoX-FAM DNA $(0-400 \mathrm{nM})$ in solution assay. (B) Calibration curve of the gel image of NoX-FAM DNA $(0-400 \mathrm{nM})$ without staining. (C) Calibration curve of the gel image of NoX-FAM DNA $(0-400 \mathrm{nM})$ with $0.5 \times$ SYBR Gold (SGD) staining. (D) Comparison of the surface coverage using different assays. 
The samples were then loaded into the denatured PAGE with prepared standard calibration samples. Since 6-FAM emits fluorescence at $520 \mathrm{~nm}$ under UV light, we directly imaged the sample-loaded gel after the separation. Using the calibration curve generated with the standard concentrations of unconjugated FAM-labeled DNA on the gel, we obtained that the surface coverage of the NoX-FAM probe modified AuNP was $\sim 32( \pm 3)$ DNA strands per particle without staining (Figure 54B), which was slightly lower than the value obtained in the solution assay $(\sim 48( \pm 5)$ DNA per particle). The resultant gel was then stained with $0.5 \times$ SYBR Gold and the calculated surface coverage of the NoX-FAM probe modified AuNP was $41( \pm 3)$ DNA per particle with staining (Figure 54C), which is close to the values obtained from the solution assay.

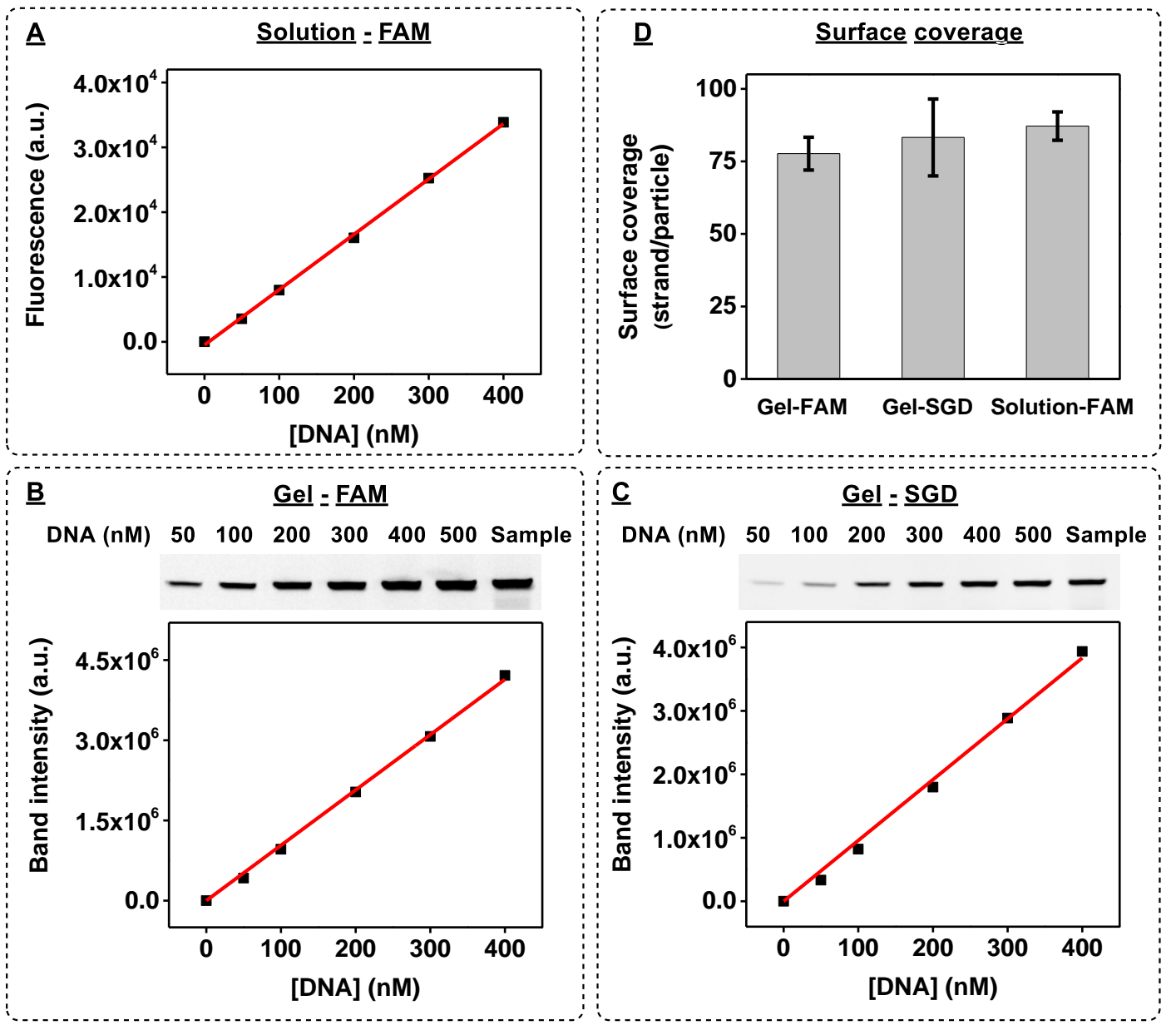

Figure 55. Characterization of the surface coverage of $1 X$-FAM DNA on AuNPs. (A) Calibration curve of the fluorescence intensity of 1X-FAM DNA $(0-400 \mathrm{nM})$ in the solution assay. (B) Calibration curve of the gel image of 1X-FAM DNA $(0-400 \mathrm{nM})$ without staining. (C) 
Calibration curve of the gel image of 1X-FAM DNA $(0-400 \mathrm{nM})$ with $0.5 \times$ SYBR Gold (SGD) staining. (D) Comparison of the surface coverage using different assays.
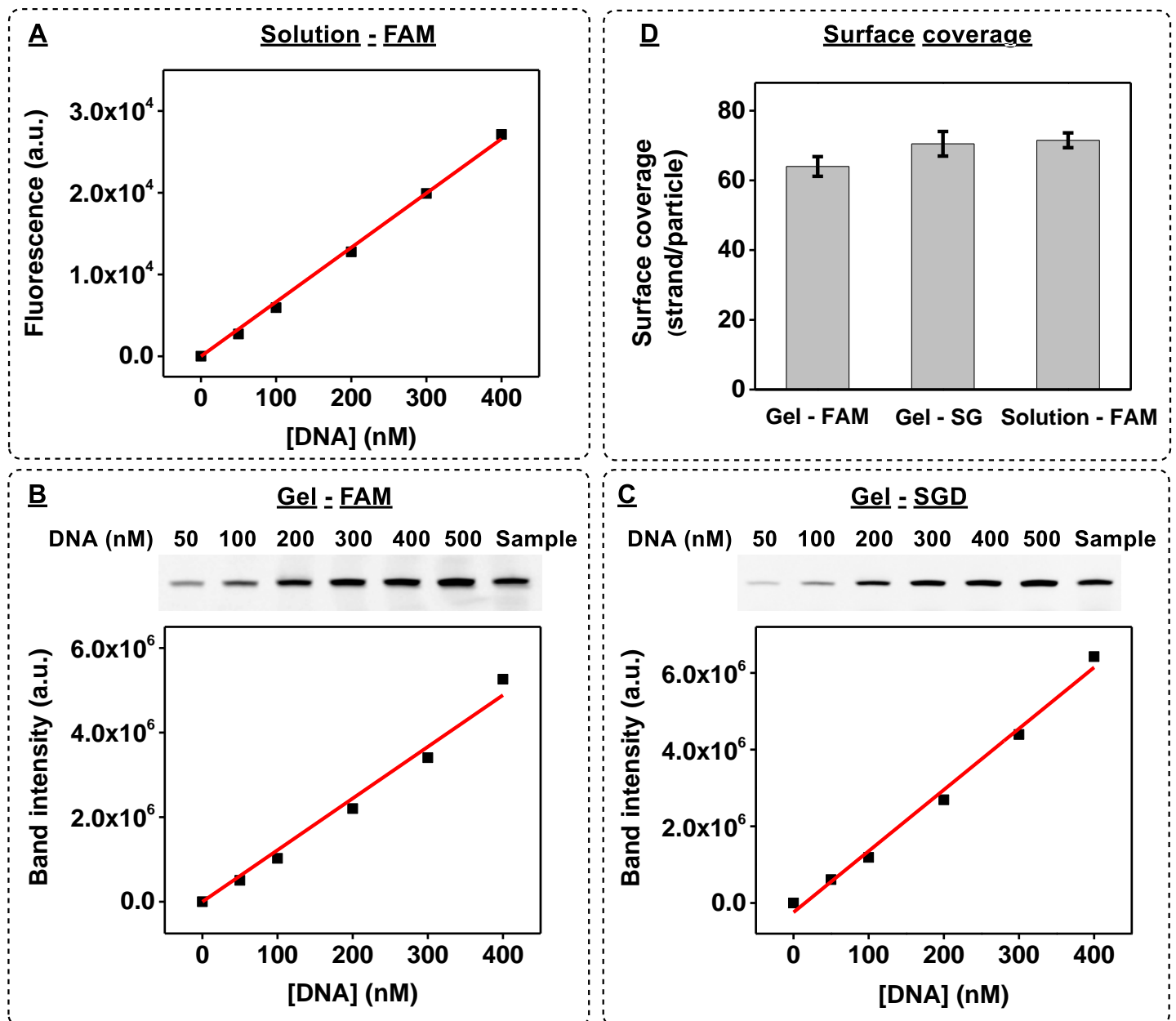

Figure 56. Characterization of the surface coverage of $2 X$-FAM DNA probe. (A) Calibration curve of the fluorescence intensity of 2X-FAM DNA $(0-400 \mathrm{nM})$ in the solution assay. (B) Calibration curve of the gel image of 2X-FAM DNA $(0-400 \mathrm{nM})$ without staining. (C) Calibration curve of the gel image of 2X-FAM DNA $(0-400 \mathrm{nM})$ with $0.5 \times$ SYBR Gold (SGD) staining. (D) Comparison of the surface coverage using different assays.

To test the effect of various DNA modifications on the methods' accuracy, we further modified AuNPs with other two thiolated DNA strands (1X-FAM and 2X-FAM DNAs, X stands for abasic site, $1 \mathrm{X}=$ one abasic site and $2 \mathrm{X}=$ two abasic sites) and determined the surface coverage of 1X-FAM and 2X-FAM DNAs modified AuNPs using the solution assay and the gel 
electrophoresis assay described above. Two calibration curves were respectively formed using the fluorescence intensity of the standard concentrations of unconjugated FAM-labeled DNA in solution (Figures 55A and 56A), the gel band intensity without (Figures 55B and 56B) or with SYBR Gold staining (Figures 55C and 56C). The results demonstrated that the DNA surface coverage obtained with SYBR Gold staining was consistent with the values determined by the solution assay. However, the surface coverage determined by the FAM fluorescence on the gel was lower for both DNA sequences (Figures 55D and 56D).

5.3.1.2 Characterization of DTT-displaced, unlabeled DNA stained with either SYBR Gold stained gel electrophoresis assay or OliGreen-based solution assay

The SYBR Gold stained gel electrophoresis assay was validated by the standard DTT displacement assay for the accurate determination of surface coverage of three FAM-labeled oligonucleotides on AuNPs. We further used the validated SYBR Gold stained gel electrophoresis assay to evaluate the performance of OliGreen based solution assay on measuring surface coverage of unlabeled DNA strands on AuNPs. Our experimental results demonstrated that the OliGreen based solution assay allowed an accurate determination of DNA surface coverage on AuNPs.

Table 3. The sequences of two unlabeled thiolated DNAs employed (from 5' to 3').

NoX No-T8: HS-TTT TTT ACC ACA TCA TCC ATA TAA CTG AAA GCC AAA CAG 1-X No-T8: HS-TTT TTT ACC ACA TCA TCC /X/TAT AAC TGA AAG CCA AAC AG ${ }^{*} / \mathrm{X} /$ denotes the abasic site (a propyl residue, Spacer- $\mathrm{C}_{3}$ )

Two unlabeled thiolated DNA strands (Table 3, NoX No-T8 and 1-X No-T8) were used to modify AuNPs and identify the surface coverage of each DNA strand on AuNPs using the SYBR Gold stained gel electrophoresis assay as well as the OliGreen-based solution assay. The SYBR 
Gold stained gel electrophoresis assay started with DTT displacement by mixing the unlabeled DNA-modified AuNPs with the equal volume of $1.0 \mathrm{M}$ DTT solution for 16 hours at room temperature. The displaced DNA was separated from the AuNPs via centrifugation and loaded into the denatured PAGE with the prepared standard calibration samples. After the gel electrophoresis was complete, the gel was stained with $0.5 \times$ SYBR Gold for 25 minutes and imaged using a ChemiDoc MP imaging system. The calibration curve generated with known concentrations of standards on the same gel allowed us to calculate DNA surface coverage of NoX-No-T8 and 1-X No-T8 on AuNPs. The surface coverage of NoX-No-T8 strand and 1-X-NoT8 was $46( \pm 3)$ and $91( \pm 5)$, respectively (Figures 57A and 57B).
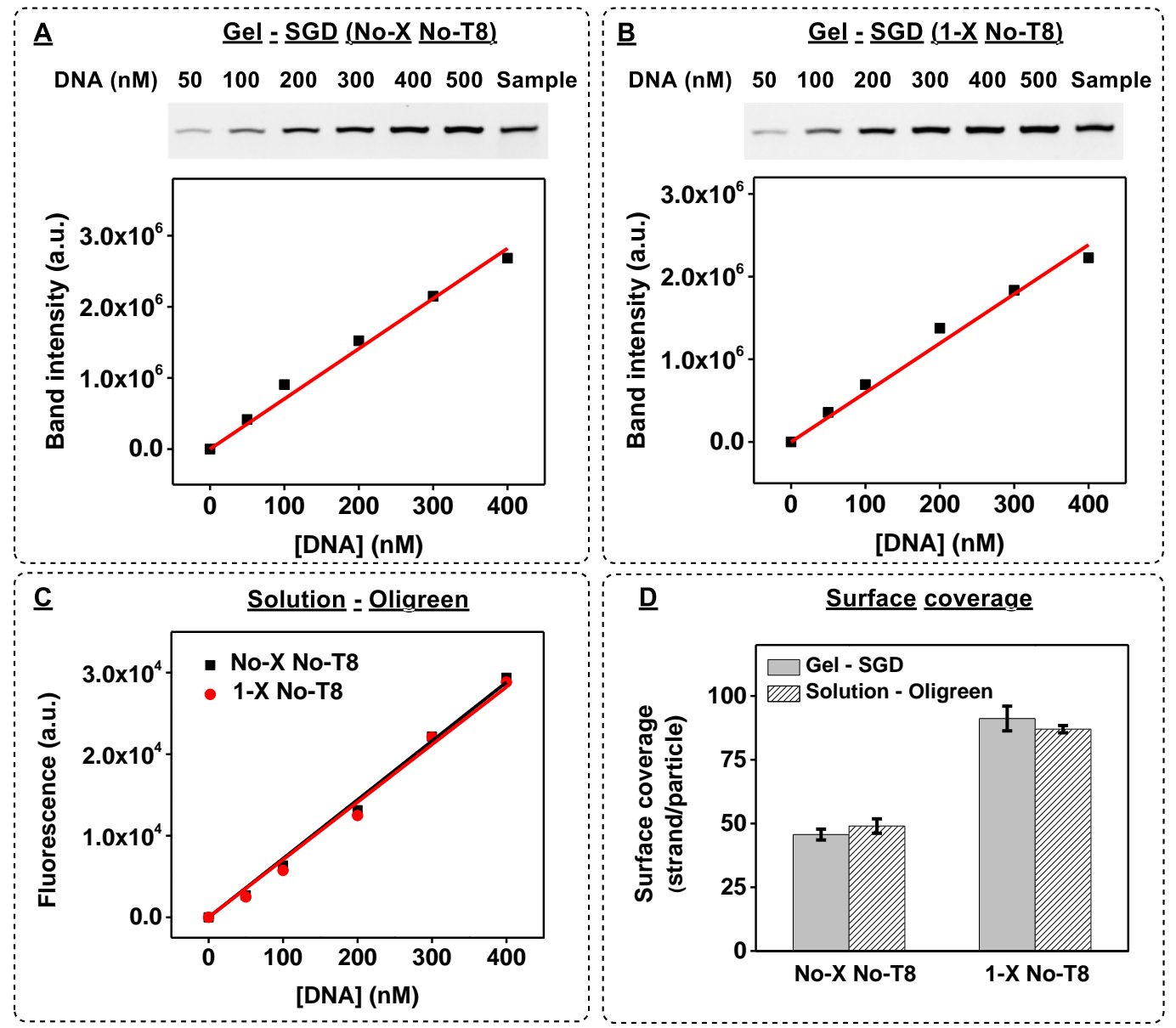

Figure 57. Characterization of the DNA surface coverage of NoX No-T8 DNA and 1-X No-T8 DNA. (A) Calibration curve of NoX No-T8 DNA $(0-400 \mathrm{nM})$ imaged with $0.5 \times$ SYBR Gold 
(SGD) staining. (B) Calibration curve of 1 X No-T8 DNA $(0-400 \mathrm{nM})$ imaged with $0.5 \times$ SYBR Gold (SGD) staining. (C) Calibration curve of NoX No-T8 DNA and 1-X No-T8 DNA (0 - 400 $\mathrm{nM}$ ) in the solution phase detected using OliGreen dye. (D) Comparison of the surface coverage using OliGreen based solution assay and SYBR Gold stained gel electrophoresis assay.

The displaced unlabeled DNA samples were also determined using a commercially available OliGreen-based, single-stranded oligonucleotide quantification kit. OliGreen dye is an ultrasensitive, fluorescent nucleic acid stain that is used for quantitating single-stranded DNA (ssDNA) in solution. OliGreen itself gives a very low fluorescence in solution. When it binds to nucleic acids, the fluorescence of OliGreen is significantly enhanced (Figure 58). Usually, the OliGreen fluorescent assay for ssDNA offers ultra-high sensitivity: a 1000-fold improvement in sensitivity over traditional absorbance methods. We used OliGreen to stain the displaced DNA and determined that AuNPs modified with No-X-No-T8 strand and 1X-No-T8 displayed 49( \pm 3$)$ and $87( \pm 5)$ oligonucleotides per particle, respectively (Figure 57C). We observed that the values obtained with OliGreen-based solution assay are highly consistent with the values from the SYBR Gold stained gel electrophoresis assay (Figure 57D).

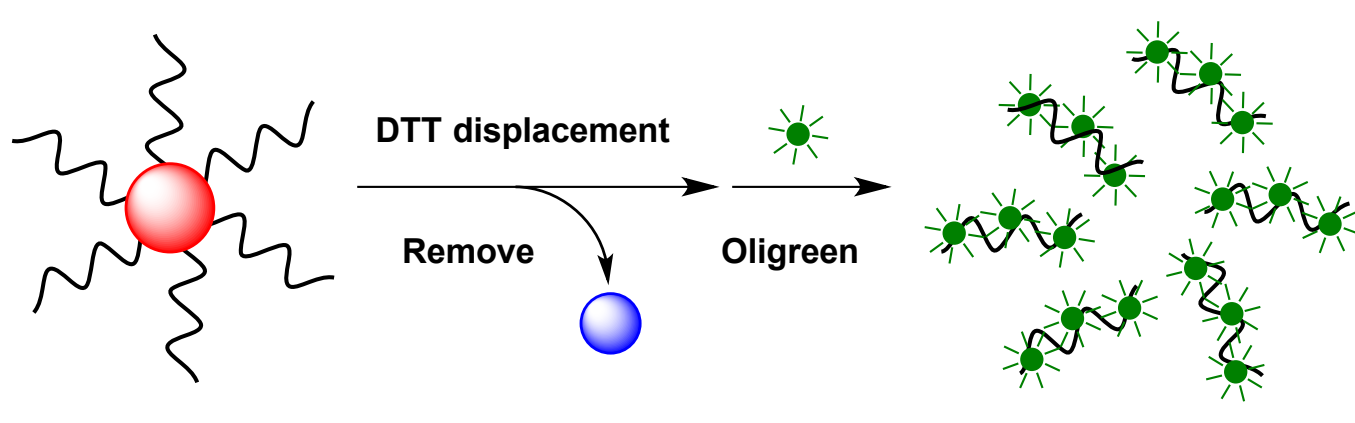

Unmodified DNA

Fluorescence on

Figure 58. Scheme of DTT displacement of AuNP-unlabeled DNA strands and the characterization of surface coverage of released DNA using OliGreen dye.

\subsubsection{Development of a simple assay based on ligand-exchange strategy to rapidly alter DNA surface coverage on AuNPs}

We recently developed an AuNP-based, Exo III-assisted target-recycled (EATR) colorimetric assay for DNA detection in a single tube with one set of DNA probe-modified AuNPs, a single 
exonuclease (Exo III), and the target DNA in question (Figure 59). ${ }^{70}$ The capture probe DNA which featured two propyl abasic sites were attached on AuNP via a thiol-gold bond at its 5' end. In the absence of perfectly matched (PM) target DNA, Exo III could not digest the surfaceattached single-stranded probe DNA and the solution remained red. In the presence of the target, the PM target formed a duplex with the immobilized probe DNA on the AuNP surface, with the $3^{\prime}$ poly $\left(\mathrm{T}_{8}\right)$ sequence of CP forming a sticky end. Exo III was inactive at this $3^{\prime}$ sticky end, ${ }^{285}$ and instead preferentially cleaved the match-flanked abasic sites through its apurinic endonucleolytic activity, converting the probe DNA into three nicked duplex fragments. The Exo III then recognized the newly-formed 3'-hydroxyl termini on the nicked ends of the probe fragments and rapidly catalyzed the stepwise removal of 5'-mononucleotides through its exonucleolytic activity. ${ }^{270,286}$ This ultimately sheared the probe fragments from the particle but released the PM strand intact. The released PM target was then recycled and hybridized with another probe DNA on the same or a different AuNP to begin the cycle anew. Ultimately, all of the probe DNAs were sheared from the AuNPs, leaving only short poly $\left(\mathrm{T}_{6}\right)$ linkers behind on the particles. The sheared AuNPs become unstable and undergo salt-induced aggregation in the reaction buffer, giving rise to a red-to-blue color change (Figure 59). Since a single DNA target can be recycled for many probe DNA-modified AuNPs, the signal was rapidly amplified with visible AuNP aggregation within 15 minutes under the optimized condition.

We found that the DNA surface coverage on the AuNP played a significant role in determining the reaction time required to achieve a clear red-to-blue change with maximum signal gain in the EATR-based colorimetric assay described above. To demonstrate the effect of DNA surface coverage on the reaction time, we designed a 5' thiolated 45-nt probe DNA (Table 3, named $1 \mathrm{X}$ No-T8 probe) containing an abasic site without any fluorophore label at the $3^{\prime}$ end. We first modified AuNPs that achieve a maximized DNA surface coverage. To do so, we prepared the DNA-modified AuNPs by incubating 15.8 nmole of tris (2-carboxyethyl) phosphine 
(TCEP)-reduced probe DNA with freshly prepared, citrate-coated AuNPs (59.2 picomoles) at room temperature with a DNA to AuNP ratio of 267:1. During the DNA modification, $\mathrm{NaCl}$ was gradually added into the solution (final concentration $\sim 0.3 \mathrm{M}$ ) to weaken the electrostatic interaction between the oligonucleotides in order to obtain a higher surface coverage. ${ }^{169}$ After the modification, we found that $25 \%$ DNA was unconjugated and the unbound DNA was further removed via centrifugation. The DNA-modified AuNPs were washed with $10 \mathrm{mM}$ Tris buffer ( $\mathrm{pH}$ 7.4). The surface coverage of the $1 \mathrm{X}$ No-T8 probe DNA-modified AuNPs was characterized by OliGreen-based solution assay, yielding a high surface density with 120 strands/particle.

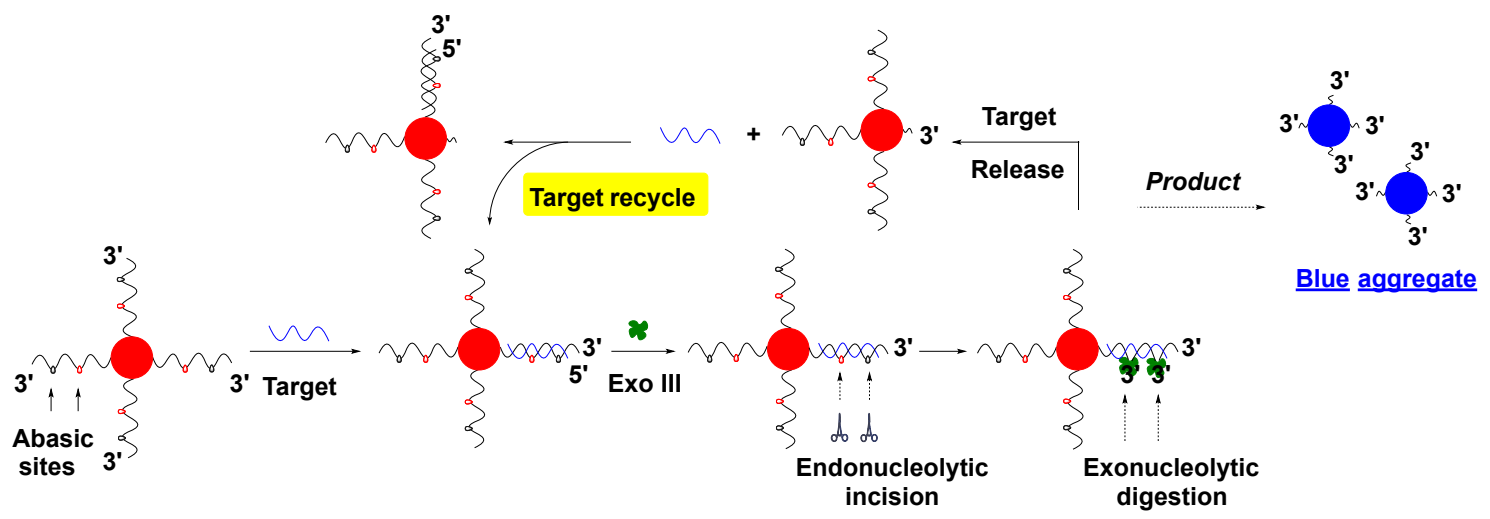

Figure 59. The AuNP-based, Exo III-assisted target recycling (EATR)-based colorimetric detection of DNA.

5.3.2.1 Using $\mathrm{MCH}$ to alter DNA surface coverage on AuNPs and testing Exo III activity on the MCH-treated DNA-modified AuNPs.

We then used the prepared 1X No-T8 probe DNA-modified AuNPs and a thiolated molecule - mercaptohexanol $(\mathrm{MCH})$ to develop a simple and rapid assay based on a ligand-exchange strategy to control the surface coverage of DNA-modified AuNPs. Based on the fact that MCH can reactively displace the immobilized probe DNAs from the gold surface (Figure 60), we incubated the $1 \mathrm{X}$ No-T8 probe DNA-modified AuNPs loaded with a maximal DNA surface 
coverage of 120 strands/particle with various concentrations of $\mathrm{MCH}(0-200 \mu \mathrm{M})$ for 30 minutes at room temperature to prepare seven batches of DNA-modified AuNPs with different surface coverage. After separating the MCH-treated AuNPs from the supernatant, the AuNP pellets were re-suspended in $10 \mathrm{mM}$ Tris buffer ( $\mathrm{pH}$ 7.4). We then mixed these seven batches of MCH-treated AuNPs with an equal volume of 1.0 M DTT and incubated the mixture at room temperature for 16 hours at room temperature to completely remove $1 \mathrm{X}$ No-T8 probe DNAs from the surface, after which the samples were centrifuged. The supernatant was collected and the fluorescence was measured after mixing with $1 \times$ OliGreen dye. We then established a calibration curve by recording the fluorescence intensity of mixtures containing different concentrations of unlabeled, unconjugated 1X No-T8 probe DNA and $1 \times$ OliGreen dye prepared with the same buffer condition (Figure 61A). We determined that the DNA-modified AuNPs adjusted with the MCH concentration of $0,10,25,50,75,100$ and $200 \mu \mathrm{M}$ displayed $120( \pm 6), 60( \pm 4), 50( \pm 4), 40( \pm$ $3), 36( \pm 3) 30( \pm 3)$ and $25( \pm 2)$ oligonucleotides per particle, equivalent to surface coverage of $37.5( \pm 1.9), 18.8( \pm 1.3), 15.6( \pm 1.3), 12.5( \pm 1), 11.3( \pm 1), 9.4( \pm 1)$ and $7.8( \pm 0.6) \mathrm{pmole} / \mathrm{cm}^{2}$ respectively (Figure 61B).
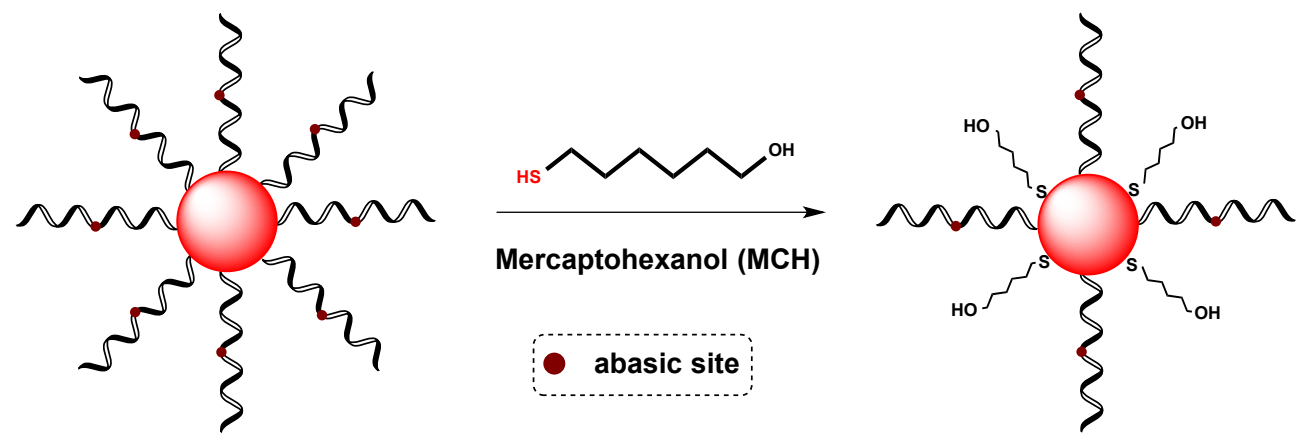

Figure 60. Scheme of regulating DNA surface coverage on AuNP by using mercaptohexanol $(\mathrm{MCH})$.

We found that $\mathrm{MCH}$ can effectively regulate DNA surface coverage on AuNPs, and it was found as the MCH concentration increases, the DNA surface coverage on the AuNPs decreases. 
When the MCH concentration was higher than $200 \mu \mathrm{M}$, the MCH-treated AuNPs were not stable in the reaction buffer due to a poor protection from very few probe DNAs retained on the surface.

A

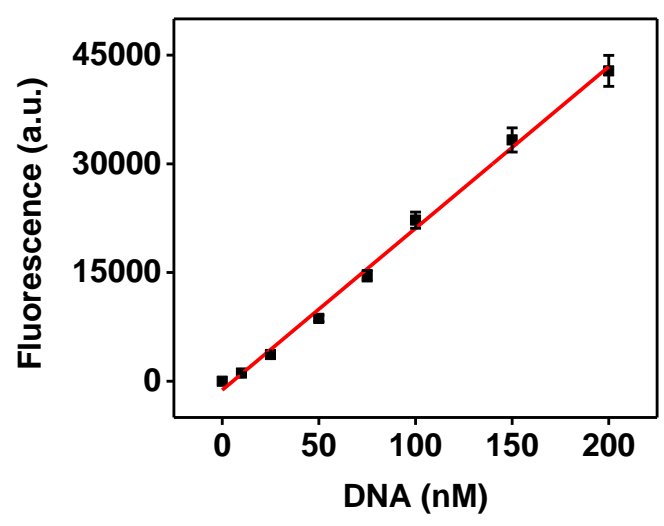

B

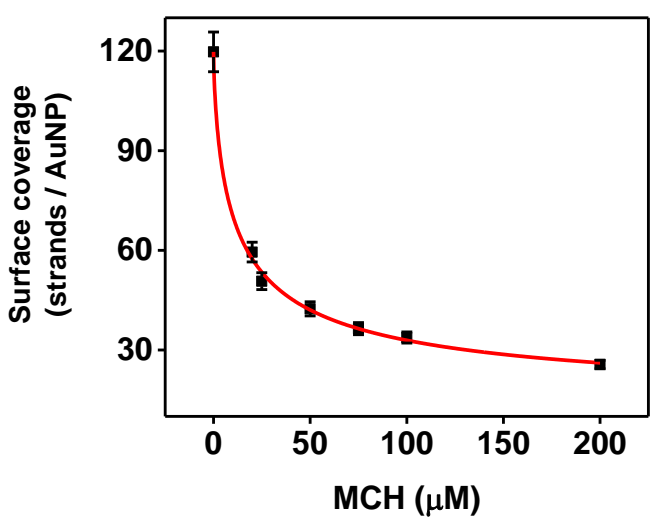

Figure 61. Effect of MCH concentration on the adjusted DNA surface coverage. (A) Calibration curve with 1X No-T8 standard. (B) DNA surface coverage regulated by different concentrations of $\mathrm{MCH}$.
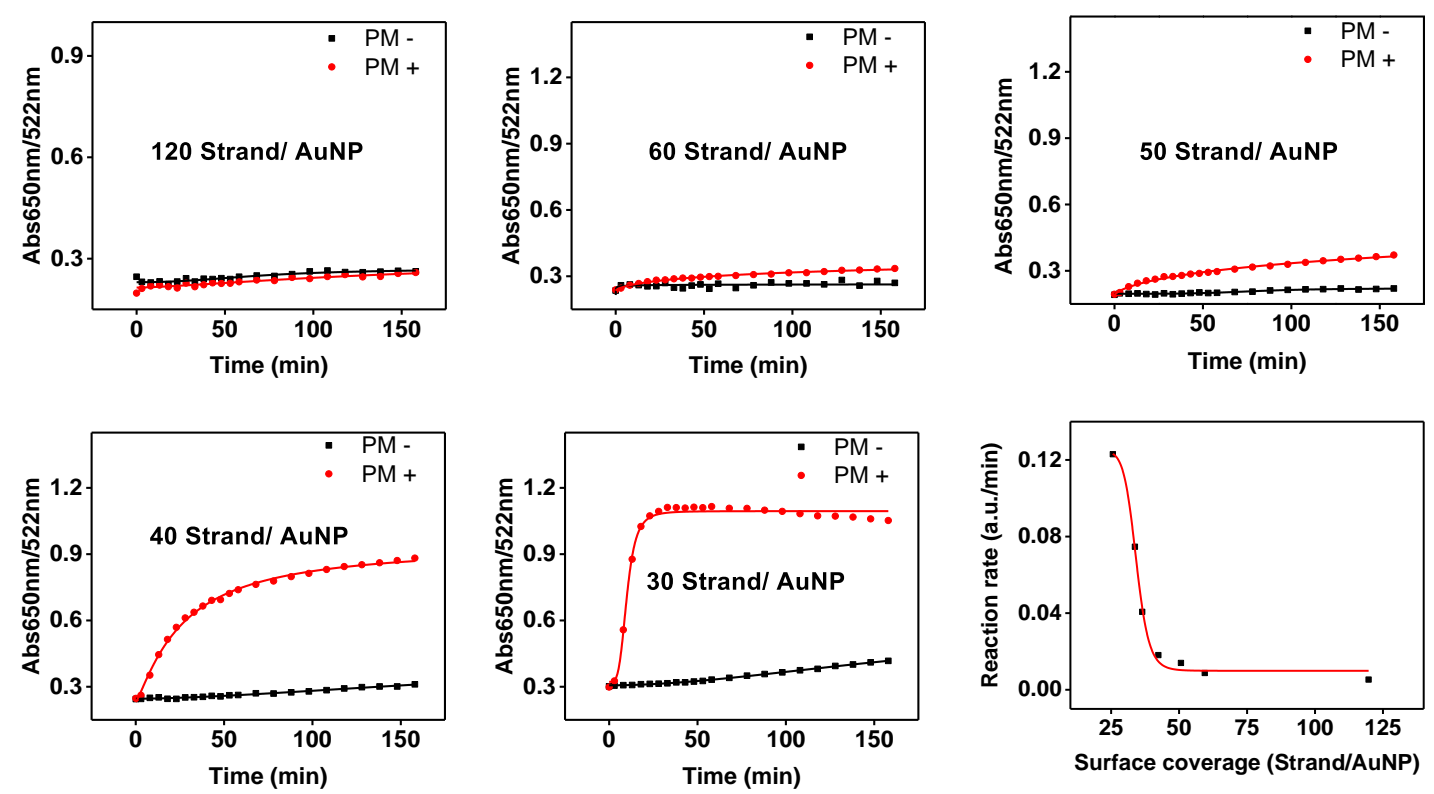

Figure 62. Effect of Exo III activity on the MCH-regulated DNA surface coverage. The Exo III digestion on AuNP-DNA was performed with or without of $100 \mathrm{nM}$ perfectly matched DNA target in $10 \mathrm{mM}$ Tris buffer solution containing $20 \mathrm{mM} \mathrm{NaCl}$ and $6 \mathrm{mM} \mathrm{MgCl} 2$ and $1 \mathrm{U}$ of Exo III. 
These MCH-treated, DNA-modified AuNPs were stable in the reaction buffer and the solution was red in color. However, when the immobilized probe DNAs were hybridized with its PM target, Exo III facilitated the digestion on the formed duplexes and rapidly sheared the DNA probes from the particles. The sheared AuNPs became unstable in the reaction buffer and underwent salt-induced aggregation, giving rise to a red-to-blue color change. We observed that the MCH-regulated, DNA-modified AuNPs perform a much faster Exo III-assisted AuNP aggregation than these non-treated, DNA-modified AuNPs (Figure 62). Specifically, we resuspend the MCH-displaced AuNPs in $10 \mathrm{mM}$ Tris buffer containing $20 \mathrm{mM} \mathrm{NaCl}$ and $6 \mathrm{mM}$ $\mathrm{MgCl}_{2}$. We collected the time-dependent UV-vis measurements of these MCH-treated AuNP samples in the presence of $1 \mathrm{U}$ Exo III and $100 \mathrm{nM}$ PM target (Figure 62), and used the ratio of absorbance at $650 \mathrm{~nm}$ to $522 \mathrm{~nm}\left(\mathrm{~A}_{650 \mathrm{~nm}} / \mathrm{A}_{522 \mathrm{~nm}}\right)$ as an indicator to measure the aggregation degree of AuNPs. The colorimetric result confirmed that the DNA surface coverage gave a significant effect on the Exo III-assisted aggregation rate of the DNA-modified AuNPs. Clearly, the reaction rate of the surface-based enzymatic reaction was increased by $\mathrm{MCH}$-reduced surface coverage and the DNA-modified AuNPs with a lower surface coverage produced the fastest aggregation rate (Figure 62). We believe that the DNA-modified AuNPs with a lower surface coverage provided more space and less charge repulsion to facilitate target DNA hybridization and Exo III digestion on the particle surface, resulting in a much shorter reaction time. In contrast, the high DNA surface coverage usually had a steric effect and charge repulsion on target DNA hybridization and salt-accumulated inhibition on enzyme activity, producing a slow reaction or no reaction within a fixed measurement time.

5.3.2.2 Using DTT to alter DNA surface coverage on the DNA-modified AuNPs and testing Exo III activity on the DTT- treated DNA-modified AuNPs

Alternatively, we employed another thiolated molecule, dithiothreitol (DTT), to adjust the DNA surface coverage on the AuNPs through ligand-exchange reaction. DTT is much more 
reactive toward the gold surface and can bind AuNPs via two thiol groups to effectively displace the immobilized DNA strands from the gold surface (Figure 63).
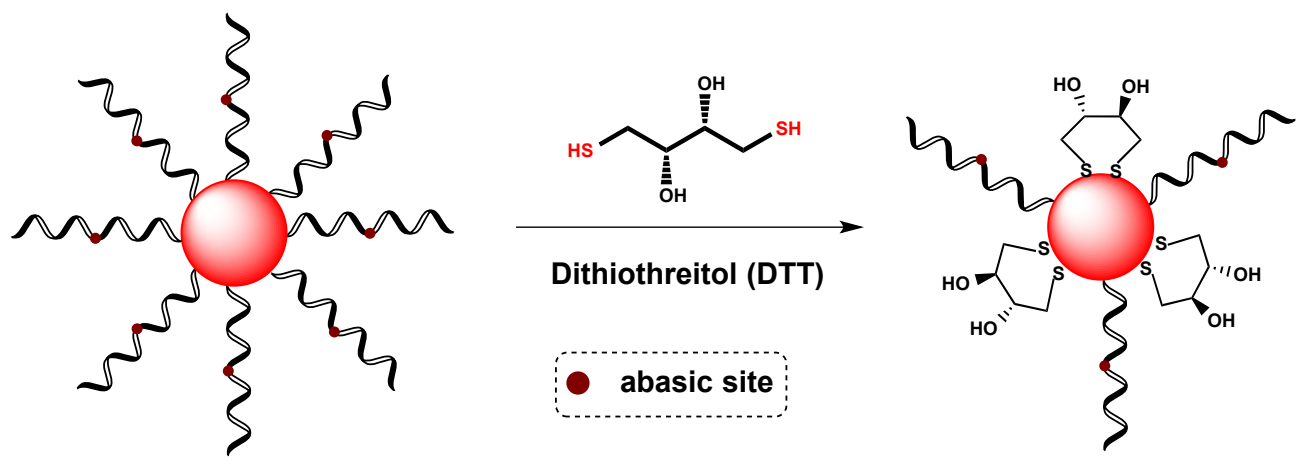

Figure 63. Scheme of adjusting DNA surface coverage on AuNP by using dithiothreitol (DTT).

DTT displacement was carried out in $10 \mathrm{mM}$ Tris buffer (pH 7.4) using different concentrations of DTT $(0-200 \mu \mathrm{M})$ for 30 minutes at room temperature. The displaced DNA and excess DTT were separated from the DNA-modified AuNPs via centrifugation. To characterize the surface coverage, the DTT-adjusted AuNPs were re-suspended in $10 \mathrm{mM}$ Tris buffer (pH 7.4) and then mixed with an equal volume of $1.0 \mathrm{M} \mathrm{DTT}$ for 16 hours at room temperature to completely displace all DNA strands immobilized on the AuNP. The supernatant was collected, and the fluorescence was measured after mixing with $1 \times$ OliGreen dye. The calibration curve was generated by using the known concentrations of unconjugated $1 \mathrm{X}$ No-T8 DNA and $1 \times$ OliGreen dye under the same buffer conditions (Figure 64A). We determined that the DNA-modified AuNPs adjusted with the DTT concentration of 0,10, 25, 50, 75, 100 and $200 \mu \mathrm{M}$ displayed 120 $( \pm 8), 70( \pm 7), 60( \pm 5), 50( \pm 6), 40( \pm 3), 38( \pm 4)$ and $30( \pm 4)$ oligonucleotides per particle, equivalent to surface coverage of $37.5( \pm 2.5), 21.9( \pm 2.2), 18.8( \pm 1.6), 15.6( \pm 1.9), 12.5( \pm 0.9)$, $11.9( \pm 1.2)$ and $9.4( \pm 1.2)$ pmole $/ \mathrm{cm}^{2}$, respectively (Figure 64B). We found that the DTT-treated AuNPs were not stable in the reaction buffer due to poor protection from very few probe DNAs retained on the surface when the DTT concentration was higher than $200 \mu \mathrm{M}$. The calculated 
surface coverage indicated that the surface coverage on the DTT-adjusted, DNA-modified AuNPs decreased as the DTT concentration increased (Figure 64B).

A

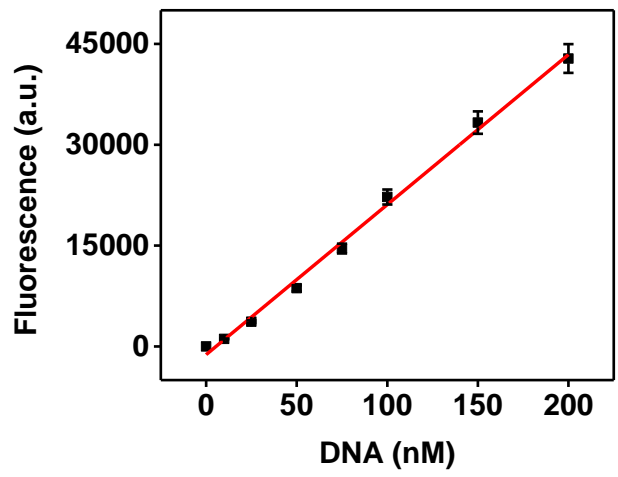

B

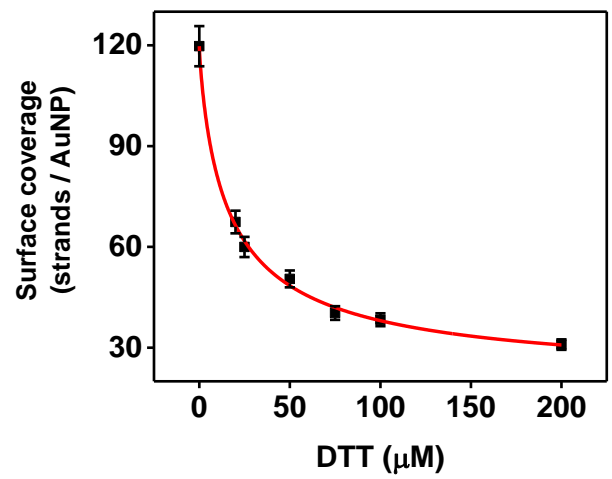

Figure 64. Effect of DTT concentration on the regulated DNA surface coverage. (A) Calibration curve with 1X No-T8 standard. (B) DNA surface coverage regulated by different concentrations of DTT.
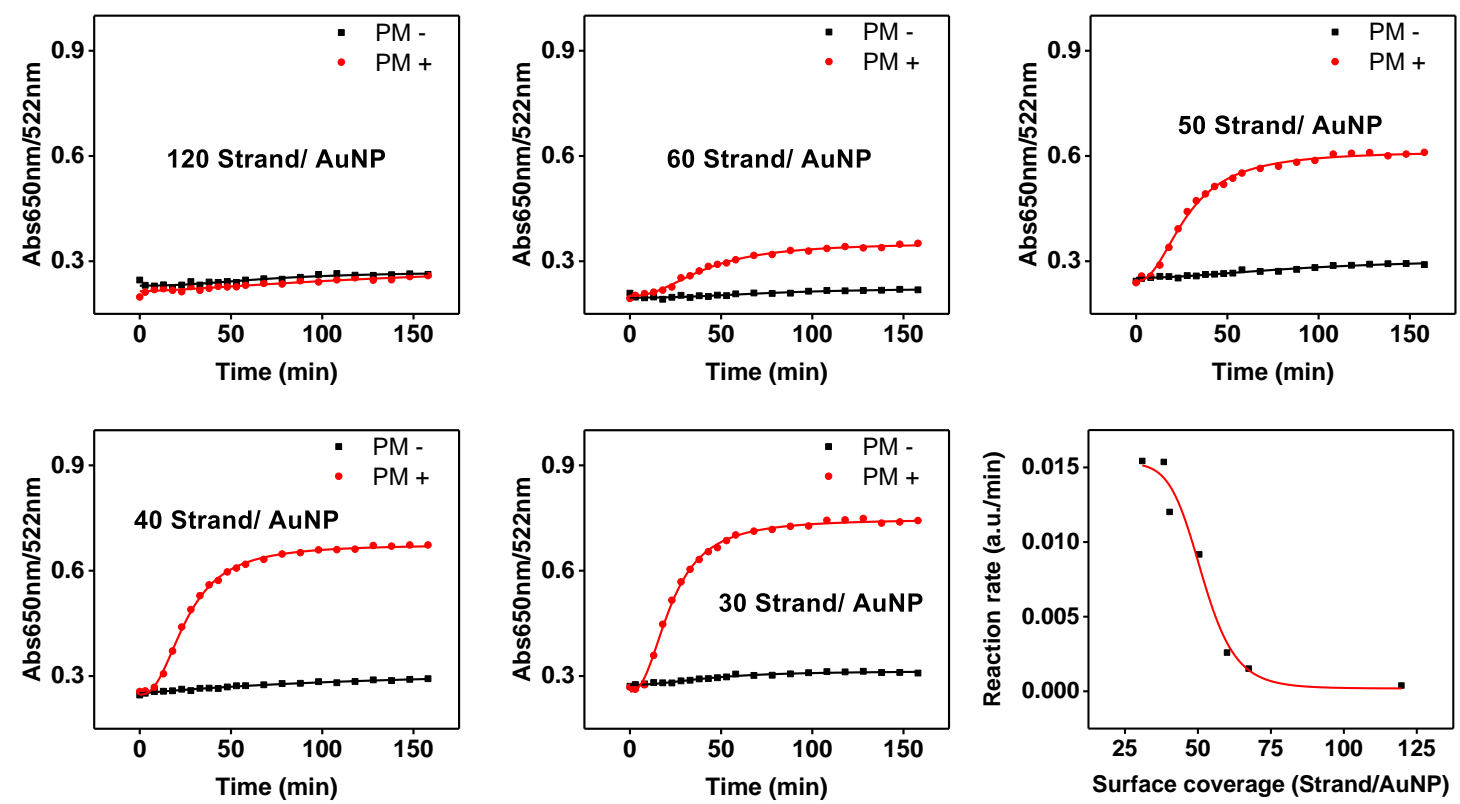

Figure 65. Effect of Exo III activity on the DTT-regulated DNA surface coverage. The Exo III digestion on AuNP-DNA was performed with or without of $100 \mathrm{nM}$ target DNA in $10 \mathrm{mM}$ Tris buffer solution contains $20 \mathrm{mM} \mathrm{NaCl}$ and $6 \mathrm{mM} \mathrm{MgCl} 2$ and $1 \mathrm{U}$ of Exo III. 
The DTT-treated, DNA-modified AuNPs also demonstrated a higher activity for Exo III digestion than those non-treated, DNA-modified AuNPs. We performed Exo III reaction with these seven batches of DTT-treated DNA-modified AuNPs in $10 \mathrm{mM}$ Tris buffer solution containing $20 \mathrm{mM} \mathrm{NaCl}, 6 \mathrm{mM} \mathrm{MgCl} 2$, $1 \mathrm{U}$ of Exo III and $100 \mathrm{nM}$ PM target. The ratio of $\mathrm{A}_{650 \mathrm{~nm}} / \mathrm{A}_{522 \mathrm{~nm}}$ collected during the time course clearly demonstrated that the reaction rate increased as the DNA surface coverage of the modified-AuNPs decreased (Figure 65).

We have developed a simple and rapid assay to adjust the DNA surface coverage of DNAmodified AuNP by incubating the as-prepared DNA-modified AuNPs loaded with the maximum amount of DNA with low concentrations of $\mathrm{MCH}$ or DTT for 30 minutes at room temperature. The surface-based DNA hybridization and enzyme reaction can be strongly affected by DNA surface coverage. The significant effect of DNA surface coverage on the assay performance was confirmed by using our AuNP-based, EATR-based colorimetric assay.

5.3.3 Development of a colorimetric detection of cocaine based on Exo III-inhibited digestion on target-aptamer complex

Table 4. DNA sequences for CBSA (from 5' to 3').

38GC: GGG AGA CAA GGA AAA TCC TTC AAC GAA GTG GGT CTC CC

38GC-LF: CTC CTT CAA CGA AGT GGG TCT CAA AAA AAA AA

38GC-SF: TTT TTG AGA CAA GGA G

5325-10A-LF: CTCCTTCAACGAAGTGGGTTCCTTCAACGAAGTGGGTCTC

AАAAAAAAAA

5325-6A-LF: CTCCTTCAACGAAGTGGGTTCCTTCAACGAAGTGGGTCTCAAAAAA

5325-5A-LF: CTCCTTCAACGAAGTGGGTTCCTTCAACGAAGTGGGTCTCAAAAA

5325-4A-LF: CTCCTTCAACGAAGTGGGTTCCTTCAACGAAGTGGGTCTCAAAA

5325-3A-LF: CTCCTTCAACGAAGTGGGTTCCTTCAACGAAGTGGGTCTCAAA

5325-AT-SF: TTTTTGAGACAAGGAACAAGGAG

5325-XT-SF: TTTTTGAGACAAGG/iSpC3/ACAAGGAG

SH-5325-6T-SF: SHTTTTTTGAGACAAGG/iSpC3/ACAAGGAG

${ }^{*} / \mathrm{iSpC}_{3} /$ denotes the abasic site (a propyl residue, Spacer- $\mathrm{C}_{3}$ ) 
To test the effect of DNA surface coverage on small molecule detection, we have developed an AuNP-reported colorimetric assay for cocaine detection based on Exo III inhibition on the target-aptamer complex. We have found that Exo III preferentially digested the unbound folded aptamer whereas such digestion was inhibited for target-aptamer complexes. Specifically, the digestion of Exo III was performed with a newly engineered cocaine-binding aptamer (38-GC), ${ }^{287}$ which pre-folded into a three-way junction with a blunt end at 3' end. In the absence of cocaine, Exo III catalyzed 3'-to-5' digestion of 38-GC by the stepwise removal of mononucleotides to form short, single-stranded products due to its preferentially recognized blunt termini (Figure 66, no cocaine). In the presence of cocaine, however, 38-GC underwent a subtle shift of a few nucleotides within the three-way junction involved with cocaine binding. We observed that Exo III was highly sensitive to this minor reorganization within the binding domain, and was no longer capable of digesting the cocaine-bound aptamer (Figure 66, with cocaine). Importantly, Exo III's structure selectivity was sufficiently strong to prevent recognition of aptamer complexes formed via weaker interactions with other, non-target interferent molecules.

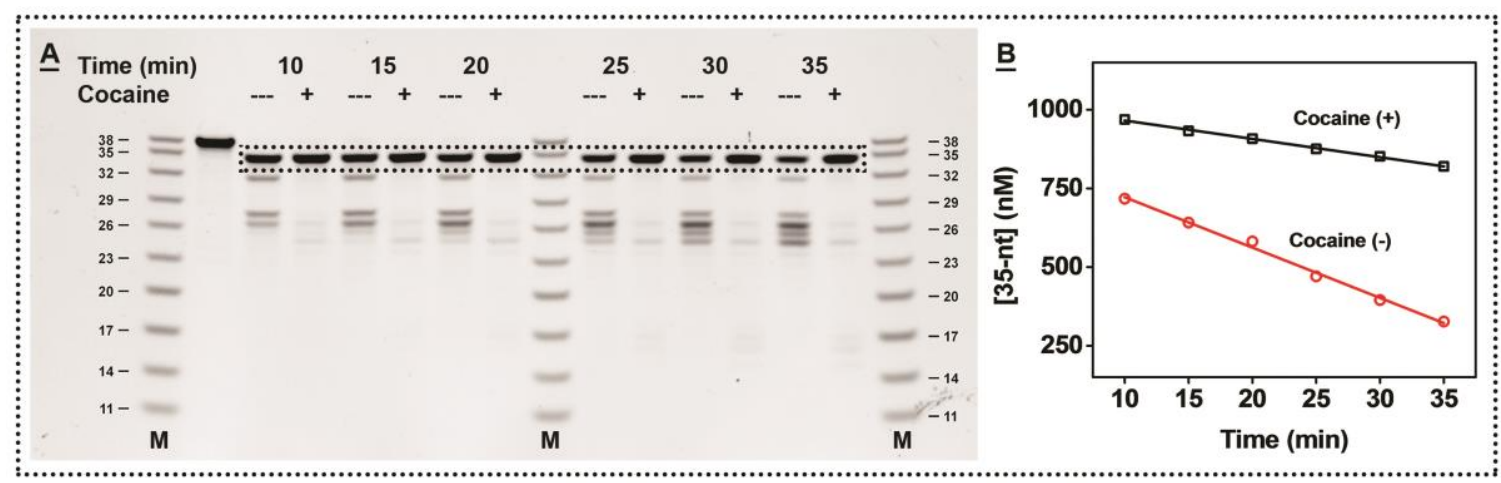

Figure 66. Time-course of 38-GC digestion with Exo III in the absence or presence of cocaine. (A) PAGE analysis of digested products. (B) Extent of 35-nt product degradation over time in the presence or absence of cocaine. [38GC]: $1 \mu \mathrm{M}$, [cocaine]: $250 \mu \mathrm{M}$, [Exo III]: $0.26 \mathrm{U} / \mu \mathrm{L}$. 


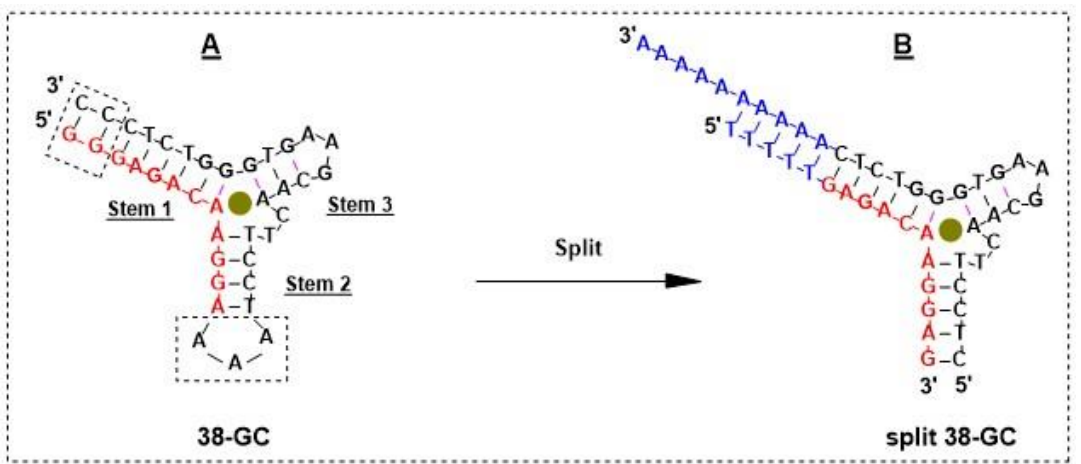

Figure 67. Engineering split aptamer 38-GC from its single cocaine aptamer.

Based on the Exo III inhibition on target-bound single-stranded cocaine-binding 38-GC aptamer, we engineered a pair of 38-GC split aptamers (Figure 67) in order to obtain an enhanced target-induced signal and test Exo III inhibition on the 38-GC split aptamer. 38-GC contained a three-way junction with the target-binding domain located at its center, surrounded by three double-stranded stems (stems 1, 2 and 3) and two loops (AAG and AAA loops) (Figure 67A). We have determined that stem 3 is essential for cocaine binding, while stem 1 and stem 2 both contribute to the stability of the three-way junction structure that forms upon target binding. First, the AAA loop of 38-GC (Figure 67A) was removed to form an open end (Figure 67B). Second, two G-C base-pairs in the stem 1 were replaced with five A-T base pairs (Figure 67B) in order to increase the stability of aptamer-cocaine complex at room temperature. ${ }^{288}$ The 38 -GC split aptamer included a long fragment (38GC-LF) and a short fragment (38GC-SF). A Poly(A) $)_{5}$ were added to the 3 ' end of the 38GC-LF to prevent nonspecific Exo III digestion.

We used the 38-GC split aptamer to test the Exo III inhibition on the aptamer-cocaine complex. The reaction was performed in $10 \mathrm{mM}$ Tris buffer ( $\mathrm{pH} 7.4)$ containing $125 \mathrm{mM} \mathrm{NaCl}$ and $0.1 \mathrm{mM} \mathrm{MgCl}_{2}$. Exo III (10 U) were added into the 38 -GC split aptamer solution with or without $250 \mu \mathrm{M}$ cocaine to perform the digestion and the reaction was stopped by mixing $5 \mu \mathrm{L}$ sample collected at different reaction time with $10 \mu \mathrm{L}$ formamide loading buffer $(71.25 \%$ formamide, $10 \%$ glycerol, $0.125 \%$ SDS, $25 \mathrm{mM}$ EDTA, and $0.15 \%$ (w/v) bromophenol blue and 
xylene cyanol). The digested products collected at different reaction times were characterized via $15 \%$ denaturing polyacrylamide gel electrophoresis (PAGE) (Figure 68A), $3 \mu \mathrm{L}$ of each sample mixture was loaded into each well. Separation was carried out at $20 \mathrm{~V} / \mathrm{cm}$ for 3 hours in $0.5 \times$ TBE running buffer. The gel was stained with $0.5 \times$ SYBR Gold solution for 25 minutes and imaged using a ChemiDoc MP imaging system (Bio-Rad).

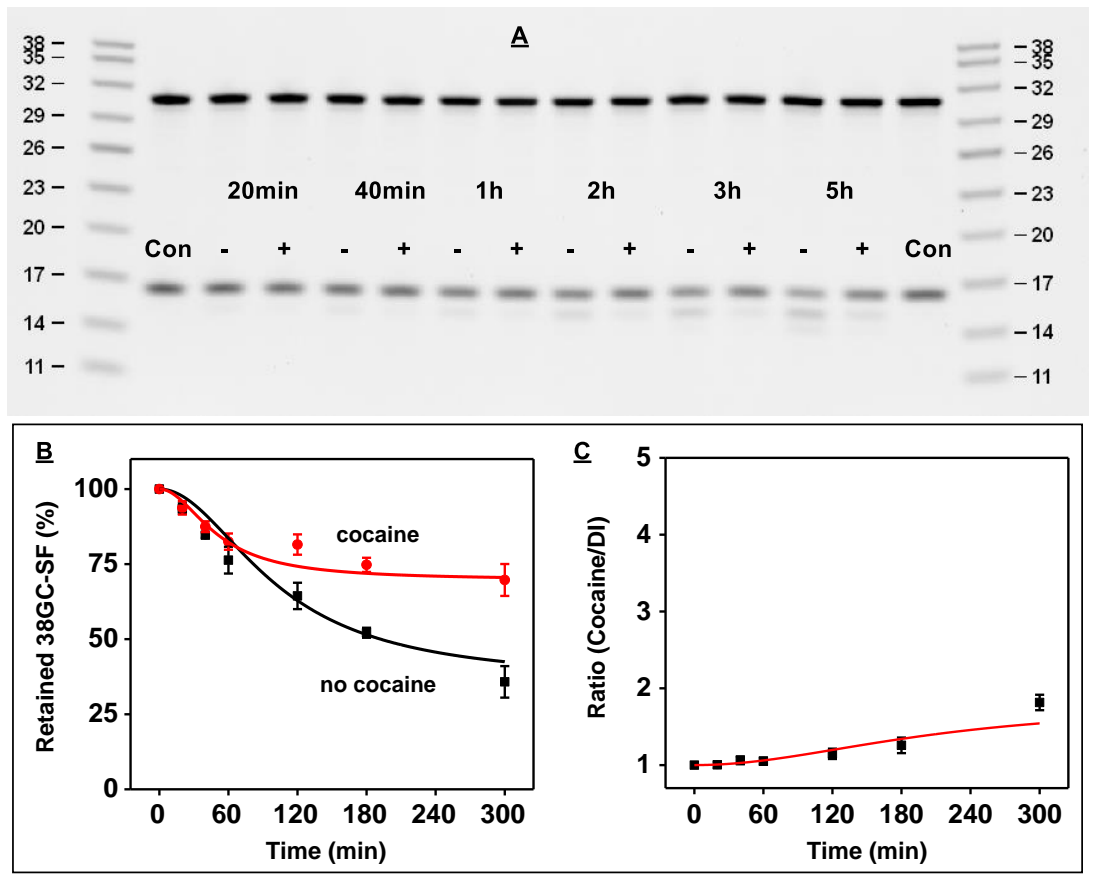

Figure 68. Exo III digestion on split 38-GC with and without $250 \mu \mathrm{M}$ cocaine in Tris buffer $(\mathrm{pH}$ 7.4) including $125 \mathrm{mM} \mathrm{NaCl}$ and $0.1 \mathrm{mM} \mathrm{MgCl} 2$.

We found that most of the 38GC-SF remained intact in the first one hour and a slight cocaineresulted inhibition of Exo III was observed after 2 hours (Figure 68B). A very small amount of 15-nt and 14-nt major products were formed by the Exo III removal of one or two nucleotides from the 3' end. We did not observe any further digestion of the newly-formed products due to the Exo III inhibition on the aptamer-cocaine complex. More of the 38GC-SF were digested as the time increased: after a 5-hour digestion, $42 \%$ and $75 \%$ of the $38 \mathrm{GC}-\mathrm{SF}$ remained in the absence and presence of $250 \mu \mathrm{M}$ cocaine, respectively. The calculated ratio of the remained 38SF reached a maximum ratio of only two after 5 hours (Figure 68C). Clearly, only a small amount 
of the 38-GC split aptamer underwent a target-induced assembly to form the preferred duplexed substrates for Exo III digestion upon addition of cocaine.

To increase the target-binding affinity of the split 38-GC aptamer, we engineered a tandem split cocaine-binding aptamer with two target binding domains that can bind two cocaine molecules cooperatively (termed as cooperative binding split aptamer, CBSA) (Figure 69). In biology, cooperativity comes from the fact that binding of the first molecule changes the binding constant of the second molecule, while the term "cooperativity" in our system has been borrowed to describe the requirement of two molecules working together to enhance the target-binding affinity of the split aptamer. Cooperative target binding of split aptamers requires the incorporation of at least two binding domains into a single pair of fragments. We began with our previously-reported cocaine-binding aptamer 38-GC (Figure 69A) and generated two different pairs of split aptamer fragments based on 38-GC, in which stem 3 remained intact but the 3' end of stem 1 was truncated and the AAA loop of stem 2 was eliminated (Figure 69B). We subsequently generated a construct from these two sets of split aptamers, in which stem 1 from one set was linked to stem 2 from the second set (Figure 69C). The resulting CBSA consisted of a short fragment (CBSA-SF) and a long fragment (CBSA-LF), in which the fragments remain separated in the absence of target. We anticipated that these two fragments would form two tandem cocaine-binding domains when fully assembled with cocaine (Figure 69D). The resulting CBSA-5325 aptamer construct contained four complementary base-paired segments within the target/aptamer complex. In the absence of cocaine, the long (5325-10A-LF) and short (5325-ATSF) fragments were expected to remain separated (Figure 70A). Upon addition of cocaine, the CBSA fragments underwent cooperative target-induced assembly and form aptamer-cocaine complex (Figure 70B). 

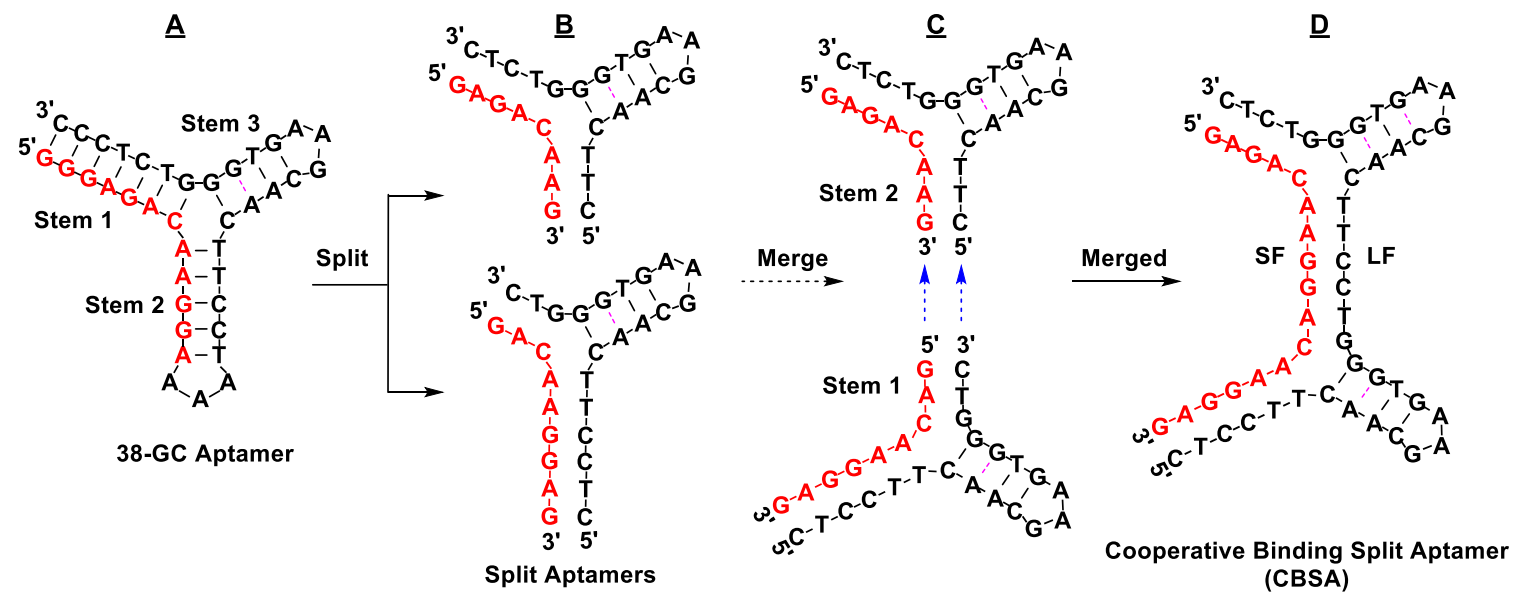

Figure 69. The design of cocaine-binding CBSA. The sequence of 38-GC (A) was truncated to form two split aptamer pairs (B). Stem 1 of one set of split aptamer was merged with stem 2 of the other set of split aptamer (C) to form an engineered CBSA (D) comprising a short fragment (SF) and a long fragment (LF).

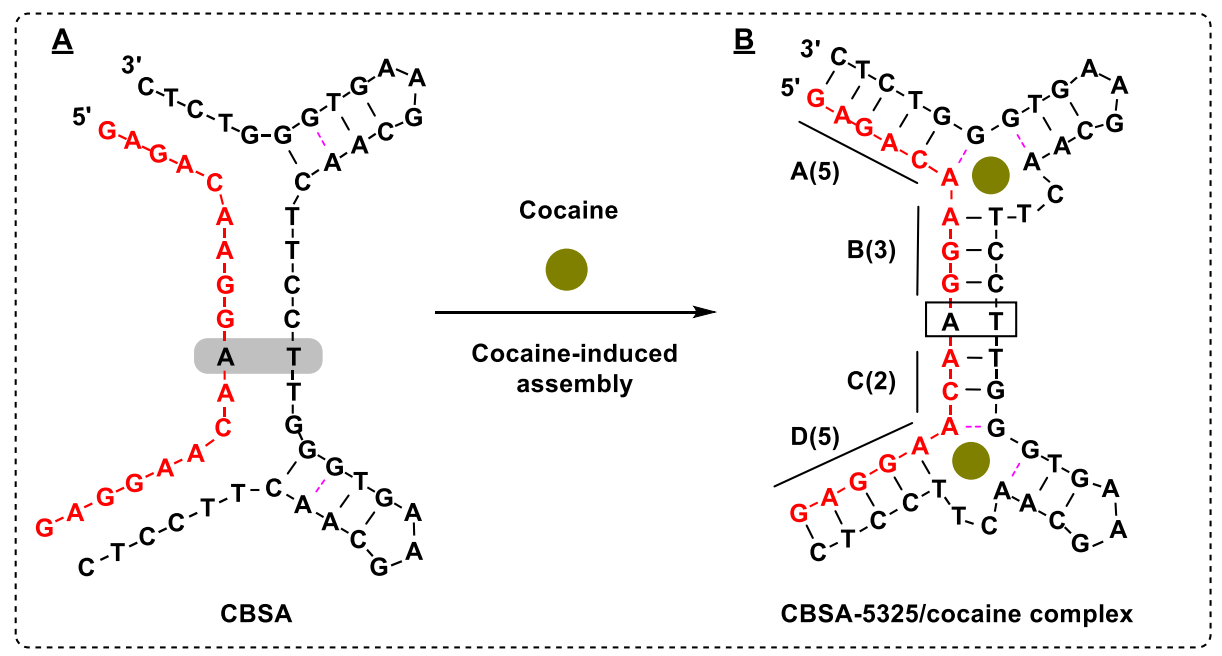

Figure 70. Structure of the cocaine unbound and bound CBSA-5325-AT aptamer.

We performed Exo III digestion of CBSA-5325 under optimized condition and found that $40 \%$ and $80 \%$ 5325-AT-SF DNA respectively remained in the absence and presence of $250 \mu \mathrm{M}$ cocaine after a 5-hour digestion (Figure 71B). We observed the removal of one or two nucleotides from the 3' end of 5325-AT-SF and did not observe any further digestion of the formed products (Figure 71A), which was possible due to the Exo III inhibition on the aptamer-cocaine complex. 
The calculated ratio of the remained 38-SF in the absence and presence of cocaine was still small even after a 5-hour digestion (Figure 71C).
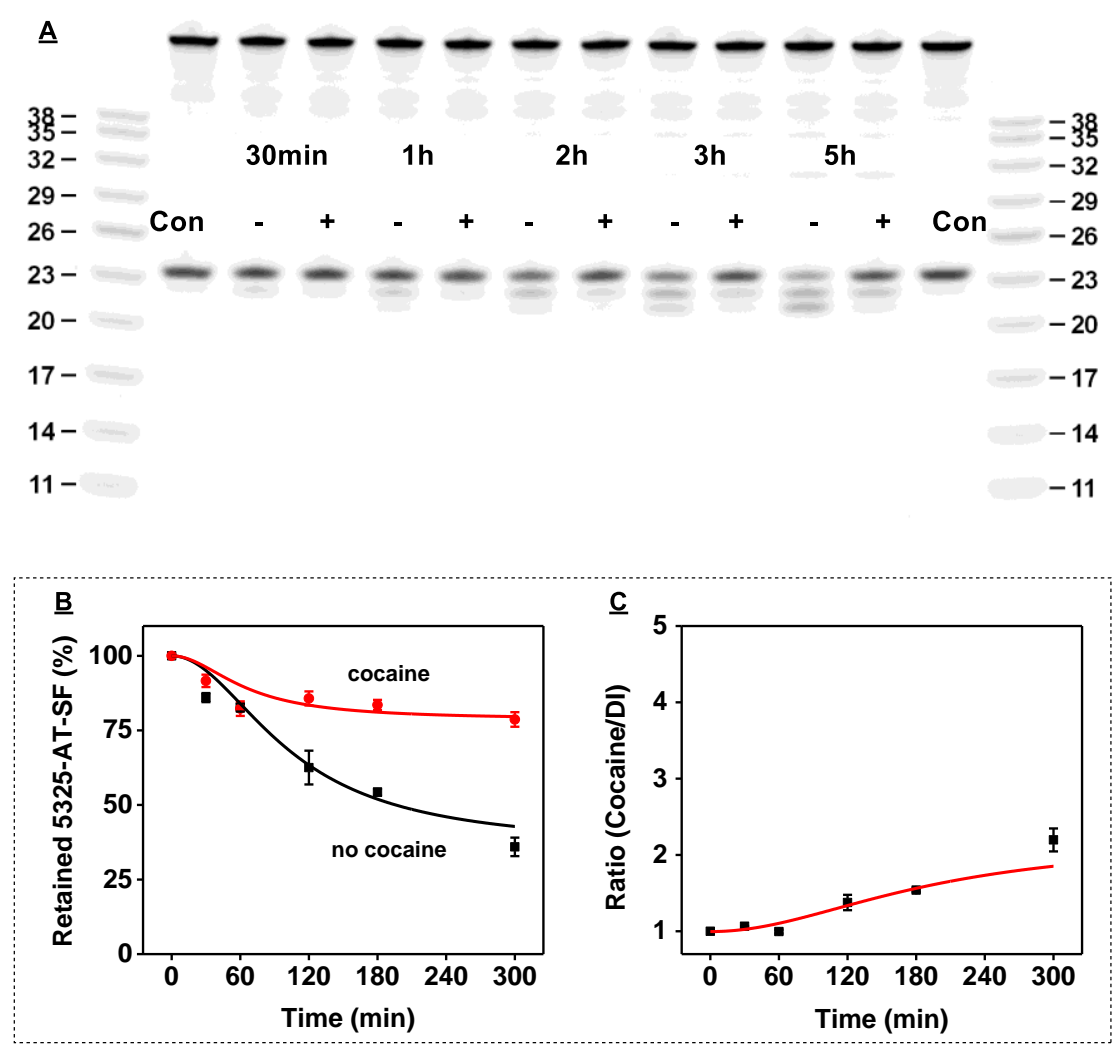

Figure 71. Exo III digestion on CBSA 5325-AT with and without $250 \mu \mathrm{M}$ cocaine in Tris buffer ( $\mathrm{pH} 7.4$ ) including $125 \mathrm{mM} \mathrm{NaCl}$ and $0.1 \mathrm{mM} \mathrm{MgCl}_{2}$.

To increase the selectivity of Exo III inhibition on aptamer-cocaine complex, we modified the 5325-AT-SF by replacing the adenosine (at position 9 from 3') of the short fragment with a C3 spacer (5325-XT-SF) to form an AP site with a thymine in the opposite position within the long fragment upon cocaine binding (Figure 72). The long (5325-10A-LF) and short (5325-XT-SF) fragments were used to test Exo III digestion under the same buffer condition, and the digested products were characterized by using $15 \%$ denature PAGE. Experimental results demonstrated that the long CBSA fragment remained intact due to its protected 3' end, and the CBSA short fragment was cleaved by Exo III's apurinic endonucleolytic activity at AP site, forming a 14-nt digestion product (Figure 73A). We found the cleavage of Exo III at AP site was greatly inhibited 
in the presence of cocaine; almost $100 \%$ of the short fragment was cleaved in the absence of the cocaine after a 20 -minute reaction. In contrast, $75 \%$ of 5325 -XT-SF remained intact in the presence of $250 \mu \mathrm{M}$ cocaine (Figure 73B). Clearly, the incorporation of the abasic site into the engineered CBSA increased both target selectivity and enzyme reaction speed (Figure 73C).

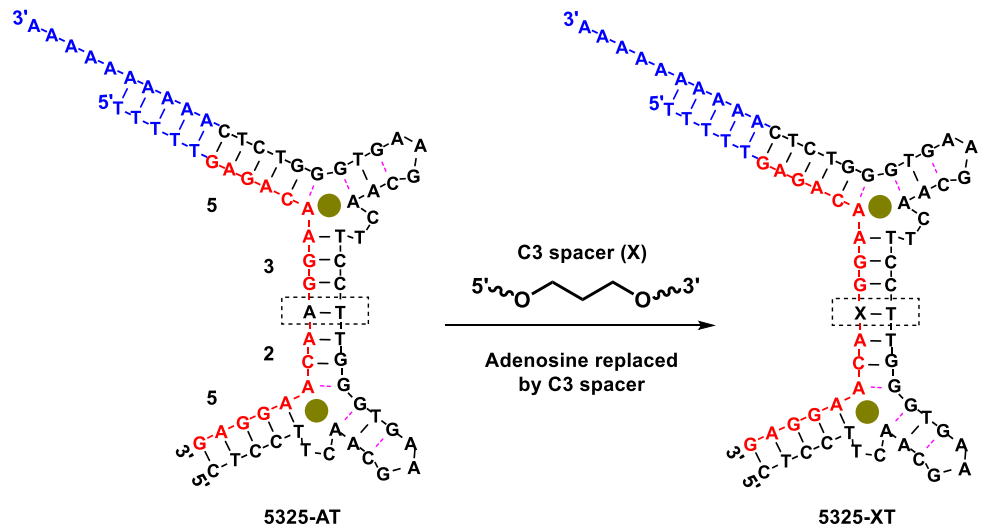

Figure 72. Engineering CBSA 5325-XT aptamer ("X" stands for abasic site) from CBSA 5325AT.

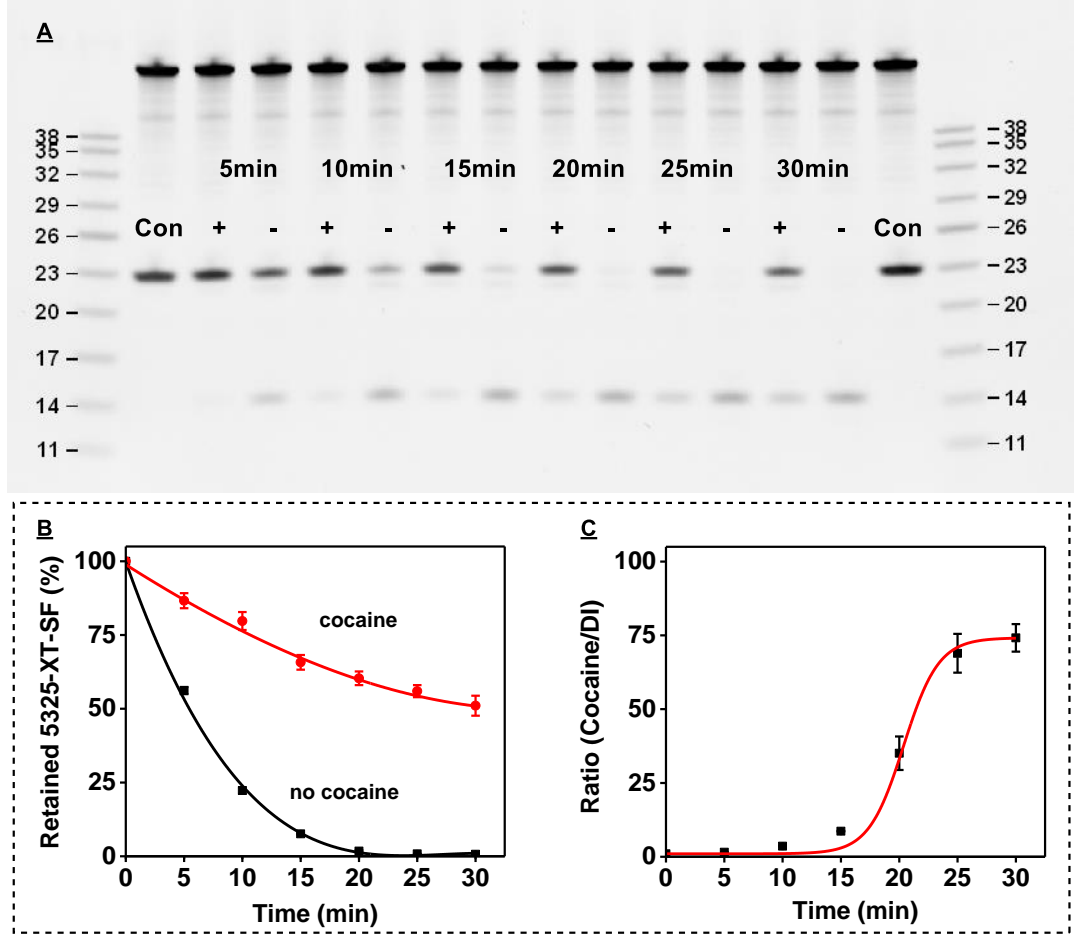

Figure 73. Exo III digestion on CBSA 5325-XT with and without $250 \mu \mathrm{M}$ cocaine in Tris buffer (pH 7.4) including $125 \mathrm{mM} \mathrm{NaCl}$ and $0.1 \mathrm{mM} \mathrm{MgCl}$. 
Based on the confirmed Exo III inhibition on CBSA aptamer-cocaine complex, we have employed CBSA-5325-XT to develop an AuNP-reported, Exo III-inhibited assay for colorimetric detection of cocaine (Figure 74). Specifically, thiolated 5325-XT-SF is modified onto freshly prepared AuNP via its 5' thiol group (Figure 74A), the 5' poly (T) 6 bases locate on 5325-XT-SF are used to form several "A-T" base-pairs with the 3' poly(A) located on 5325 long fragments to extend the recognition of the incorporated AP site away from the AuNP surface to improve aptamer fragment assembly and Exo III digestion. The CBSA long fragment is freely dispersed in the solution. In the presence of cocaine, AuNP-conjugated CBSA short fragment hybridizes with CBSA long fragment to form the aptamer-target complex (Figure 74B), Exo III's apurinic endonucleolytic activity is inhibited on the target-aptamer complex (Figure 74C). These undigested CBSA short fragments protect the modified AuNPs against salt-induced aggregation, and the solution stays red (Figure 74D).

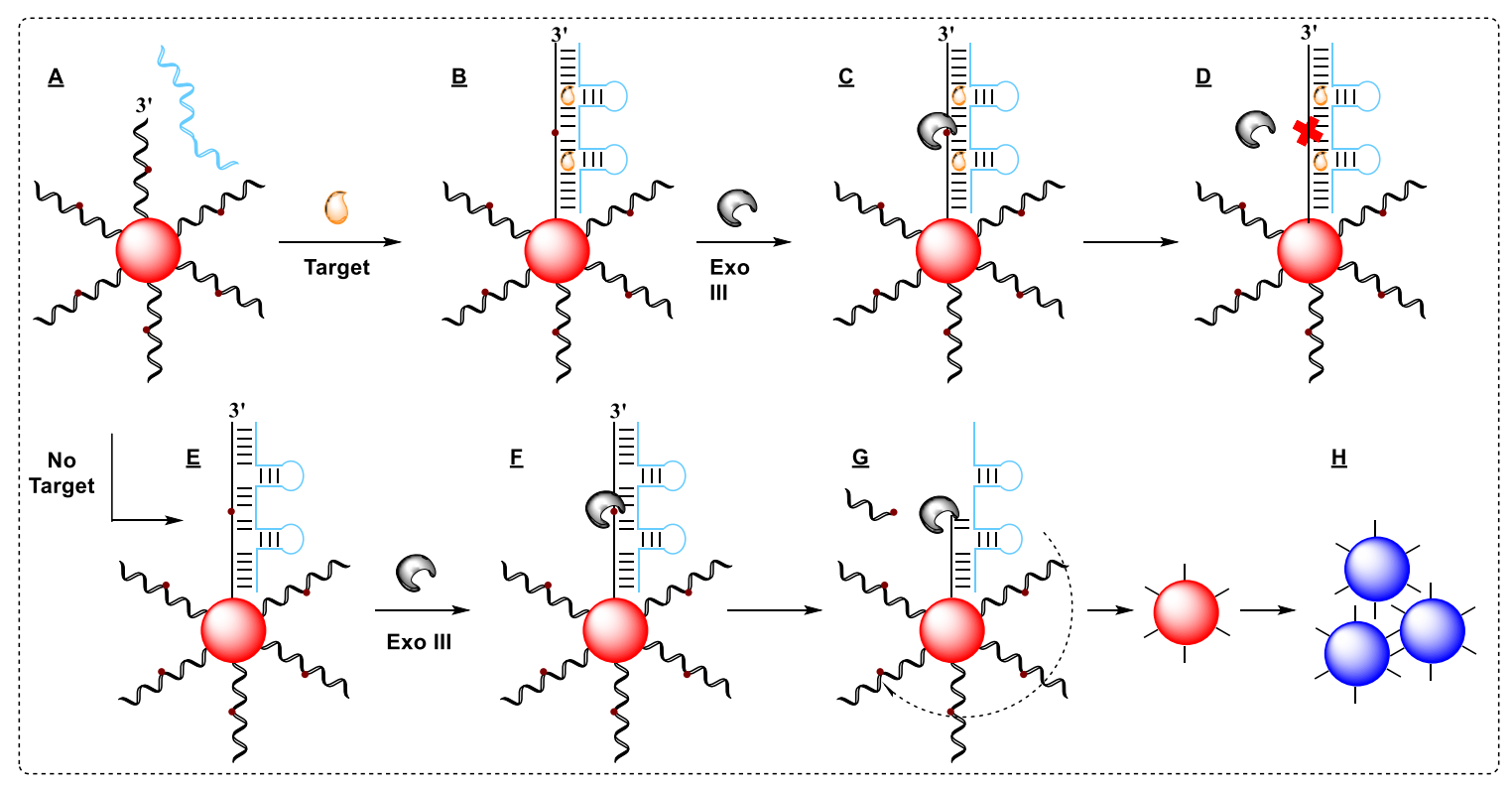

Figure 74. Working principle of AuNP-reported, Exo III-inhibited colorimetric detection of cocaine. 
In the absence of cocaine, Exo III is active on the duplex formed between the AuNPconjugated CBSA short fragment and the free CBSA long fragment (Figure 74E), cleaving the duplexed AP site and converting the short fragment into two separated binding domain (14- and 8-nt). These duplex fragments are dissociated at room temperature (Figure 74F), releasing the long fragment intact and cocaine from the AuNP surface. The released cocaine and long fragment are then recycled, and hybridize with another CBSA short fragment on the same or a different AuNPs to begin the cycle anew (Figure 74G). Ultimately, all of the CBSA short fragments are sheared from the AuNPs. The sheared AuNPs are unstable in the reaction buffer and undergo saltinduced aggregation, giving rise to a red-to-blue color change in the absence of cocaine (Figure 74H).
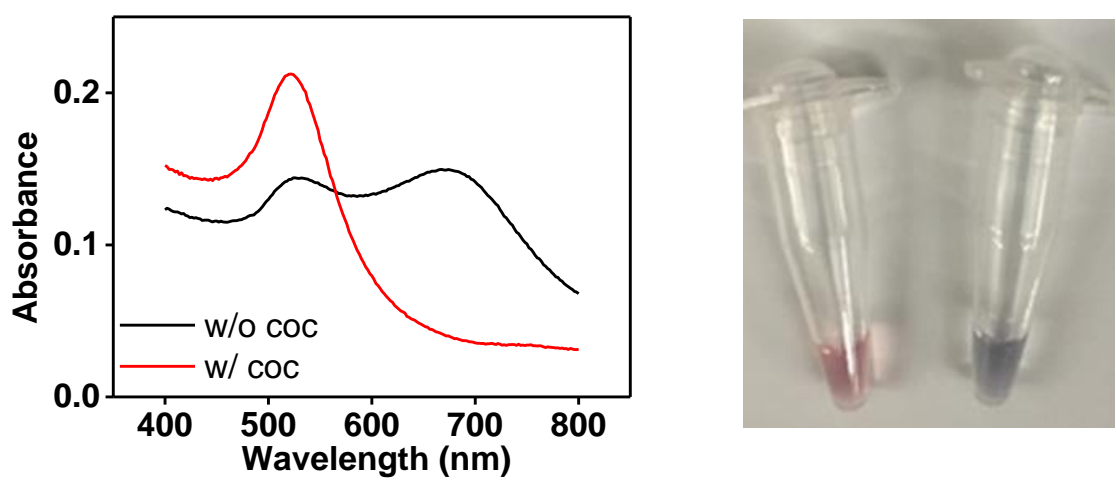

Figure 75. Proof of concept of AuNP-reported, Exo III-inhibited colorimetric detection of cocaine. (A) UV-vis spectra of the CBSA solution in the absence and presence of $250 \mu \mathrm{M}$ cocaine. (B) A visible blue color change observed in the presence of cocaine.

We then experimentally confirmed Exo III inhibition on CBSA-cocaine complex via an AuNP-aggregated signal. The colorimetric detection of cocaine was performed in $25 \mu \mathrm{L}$ of reaction buffer contains $10 \mathrm{mM}$ Tris buffer ( $\mathrm{pH}$ 7.4) containing $125 \mathrm{mM} \mathrm{NaCl}, 0.1 \mathrm{mM} \mathrm{MgCl} 2,1$ $\mu \mathrm{M}$ CBSA long fragment, $100 \mathrm{nM}$ 5325-XT-SF-modified AuNPs and $10 \mathrm{U}$ Exo III, with or without cocaine $(250 \mu \mathrm{M})$ at room temperature. We observed that the absorbance peak of well dispersed, DNA-modified AuNPs at $522 \mathrm{~nm}$ did not change when cocaine was presented (Figure 
75A, with cocaine) and the solution remained red within 2 hours because the formed CBSAcocaine complexes were highly resistant to the apurinic endonucleolytic activity of Exo III (Figure 75B, with cocaine). In contrast, the absorbance of AuNPs at $522 \mathrm{~nm}$ greatly decreased, and a broad absorbance peak appeared at $650 \mathrm{~nm}$ in the absence of cocaine (Figure 75A, without cocaine). We observed a clear red-to-blue color change with naked eye due to the aggregation of the AuNPs sheared by rapid Exo III digestion on the target-free CBSA assembly on AuNPs (Figure 75B, without cocaine).

\section{Effect of DNA surface coverage on Exo III- inhibited CBSA digestion}

DNA surface coverage can profoundly influence DNA hybridization efficiency, enzyme activity and assay sensitivity. ${ }^{164,166,167}$ We have proved that DTT or MCH-treated DNA-modified AuNPs can significantly increase DNA hybridization efficiency between surface-bound oligonucleotides and DNA target in solution. To obtain optimal surface coverage for cocaine detection described above, we first incubated different concentration of the DTT with the 5325XT-SF modified AuNPs for 30 minutes to yield different DNA surface coverage on AuNPs. The treated AuNPs were then mixed with the CBSA long fragment, cocaine and Exo III to perform CBSA digestion with or without cocaine.

We found that the DNA-modified AuNPs without any DTT treatment resulted in strong inhibition of Exo III activity, and the solution was in red regardless of the presence of cocaine. As the DTT concentration increased up to $200 \mu \mathrm{M}$, we observed the inhibitory effect of Exo III reaction with $250 \mu \mathrm{M}$ cocaine and a rapid Exo III digestion of cocaine-free CBSA assembly and a red-to-blue change in solution without cocaine. We believe that the six carbon ring formed by DTT displacement on the AuNP surface may provide favorable space to facilitate the typical 3way junction structured CBSA assembly. The digestion speed of Exo III greatly increased when we further increased the DTT concentration to reduce the DNA surface coverage (Figure 76), which is consistent with the results that we observed in the EATR-based DNA detection. 
However, the target-free CBSA complexes were also nonspecifically digested for the modified AuNPs with a lower surface coverage. We also noticed that DNA-modified AuNPs treated with $600 \mu \mathrm{M}$ DTT were not stable in our reaction buffer. Therefore, $500 \mu \mathrm{M}$ DTT was chosen for further optimization.
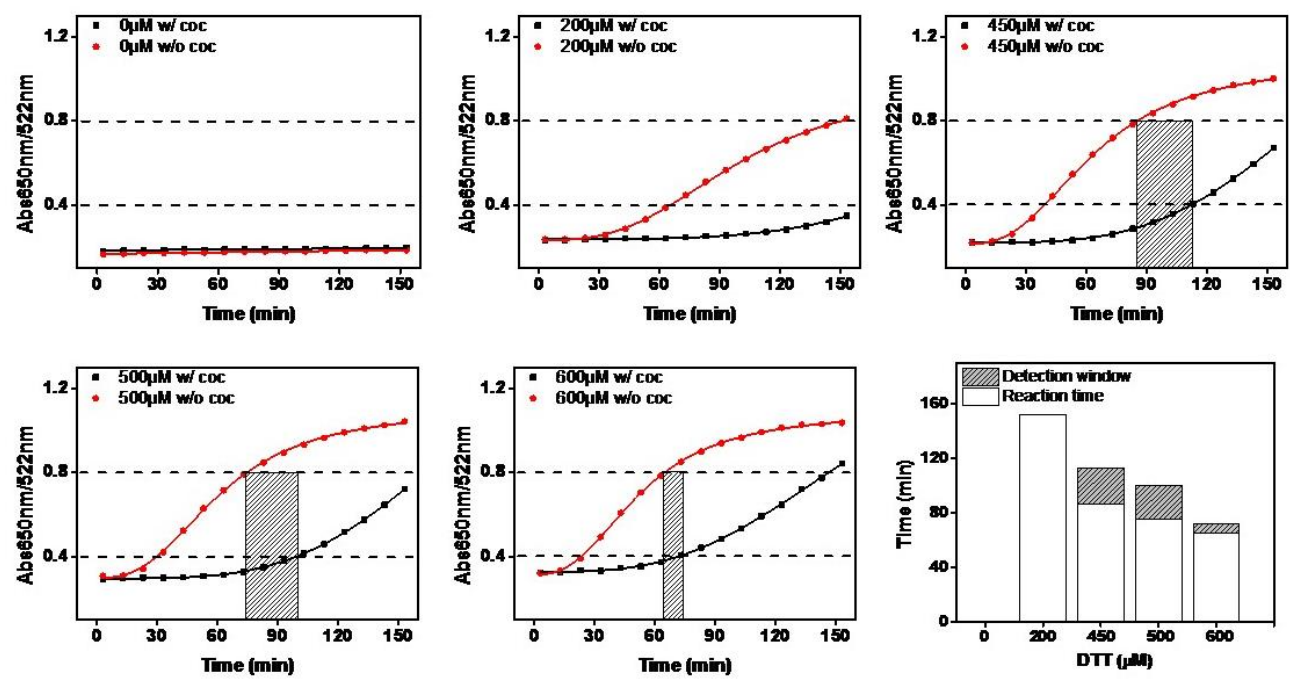

Figure 76. Effect of the DTT-regulated DNA surface coverage on Exo III activity for cocaine detection. The DNA modified AuNPs were first treated with different concentration of DTT (0 $600 \mu \mathrm{M})$ to obtain different surface coverage. The detection of cocaine were then performed in 25 $\mu \mathrm{L}$ of reaction buffer containing $10 \mathrm{mM}$ Tris buffer ( $\mathrm{pH} \mathrm{7.4),} 125 \mathrm{mM} \mathrm{NaCl}, 0.1 \mathrm{mM} \mathrm{MgCl}$, $1 \mu \mathrm{M}$ of $5 \mathrm{~A}-\mathrm{L}-5325$ long DNA fragment, and $10 \mathrm{U}$ Exo III, with or without cocaine $(250 \mu \mathrm{M})$ at room temperature.
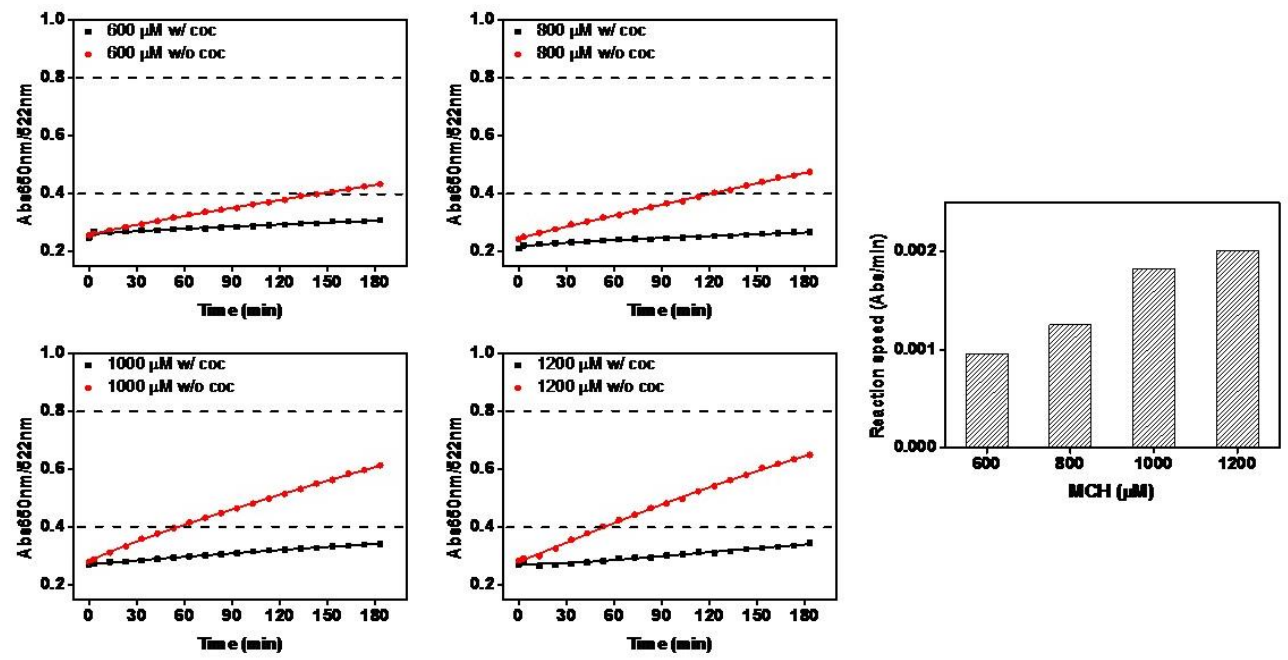

Figure 77. Effect of the MCH-regulated DNA surface coverage on Exo III activity for cocaine detection. The DNA modified AuNPs were first treated with different concentration of MCH (600 
- $1200 \mu \mathrm{M})$ to obtain different surface coverage. The detection of cocaine were then performed in $25 \mu \mathrm{L}$ of reaction buffer containing $10 \mathrm{mM}$ Tris buffer ( $\mathrm{pH} 7.4$ ), $125 \mathrm{mM} \mathrm{NaCl}, 0.1 \mathrm{mM} \mathrm{MgCl}_{2}$, $1 \mu \mathrm{M}$ of $5 \mathrm{~A}-\mathrm{L}-5325$ long DNA fragment, and $10 \mathrm{U}$ Exo III, with or without cocaine $(250 \mu \mathrm{M})$ at room temperature.

$\mathrm{MCH}$ was also used to reduce the DNA surface coverage on the AuNP as a surface diluent, and we have successfully proved MCH displacement on AuNP surface was favorable for DNA hybridization and enzyme-digestion resulted in aggregation of AuNPs in our EATR-based DNA detection assay. We observed that the reaction speed increased for Exo III-inhibited cocaine detection as the $\mathrm{MCH}$ concentration increased from $600 \mu \mathrm{M}$ to $1200 \mu \mathrm{M}$ (Figure 77). However, we could not observe a clear red-to-blue color change even after a 3-hour reaction. The $\mathrm{MCH}$ treated AuNPs gave a better colorimetric performance for DNA detection. We believe that the $\mathrm{MCH}$ displacement on the surface can significantly remove nonspecific adsorbed DNA and formed a mixed layer of $\mathrm{MCH}$ and DNA, which was favorable for DNA duplex formation. However, CBSA fragments assemble into two three-way junction domains, which are probably sensitive to steric effect and requires a large space to assemble CBSA fragments. We believe that MCH displacement is possible to affect CBSA assembly efficiency, Exo III activities or aggregation of AuNPs.
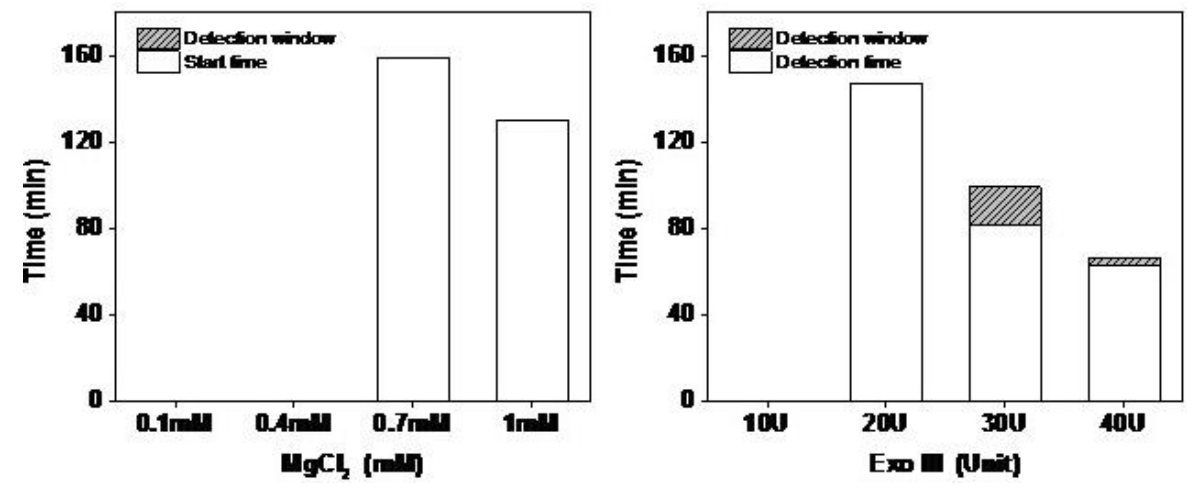

Figure 78. Effect of $\mathrm{MgCl}_{2}$ concentration and Exo III unit on the MCH-regulated DNA for cocaine detection. The DNA modified AuNPs were first treated with $1200 \mu \mathrm{M} \mathrm{MCH}$. The detection of cocaine were then performed in $25 \mu \mathrm{L}$ of reaction buffer contain $10 \mathrm{mM}$ Tris buffer $(\mathrm{pH} 7.4), 125 \mathrm{mM} \mathrm{NaCl}, 1 \mu \mathrm{M}$ of $5 \mathrm{~A}-\mathrm{L}-5325$ long DNA fragment, different concentration of $\mathrm{MgCl}_{2}$ or Exo III, with or without cocaine $(250 \mu \mathrm{M})$ at room temperature. 
We also increased the concentration of $\mathrm{MgCl}_{2}$ or Exo III to increase the reaction speed (Figure 78). Compared to DTT, the MCH displaced AuNP-DNA require a higher concentration of $\mathrm{MgCl}_{2}$ or Exo III to obtain the color change. However, both factors contributed to non-specific digestion. In conclusion, the reason that DTT displacement is favorable for the cocaine detection may be due to the six-carbon ring formed on the AuNP surface greatly reducing the steric effect.

\section{Test effect of the dynamic stability of complexes (3A to 6A long DNA) on Exo III-inhibited CBSA digestion}

The number of poly(A) linker on the $3^{\prime}$ of the long fragment played an important role in the dynamic stability of the cocaine-CBSA complex. The $5^{\prime}$ poly $\left(\mathrm{T}_{6}\right)$ acts as a flexible linker extending the active recognition portion of the oligonucleotide sequence away from the AuNP surface to improve target hybridization. ${ }^{70}$ Thus, polyA linker added to the $3^{\prime}$ of the long fragment promotes the hybridization between the CBSA fragments due to the hybridization of the poly $\mathrm{T}$ on the 5 ' of the short DNA. Here, we modified the long fragment with different lengths of polyA linker (named 3A to 6A L-5325). When L-5325 with 3A or 4A linker was used, the reaction was very slow, and the solution did not change to blue color even after 2.5 hours, and no clear difference observed in the presence and absence of cocaine solution (Figure 79). Clearly, the 3 or 4 AT base pairs were not strong enough to bring two fragments assembled and the Exo III could not effectively perform the AP incision. When we increased the linker to 5A, the binding of the short and long fragment were significantly improved. We observed that the solution changed to blue color in the absence of the cocaine due to an active endonuclease activity of Exo III incised on the AP site and the loss of the stability of the DNA-AuNP. Meanwhile, the cocaine-aptamer complex completely inhibited the enzyme activity, the solution remained in red. When we further increased the linker to 6A, we observed the inhibition of the Exo III's activity in the presence of cocaine. However, a 140-minute reaction was required in order to obtain a red-to-blue color change, which is 1.5 times longer reaction time than that obtained with the 5A linker (Figure 79). 

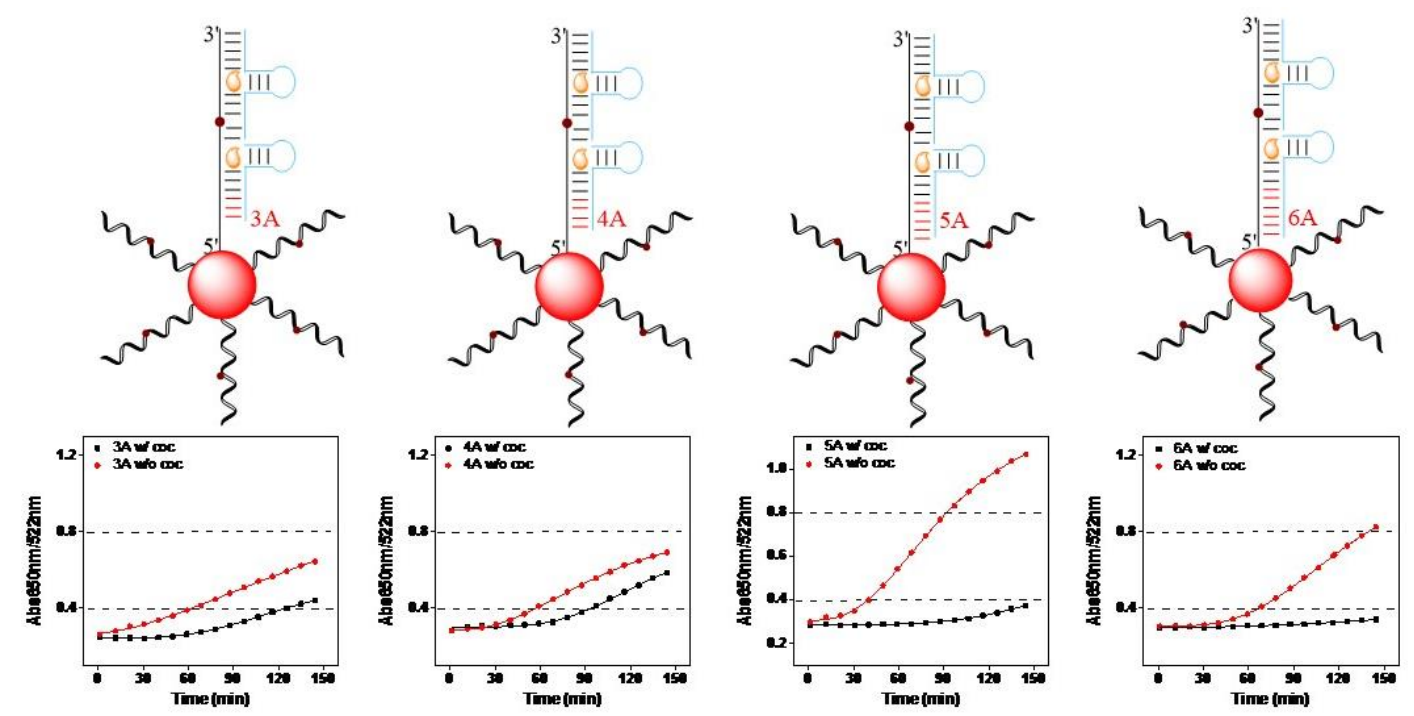

Figure 79. Effect of long CBSA aptamer fragment DNA on cocaine detection. The detection of cocaine were performed in $25 \mu \mathrm{L}$ of reaction buffer contains $10 \mathrm{mM}$ Tris buffer ( $\mathrm{pH} 7.4$ ) containing $125 \mathrm{mM} \mathrm{NaCl}, 0.1 \mathrm{mM} \mathrm{MgCl} 2,100 \mathrm{nM}$ short CBSA fragment-modified AuNPs, $1 \mu \mathrm{M}$ of the different long DNA fragment (named 3A to 6A-L-5325), and 10 U Exo III, with or without cocaine $(250 \mu \mathrm{M})$ at room temperature.

\section{Optimize NaCl concentration for Exo III-inhibited CBSA digestion}

Ionic strength is essential for the cocaine-induced split aptamer binding. We then optimized the $\mathrm{NaCl}$ concentration from $100 \mathrm{mM}$ to $200 \mathrm{mM}$ in the presence of $0.1 \mathrm{mM} \mathrm{MgCl}_{2}$. High concentration of $\mathrm{NaCl}$ is usually recommended for the hybridization since it shields the negative charge the DNA. When $100 \mathrm{mM} \mathrm{NaCl}$ was applied, the Exo III's digestion was inhibited in the presence of cocaine at the beginning. However, both samples changed from red to blue, indicating a small amount of duplex were formed in the presence of cocaine (Figure 80). When the $\mathrm{NaCl}$ concentration increased to $125 \mathrm{mM}$, we observed clear inhibition on the cocaine sample, while the sample without adding cocaine changed to blue color after 80 minutes (Figure 80). As the $\mathrm{NaCl}$ concentration increased, the sample with cocaine was in red, which indicated more cocaine-induced duplex was formed. When the $\mathrm{NaCl}$ concentration increased to $150 \mathrm{mM}$, we noticed 1.6 times slower reaction speed, which may cause by high concentration $\mathrm{NaCl}$ (Figure 
80). When the $\mathrm{NaCl}$ concentration increased to $200 \mathrm{mM}$, no color change in both samples were observed due to the total inhibition of Exo III activity. ${ }^{289}$ Therefore, $125 \mathrm{mM} \mathrm{NaCl}$ was used for further testing.
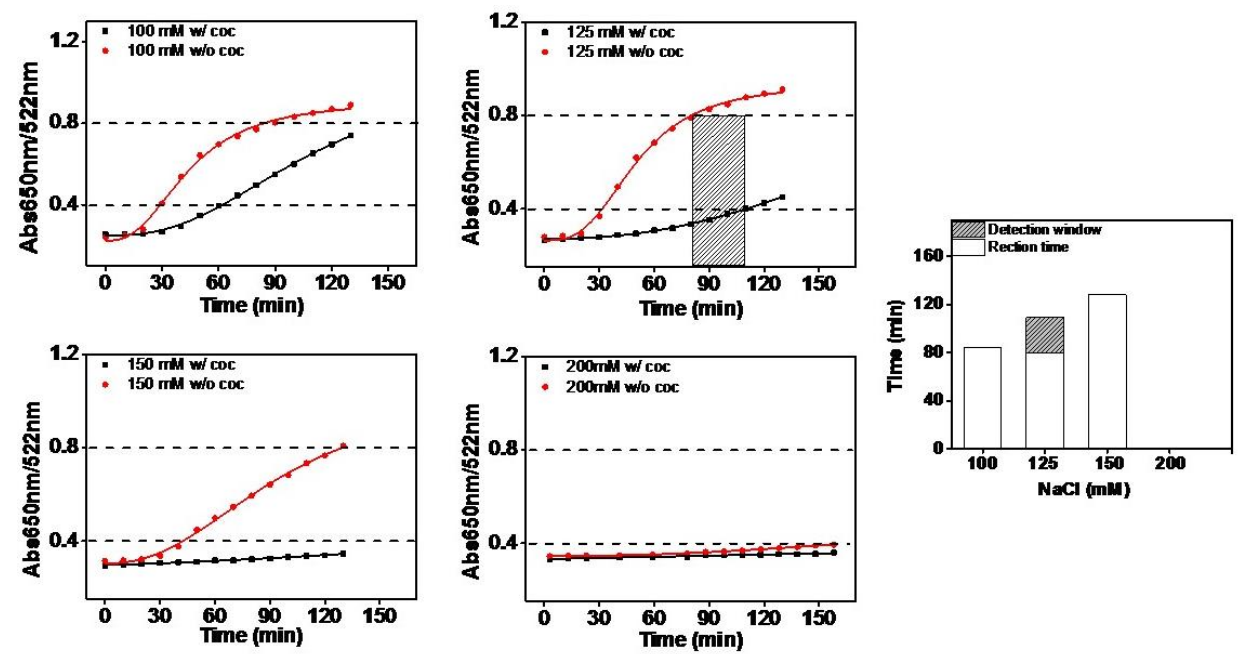

Figure 80. Effect of $\mathrm{NaCl}$ concentration on cocaine detection. The detection of cocaine were performed in $25 \mu \mathrm{L}$ of reaction buffer contains $10 \mathrm{mM}$ Tris buffer ( $\mathrm{pH}$ 7.4) containing $0.1 \mathrm{mM}$ $\mathrm{MgCl}_{2}, 100 \mathrm{nM}$ short CBSA fragment-modified AuNPs, $1 \mu \mathrm{M}$ of 5A-L-5325 DNA fragment, different concentration of $\mathrm{NaCl}$, and $10 \mathrm{U}$ Exo III, with or without cocaine $(250 \mu \mathrm{M})$ at room temperature.

\section{Optimize $\mathrm{MgCl}_{2}$ concentration for Exo III-inhibited CBSA digestion}

Exo III is an enzyme with AP endonuclease and exonuclease activities that are highly dependent on divalent cations. ${ }^{272}$ It is known that $\mathrm{Mg}^{2+}$ strongly stimulates the Exo III activity due to its proper ionic radius and electronegativity. Thus, the concentration of $\mathrm{Mg}^{2+}$ was varied from 0.1 to $1.0 \mathrm{mM}$ with a fixed concentration of $\mathrm{Na}^{+}(125 \mathrm{mM})$. It was found that the activity of Exo III increased with the increasing of $\mathrm{Mg}^{2+}$ concentration. We found that the AuNP color changed to blue after 30 minutes in the presence of $1.0 \mathrm{mM} \mathrm{Mg}^{2+}$, however, the detection window was only 2 minutes; a maximum detection window of 25 minutes was achieved in the presence of $0.1 \mathrm{mM}$ $\mathrm{Mg}^{2+}$ after 90 minutes (Figure 81 ). When the $\mathrm{Mg}^{2+}$ concentration decreased to $0.05 \mathrm{mM}, 150$ minutes was required to obtain the color change. In order to achieve the faster reaction and better detection window, $0.1 \mathrm{mM} \mathrm{Mg}{ }^{2+}$ was used in the following experiments. 

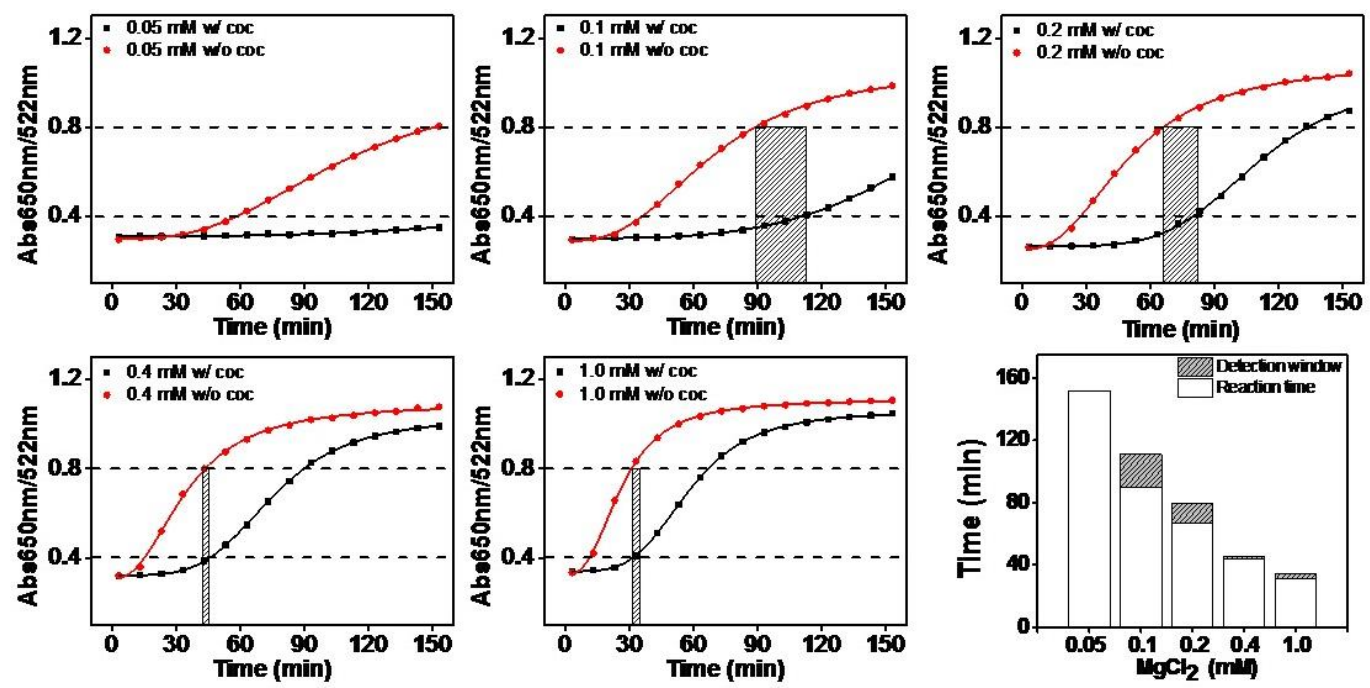

Figure 81. Effect of $\mathrm{MgCl}_{2}$ concentration on the cocaine detection. The detection of cocaine were performed in $25 \mu \mathrm{L}$ of reaction buffer contained $10 \mathrm{mM}$ Tris buffer ( $\mathrm{pH}$ 7.4) containing $125 \mathrm{mM}$ $\mathrm{NaCl}, 100 \mathrm{nM}$ short CBSA fragment-modified AuNPs, $1 \mu \mathrm{M}$ of 5A-L-5325 DNA fragment, different concentration of $\mathrm{MgCl}_{2}$, and $10 \mathrm{U}$ Exo III, with or without cocaine $(250 \mu \mathrm{M})$ at room temperature.

\section{Optimize long CBSA fragment concentration for Exo III-inhibited CBSA digestion}

The target-induced duplex formed between short DNA modified on the AuNP and long fragment in the presence of cocaine. Thus, the concentration of the long fragment may play an important role in the cocaine detection. To test the inhibitory effect of cocaine on the Exo III digestion, we varied the long fragment concentration from $0.1 \mu \mathrm{M}$ to $8 \mu \mathrm{M}$ (Figure 82). As the long fragment concentration increased from $0.1 \mu \mathrm{M}$ to $1 \mu \mathrm{M}$, we noticed increased inhibitory effect on the Exo III's digestion, which meant the hybridization efficiency increased as the concentration of long DNA increased. When we further increased the long fragment to $2 \mu \mathrm{M}$, we noticed a long detection window and a slower reaction (Figure 82). When the long fragment further was increased to $4 \mu \mathrm{M}$, we only observed the clear color change after 4 hours. No color change was observed when the long fragment concentration increased to $8 \mu \mathrm{M}$, due to the excess amount of free DNA stabilize the AuNP from aggregation, which was consistent with the literature report. ${ }^{64}$ 

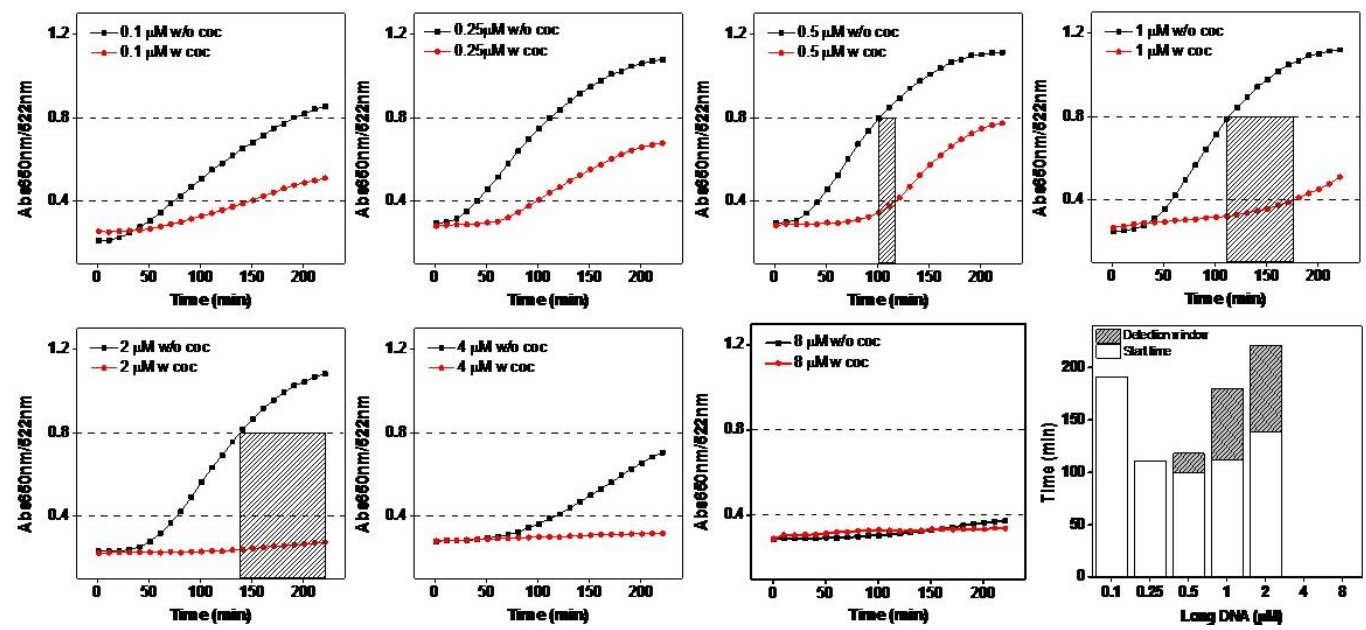

Figure 82. Effect of long CBSA fragment concentration on cocaine detection. The detection of cocaine were performed in $25 \mu \mathrm{L}$ of reaction buffer contained $10 \mathrm{mM}$ Tris buffer ( $\mathrm{pH} 7.4$ ) containing $125 \mathrm{mM} \mathrm{NaCl}, 0.1 \mathrm{mM} \mathrm{MgCl} 2,100 \mathrm{nM}$ short CBSA fragment-modified AuNPs, different concentration of 5A-L-5325 DNA fragment and 10 U Exo III, with or without cocaine $(250 \mu \mathrm{M})$ at room temperature.

\section{Optimize Exo III concentration for Exo III-inhibited CBSA digestion}
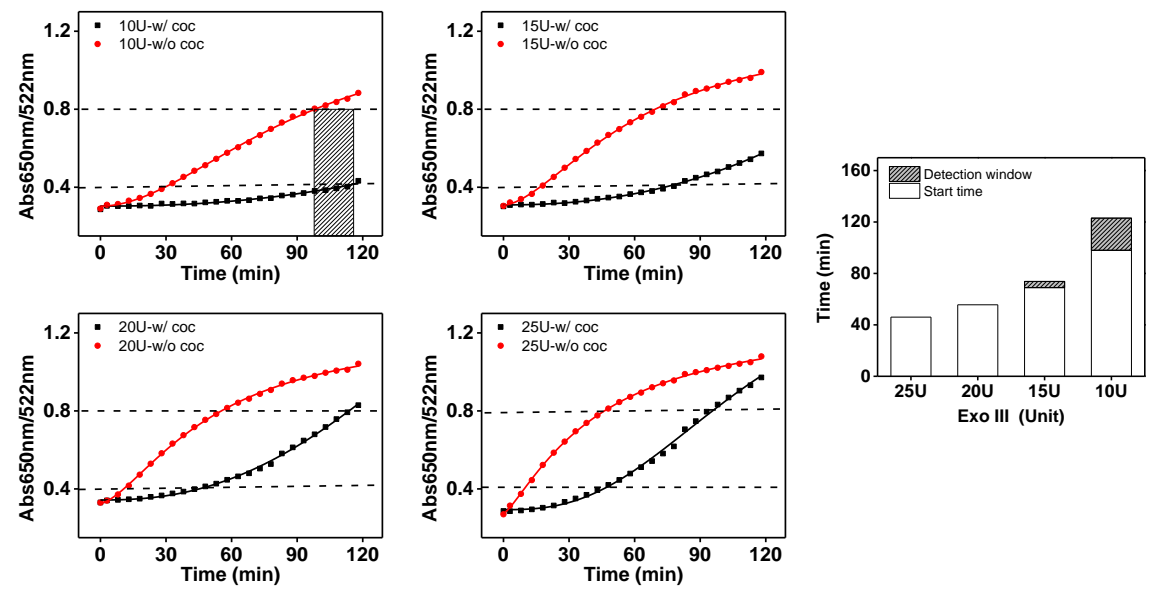

Figure 83. Effect of Exo III concentration effect on the cocaine detection. The detection of cocaine were performed in $25 \mu \mathrm{L}$ of reaction buffer contained $10 \mathrm{mM}$ Tris buffer $(\mathrm{pH} 7.4)$ containing $125 \mathrm{mM} \mathrm{NaCl}, 0.1 \mathrm{mM} \mathrm{MgCl} 2,100 \mathrm{nM}$ short CBSA fragment-modified AuNPs, $1 \mu \mathrm{M}$ 5A-L-5325 DNA fragment and different concentration of Exo III, with or without cocaine (250 $\mu \mathrm{M})$ at room temperature.

In order to apply our colorimetric detection of cocaine for fast field test, we need to increase the reaction speed. The easiest way is to simply increase the enzyme concentration. It was found 
that the reaction time decreased to 46 minutes along with the increasing of Exo III from $10 \mathrm{U}$ to $25 \mathrm{U}$ (Figure 83), which was only half of the detection time when we applied $10 \mathrm{U}$. However, we noticed that the nonspecific digestion increased as the Exo III concentration increased, which significantly decreased the detection window.

\section{Cocaine concentration dependence on Exo III-inhibited CBSA digestion}

Having demonstrated proof-of-concept, we further tested the sensitivity of our Exo III-based assay by generating a calibration curve using different concentrations of cocaine. The concentration of cocaine can be determined by monitoring with the naked eye or a UV-Vis spectrometer at which the blue-to-red color change associated aggregation occurred. The signal decreased proportionally to cocaine concentration with a nonlinear range (Figure 84). Our detection limit is $1 \mu \mathrm{M}$, which is comparable with other colorimetric sensors.

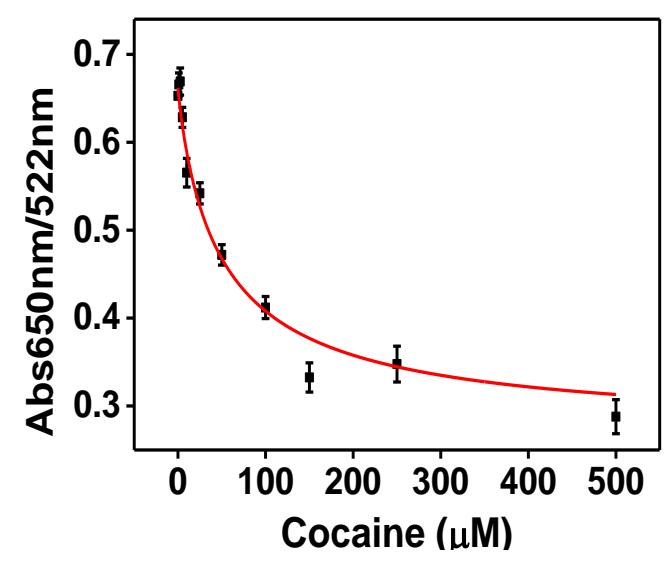

Figure 84. Calibration curve of colorimetric detection of cocaine under optimized experimental conduction.

In order to achieve fast reaction and better selectivity, we also engineered other CBSA aptamers by adding or deleting the base pair above and below the abasic site (Figure 85), and we characterized the digestion product in the presence of Exo III. When challenged with same concentration of Exo III, we did not observe the digestion product for CBSA 5225; for CBSA 5425, we did observe the Exo III inhibition in the presence of cocaine; while for CBSA 5335, all 
of the short fragments were digested, only 14-nt band observed, which may be due to the fast reaction. By comparing the result, we found the base pair significantly improve the reaction speed and selectivity. Next, we will keep working on selecting the best DNA sequences to achieve highest reaction speed.

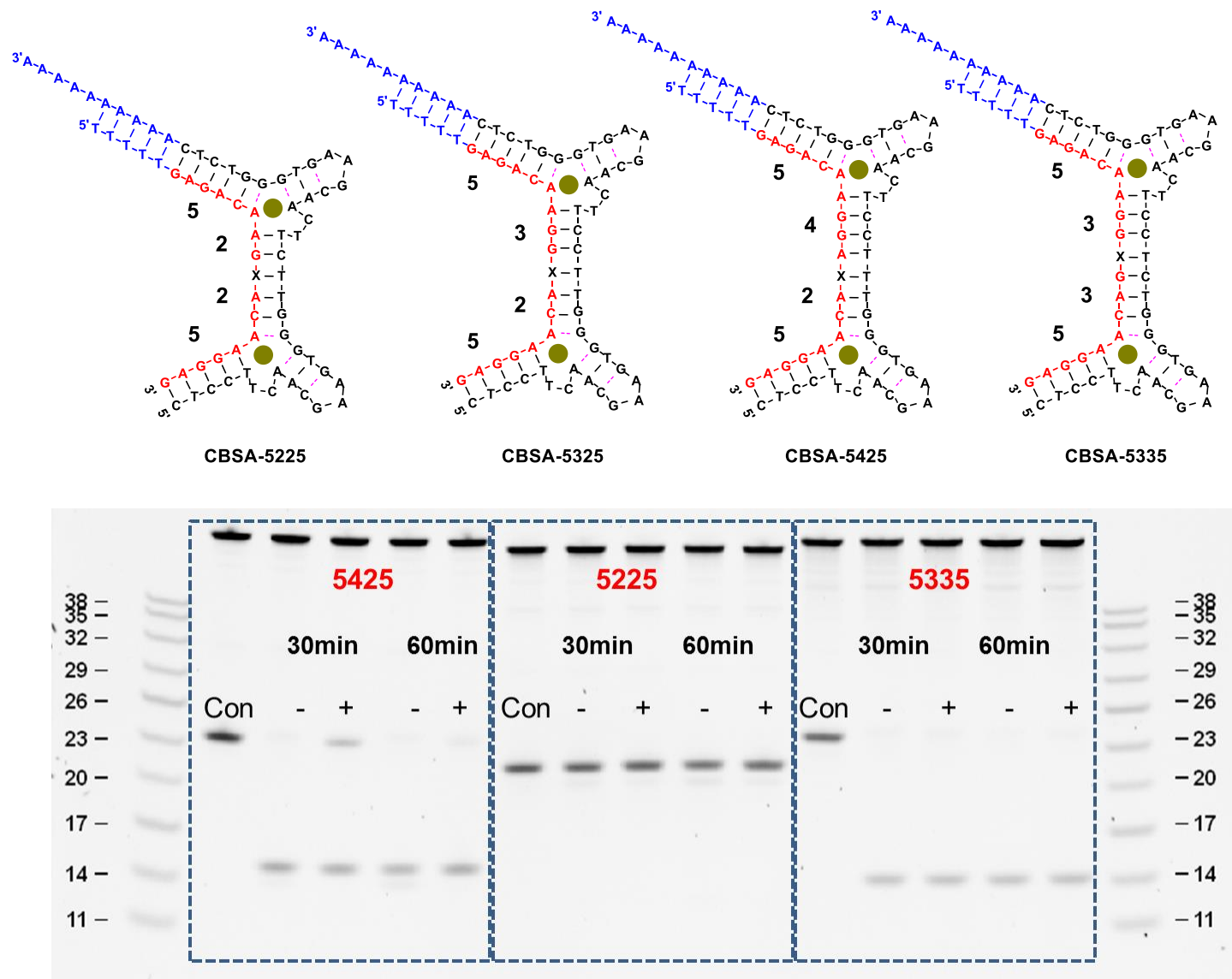

Figure 85. Engineering new CBSA aptamers to achieve a fast reaction with a better selectivity.

\subsection{Conclusion}

To conclude this chapter, we first compared the accuracy of three different assays for characterization of DNA surface coverage on AuNPs for fluorophore dye labeled DNA and nonlabeled DNA. We found the OliGreen based solution assay allowed an accurate determination of non-labeled DNA surface coverage on AuNPs. Second, we developed a simple and rapid assay to regulate the surface coverage after one-pot synthesis of DNA-modified AuNP by backfilling the 
oligonucleotides immobilized on the AuNP surface with small thiolated molecules such as MCH and DTT. We then test the surface coverage effect on the assay performance for Exo III-assisted, target-recycled DNA detection; we found the surface coverage plays a significant role in DNA hybridization and enzyme reaction. With our methods, only single AuNP modification needs to be done, the surface coverage of the DNA can be easily controlled by varying the diluent concentrations. Third, we proved the effect of DNA surface coverage on Exo III-assisted small molecule detection. Using cocaine as the model, we engineered single binding pocket split aptamer to dual binding pockets split aptamer and tested the Exo III digestion product, and we found the selectivity is significantly improved by incorporating an abasic site. Finally, we modified the short fragment onto AuNP surface, and developed an AuNP-reported colorimetric detection of cocaine based on Exo III inhibition on the target-aptamer complex. In the future, we will focus on increase the reaction speed and lower the detection limit in complex samples. 


\section{CHAPTER 6: Summary and future work}

\subsection{Summary}

This dissertation describes the application of AuNPs as a colorimetric probe based on their distinctive size- and shape-dependent optical properties. The focus of the study is to develop highly sensitive colorimetric platforms for small-molecule and DNA detection based on the following strategies: i) using unmodified AuNPs as a chemical sensor for colorimetrically detecting the dehydrogenase cofactor NADH in an instrument-free manner; ii) using an EATRbased strategy to achieve amplified detection of SNPs with DNA-modified AuNPs; and iii) increasing the sensitivity and speed of AuNP-mediated detection by adjusting DNA surface coverage on the AuNPs with small thiolated molecules, and demonstrating the effects of surface coverage on DNA and small-molecule detection.

AuNPs have been directly used for colorimetric detection based on the biocatalytic growth process. However, the size of the AuNPs can also be reduced via chemically disintegration through the effects of oxidative species and complexation ligands, and several colorimetric sensors have been based on this principle. However, only inorganic targets have been detected to date, with sophisticated instruments such as UV-Vis spectrometers required to obtain the signal. Accordingly, such assays are not suitable for point-of-care diagnostics or on-site measurement. One focus of this study was to develop instrument-free detection for biologically important molecules. We therefore developed a chemical sensor for NADH detection, based on AuNPcoated film on a paper substrate. This device achieved rapid, simple and efficient detection of $\mathrm{NADH}$ in sample volumes as small as $25 \mu \mathrm{L}$ within 4 minutes at room temperature, with a very low detection limit $(12.5 \mu \mathrm{M})$. We further demonstrated that this paper-based device can also be applied for detecting NADH produced by GDH, and for screening for inhibitors of this enzyme.

AuNPs can be also functionalized with biomolecules such as protein or DNA through a covalent bond. For the second component of this study, we demonstrated a colorimetric SNP 
detection assay that can be performed in a single tube with only one set of DNA probe-modified AuNPs, Exo III enzyme, and target DNA. Single-base mismatch detection is achieved through the dual activities of Exo III. In the presence of a perfectly matched DNA target, Exo III can recognize and cleave the abasic sites within the probe, and subsequently digests the nicked duplexes by its exonucleolytic activity. The target strand is subsequently released, hybridizing to a new probe for a new cycle of digestion, until complete removal of probe sequences results in a visible red-to-blue color change due to AuNP aggregation in the high ionic strength environment. This target-recycling also leads to improved sensitivity. In contrast, SNP-containing, mismatched targets inhibit endonucleolytic activity and therefore stall exonucleolytic digestion, so that the AuNP solution remains red in color. In our assay, AuNP-DNA conjugates serve as a sensitive signal reporter due to their extremely large molar extinction coefficient and excellent sequence specificity, directly reporting the presence of SNPs based on a red-to-blue color change without the need for any additional instruments.

DNA surface coverage plays an important role in sensor performance. In the third part of the dissertation, we first validated a method for characterizing the surface density of unlabeled DNA on AuNPs. We then adjusted the surface coverage on the AuNPs using thiolated molecules DTT and $\mathrm{MCH}$, and tested the effects of DNA surface coverage on DNA detection using the colorimetric detection platform discussed in Chapter 4. We found that DNA surface coverage strongly affects the reaction time required to achieve a clear red-to-blue change in our EATRbased colorimetric assay. In order to demonstrate the effects of surface coverage on smallmolecule detection, we engineered our cocaine aptamer into a split aptamer. After conjugating this split aptamer onto AuNPs, we proved that surface coverage also plays an important role in small molecule detection. 


\subsection{Future work}

This dissertation demonstrates the application of AuNPs for the instrument-free, rapid, colorimetric detection of small molecules and DNA. The work specifically focused on the application of unmodified AuNPs for NADH detection, and tested GDH and its inhibitors as a model system. In the future, our paper-based device could offer a valuable and low-cost analytical tool for monitoring $\mathrm{NAD}^{+}$-associated enzymatic reactions and screening for dehydrogenase inhibitors in a variety of testing contexts. DNA functionalized AuNPs, another well-studied nanoconjugate, have been demonstrated as an effective tool for detecting both DNA SNPs and cocaine. In future studies, we believe our DTT displacement method can work as a general method to obtain the optimal surface coverage for the detection of any other target molecules. Our colorimetric detection platform can be easily transferred into a multiplexed, paper-based device to permit rapid, cheap and immediate screening of SNPs and a variety of non-DNA targets, including proteins, small molecules, metal ions and even whole cells. 


\section{References}

1. Thevenot, D. R.; Toth, K.; Durst, R.; Wilson, G. S. Electrochemical Biosensors: Recommended Definitions and Classification. Pure Appl. Chem. 1999, 71 (12), 2333 - 2348.

2. Monošík, R.; Stred’anský, M.; Šturdík, E. Biosensors - Classification, Characterization and New Trends. Acta Chimica Slovaca, 2012, 5 (1) 109-120.

3. Rao, C. N. R.; Kulkarni, G. U.; Thomas, P. J.; Edwards, P. P. Size-Dependent Chemistry: Properties of Nanocrystals. Chem. Eur. J. 2002, 8 (1), 28-35.

4. Kelly, K. L.; Coronado, E.; Zhao, L. L.; Schatz, G. C. The Optical Properties of Metal Nanoparticles: the Influence of Size, Shape, and Dielectric Environment. J. Phys. Chem. B 2002, 107 (3), 668-677.

5. Elghanian, R.; Storhoff, J. J.; Mucic, R. C.; Letsinger, R. L.; Mirkin, C. A. Selective Colorimetric Detection of Polynucleotides Based on the Distance-Dependent Optical Properties of Gold Nanoparticles. Science 1997, 277 (5329), 1078-1081.

6. Valden, M.; Lai, X.; Goodman, D. W. Onset of Catalytic Activity of Gold Clusters on Titania with the Appearance of Nonmetallic Properties. Science 1998, 281 (5383), 1647-1650.

7. Faraday, M. The Bakerian Lecture: Experimental Relations of Gold (and Other Metals) to Light. Phil. Trans. R. Soc. Lond. 1857, 147, 145-181.

8. Turkevich, J.; Stevenson, P. C.; Hillier, J. A Study of the Nucleation and Growth Processes in the Synthesis of Colloidal Gold. Discuss. Faraday Soc. 1951, 11, 55-75.

9. Brust, M.; Walker, M.; Bethell, D.; Schiffrin, D. J.; Whyman, R. Synthesis of Thiolderivatised Gold Nanoparticles in a Two-phase Liquid-Liquid System. J. Chem. Soc., Chem. Commun. 1994, (7), 801-802.

10. Busbee, B. D.; Obare, S. O.; Murphy, C. J. An Improved Synthesis of High-Aspect-Ratio Gold Nanorods. Adv. Mater. 2003, 15 (5), 414-416.

11. Senthil Kumar, P.; Pastoriza-Santos, I.; Rodríguez-González, B.; Javier García de Abajo, F.; Liz-Marzán, L. M. High-Yield Synthesis and Optical Response of Gold Nanostars. Nanotechnology 2008, 19 (1), 015606.

12. Millstone, J. E.; Park, S.; Shuford, K. L.; Qin, L.; Schatz, G. C.; Mirkin, C. A. Observation of a Quadrupole Plasmon Mode for a Colloidal Solution of Gold Nanoprisms. J. Am. Chem. Soc. 2005, 127 (15), 5312-5313.

13. Kim, F.; Connor, S.; Song, H.; Kuykendall, T.; Yang, P. D. Platonic Gold Nanocrystals. Angew. Chem. Int. Ed. 2004, 43 (28), 3673-3677.

14. Aguirre, C. M.; Kaspar, T. R.; Radloff, C.; Halas, N. J. CTAB Mediated Reshaping of Metallodielectric Nanoparticles. Nano Lett. 2003, 3 (12), 1707-1711. 
15. Nikoobakht, B.; El-Sayed, M. A. Preparation and Growth Mechanism of Gold Nanorods (NRs) Using Seed-Mediated Growth Method. Chem. Mater. 2003, 15 (10), 1957-1962.

16. Mie, G. Beiträge zur Optik trüber Medien, speziell kolloidaler Metallösungen. Ann. Phys. (Berlin) 1908, 330 (3), 377-445.

17. Willets, K. A.; Van Duyne, R. P. Localized Surface Plasmon Resonance Spectroscopy and Sensing. Annu Rev Phys Chem. 2007, 58, 267-297.

18. Liz-Marzán, L. M. Tailoring Surface Plasmons through the Morphology and Assembly of Metal Nanoparticles. Langmuir 2006, 22 (1), 32-41.

19. Li, M.; Cushing S. K.; Zhang J. M.; Lankford J.; Aguilar Z. P.; Ma D. L.; Wu N. Q. ShapeDependent Surface-Enhanced Raman Scattering in Gold-Raman-Probe-Silica Sandwiched Nanoparticles for Biocompatible Applications. Nanotechnology 2012, 23 (11), 115501.

20. Zhang, X. F.; Zhao, H.; Xue, Y.; Wu, Z. J.; Zhang, Y. J.; He, Y.; Li, X. J.; Yuan, Z. B. Colorimetric Sensing of Clenbuterol Using Gold Nanoparticles in the Presence of Melamine. Biosens. Bioelectron. 2012, 34 (1), 112-117.

21. Xu, H.; Wang, Y. W.; Huang, X. M.; Li, Y.; Zhang, H.; Zhong, X. H. $\mathrm{Hg}^{2+}-$ Mediated Aggregation of Gold Nanoparticles for Colorimetric Screening of Biothiols. Analyst 2012, 137 (4), 924-931.

22. Xiao, Y.; Pavlov, V.; Levine, S.; Niazov, T.; Markovitch, G.; Willner, I. Catalytic Growth of $\mathrm{Au}$ Nanoparticles by NAD(P)H Cofactors: Optical Sensors for NAD $(\mathrm{P})^{+}$-Dependent Biocatalyzed Transformations. Angew. Chem. 2004, 116 (34), 4619-4622.

23. Jena, B. K.; Raj, C. R. Electrochemical Biosensor Based on Integrated Assembly of Dehydrogenase Enzymes and Gold Nanoparticles. Anal. Chem. 2006, 78 (18), 6332-6339.

24. Tang, L.; Zeng, G. M.; Shen, G. L.; Zhang, Y. P; Li, Y.; Fan, C. Z.; Liu, C.; Niu, C. G. Highly Sensitive Sensor for Detection of NADH Based on Catalytic Growth of Au Nanoparticles on Glassy Carbon Electrode. Anal. Bioanal. Chem. 2009, 393 (6), 1677-1684.

25. Jana, N. R.; Gearheart, L.; Murphy, C. J. Seeding Growth for Size Control of 5-40 nm Diameter Gold Nanoparticles. Langmuir 2001, 17 (22), 6782-6786.

26. Liu, Q.; Jing, C.; Zheng, X. X.; Gu, Z.; Li, D.; Li, D. W.; Huang, Q.; Long, Y. T.; Fan, C. H. Nanoplasmonic Detection of Adenosine Triphosphate by Aptamer Regulated Self-Catalytic Growth of Single Gold Nanoparticles. Chem. Commun. 2012, 48 (77), 9574-9576.

27. Wang, L. H.; Liu, X. F.; Hu, X. F.; Song, S. P.; Fan, C. H. Unmodified Gold Nanoparticles as a Colorimetric Probe for Potassium DNA Aptamers. Chem. Commun. 2006, 42 (36), 37803782 .

28. Xia, F.; Zuo, X.; Yang, R.; Xiao, Y.; Kang, D.; Vallée-Bélisle, A.; Gong, X.; Yuen, J. D.; Hsu, B. B. Y.; Heeger, A. J.; Plaxco, K. W. Colorimetric Detection of DNA, Small Molecules, Proteins, and Ions Using Unmodified Gold Nanoparticles and Conjugated Polyelectrolytes. Proc. Natl. Acad. Sci. USA 2010, 107 (24), 10837-10841. 
29. Liu, M. Y.; Yuan, M.; Lou, X. H.; Mao, H. J.; Zheng, D. M.; Zou, R. X.; Zou, N. L.; Tang, X. R.; Zhao, J. L. Label-Free Optical Detection of Single-Base Mismatches by the Combination of Nuclease and Gold Nanoparticles. Biosens. Bioelectron. 2011, 26 (11), 4294-4300.

30. Zhang, M.; Liu, Y. Q.; Ye, B. C. Colorimetric Assay for Sulfate Using Positively-Charged Gold Nanoparticles and its Application for Real-Time Monitoring of Redox Process. Analyst 2011, 136 (21), 4558-4562.

31. Liu, J. W.; Lu, Y. A Colorimetric Lead Biosensor Using DNAzyme-Directed Assembly of Gold Nanoparticles. J. Am. Chem. Soc. 2003, 125 (22), 6642-6643.

32. Lou, T.; Chen, L.; Zhang, C. R.; Kang, Q.; You, H. Y.; Shen, D. Z.; Chen, L. X. A Simple and Sensitive Colorimetric Method for Detection of Mercury Ions Based on AntiAggregation of Gold Nanoparticles. Anal. Methods 2012, 4 (2), 488-491.

33. Ma, Z. F.; Sui, S. F. Naked-Eye Sensitive Detection of Immunoglubulin G by Enlargement of Au Nanoparticles In Vitro. Angew. Chem. Int. Ed. 2002, 41 (12), 2176-2179.

34. Zayats, M.; Baron, R.; Popov, I.; Willner, I. Biocatalytic Growth of Au Nanoparticles: From Mechanistic Aspects to Biosensors Design. Nano Letters 2005, 5 (1), 21-25.

35. Wang, J.; Zhou, N. D.; Zhu, Z. Q.; Huang, J. Y.; Li, G. X. Detection of Flavonoids and Assay for Their Antioxidant Activity Based on Enlargement of Gold Nanoparticles. Anal. Bioanal. Chem. 2007, 388 (5-6), 1199-1205.

36. Liu, X.; Xu, H.; Xia, H.; Wang, D. Rapid Seeded Growth of Monodisperse, Quasi-Spherical, Citrate-Stabilized Gold Nanoparticles via $\mathrm{H}_{2} \mathrm{O}_{2}$ Reduction. Langmuir 2012, 28 (38), 1372013726.

37. Baron, R.; Zayats, M.; Willner, I. Dopamine-, L-DOPA-, Adrenaline-, and NoradrenalineInduced Growth of Au Nanoparticles: Assays for the Detection of Neurotransmitters and of Tyrosinase Activity. Anal. Chem. 2005, 77 (6), 1566-1571.

38. Pavlov, V.; Xiao, Y.; Shlyahovsky, B.; Willner, I. Aptamer-Functionalized Au Nanoparticles for the Amplified Optical Detection of Thrombin. J. Am. Chem. Soc. 2004, 126 (38), 11768 11769.

39. Pavlov, V.; Xiao, Y.; Willner, I. Inhibition of the Acetycholine Esterase-Stimulated Growth of Au Nanoparticles: Nanotechnology-Based Sensing of Nerve Gases. Nano Lett. 2005, 5 (4), 649-653.

40. Shang, L.; Chen, H. J.; Deng, L.; Dong, S. J. Enhanced Resonance Light Scattering Based on Biocatalytic Growth of Gold Nanoparticles for Biosensors Design. Biosens. Bioelectron. 2008, 23 (7), 1180-1184.

41. Lim, S. Y.; Kim, J. H.; Lee, J. S.; Park, C. B. Gold Nanoparticle Enlargement Coupled with Fluorescence Quenching for Highly Sensitive Detection of Analytes. Langmuir 2009, 25 (23), 13302-13305. 
42. Zhou, N. D.; Wang, J.; Chen, T.; Yu, Z. G.; Li, G. X. Enlargement of Gold Nanoparticles on the Surface of a Self-Assembled Monolayer Modified Electrode: A Mode in Biosensor Design. Anal. Chem. 2006, 78 (14), 5227-5230.

43. Weisbecker, C. S.; Merritt, M. V.; Whitesides, G. M. Molecular Self-Assembly of Aliphatic Thiols on Gold Colloids. Langmuir 1996, 12 (16), 3763-3772.

44. McCarthy, A. J.; Coleman, R. G.; Nicol, M. J. The Mechanism of the Oxidative Dissolution of Colloidal Gold in Cyanide Media. J. Electrochem. Soc. 1998, 145 (2), 408-414.

45. Lou, X. D.; Zhang, Y.; Qin, J. Q.; Li, Z. A Highly Sensitive and Selective Fluorescent Probe for Cyanide Based on the Dissolution of Gold Nanoparticles and its Application in Real Samples. Chem. Eur. J. 2011, 17 (35), 9691-9696.

46. Liu, C. Y.; Tseng, W. L. Colorimetric Assay for Cyanide and Cyanogenic Glycoside Using Polysorbate 40-Stabilized Gold Nanoparticles. Chem. Commun. 2011, 47 (9), 2550-2552.

47. Pal, T.; Jana, N. R.; Sau, T. K. Nucleophile Induced Dissolution of Gold. Corros. Sci. 1997, 39 (5), 981-986.

48. Tripathy, S. K.; Woo, J. Y.; Han, C. S. Highly Selective Colorimetric Detection of Hydrochloric Acid Using Unlabeled Gold Nanoparticles and an Oxidizing Agent. Anal. Chem. 2011, 83 (24), 9206-9212.

49. Praharaj, S.; Panigrahi, S.; Basu, S.; Pande, S.; Jana, S.; Ghosh, S. K.; Pal, T. Effect of Bromide and Chloride Ions for the Dissolution of Colloidal Gold. J. Photochem. Photobiol. A: Chem. 2007, 187 (2-3), 196-201.

50. Sreeprasad, T. S.; Samal, A. K.; Pradeep, T. Body- or Tip-Controlled Reactivity of Gold Nanorods and Their Conversion to Particles through Other Anisotropic Structures. Langmuir 2007, 23 (18), 9463-9471.

51. Chen, Y. Y.; Chang, H. T.; Shiang, Y. C.; Hung, Y. L.; Chiang, C. K.; Huang, C. C. Colorimetric Assay for Lead Ions Based on the Leaching of Gold Nanoparticles. Anal. Chem. 2009, 81 (22), 9433-9439.

52. Dasog, M.; Scott, R. W. J. Understanding the Oxidative Stability of Gold MonolayerProtected Clusters in the Presence of Halide Ions under Ambient Conditions. Langmuir 2007, 23 (6), 3381-3387.

53. Rodríguez-Fernández, J.; Pérez-Juste, J.; Mulvaney, P.; Liz-Marzán, L. M. Spatially-Directed Oxidation of Gold Nanoparticles by Au(III)-CTAB Complexes. J. Phys. Chem. B 2005, 109 (30), 14257-14261.

54. Goulet, P. J. G.; Leonardi, A.; Lennox, R. B. Oxidation of Gold Nanoparticles by Au(III) Complexes in Toluene. J. Phys. Chem. C 2012, 116 (26), 14096-14102.

55. Khanal, B. P.; Zubarev, E. R. Purification of High Aspect Ratio Gold Nanorods: Complete Removal of Platelets. J. Am. Chem. Soc. 2008, 130 (38), 12634-12635. 
56. Zou, R. X.; Guo, X.; Yang, J.; Li, D. D.; Peng, F.; Zhang, L.; Wang, H. J.; Yu, H. Selective Etching of Gold Nanorods by Ferric Chloride at Room Temperature. CrystEngComm 2009, 11 (12), 2797-2803.

57. Zhang, Z. Y.; Chen, Z. P.; Qu, C. L.; Chen, L. X. Highly Sensitive Visual Detection of Copper Ions Based on the Shape-Dependent LSPR Spectroscopy of Gold Nanorods. Langmuir 2014, 30 (12), 3625-3630.

58. Liu, R. L.; Chen, Z. P.; Wang, S. S.; Qu, C. L.; Chen, L. X.; Wang, Z. Colorimetric Sensing of Copper(II) Based on Catalytic Etching of Gold Nanoparticles. Talanta 2013, 112, 37-42.

59.Li, F. M.; Liu, J. M.; Wang, X. X.; Lin, L. P.; Cai, W. L.; Lin, X.; Zeng, Y. N.; Li, Z. M.; Lin, S. Q. Non-aggregation Based Label Free Colorimetric Sensor for the Detection of Cr (VI) Based on Selective Etching of Gold Nanorods. Sensors Actuators B: Chem. 2011, 155 (2), 817-822.

60. Chandrasekar, G.; Mougin, K.; Haidara, H.; Vidal, L.; Gnecco, E. Shape and Size Transformation of Gold Nanorods (GNRs) via Oxidation Process: A Reverse Growth Mechanism. Appl. Surf. Sci. 2011, 257 (9), 4175-4179.

61. Ni, W. H.; Kou, X. S.; Yang, Z.; Wang, J. F. Tailoring Longitudinal Surface Plasmon Wavelengths, Scattering and Absorption Cross Sections of Gold Nanorods. ACS Nano 2008, 2 (4), 677-686.

62. Chen, Z. P.; Zhang, Z. Y.; Qu, C. L.; Pan, D. W.; Chen, L. X. Highly Sensitive Label-Free Colorimetric Sensing of Nitrite Based on Etching of Gold Nanorods. Analyst 2012, 137 (22), 5197-5200.

63. Liu, J. M.; Jiao, L.; Cui, M. L.; Lin, L. P.; Wang, X. X.; Zheng, Z. Y.; Zhang, L. H.; Jiang, S. L. A Highly Sensitive Non-aggregation Colorimetric Sensor for the Determination of I Based on its Catalytic Effect on $\mathrm{Fe}^{3+}$ Etching Gold Nanorods. Sensors Actuators B: Chem. 2013, 188, 644-650.

64. Li, H. X.; Rothberg, L. Colorimetric Detection of DNA Sequences Based on Electrostatic Interactions With Unmodified Gold Nanoparticles. Proc. Natl. Acad. Sci. U. S. A. 2004, 101 (39), 14036-14039.

65. Lesniewski, A.; Los, M.; Jonsson-Niedziółka, M.; Krajewska, A.; Szot, K.; Los, J. M.; Niedziolka-Jonsson, J. Antibody Modified Gold Nanoparticles for Fast and Selective, Colorimetric T7 Bacteriophage Detection. Bioconjugate Chem. 2014, 25 (4), 644-648.

66. Lévy, R.; Thanh, N. T. K.; Doty, R. C.; Hussain, I.; Nichols, R. J.; Schiffrin, D. J.; Brust, M.; Fernig, D. G. Rational and Combinatorial Design of Peptide Capping Ligands for Gold Nanoparticles. J. Am. Chem. Soc. 2004, 126 (32), 10076-10084.

67. Lee, Y. F.; Huang, C. C. Colorimetric Assay of Lead Ions in Biological Samples Using a Nanogold-Based Membrane. ACS Appl. Mater. Interfaces 2011, 3 (7), 2747-2754. 
68. Chiu, W. C.; Huang, C. C. Combining Fibrinogen-Conjugated Gold Nanoparticles with a Cellulose Membrane for the Mass Spectrometry-Based Detection of Fibrinolytic-Related Proteins. Anal. Chem. 2013, 85 (14), 6922-6929.

69. Zheng, G.; Polavarapu, L.; Liz-Marzan, L. M.; Pastoriza-Santos, I.; Perez-Juste, J. Gold Nanoparticle-Loaded Filter Paper: a Recyclable Dip-Catalyst for Real-Time Reaction Monitoring by Surface Enhanced Raman Scattering. Chem. Commun. 2015, 51 (22), 45724575 .

70. Wu, S.; Liang, P.P.; Yu, H. X; Xu, X. W; Liu, Y.; Lou, X. H; Xiao, Y. Amplified Single Base-Pair Mismatch Detection via Aggregation of Exonuclease-Sheared Gold Nanoparticles. Anal. Chem. 2014, 86 (7), 3461-3467.

71. Oh, J. H.; Lee, J. S. Designed Hybridization Properties of DNA-Gold Nanoparticle Conjugates for the Ultraselective Detection of a Single-Base Mutation in the Breast Cancer Gene BRCA1. Anal. Chem. 2011, 83 (19), 7364-7370.

72. Mirkin, C. A.; Letsinger, R. L.; Mucic, R. C.; Storhoff, J. J. A DNA-Based Method for Rationally Assembling Nanoparticles into Macroscopic Materials. Nature 1996, 382 (6592), 607-609.

73. Lee, J. S.; Han, M. S.; Mirkin, C. A. Colorimetric Detection of Mercuric Ion $\left(\mathrm{Hg}^{2+}\right)$ in Aqueous Media using DNA-Functionalized Gold Nanoparticles. Angew. Chem. Int. Ed. 2007, 46 (22), 4093-4096.

74. Zhao, W.; Chiuman, W.; Brook, M. A.; Li, Y. F. Simple and Rapid Colorimetric Biosensors Based on DNA Aptamer and Noncrosslinking Gold Nanoparticle Aggregation. ChemBioChem 2007, 8 (7), 727-731.

75. Yager, P.; Edwards, T.; Fu, E.; Helton, K.; Nelson, K.; Tam, M. R.; Weigl, B. H. Microfluidic Diagnostic Technologies for Global Public Health. Nature 2006, 442 (7101), 412-418.

76. Martinez, A. W.; Phillips, S. T.; Butte, M. J.; Whitesides, G. M. Patterned Paper as a Platform for Inexpensive, Low-volume, Portable Bioassays. Angew. Chem. Int. Ed. 2007, 46 (8), 1318-1320.

77. Zhao, W. A.; Ali, M. M.; Aguirre, S. D.; Brook, M. A.; Li, Y. F. Paper-Based Bioassays Using Gold Nanoparticle Colorimetric Probes. Anal. Chem. 2008, 80 (22), 8431-8437.

78. Liu, J. W.; Mazumdar, D.; Lu, Y. A Simple and Sensitive "Dipstick" Test in Serum Based on Lateral Flow Separation of Aptamer-Linked Nanostructures. Angew. Chem. Int. Ed. 2006, 45 (47), 7955-7959.

79. Luckham, R. E.; Brennan, J. D. Bioactive Paper Dipstick Sensors for Acetylcholinesterase Inhibitors Based on Sol-gel/Enzyme/Gold Nanoparticle Composites. Analyst 2010, 135 (8), 2028-2035.

80. Hossain, S. M. Z.; Brennan, J. D. $\beta$-Galactosidase-Based Colorimetric Paper Sensor for Determination of Heavy Metals. Anal. Chem. 2011, 83 (22), 8772-8778. 
81. Alkasir, R. S. J.; Ornatska, M.; Andreescu, S. Colorimetric Paper Bioassay for the Detection of Phenolic Compounds. Anal. Chem. 2012, 84 (22), 9729-9737.

82. Morales-Narváez, E.; Naghdi, T.; Zor, E.; Merkoçi, A. Photoluminescent Lateral-Flow Immunoassay Revealed by Graphene Oxide: Highly Sensitive Paper-Based Pathogen Detection. Anal. Chem. 2015, 87 (16), 8573-8577.

83. Zhou, J. H.; Zheng, Y. P.; Liu, J. J.; Bing, X.; Hua, J. J.; Zhang, H. Y. A Paper-Based Detection Method of Cancer Cells Using the Photo-Thermal Effect of Nanocomposite. $J$. Pharm. Biomed. Anal. 2016, 117, 333-337.

84. Liang P.P.; Yu H.X.; Guntupalli B.; Xiao Y. Paper-Based Device for Rapid Visualization of NADH Based on Dissolution of Gold Nanoparticles." ACS Appl. Mater. Interfaces. 2015, 7 (27), 15023-15030.

85. Weng, C. I.; Cang, J. S.; Chang, J. Y.; Hsiung, T. M.; Unnikrishnan, B.; Hung, Y. L.; Tseng, Y. T.; Li, Y. J.; Shen, Y. W.; Huang, C. C. Detection of Arsenic(III) through Pulsed LaserInduced Desorption/Ionization of Gold Nanoparticles on Cellulose Membranes. Anal. Chem. 2014, 86 (6), 3167-3173.

86. Tsai, T.T.; Shen, S.W.; Cheng, C. M.; Chen, C.F. Paper-Based Tuberculosis Diagnostic Devices with Colorimetric Gold Nanoparticles. Sci. Technol. Adv. Mater. 2013, 14 (4), 044404.

87. Ying, W. NAD $/ \mathrm{NADH}$ and $\mathrm{NADP}^{+} / \mathrm{NADPH}$ in Cellular Functions and Cell Death: Regulation and Biological Consequences. Antioxid. Redox Signal. 2008, 10 (2), 179-206.

88. Belenky, P.; Bogan, K. L.; Brenner, C. $\mathrm{NAD}^{+}$metabolism in health and disease. Trends Biochem. Sci 2007, 32 (1), 12-19.

89. Greenamyre, J. T.; Sherer, T. B.; Betarbet, R.; Panov, A. V. Complex I and Parkinson's disease. IUBMB Life 2001, 52 (3-5), 135-141.

90. Xie, W. J.; Xu, A. S.; Yeung, E. S. Determination of NAD ${ }^{+}$and NADH in a Single Cell under Hydrogen Peroxide Stress by Capillary Electrophoresis. Anal. Chem. 2009, 81 (3), 1280-1284.

91. Lin, S. J.; Guarente, L. Nicotinamide Adenine Dinucleotide, a Metabolic Regulator of Transcription, Longevity and Disease. Curr. Opin. Cell Biol. 2003, 15 (2), 241-246.

92. Heikal, A. A. Intracellular Coenzymes as Natural Biomarkers for Metabolic Activities and Mitochondrial Anomalies. Biomark. Med. 2010, 4 (2), 241-263.

93. Singer, T. P.; Ramsay, R. R.; Ackrell, B. A. C. Deficiencies of NADH and Succinate Dehydrogenases in Degenerative Diseases and Myopathies. Biochimica et Biophysica Acta 1995, 1271 (1), 211-219.

94. Hroudov, J.; Singh, N.; Fišar, Z. Mitochondrial Dysfunctions in Neurodegenerative Diseases: Relevance to Alzheimer's Disease. Biomed. Res. Int. 2014, 2014, 175092. 
95. Birkmayer, J.; Birkmayer, W. The Coenzyme Nicotinamide Adenine Dinucleotide (NADH) as Biological Antidepressive Agent: Experience with 205 Patients. New Trends Clin. Neuropharmacol. 1991, 5, 19-25

96. Ying, W. NAD ${ }^{+}$and NADH in Brain Functions, Brain Diseases and Brain Aging. Front. Biosci. 2007, 12, 1863-1888.

97. Ma, W.; Li, D. W.; Sutherland, T. C.; Li, Y.; Long, Y. T.; Chen, H. Y. Reversible Redox of $\mathrm{NADH}$ and $\mathrm{NAD}^{+}$at a Hybrid Lipid Bilayer Membrane Using Ubiquinone. J. Am. Chem. Soc. 2011, 133 (32), 12366-12369.

98. Ma, W.; Ying, Y. L.; Qin, L. X.; Gu, Z.; Zhou, H.; Li, D. W.; Sutherland, T. C.; Chen, H. Y.; Long, Y. T. Investigating Electron-Transfer Processes Using a Biomimetic Hybrid Bilayer Membrane System. Nat. Protoc. 2013, 8 (3), 439-450.

99. Nachlas, M. M.; Margulies, S. I.; Goldberg, J. D.; Seligman, A. M. The Determination of Lactic Dehydrogenase with a Tetrazolium Salt. Anal. Biochem. 1960, 1, 317-326.

100. Liu, S. F.; Du, Z. F.; Li, P.; Li, F. Sensitive Colorimetric Visualization of Dihydronicotinamide Adenine Dinucleotide Based on Anti-aggregation of Gold Nanoparticles via Boronic Acid-Diol Binding. Biosens. Bioelectron. 2012, 35 (1), 443-446.

101. Jung, S. O.; Ahn, J. Y.; Kim, S.; Yi, S.; Kim, M. H.; Jang, H. H.; Seo, S. H.; Eom, M. S.; Kim, S. K.; Ryu, D. H.; Chang, S. K.; Han, M. S. Fluorescein Derivative-Based, Selective and Sensitive Chemosensor for NADH. Tetrahedron Lett. 2010, 51 (29), 3775-3778.

102. Li, N.; Chen, G. Flow Injection Analysis of Trace Amounts of NADH with Inhibited Chemiluminescent Detection. Talanta 2002, 57 (5), 961-967.

103. Wooten, M.; Gorski, W. Facilitation of NADH Electro-oxidation at Treated Carbon Nanotubes. Anal. Chem. 2010, 82 (4), 1299-1304.

104. Rao, T. N.; Yagi, I.; Miwa, T.; Tryk, D. A.; Fujishima, A. Electrochemical Oxidation of NADH at Highly Boron-Doped Diamond Electrodes. Anal. Chem. 1999, 71 (13), 2506-2511.

105. Yamada, K.; Hara, N.; Shibata, T.; Osago, H.; Tsuchiya, M. The Simultaneous Measurement of Nicotinamide Adenine Dinucleotide and Related Compounds by Liquid Chromatography/Electrospray Ionization Tandem Mass Spectrometry. Anal. Biochem. 2006, 352 (2), 282-285.

106. McComb, R. B.; Bond, L. W.; Burnett, R. W.; Keech, R. C.; Bowers, G. N. Jr. Determination of the Molar Absorptivity of NADH. Clin. Chem. 1976, 22 (2), 141-150.

107. Koning, W. d.; Dam, K. v. A Method for the Determination of Changes of Glycolytic Metabolites in Yeast on a Subsecond Time Scale Using Extraction at Neutral pH. Anal. Biochem. 1992, 204 (1), 118-123.

108. Shlyahovsky, B.; Katz, E.; Xiao, Y.; Pavlov, V.; Willner, I. Optical and Electrochemical Detection of $\mathrm{NADH}$ and of $\mathrm{NAD}^{+}$-Dependent Biocatalyzed Processes by the Catalytic Deposition of Copper on Gold Nanoparticles. Small 2005, 1 (2), 213-216. 
109. Zhang, L.; Li, Y.; Li, D. W.; Jing, C.; Chen, X.; Lv, M.; Huang, Q.; Long, Y. T.; Willner, I. Single Gold Nanoparticles as Real-Time Optical Probes for the Detection of NADHDependent Intracellular Metabolic Enzymatic Pathways. Angew. Chem. Int. Ed. 2011, 50 (30), 6789-6792.

110. Luque de Castro, M. D.; Herrera, M. C. Enzyme Inhibition-Based Biosensors and Biosensing Systems: Questionable Analytical Devices. Biosens. Bioelectron. 2003, 18 (2-3), 279-294.

111. Nwosu, T. N.; Palleschi, G.; Mascini, M. Comparative Studies of Immobilized Enzyme Electrodes Based on the Inhibitory Effect of Nicotine on Choline Oxidase and Acetylcholinesterase. Anal. Lett. 1992, 25 (5), 821-835.

112. Tanei, T.; Morimoto, K.; Shimazu, K.; Kim, S. J.; Tanji, Y.; Taguchi, T.; Tamaki, Y.; Noguchi, S. Association of Breast Cancer Stem Cells Identified by Aldehyde Dehydrogenase 1 Expression with Resistance to Sequential Paclitaxel and Epirubicin-Based Chemotherapy for Breast Cancers. Clin. Cancer. Res. 2009, 15 (12), 4234-4241.

113. Robins, R. K. Nucleoside and Nucleotide Inhibitors of Inosine Monophosphate (IMP) Dehydrogenase as Potential Antitumor Inhibitors. Nucleosides Nucleotides 1982, 1 (1), 3544.

114. Koppaka, V.; Thompson, D. C.; Chen, Y.; Ellermann, M.; Nicolaou, K. C.; Juvonen, R. O.; Petersen, D.; Deitrich, R. A.; Hurley, T. D.; Vasiliou, V. Aldehyde Dehydrogenase Inhibitors: a Comprehensive Review of the Pharmacology, Mechanism of Action, Substrate Specificity, and Clinical Application. Pharmacol. Rev. 2012, 64 (3), 520-539.

115. Arolfo, M. P.; Overstreet, D. H.; Yao, L.; Fan, P.; Lawrence, A. J.; Tao, G.; Keung, W. M.; Vallee, B. L.; Olive, M. F.; Gass, J. T.; Rubin, E.; Anni, H.; Hodge, C. W.; Besheer, J.; Zablocki, J.; Leung, K.; Blackburn, B. K.; Lange, L. G.; Diamond, I. Suppression of Heavy Drinking and Alcohol Seeking by a Selective ALDH-2 Inhibitor. Alcohol Clin Exp Res. 2009, 33 (11), 1935-1944.

116. Yao, L.; Fan, P.; Arolfo, M.; Jiang, Z.; Olive, M. F.; Zablocki, J.; Sun, H. L.; Chu, N.; Lee, J.; Kim, H. Y.; Leung, K.; Shryock, J.; Blackburn, B.; Diamond, I. Inhibition of Aldehyde Dehydrogenase-2 Suppresses Cocaine Seeking by Generating THP, a Cocaine UseDependent Inhibitor of Dopamine Synthesis. Nat. Med. 2010, 16 (9), 1024-1028.

117. Overstreet, D. H.; Knapp, D. J.; Breese, G. R.; Diamond, I. A Selective ALDH-2 Inhibitor Reduces Anxiety in Rats. Pharmacol. Biochem. Behav. 2009, 94 (2), 255-261.

118. Hilton, J. Role of Aldehyde Dehydrogenase in Cyclophosphamide-Resistant L1210 Leukemia. Cancer Res. 1984, 44 (11), 5156-5160.

119. Granchi, C.; Roy, S.; Giacomelli, C.; Macchia, M.; Tuccinardi, T.; Martinelli, A.; Lanza, M.; Betti, L.; Giannaccini, G.; Lucacchini, A.; Funel, N.; Leon, L. G.; Giovannetti, E.; Peters, G. J.; Palchaudhuri, R.; Calvaresi, E. C.; Hergenrother, P. J.; Minutolo, F. Discovery of NHydroxyindole-Based Inhibitors of Human Lactate Dehydrogenase Isoform A (LDH-A) as Starvation Agents Against Cancer Cells. J. Med. Chem. 2011, 54 (6), 1599-1612. 
120. Birkenstock, T.; Liebeke, M.; Winstel, V.; Krismer, B.; Gekeler, C.; Niemiec, M. J.; Bisswanger, H.; Lalk, M.; Peschel, A. Exometabolome Analysis Identifies Pyruvate Dehydrogenase as a Target for the Antibiotic Triphenylbismuthdichloride in Multiresistant Bacterial Pathogens. J. Biol. Chem. 2012, 287 (4), 2887-2895.

121. Degli Esposti, M. Inhibitors of NADH-Ubiquinone Reductase: an Overview. Biochim. Biophys. Acta 1998, 1364 (2), 222-235.

122. Le, A.; Cooper, C. R.; Gouw, A. M.; Dinavahi, R.; Maitra, A.; Deck, L. M.; Royer, R. E.; Vander Jagt, D. L.; Semenza, G. L.; Dang, C. V. Inhibition of Lactate Dehydrogenase A Induces Oxidative Stress and Inhibits Tumor Progression. Proc. Natl. Acad. Sci. U. S. A. 2010, 107 (5), 2037-2042.

123. Allison, S. J.; Knight, J. R. P.; Granchi, C.; Rani, R.; Minutolo, F.; Milner, J.; Phillips, R. M. Identification of LDH-A as a Therapeutic Target for Cancer Cell Killing via (i) p53/NAD(H)-Dependent and (ii) p53-Independent Pathways. Oncogenesis 2014, 3 (5), e102.

124. Hedstrom, L. IMP Dehydrogenase: Structure, Mechanism, and Inhibition. Chem. Rev. 2009, 109 (7), 2903-2928.

125. Deng, S.; Yang, X. J.; Lassus, H.; Liang, S.; Kaur, S.; Ye, Q.; Li, C. S.; Wang, L. P.; Roby, K. F.; Orsulic, S.; Connolly, D. C.; Zhang, Y. C.; Montone, K.; Butzow, R.; Coukos, G.; Zhang, L. Distinct Expression Levels and Patterns of Stem Cell Marker, Aldehyde Dehydrogenase Isoform 1 (ALDH1), in Human Epithelial Cancers. PLoS One 2010, 5 (4), e10277.

126. Zhang, M. G.; Smith, A.; Gorski, W. Carbon Nanotube-Chitosan System for Electrochemical Sensing Based on Dehydrogenase Enzymes. Anal. Chem. 2004, 76 (17), 5045-5050.

127. Kobayashi, Y.; Horikoshi, K. Purification and Properties of NAD-Dependent D-Glucose Dehydrogenase Produced by Alkalophilic Corynebacterium sp. No. 93-1. Agric. Biol. Chem. 1980, 44 (10), 2261-2269.

128. Becker, K. G. The Common Variants/Multiple Disease Hypothesis of Common Complex Genetic Disorders. Med. Hypotheses 2004, 62 (2), 309-317.

129. Suh, Y.; Vijg, J. SNP Discovery in Associating Genetic Variation with Human Disease Phenotypes. Mutat. Res. 2005, 573 (1-2), 41-53.

130. Wang, D. G.; Fan, J. B.; Siao, C. J.; Berno, A.; Young, P.; Sapolsky, R.; Ghandour, G.; Perkins, N.; Winchester, E.; Spencer, J.; Kruglyak, L.; Stein, L.; Hsie, L.; Topaloglou, T.; Hubbell, E.; Robinson, E.; Mittmann, M.; Morris, M. S.; Shen, N.; Kilburn, D.; Rioux, J.; Nusbaum, C.; Rozen, S.; Hudson, T. J.; Lipshutz, R.; Chee, M.; Lander, E. S. Large-Scale Identification, Mapping, and Genotyping of Single-Nucleotide Polymorphisms in the Human Genome. Science 1998, 280 (5366), 1077-1082.

131. Cruchaga, C.; Kauwe, J. S.; Mayo, K.; Spiegel, N.; Bertelsen, S.; Nowotny, P.; Shah, A. R.; Abraham, R.; Hollingworth, P.; Harold, D.; Owen, M. M.; Williams, J.; Lovestone, S.; Peskind, E. R.; Li, G.; Leverenz, J. B.; Galasko, D.; Morris, J. C.; Fagan, A. M.; Holtzman, 
D. M.; Goate, A. M. SNPs Associated with Cerebrospinal Fluid Phospho-Tau Levels Influence Rate of Decline in Alzheimer's Disease. PLoS Genet. 2010, 6 (9), e1001101.

132. Simon-Sanchez, J.; Scholz, S.; del Mar Matarin, M.; Fung, H. C.; Hernandez, D.; Gibbs, J. R.; Britton, A.; Hardy, J.; Singleton, A. Genomewide SNP Assay Reveals Mutations Underlying Parkinson Disease. Hum. Mutat. 2008, 29 (2), 315-322.

133. Dayeh, T. A.; Olsson, A. H.; Volkov, P.; Almgren, P.; Ronn, T.; Ling, C. Identification of CpG-SNPs Associated with Type 2 Diabetes and Differential DNA Methylation in Human Pancreatic Islets. Diabetologia 2013, 56 (5), 1036-1046.

134.Erichsen, H. C.; Chanock, S. J. SNPs in Cancer Research and Treatment. Br. J. Cancer 2004, 90 (4), 747-751.

135. Chakravarti, A. Single Nucleotide Polymorphisms: . . to a Future of Genetic Medicine. Nature 2001, 409 (6822), 822-823.

136. Lai, E. Application of SNP Technologies in Medicine: Lessons Learned and Future Challenges. Genome Res. 2001, 11 (6), 927-929.

137. Matsuzaki, H.; Dong, S. L.; Loi, H.; Di, X. J.; Liu, G. Y.; Hubbell, E.; Law, J.; Berntsen, T.; Chadha, M.; Hui, H.; Yang, G.; Kennedy, G. C.; Webster, T. A.; Cawley, S.; Walsh, P. S.; Jones, K. W.; Fodor, S. P. A.; Mei, R. Genotyping Over 100,000 SNPs on a Pair of Oligonucleotide Arrays. Nat. Methods 2004, 1 (2), 109-111.

138. Kurg, A.; Tõnisson, N.; Georgiou, I.; Shumaker, J.; Tollett, J.; Metspalu, A. Arrayed Primer Extension: Solid-Phase Four-Color DNA Resequencing and Mutation Detection Technology. Genet. Test. 2000, 4 (1), 1-7.

139. Kuppuswamy, M. N.; Hoffmann, J. W.; Kasper, C. K.; Spitzer, S. G.; Groce, S. L.; Bajaj, S. P. Single Nucleotide Primer Extension to Detect Genetic Diseases: Experimental Application to Hemophilia B (factor IX) and Cystic Fibrosis Genes. Proc. Natl. Acad. Sci. U. S. A. 1991, 88 (4), 1143-1147.

140. Landegren, U.; Kaiser, R.; Sanders, J.; Hood, L. A Ligase-Mediated Gene Detection Technique. Science 1988, 241 (4869), 1077-1080.

141. Lyamichev, V.; Mast, A. L.; Hall, J. G.; Prudent, J. R.; Kaiser, M. W.; Takova, T.; Kwiatkowski, R. W.; Sander, T. J.; de Arruda, M.; Arco, D. A.; Neri, B. P.; Brow, M. A. D. Polymorphism Identification and Quantitative Detection of Genomic DNA by Invasive Cleavage of Oligonucleotide Probes. Nat. Biotechnol. 1999, 17 (3), 292-296.

142. Lee, L. G.; Connell, C. R.; Bloch, W. Allelic Discrimination by Nick-Translation PCR with Fluorogenic Probes. Nucleic Acids Res. 1993, 21 (16), 3761-3766.

143. Howell, W. M.; Jobs, M.; Gyllensten, U.; Brookes, A. J. Dynamic Allele-Specific Hybridization. A new method for scoring single nucleotide polymorphisms. Nat. Biotechnol. 1999, $17(1), 87-88$. 
144. Tyagi, S.; Bratu, D. P.; Kramer, F. R. Multicolor Molecular Beacons for Allele Discrimination. Nat. Biotechnol. 1998, 16 (1), $49-53$.

145. Kolpashchikov, D. M. Binary Malachite Green Aptamer for Fluorescent Detection of Nucleic Acids. J. Am. Chem. Soc. 2005, 127 (36), 12442-12443.

146. Kolpashchikov, D. M. A Binary DNA Probe for Highly Specific Nucleic Acid Recognition. J. Am. Chem. Soc. 2006, 128 (32), 10625-10628.

147. Xiao, Y.; Plakos, K. J. I.; Lou, X.; White, R. J.; Qian, J.; Plaxco, K. W.; Soh, H. T. Fluorescence Detection of Single-Nucleotide Polymorphisms with a Single, SelfComplementary, Triple-Stem DNA Probe. Angew. Chem. 2009, 121 (24), 4418-4422.

148. Sauer, S.; Reinhardt, R.; Lehrach, H.; Gut, I. G. Single-Nucleotide Polymorphisms: Analysis by Mass Spectrometry. Nat. Protoc. 2006, 1 (4), 1761-1771.

149. Hu, R.; Wu, Z. S.; Zhang, S.; Shen, G. L.; Yu, R. Robust Electrochemical System for Screening Single Nucleotide Polymorphisms. Chem. Commun. 2011, 47 (4), 1294-1296.

150. Knapp, L. A. Single Nucleotide Polymorphism Screening with Denaturing Gradient Gel Electrophoresis. Methods Mol Biol. 2009, 578, 137-151.

151. Vogt, V. M. Purification and Further Properties of Single-Strand-Specific Nuclease from Aspergillus oryzae. Eur. J. Biochem. 1973, 33 (1), 192-200.

152. Alivisatos, A. P.; Johnsson, K. P.; Peng, X.; Wilson, T. E.; Loweth, C. J.; Bruchez, M. P.; Schultz, P. G. Organization of 'Nanocrystal Molecules' Using DNA. Nature 1996, 382 (6592), 609-611.

153. Niemeyer, C. M.; Simon, U. DNA-Based Assembly of Metal Nanoparticles. Eur. J. Inorg. Chem. 2005, 2005 (18), 3641-3655.

154. Wang, X. Y.; Zou, M. J.; Huang, H. D.; Ren, Y. Q.; Li, L. M.; Yang, X. D.; Li, N. Gold Nanoparticle Enhanced Fluorescence Anisotropy for the Assay of Single Nucleotide Polymorphisms (SNPs) Based on Toehold-Mediated Strand-Displacement Reaction. Biosens. Bioelectron. 2013, 41, 569-575.

155. Fang, W. F.; Chen, W. J.; Yang, J. T. Colorimetric Determination of DNA Concentration and Mismatches Using Hybridization-Mediated Growth of Gold Nanoparticle Probes. Sensors Actuators B: Chem. 2014, 192, 77-82.

156. Sato, K.; Hosokawa, K.; Maeda, M. Non-cross-linking Gold Nanoparticle Aggregation as a Detection Method for Single-Base Substitutions. Nucleic Acids Res. 2005, 33 (1), e4.

157. Li, H.X.; Rothberg, L. J. Label-Free Colorimetric Detection of Specific Sequences in Genomic DNA Amplified by the Polymerase Chain Reaction. J. Am. Chem. Soc. 2004, 126 (35), 10958-10961. 
158. Xu, W.; Xue, X. J.; Li, T. H.; Zeng, H. Q.; Liu, X. G. Ultrasensitive and Selective Colorimetric DNA Detection by Nicking Endonuclease Assisted Nanoparticle Amplification. Angew. Chem. Int. Ed. 2009, 48 (37), 6849-6852.

159. Li, J. S.; Deng, T.; Chu, X.; Yang, R. H.; Jiang, J. H.; Shen, G. L.; Yu, R. Q. Rolling Circle Amplification Combined with Gold Nanoparticle Aggregates for Highly Sensitive Identification of Single-Nucleotide Polymorphisms. Anal. Chem. 2010, 82 (7), 2811-2816.

160. Shen, W.; Deng, H. M.; Gao, Z. Q. Gold Nanoparticle-Enabled Real-Time Ligation Chain Reaction for Ultrasensitive Detection of DNA. J. Am. Chem. Soc. 2012, 134 (36), 1467814681.

161. Cederquist, K. B.; Keating, C. D. Hybridization Efficiency of Molecular Beacons Bound to Gold Nanowires: Effect of Surface Coverage and Target Length. Langmuir 2010, 26 (23), 18273-18280.

162. Zu, Y. B.; Gao, Z. Q. Facile and Controllable Loading of Single-Stranded DNA on Gold Nanoparticles. Anal. Chem. 2009, 81 (20), 8523-8528.

163. Cederquist, K. B.; Stoermer Golightly, R.; Keating, C. D. Molecular Beacon-Metal Nanowire Interface: Effect of Probe Sequence and Surface Coverage on Sensor Performance. Langmuir 2008, 24 (16), 9162-9171.

164. Seferos, D. S.; Prigodich, A. E.; Giljohann, D. A.; Patel, P. C.; Mirkin, C. A. Polyvalent DNA Nanoparticle Conjugates Stabilize Nucleic Acids. Nano Lett. 2009, 9 (1), 308-311.

165. Peterson, A. W.; Heaton, R. J.; Georgiadis, R. M. The Effect of Surface Probe Density on DNA Hybridization. Nucleic Acids Res. 2001, 29 (24), 5163-5168.

166. Li, F.; Zhang, H. Q.; Dever, B.; Li, X. F.; Le, X. C. Thermal Stability of DNA Functionalized Gold Nanoparticles. Bioconjugate Chem. 2013, 24 (11), 1790-1797.

167. Liu, C. W.; Huang, C. C.; Chang, H. T. Control over Surface DNA Density on Gold Nanoparticles Allows Selective and Sensitive Detection of Mercury(II). Langmuir 2008, 24 (15), 8346-8350.

168. Wernette, D. P.; Mead, C.; Bohn, P. W.; Lu, Y. Surface Immobilization of Catalytic Beacons Based on Ratiometric Fluorescent DNAzyme Sensors: a Systematic Study. Langmuir 2007, 23 (18), 9513-9521.

169. Hurst, S. J.; Lytton-Jean, A. K. R.; Mirkin, C. A. Maximizing DNA Loading on a Range of Gold Nanoparticle Sizes. Anal. Chem. 2006, 78 (24), 8313-8318.

170. Seela, F.; Ding, P.; Budow, S. DNA Gold Nanoparticle Conjugates Incorporating Thiooxonucleosides: 7-Deaza-6-thio-2'-deoxyguanosine as Gold Surface Anchor. Bioconjugate Chem. 2011, 22 (4), 794-807.

171. Gong, P.; Lee, C. Y.; Gamble, L. J.; Castner, D. G.; Grainger, D. W. Hybridization Behavior of Mixed DNA/Alkylthiol Monolayers on Gold: Characterization by Surface Plasmon Resonance and ${ }^{32} \mathrm{P}$ Radiometric Assay. Anal. Chem. 2006, 78 (10), 3326-3334. 
172. Arinaga, K.; Rant, U.; Knežević, J.; Pringsheim, E.; Tornow, M.; Fujita, S.; Abstreiter, G.; Yokoyama, N. Controlling the Surface Density of DNA on Gold by Electrically Induced Desorption. Biosens. Bioelectron. 2007, 23 (3), 326-331.

173. Herne, T. M.; Tarlov, M. J. Characterization of DNA Probes Immobilized on Gold Surfaces. J. Am. Chem. Soc. 1997, 119 (38), 8916-8920.

174. Liu, J. W. Adsorption of DNA onto Gold Nanoparticles and Graphene Oxide: Surface Science and Applications. Phys. Chem. Chem. Phys. 2012, 14 (30), 10485-10496.

175. Östblom, M.; Liedberg, B.; Demers, L. M.; Mirkin, C. A. On the Structure and Desorption Dynamics of DNA Bases Adsorbed on Gold: A Temperature-Programmed Study. J Phys Chem B. 2005, 109 (31), 15150-15160.

176. Pei, H.; Li, F.; Wan, Y.; Wei, M.; Liu, H.; Su, Y.; Chen, N.; Huang, Q.; Fan, C. H. Designed Diblock Oligonucleotide for the Synthesis of Spatially Isolated and Highly Hybridizable Functionalization of DNA-Gold Nanoparticle Nanoconjugates. J. Am. Chem. Soc. 2012, 134 (29), 11876-11879.

177. Boozer, C.; Chen, S. F.; Jiang, S. Y. Controlling DNA Orientation on Mixed ssDNA/OEG SAMs. Langmuir 2006, 22 (10), 4694-4698.

178. Keighley, S. D.; Li, P.; Estrela, P.; Migliorato, P. Optimization of DNA Immobilization on Gold Electrodes for Label-Free Detection by Electrochemical Impedance Spectroscopy. Biosens. Bioelectron. 2008, 23 (8), 1291-1297.

179. Park, S.; Brown, K. A.; Hamad-Schifferli, K. Changes in Oligonucleotide Conformation on Nanoparticle Surfaces by Modification with Mercaptohexanol. Nano Lett. 2004, 4 (10), 1925-1929.

180. Liu, T.; Zhao, J.; Zhang, D. M.; Li, G.X. Novel Method to Detect DNA Methylation Using Gold Nanoparticles Coupled with Enzyme-Linkage Reactions. Anal. Chem. 2010, 82 (1), 229-233.

181. MacDairmid, A. R.; Gallagher, M. C.; Banks, J. T. Structure of Dithiothreitol Monolayers on $\mathrm{Au}(111)$. J. Phys. Chem. B 2003, 107 (36), 9789-9792.

182. Wu, J.; Campuzano, S.; Halford, C.; Haake, D. A.; Wang, J. Ternary Surface Monolayers for Ultrasensitive (Zeptomole) Amperometric Detection of Nucleic Acid Hybridization without Signal Amplification. Anal. Chem. 2010, 82 (21), 8830-8837.

183. Johnson, E. L. Content and Distribution of Erythroxylum coca Leaf Alkaloids. Ann. Bot. 1995, 76 (4), 331-335.

184. Loper, K. A. Clinical Toxicology of Cocaine. Med. Toxicol. Adverse Drug Exp. 1989, 4 (3), 174-185.

185. Center for Behavioral Health Statistics and Quality. (2015). Behavioral health trends in the United States: Results from the 2014 National Survey on Drug Use and Health (HHS Publication No. SMA 15-4927, NSDUH Series H-50). 
186. Riezzo, I.; Fiore, C.; De Carlo, D.; Pascale, N.; Neri, M.; Turillazzi, E.; Fineschi, V. Side Effects of Cocaine Abuse: Multiorgan Toxicity and Pathological Consequences. Curr. Med. Chem. 2012, 19 (33), 5624-5646.

187. Lutiger, B.; Graham, K.; Einarson, T. R.; Koren, G. Relationship Between Gestational Cocaine Use and Pregnancy Outcome: A Meta-Analysis. Teratology 1991, 44 (4), 405-414.

188. Marchei, E.; Colone, P.; Nastasi, G. G.; Calabrò, C.; Pellegrini, M.; Pacifici, R.; Zuccaro, P.; Pichini, S. On-site Screening and GC-MS Analysis of Cocaine and Heroin Metabolites in Body-Packers Urine. J. Pharm. Biomed. Anal. 2008, 48 (2), 383-387.

189. Moody, D. E.; Spanbauer, A. C.; Taccogno, J. L.; Smith, E. K. Comparative Analysis of Sweat Patches for Cocaine (and Metabolites) by Radioimmunoassay and Gas Chromatography-Positive Ion Chemical Ionization-Mass Spectrometry. J. Anal. Toxicol. 2004, 28 (2), 86-93.

190. Cone, E. J. Validity Testing of Commercial Urine Cocaine Metabolite assays: III. Evaluation of an Enzyme-Linked Immunosorbent Assay (ELISA) for Detection of Cocaine and Cocaine Metabolite. J. Forensic Sci. 1989, 34 (4), 991-995.

191. López, P.; Martello, S.; Bermejo, A. M.; De Vincenzi, E.; Tabernero, M. J.; Chiarotti, M. Validation of ELISA Screening and LC-MS/MS Confirmation Methods for Cocaine in Hair After Simple Extraction. Anal. Bioanal. Chem. 2010, 397 (4), 1539-1548.

192. DiGregorio, G. J.; Ferko, A. P.; Barbieri, E. J.; Ruch, E. K.; Chawla, H.; Keohane, D.; Rosenstock, R.; Aldano, A. Determination of Cocaine Usage in Pregnant Women by a Urinary EMIT Drug Screen and GC-MS Analyses. J. Anal. Toxicol. 1994, 18 (5), 247-250.

193. Armbruster, D. A.; Schwarzhoff, R. H.; Hubster, E. C.; Liserio, M. K. Enzyme Immunoassay, Kinetic Microparticle Immunoassay, Radioimmunoassay, and Fluorescence Polarization Immunoassay Compared for Drugs-of-Abuse Screening. Clin. Chem. 1993, 39 (10), 2137-2146.

194. Law, B.; Moffat, A. C. The Evaluation of an Homogeneous Enzyme Immunoassay (Emit) and Radioimmunoassay for Barbiturates. J Forensic Sci Soc. 1981, 21 (1), 55-66.

195. Moeller, K. E.; Lee, K. C.; Kissack, J. C. Urine Drug Screening: Practical Guide for Clinicians. Mayo Clin. Proc. 2008, 83 (1), 66-76.

196. Krasowski, M. D.; Pizon, A. F.; Siam, M. G.; Giannoutsos, S.; Iyer, M.; Ekins, S. Using Molecular Similarity to Highlight the Challenges of Routine Immunoassay-Based Drug of Abuse/Toxicology Screening in Emergency Medicine. BMC Emerg. Med. 2009, 9 (1), 1-18.

197. Phillips, D. L.; Tebbett, I. R.; Bertholf, R. L. Comparison of HPLC and GC-MS for Measurement of Cocaine and Metabolites in Human Urine. J. Anal. Toxicol. 1996, 20 (5), 305-308.

198. Kapur, B. M. Drug-Testing Methods and Clinical Interpretations of Test Results. Bull. Narc. 1993, 45 (2), 115-154. 
199. Mokhtarzadeh, A.; Dolatabadi JE.; Abnous, K.; de la Guardia, M.; Ramezani, M. Nanomaterial-Based Cocaine Aptasensors. Biosens. Bioelectron. 2015, 68, 95-106.

200. Tuerk, C.; Gold, L. Systematic Evolution of Ligands by Exponential Enrichment: RNA Ligands to Bacteriophage T4 DNA Polymerase. Science 1990, 249 (4968), 505-510.

201. Ellington, A. D.; Szostak, J. W. In Vitro Selection of RNA Molecules that Bind Specific Ligands. Nature 1990, 346 (6287), 818-822.

202. Jellinek, D.; Lynott, C. K.; Rifkin, D. B.; Janjić, N. High-Affinity RNA Ligands to Basic Fibroblast Growth Factor Inhibit Receptor Binding. Proc. Natl. Acad. Sci. U. S. A. 1993, 90 (23), 11227-11231.

203. Haller, A. A.; Sarnow, P. In Vitro Selection of a 7-Methyl-guanosine Binding RNA that Inhibits Translation of Capped mRNA Molecules. Proc. Natl. Acad. Sci. U. S. A. 1997, 94 (16), 8521-8526.

204. Geiger, A.; Burgstaller, P.; von der Eltz, H.; Roeder, A.; Famulok, M. RNA Aptamers That Bind L-Arginine with Sub-Micromolar Dissociation Constants and High Enantioselectivity. Nucleic Acids Res. 1996, 24 (6), 1029-1036.

205. Zhang, P.; Zhao, N.X.; Zeng, Z. H.; Chang, C. C.; Zu, Y. L. Combination of an Aptamer Probe to CD4 and Antibodies for Multicolored Cell Phenotyping. Am. J. Clin. Pathol. 2010, 134 (4), 586-593.

206. Woo, H. M.; Kim, K. S.; Lee, J. M.; Shim, H. S.; Cho, S. J.; Lee, W. K.; Ko, H. W.; Keum, Y. S.; Kim, S. Y.; Pathinayake, P.; Kim, C. J.; Jeong, Y. J. Single-Stranded DNA Aptamer that Specifically Binds to the Influenza Virus NS1 Protein Suppresses Interferon Antagonism. Antiviral Res. 2013, 100 (2), 337-345.

207. Zhou, J. H.; Li, H. T.; Li, S.; Zaia, J.; Rossi, J. J. Novel Dual Inhibitory Function AptamersiRNA Delivery System for HIV-1 Therapy. Mol. Ther. 2008, 16 (8), 1481-1489.

208. Sefah, K.; Shangguan, D. H.; Xiong, X. L.; O'Donoghue, M. B.; Tan, W. H. Development of DNA Aptamers Using Cell-SELEX. Nat. Protoc. 2010, 5 (6), 1169-1185.

209. Tan, W.H.; Donovan, M. J.; Jiang, J. H. Aptamers from Cell-Based Selection for Bioanalytical Applications. Chem. Rev. 2013, 113 (4), 2842-2862.

210. Chen, H. W.; Medley, C. D.; Sefah, K.; Shangguan, D.; Tang, Z. W.; Meng, L.; Smith, J. E.; Tan, W. H. Molecular Recognition of Small-Cell Lung Cancer Cells Using Aptamers. ChemMedChem 2008, 3 (6), 991-1001.

211. Shangguan, D. H,; Meng, L.; Cao, Z. C.; Xiao, Z. Y.; Fang, X. H.; Li, Y.; Cardona, D.; Witek, R. P.; Liu, C.; Tan, W. H. Identification of Liver Cancer-Specific Aptamers Using Whole Live Cells. Anal. Chem. 2008, 80 (3), 721-728.

212. Stojanovic, M. N.; de Prada, P.; Landry, D. W. Fluorescent Sensors Based on Aptamer SelfAssembly. J. Am. Chem. Soc. 2000, 122 (46), 11547-11548. 
213. Stojanovic, M. N.; de Prada, P.; Landry, D. W. Aptamer-Based Folding Fluorescent Sensor for Cocaine. J. Am. Chem. Soc. 2001, 123 (21), 4928-4931.

214. Rajendran, M.; Ellington, A. D. Selection of Fluorescent Aptamer Beacons that Light up in the Presence of Zinc. Anal. Bioanal. Chem. 2008, 390 (4), 1067-1075.

215. Jayasena, S. D. Aptamers: An Emerging Class of Molecules That Rival Antibodies in Diagnostics. Clin. Chem. 1999, 45 (9), 1628-1650.

216. Ng, A.; Chinnappan, R.; Eissa, S.; Liu, H. C.; Tlili, C.; Zourob, M. Selection, Characterization, and Biosensing Application of High Affinity Congener-Specific Microcystin-Targeting Aptamers. Environ. Sci. Technol. 2012, 46 (19), 10697-10703.

217.Hicke, B. J.; Stephens, A. W.; Gould, T.; Chang, Y. F.; Lynott, C. K.; Heil, J.; Borkowski, S.; Hilger, C. S.; Cook, G.; Warren, S.; Schmidt, P. G. Tumor Targeting by an Aptamer. J. Nucl. Med. 2006, 47 (4), 668-678.

218. Farokhzad, O. C.; Jon, S.; Khademhosseini, A.; Tran, T.-N. T.; LaVan, D. A.; Langer, R. Nanoparticle-Aptamer Bioconjugates: A New Approach for Targeting Prostate Cancer Cells. Cancer Res. 2004, 64 (21), 7668-7672.

219. Vinores, S. A. Pegaptanib in the Treatment of Wet, Age-Related Macular Degeneration. Int. J. Nanomedicine 2006, 1 (3), 263-268.

220. Ng, E. W. M.; Shima, D. T.; Calias, P.; Cunningham, E. T.; Guyer, D. R.; Adamis, A. P. Pegaptanib, a Targeted Anti-VEGF Aptamer for Ocular Vascular Disease. Nat. Rev. Drug Discov. 2006, 5 (2), 123-132.

221. Zhou, B.; Wang, B. Pegaptanib for the Treatment of Age-Related Macular Degeneration. Exp. Eye Res. 2006, 83 (3), 615-619.

222. Bagalkot, V.; Zhang, L. F.; Levy-Nissenbaum, E.; Jon, S. Y.; Kantoff, P. W.; Langer, R.; Farokhzad, O. C. Quantum Dot-Aptamer Conjugates for Synchronous Cancer Imaging, Therapy, and Sensing of Drug Delivery Based on Bi-Fluorescence Resonance Energy Transfer. Nano Lett. 2007, 7 (10), 3065-3070.

223. Que-Gewirth, N. S.; Sullenger, B. A. Gene Therapy Progress and Prospects: RNA Aptamers. Gene Ther. 2007, 14 (4), 283-291.

224. Browning, A. C.; Amoaku, W. M.; Dua, H. S. Treatment of Age-Related Macular Degeneration. J. R. Soc. Med. 2004, 97 (4), 166-169.

225. Shlyahovsky, B.; Li, D.; Weizmann, Y.; Nowarski, R.; Kotler, M.; Willner, I. Spotlighting of Cocaine by an Autonomous Aptamer-Based Machine. J. Am. Chem. Soc. 2007, 129 (13), 3814-3815.

226. He, J. L.; Wu, Z. S.; Zhou, H.; Wang, H. Q.; Jiang, J. H.; Shen, G. L.; Yu, R. Q. Fluorescence Aptameric Sensor for Strand Displacement Amplification Detection of Cocaine. Anal. Chem. 2010, 82 (4), 1358-1364. 
227. Zhang, C. Y.; Johnson, L. W. Single Quantum-Dot-Based Aptameric Nanosensor for Cocaine. Anal. Chem. 2009, 81 (8), 3051-3055.

228. Liu, J. W.; Lu, Y. Fast Colorimetric Sensing of Adenosine and Cocaine Based on a General Sensor Design Involving Aptamers and Nanoparticles. Angew. Chem. Int. Ed. 2006, 45 (1), 90-94.

229. Stojanovic, M. N.; Landry, D. W. Aptamer-Based Colorimetric Probe for Cocaine. J. Am. Chem. Soc. 2002, 124 (33), 9678-9679.

230. Elbaz, J.; Shlyahovsky, B.; Li, D.; Willner, I. Parallel Analysis of Two Analytes in Solutions or on Surfaces by Using a Bifunctional Aptamer: Applications for Biosensing and Logic Gate Operations. ChemBioChem 2008, 9 (2), 232-239.

231. Yan, X. L.; Cao, Z. J.; Lau, C.; Lu, J. Z. DNA Aptamer Folding on Magnetic Beads for Sequential Detection of Adenosine and Cocaine by Substrate-Resolved Chemiluminescence Technology. Analyst 2010, 135 (9), 2400-2407.

232. Li, T.; Li, B.; Dong, S. J. Adaptive Recognition of Small Molecules by Nucleic Acid Aptamers through a Label-Free Approach. Chem. Eur. J. 2007, 13 (23), 6718-6723.

233. Hua, M.; Tao, M. L.; Wang, P.; Zhang, Y. F.; Wu, Z. S.; Chang, Y. B.; Yang, Y. H. LabelFree Electrochemical Cocaine Aptasensor Based on a Target-Inducing Aptamer Switching Conformation. Anal. Sci. 2010, 26 (12), 1265-1270.

234. Zhang, D. W.; Nie, J.; Zhang, F. T.; Xu, L.; Zhou, Y. L.; Zhang, X. X. Novel Homogeneous Label-Free Electrochemical Aptasensor Based on Functional DNA Hairpin for Target Detection. Anal. Chem. 2013, 85 (19), 9378-9382.

235. White, R. J.; Phares, N.; Lubin, A. A.; Xiao, Y.; Plaxco, K. W. Optimization of Electrochemical Aptamer-Based Sensors via Optimization of Probe Packing Density and Surface Chemistry. Langmuir 2008, 24 (18), 10513-10518.

236. Chen, J. W.; Jiang, J. H.; Gao, X.; Liu, G. K.; Shen, G. L.; Yu, R. Q. A New Aptameric Biosensor for Cocaine Based on Surface-Enhanced Raman Scattering Spectroscopy. Chemistry 2008, 14 (27), 8374-8382.

237. Zou, R. X.; Lou, X. H.; Ou, H. C.; Zhang, Y.; Wang, W. J.; Yuan, M.; Guan, M.; Luo, Z. F.; Liu, Y. Y. Highly Specific Triple-Fragment Aptamer for Optical Detection of Cocaine. RSC Adv. 2012, 2 (11), 4636-4638.

238. Zhao, T.; Liu, R.; Ding, X. F.; Zhao, J. C.; Yu, H. X.; Wang, L.; Xu, Q.; Wang, X.; Lou, X. H.; He, M.; Xiao, Y. Nanoprobe-Enhanced, Split Aptamer-Based Electrochemical Sandwich Assay for Ultrasensitive Detection of Small Molecules. Anal. Chem. 2015, 87 (15), 77127719.

239. Zuo, X. L.; Xiao, Y.; Plaxco, K. W. High Specificity, Electrochemical Sandwich Assays Based on Single Aptamer Sequences and Suitable for the Direct Detection of SmallMolecule Targets in Blood and Other Complex Matrices. J. Am. Chem. Soc. 2009, 131 (20), 6944-6945. 
240. Freeman, R.; Li, Y.; Tel-Vered, R.; Sharon, E.; Elbaz, J.; Willner, I. Self-Assembly of Supramolecular Aptamer Structures for Optical or Electrochemical Sensing. Analyst 2009, 134 (4), 653-656.

241. Golub, E.; Pelossof, G.; Freeman, R.; Zhang, H.; Willner, I. Electrochemical, Photoelectrochemical, and Surface Plasmon Resonance Detection of Cocaine Using Supramolecular Aptamer Complexes and Metallic or Semiconductor Nanoparticles. Anal. Chem. 2009, 81 (22), 9291-9298.

242. Liu, X.; Freeman, R.; Willner, I. Amplified Fluorescence Aptamer-Based Sensors Using Exonuclease III for the Regeneration of the Analyte. Chem. Eur. J. 2012, 18 (8), 2207-2211.

243. Du, Y.; Li, B. L.; Guo, S. J.; Zhou, Z. X.; Zhou, M.; Wang, E. K.; Dong, S. J. GQuadruplex-Based DNAzyme for Colorimetric Detection of Cocaine: Using Magnetic Nanoparticles as the Separation and Amplification Element. Analyst 2011, 136 (3), 493-497.

244. Zhang, J.; Wang, L. H.; Pan, D.; Song, S. P.; Boey, F. Y. C.; Zhang, H.; Fan, C. H. Visual Cocaine Detection with Gold Nanoparticles and Rationally Engineered Aptamer Structures. Small 2008, 4 (8), 1196-1200.

245. Park, S. J.; Taton, T. A.; Mirkin, C. A. Array-Based Electrical Detection of DNA with Nanoparticle Probes. Science 2002, 295 (5559), 1503-1506.

246. Ivanova, O. S.; Zamborini, F. P. Electrochemical Size Discrimination of Gold Nanoparticles Attached to Glass/Indium-Tin-Oxide Electrodes by Oxidation in Bromide-Containing Electrolyte. Anal. Chem. 2010, 82 (13), 5844-5850.

247. Domingos, R. F.; Baalousha, M. A.; Ju-Nam, Y.; Reid, M. M.; Tufenkji, N.; Lead, J. R.; Leppard, G. G.; Wilkinson, K. J. Characterizing Manufactured Nanoparticles in the Environment: Multimethod Determination of Particle Sizes. Environ. Sci. Technol. 2009, 43 (19), 7277-7284.

248. Cifuentes, A.; Bernal, J. L.; Diez-Masa, J. C. Determination of Critical Micelle Concentration Values Using Capillary Electrophoresis Instrumentation. Anal. Chem. 1997, $69(20), 4271-4274$.

249. Mortier, T.; Persoons, A.; Verbiest, T. Oxidation of Solid Gold in Chloroform Solutions of Cetyltrimethylammonium Bromide. Inorg. Chem. Commun. 2005, 8 (12), 1075-1077.

250. Wu, Z. C.; Chen, Z. H.; Du, X.; Logan, J. M.; Sippel, J.; Nikolou, M.; Kamaras, K.; Reynolds, J. R.; Tanner, D. B.; Hebard, A. F.; Rinzler, A. G. Transparent, Conductive Carbon Nanotube Films. Science 2004, 305 (5688), 1273-1276.

251. Lee, J. H.; Kong, B. S.; Baek, Y. K.; Yang, S. B.; Jung, H. T. Tin Nanoparticle Thin Film Electrodes Fabricated by the Vacuum Filtration Method for Enhanced Battery Performance. Nanotechnology 2009, 20 (23), 235203.

252. Carrilho, E.; Martinez, A. W.; Whitesides, G. M. Understanding Wax Printing: A Simple Micropatterning Process for Paper-Based Microfluidics. Anal. Chem. 2009, 81 (16), 70917095. 
253. Dorshow, R.; Briggs, J.; Bunton, C. A.; Nicoli, D. F. Dynamic Light Scattering from Cetyltrimethylammonium Bromide Micelles. Intermicellar Interactions at Low Ionic Strengths. J. Phys. Chem. 1982, 86 (13), 2388-2395.

254. Deegan, R. D.; Bakajin, O.; Dupont, T. F.; Huber, G.; Nagel, S. R.; Witten, T. A. Capillary Flow as the Cause of Ring Stains from Dried Liquid Drops. Nature 1997, 389 (6653), 827829.

255. Mills, R. Self-Diffusion in Normal and Heavy Water in the Range 1-45.deg. J. Phys. Chem. 1973, 77 (5), 685-688.

256. Lindman, B.; Puyal, M. C.; Kamerka, N.; Rymden, R.; Stilbs, P. Micelle Formation of Anionic and Cationic Surfactants from Fourier Transform Hydrogen-1 and Lithium-7 Nuclear Magnetic Resonance and Tracer Self-Diffusion Studies. J. Phys. Chem. 1984, 88 (21), 5048-5057.

257. Otto, W. H.; Britten, D. J.; Larive, C. K. NMR Diffusion Analysis of Surfactant-Humic Substance Interactions. J. Colloid Interface Sci. 2003, 261 (2), 508-513.

258. Wu, Q.; Maskus, M.; Pariente, F.; Tobalina, F.; Fernández, V. M.; Lorenzo, E.; Abruña, H. D. Electrocatalytic Oxidation of NADH at Glassy Carbon Electrodes Modified with Transition Metal Complexes Containing 1,10-Phenanthroline-5,6-dione Ligands. Anal. Chem. 1996, 68 (20), 3688-3696.

259. Pariente, F.; Lorenzo, E.; Tobalina, F.; Abruna, H. D. Aldehyde Biosensor Based on the Determination of NADH Enzymically Generated by Aldehyde Dehydrogenase. Anal. Chem. 1995, 67 (21), 3936-3944.

260. Teymourian, H.; Salimi, A.; Hallaj, R. Low potential Detection of NADH Based on $\mathrm{Fe}_{3} \mathrm{O}_{4}$ Nanoparticles/Multiwalled Carbon Nanotubes Composite: Fabrication of Integrated Dehydrogenase-Based Lactate Biosensor. Biosens. Bioelectron. 2012, 33 (1), 60-68.

261. Álvarez-González, M. I.; Saidman, S. B.; Lobo-Castañón, M. J.; Miranda-Ordieres, A. J.; Tuñón-Blanco, P. Electrocatalytic Detection of NADH and Glycerol by NAD ${ }^{+}$-Modified Carbon Electrodes. Anal. Chem. 2000, 72 (3), 520-527.

262. Narvaez Villarrubia, C. W.; Rincon, R. A.; Radhakrishnan, V. K.; Davis, V.; Atanassov, P. Methylene Green Electrodeposited on SWNTs-Based "Bucky" Papers for NADH and LMalate Oxidation. ACS Appl. Mater. Interfaces 2011, 3 (7), 2402-2409.

263. Won, B. Y.; Shin, S. C.; Chung, W. Y.; Shin, S.; Cho, D. Y.; Park, H. G. Mismatch DNASpecific Enzymatic Cleavage Employed in a New Method for the Electrochemical Detection of Genetic Mutations. Chem. Commun. 2009, 45 (28), 4230-4232.

264. Stewart, M. E.; Anderton, C. R.; Thompson, L. B.; Maria, J.; Gray, S. K.; Rogers, J. A.; Nuzzo, R. G. Nanostructured Plasmonic Sensors. Chem. Rev. 2008, 108 (2), 494-521.

265. Mout, R.; Moyano, D. F.; Rana, S.; Rotello, V. M. Surface Functionalization of Nanoparticles for Nanomedicine. Chem. Soc. Rev. 2012, 41 (7), 2539-2544. 
266. Grabar, K. C.; Freeman, R. G.; Hommer, M. B.; Natan, M. J. Preparation and Characterization of Au Colloid Monolayers. Anal. Chem. 1995, 67 (4), 735-743.

267. Hurst, S. J.; Lytton-Jean, A. K.; Mirkin, C. A. Maximizing DNA Loading on a Range of Gold Nanoparticle Sizes. Anal. Chem. 2006, 78 (24), 8313-8318.

268.Liu, J. W.; Lu, Y. Colorimetric $\mathrm{Cu}^{2+}$ Detection with a Ligation DNAzyme and Nanoparticles. Chem. Commun. 2007, 43 (46), 4872-4874.

269. Khlebtsov, N. G. Determination of Size and Concentration of Gold Nanoparticles from Extinction Spectra. Anal. Chem. 2008, 80 (17), 6620-6625.

270. Shida, T.; Noda, M.; Sekiguchi, J. Cleavage of Single- and Double-Stranded DNAs Containing an Abasic Residue by Escherichia Coli Exonuclease III (AP endonuclease VI). Nucleic Acids Res. 1996, 24 (22), 4572-4576.

271. Henikoff, S. Unidirectional Digestion with Exonuclease III Creates Targeted Breakpoints for DNA Sequencing. Gene 1984, 28 (3), 351-359.

272. Takeuchi, M.; Lillis, R.; Demple, B.; Takeshita, M. Interactions of Escherichia Coli Endonuclease IV and Exonuclease III with Abasic Sites in DNA. J. Biol. Chem. 1994, 269 (34), 21907-21914.

273. Zhang, L.K.; Rempel, D.; Gross, M. L. Matrix-Assisted Laser Desorption/Ionization Mass Spectrometry for Locating Abasic Sites and Determining the Rates of Enzymatic Hydrolysis of Model Oligodeoxynucleotides. Anal. Chem. 2001, 73 (14), 3263-3273.

274. Wilson, D. M. Ape1 Abasic Endonuclease Activity is Regulated by Magnesium and Potassium Concentrations and is Robust on Alternative DNA Structures. J. Mol. Biol. 2005, 345 (5), 1003-1014.

275. Sági, J.; Hang, B.; Singer, B. Sequence-Dependent Repair of Synthetic AP Sites in 15-mer and 35-mer Oligonucleotides: Role of Thermodynamic Stability Imposed by Neighbor Bases. Chem. Res. Toxicol. 1999, 12 (10), 917-923.

276. Bellamy, S. R.; Kovacheva, Y. S.; Zulkipli, I. H.; Halford, S. E. Differences Between $\mathrm{Ca}^{2+}$ and $\mathrm{Mg}^{2+}$ in DNA Binding and Release by the SfiI Restriction Endonuclease: Implications for DNA Looping. Nucleic Acids Res. 2009, 37 (16), 5443-5453.

277. Erzberger, J. P.; Wilson D. M. The Role of $\mathrm{Mg}^{2+}$ and Specific Amino Acid Residues in the Catalytic Reaction of the Major Human Abasic Endonuclease: New Insights from EDTAResistant Incision of Acyclic Abasic Site Analogs and Site-Directed Mutagenesis. J. Mol. Biol. 1999, 290 (2), 447-457.

278. Pei, H.; Lu, N.; Wen, Y. L.; Song, S. P.; Liu, Y.; Yan, H.; Fan, C. H. A DNA NanostructureBased Biomolecular Probe Carrier Platform for Electrochemical Biosensing. Adv. Mater. 2010, 22 (42), 4754-4758.

279. Fish, D. J.; Horne, M. T.; Brewood, G. P.; Goodarzi, J. P.; Alemayehu, S.; Bhandiwad, A.; Searles, R. P.; Benight, A. S. DNA Multiplex Hybridization on Microarrays and 
Thermodynamic Stability in Solution: a Direct Comparison. Nucleic Acids Res. 2007, 35 (21), 7197-7208.

280. Kow, Y. W. Mechanism of Action of Escherichia coli Exonuclease III. Biochemistry 1989, $28(8), 3280-3287$.

281. Dubertret, B.; Calame, M.; Libchaber, A. J. Single-Mismatch Detection Using GoldQuenched Fluorescent Oligonucleotides. Nat. Biotechnol. 2001, 19 (4), 365-370.

282. Heid, C. A.; Stevens, J.; Livak, K. J.; Williams, P. M. Real Time Quantitative PCR. Genome Res. 1996, 6 (10), 986-994.

283. Bock, L. C.; Griffin, L. C.; Latham, J. A.; Vermaas, E. H.; Toole, J. J. Selection of SingleStranded DNA Molecules that Bind and Inhibit Human Thrombin. Nature 1992, 355 (6360), 564-566.

284.Demers, L. M.; Mirkin, C. A.; Mucic, R. C.; Reynolds, R. A.; Letsinger, R. L.; Elghanian, R.; Viswanadham, G. A Fluorescence-Based Method for Determining the Surface Coverage and Hybridization Efficiency of Thiol-Capped Oligonucleotides Bound to Gold Thin Films and Nanoparticles. Anal. Chem. 2000, 72 (22), 5535-5541.

285. Richardson, C. C.; Kornberg, A. A Deoxyribonucleic Acid Phosphatase-Exonuclease from Escherichia coli : I. Purification of the Enzyme and Characterization of the Phosphatase Activity. J. Biol. Chem. 1964, 239 (1), 242-250.

286. Mol, C. D.; Kuo, C. F.; Thayer, M. M.; Cunningham, R. P.; Tainer, J. A. Structure and Function of the Multifunctional DNA-Repair Enzyme Exonuclease III. Nature 1995, 374 (6520), 381-386

287. Roncancio, D.; Yu, H. X; Xu, X. W; Wu, S.; Liu, R.; Debord, J.; Lou, X. H; Xiao, Y. A Label-Free Aptamer-Fluorophore Assembly for Rapid and Specific Detection of Cocaine in Biofluids. Anal. Chem. 2014, 86 (22), 11100-11106.

288. Kent, A. D.; Spiropulos, N. G.; Heemstra, J. M. General Approach for Engineering SmallMolecule-Binding DNA Split Aptamers. Anal. Chem. 2013, 85 (20), 9916-9923.

289. Tomb, J. F.; Barcak, G. J. Regulating the 3'-5' Activity of Exonuclease III by Varying the Sodium Chloride Concentration. BioTechniques 1989, 7 (9), 932-933. 
VITA

\section{PINGPING LIANG}

EDUCATION

Born, Xi'an, Shaanxi, China

$2003-2007$

B.S., Chemistry

Shaanxi Normal University, Xi'an, Shaanxi, China

$2011-2016$

Doctoral Candidate

Florida International University, Miami, Florida, USA

PUBLICATIONS and PRESENTATIONS

1. Liang P.P., Fan Y.S., Rodriguez, L., Xiao Y. "DTT-regulated space for sensitive colorimetric detection of effective formation of target/aptamer complex on split aptamermodified nanoparticle surfaces.” 2016, Submitted.

2. Yu H.X., Liang P.P., Xu X.W., Guntupalli B., Lou X.H., Xiao Y. "Universally applicable assay for rapidly and accurately quantifying DNA surface coverage on diverse particles." 2016, Submitted.

3. Guntupalli, B., Liang, P.P., Lee, J.H., Yang Y.H., Yu H.X., Canoura J., He J., Li W.Z., Weizmann Y., Xiao Y. "Ambient filtration method to rapidly prepare highly conductive, paper-based porous gold films for electrochemical biosensing." ACS Appl. Mater. Interfaces, 2015, 7, 27049-27058. (IF 6.723)

4. Liang P.P., Yu H.X. Guntupalli B., Xiao Y. "Paper-based device for rapid visualization of NADH based on dissolution of gold nanoparticles." ACS Appl. Mater. Interfaces. 2015, 7, 15023-15030. (IF 6.723)

5. Wu S., Liang P.P., Yu H.X., Xu X.W., Liu Y., Lou X.H., Xiao Y. “Amplified single base-pair mismatch detection via aggregation of exonuclease-sheared gold nanoparticles." Anal. Chem. 2014, 86, 3461-3467. (IF 5.636)

6. Liang P.P., Yu H.X., Guntupalli B., Xiao Y. "Paper-based device for rapid visualization of NADH based on dissolution of gold nanoparticles." 2016 Conference of Undergraduate Research at FIU (CURFIU), Miami, FL, 03/30 - 03/31. (Oral Presentation)

7. Liang P.P., Yu H.X., Guntupalli B., Xiao Y. "Paper-based device for rapid visualization of NADH based on dissolution of gold nanoparticles." 2016 FIU's annual Scholarly 
Forum during Graduate Student Appreciation Week (GSAW), Florida International University, Miami, FL, 03/28 - 03/29. (Poster Presentation)

8. Liang P.P., Yu H.X., Xiao Y. "Paper-based device for rapid visualization of NADH based on dissolution of gold nanoparticles." 2015 Fall MRS meeting, Boston, Massachusetts, United States, 11/29 - 12/04. (Poster Presentation)

9. Guntupalli B., Liang P.P., Yang Y.H., He J., Li W.Z., Xiao Y. "Rapid, room-temperature preparation of highly conductive, paper based porous gold film." 2015 Fall MRS meeting, Boston, Massachusetts, United States, 11/29 - 12/04. (Oral Presentation)

10. Wu S., Liang P.P., Yu H.X., Xu X.W., Liu Y., Lou X.H., Xiao Y. “Amplified single base-pair mismatch detection via aggregation of exonuclease-sheared gold nanoparticles.” 2014 Fall MRS meeting, Boston, Massachusetts, United States, 11/30 12/05. (Poster Presentation)

11. Guntupalli B., Liang, P.P., Yang Y.H., He J., Li W.Z., Xiao Y. “Ambient filtration method to prepare paper-based electroactive nonoporous gold thin film for detection of dopamine and serotonin.” 2013-89th Florida Annual Meeting and Exposition (FAME), Tampa, FL, United States, 05/09 - 05/11. (Oral presentation)

12. Dai J.Y., Paudyal J., Guntupalli B., Liang P.P., Yang Y.H., Tiwari P., Li W.H., He J., Wang, X.T., Xiao Y. "Paper based single-wall carbon nanotube thin film for catalytic detection for nicotineamide adenine dinucleotilde in human blood." 2013-89th Florida Annual Meeting and Exposition (FAME), Tampa, FL, United States, 05/09 - 05/11. (Oral presentation) 